Leandro Vanalli

\title{
O MEC E O MEF APLICADOS À ANÁLISE DE PROBLEMAS VISCOPLÁSTICOS EM MEIOS ANISOTRÓPICOS E COMPOSTOS
}

Tese apresentada à Escola de Engenharia de São Carlos da Universidade de São Paulo, como parte dos requisitos para a obtenção do Título de Doutor em Engenharia de Estruturas.

ORIENTADOR: Prof. Ass. Humberto Breves Coda 
À Simone e Pedro, Osvaldo e Fátima. 


\section{AGRADECIMENTOS}

Ao meu Senhor e Salvador Jesus Cristo.

Ao Prof. Humberto Breves Coda, pela eficiente orientação e confiança depositada, mas principalmente pela amizade que surgiu durante a realização desta pesquisa.

Aos grandes amigos Rodrigo Ribeiro Paccola, Everaldo Bonaldo e Wilson Wesley Wutzow.

Aos amigos do Departamento: Daniel, Patrick, Greco, Oscar, Josafá, Cilmar, Botta, Adilson, Carrazedo, Rodrigo e Andrei.

Aos professores e funcionários do Departamento de Engenharia de Estruturas que contribuíram de alguma forma para a realização deste trabalho.

À minha esposa Simone, pelo amor, paciência, orações e incentivo para que eu pudesse continuar nos momentos difíceis.

Aos meus familiares que sempre me apoiaram em oração.

Ao pastor Nelson Nunes da Silva e sua amável família, pelas orações e incentivo.

À Fundação de Amparo à Pesquisa do Estado de São Paulo - FAPESP, pelo financiamento da pesquisa. 
"E chamou Abraão o nome daquele lugar: $O$ SENHOR PROVERÁ; donde se diz até ao dia de hoje: No monte do SENHOR se proverá”

Gênesis 22:14 


\section{RESUMO}

VANALLI, L. (2004). O MEC e o MEF aplicados à análise de problemas viscoplásticos em meios anisotrópicos e compostos. Tese (Doutorado), 194p - Escola de Engenharia de São Carlos, Universidade de São Paulo, São Carlos. 2004.

O objetivo do presente trabalho é o desenvolvimento de formulações e de códigos computacionais que possibilitem a análise bidimensional estática de meios contínuos anisotrópicos viscoplásticos reforçados ou não por fibras. Especificamente, as análises numéricas envolvem aplicações dos Métodos dos Elementos de Contorno (MEC) e dos Elementos Finitos (MEF), comparando-se os resultados obtidos com respostas analíticas e experimentais, disponíveis na literatura, buscando-se assim, subsídios teóricos que permitam o entendimento de problemas mais gerais envolvendo meios anisotrópicos. Para tanto são empregados elementos finitos triangulares com aproximações cúbica e quadrática para os deslocamentos na modelagem dos domínios. Na consideração do reforço com fibras, elementos finitos de barras simples são empregados. A formulação desenvolvida proporciona também a consideração de distribuição randômica das fibras imersas no meio sem qualquer aumento dos graus de liberdade do problema analisado, diferindo-se assim, das formulações conhecidas até o momento. Com o MEC, a análise de plasticidade e viscoplasticidade em meios com anisotropia geral é feita de maneira original no trabalho, destacando-se a consideração de lei de fluxo plástico nãoassociativa e o tratamento de viscosidade apenas com integrais de contorno, sem a utilização de aproximações de domínio. Uma quantidade significativa de exemplos é apresentada, possibilitando a verificação da eficiência das formulações e dos códigos desenvolvidos.

Palavras-chave: Método dos Elementos de Contorno; Método dos Elementos Finitos; anisotropia; materiais compósitos; não-linearidade física; fibras curtas. 


\section{ABSTRACT}

VANALLI, L. (2004). The BEM and FEM applied for analysis of viscoplastics problems in the anisotropic and composites medias. Ph.D Thesis, 194p - Escola de Engenharia de São Carlos, Universidade de São Paulo, São Carlos. 2004.

The objective of the present work is the development of formulations and computational codes that enable the static bidimensional analysis of the viscoplastic anisotropic medias reinforced, or not, by fibers. Specifically, the numerical analysis involve applications of the Boundary Elements Method (BEM) and Finite Elements (FEM), comparing the results obtained with analytical and experimental solutions available in the literature, allowing the understanding of general problems in anisotropic media. Two-dimensional finite elements with cubic and quadrate approximations for the displacements are used to model domains. Reinforcements are modeled by truss finite elements. The developed formulation provides the consideration of random distribution of the fibers, without any additional degree of freedom of the problem. With the BEM, the plasticity and viscoplasticity analysis in general anisotropic medias is originally developed in the present work, emphasizing the consideration of non-associative plastic flow and the treatment of viscosity just with boundary integrals, without domain approximation. Various examples are shown in order to verify the efficiency of the proposed formulation and developed computational codes.

Keywords: Boundary Elements Method; Finite Elements Method; anisotropy; composite materials; non-linearity physics; short fibers. 


\section{SUMÁRIO}

RESUMO

$\begin{array}{ll}\text { ABSTRACT } & 7\end{array}$

1 INTRODUÇÃO 12

$\begin{array}{ll}\text { 1.1 GENERALIDADES } & 12\end{array}$

$\begin{array}{lr}1.2 & 14\end{array}$

$\begin{array}{ll}\text { 1.3 RESUMOS DOS CAPÍTULOS } & 15\end{array}$

2 REVISÃO DA LITERATURA 17

2.1 O MEF APLICADO A PROBLEMAS ANISOTRÓPICOS 18

2.2 O MEC APLICADO A PROBLEMAS ANISOTRÓPICOS 21

3 MEIOS ANISOTRÓPICOS - CONSIDERAÇÕES GERAIS E CRITÉRIOS DE RESISTÊNCIA 28

$\begin{array}{lc}\text { 3.1 GENERALIDADES } & 28\end{array}$

3.2 LEIS CONSTITUTIVAS PARA MATERIAIS ELÁSTICOS ANISOTRÓPICOS

3.2.1 Representação das Leis Constitutivas 31

3.2.2 Classificação dos Materiais segundo o Número de Planos de 35 Simetria Elástica

3.2.2.1 Material com Simetria Elástica em um Plano 36

3.2.2.2 Material com Simetria Elástica em Três Planos (Material 38 Ortotrópico)

3.2.2.3 Material Transversalmente Isotrópico (Um Plano de Isotropia)

3.2.2.4 Material Isotrópico

3.3 DESCRIÇÃO DA SOLUÇÃO FUNDAMENTAL ANISOTRÓPICA UTILIZADA

3.4 CONSIDERAÇÕES GERAIS SOBRE RESISTÊNCIA DOS MATERIAIS ANISOTRÓPICOS 
3.4.1 Critérios de ruptura para materiais anisotrópicos e compostos 4 FORMULAÇÃO DO MEF PARA O PROBLEMA VISCOPLÁSTICO ANISOTRÓPICO

4.1 FORMULAÇÃO INTEGRAL PARA A ABORDAGEM DO PROBLEMA

4.2 TRATAMENTO ALGÉBRICO DA REPRESENTAÇÃO INTEGRAL

5 FORMULAÇÃO DO MEC PARA O PROBLEMA VISCOPLÁSTICO ANISOTRÓPICO

5.1 FORMULAÇÃO INTEGRAL PARA A ABORDAGEM DO PROBLEMA

5.1.1 Representação Integral para Tensões em Pontos Internos

5.1.2 Determinação das Tensões Totais no Contorno

5.2 TRATAMENTO ALGÉBRICO PARA AS REPRESENTAÇÕES INTEGRAIS

5.2.1 Integração temporal numérica

6 ALGORITMO PARA ATUALIZAÇÃO DAS TENSÕES CRITÉRIO DE TSAI-WU

6.1 GENERALIDADES

6.2 CONSIDERAÇÕES GERAIS SOBRE PLASTICIDADE

6.3 ASPECTOS INCREMENTAIS DO ALGORITMO

6.4 ALGORITMO TSAI \& WU (1971) NÃO ASSOCIATIVO

7 APLICAÇÕES - ANÁLISE ELÁSTICA LINEAR

7.1 EXEMPLO 1 - VIGA ENGASTADA

7.2 EXEMPLO 2 - VIGA SIMPLESMENTE APOIADA COM UM FURO NO DOMÍNIO

7.3 EXEMPLO 3 - DETERMINAÇÃO DE FATORES DE INTENSIDADE DE TENSÃO KI 
8 APLICAÇÕES - ANÁLISE VISCOELÁSTICA 106

8.1 EXEMPLO 1 - CHAPA QUASE-ISOTRÓPICA 106

8.2 EXEMPLO 2 - CHAPA COM ANISOTROPIA GERAL 109

9 APLICAÇÕES - ANÁLISE VISCOPLÁSTICA 112

9.1 EXEMPLO 1 - CHAPA QUASE-ISOTRÓPICA 113

9.2 EXEMPLO 2 - CHAPA ORTOTRÓPICA 120

9.3 EXEMPLO 3 - CHAPA COM ANISOTROPIA GERAL 123

10 MEIOS REFORÇADOS COM FIBRAS - ANÁLISE

UTILIZANDO-SE O MEF

10.1 GENERALIDADES

131

10.2 SISTEMAS COM FIBRAS

132

10.3 FORMULAÇÃO DO MEF PARA A CONSIDERAÇÃO DO

REFORÇO NUMA POSIÇÃO QUALQUER DO DOMÍNIO

130

10.4 APLICAÇÕES DO CÓDIGO DESENVOLVIDO

135

139

10.4.1 Análise de Chapas Reforçadas em Regime Elástico-Linear $\quad 140$

10.4.1.1 Chapa ortotrópica - fibras dispostas unidirecionalmente $\quad 140$

10.4.1.2 Chapa ortotrópica - fibras dispostas em duas direções 141

ortogonais

10.4.1.3 Chapa com anisotropia geral - fibras dispostas em duas direções ortogonais e não coincidentes com as direções globais do problema

10.4.2 Viga de Concreto Armado

10.4.3 Viga de Concreto Reforçado com Fibras de Aço - Ensaios de Furlan (1995)

11 CONCLUSÃO

11.1 CONSIDERAÇÕES CONCLUSIVAS GERAIS

11.2 CONCLUSÕES RELACIONADAS COM AS APLICAÇÕES 
REFERÊNCIAS BIBLIOGRÁFICAS

ANEXO 1 - DETALHAMENTO DA SOLUÇÃO FUNDAMENTAL DE CRUSE \& SWEDLOW (1971)

ANEXO 2 - ESTRATÉGIA NUMÉRICA PARA O CÁLCULO DO TERMO LIVRE - ANÁLISE DE PLASTICIDADE

ANEXO 3 - DETERMINAÇÃO DAS COORDENADAS ADMENSIONAIS DE UM PONTO CARTESIANO 


\section{INTRODUÇÃo}

\subsection{GENERALIDADES}

Desde os tempos mais remotos da sociedade humana, o homem tem se dedicado ao entendimento dos fenômenos físicos da natureza, muitas vezes motivado pela necessidade de sobrevivência ou, até mesmo, movido pelo desejo de se superar e modificar para melhor a vida de sua sociedade.

Assim, nos primeiros séculos, as técnicas de Engenharia eram baseadas apenas na própria experimentação e simples observação do homem, sem nenhuma base matemática como, por exemplo, pode-se citar o relato bíblico do homem prudente que edificou sua casa sobre a rocha, “...e caiu a chuva, transbordaram os rios, sopraram os ventos e deram com ímpeto contra aquela casa, que não caiu...” (Mateus, 7:24).

Porém, no decorrer dos anos, por meio da ousadia e inspiração de poucos, muitas vezes contrária à razão de muitos, modelos matemáticos para a interpretação das leis mecânicas da natureza foram sendo criados, permitindo-se assim, para aqueles que se dedicavam à Engenharia, o estudo do comportamento estrutural fundamentado em modelos mecânicos e matemáticos.

No século passado, graças ao grau de desenvolvimento da Matemática e da Física a partir do século dezessete, foi possível o surgimento dos computadores, e assim, outra grande área de modelagem pôde ser desenvolvida, a modelagem via computador, também conhecida como modelagem numérica, que cada vez mais ganha espaço e atualmente é imprescindível nas mais diferentes áreas do conhecimento humano devido sua eficiência e versatilidade. Para tornar essa modelagem possível foram desenvolvidos os métodos numéricos e, a partir desses, a elaboração de códigos computacionais para a análise dos problemas de Engenharia. 
Os métodos numéricos de domínio, como o Método dos Elementos Finitos (MEF) e o Método das Diferenças Finitas (MDF), desenvolvidos já há algum tempo, foram sempre os mais utilizados como ferramentas básicas na elaboração dos sistemas computacionais destinados à análise de problemas de Engenharia, em particular, aos relacionados à Engenharia Estrutural.

O surgimento do Método dos Elementos de Contorno (MEC), como uma alternativa para a resolução de quase todos os problemas da Engenharia é, sem dúvida, um avanço científico muito significativo nessa área do conhecimento (VENTURINI, 1988). Uma das principais vantagens desse método, quando comparado com o MEF, é a redução do número de variáveis do problema, pois enquanto nos métodos usuais o domínio a ser tratado precisa ser dividido em vários subdomínios, no MEC, tratando-se de análises lineares, apenas o contorno do mesmo precisa ser discretizado.

Dessa forma, inserido no contexto apresentado para os métodos numéricos mais difundidos no campo da Engenharia, o estudo da anisotropia se fundamenta devido sua importância no âmbito da Engenharia Estrutural, pois, diversos materiais de uso corrente se comportam de forma anisotrópica, citando-se, como exemplos, materiais reforçados com fibras (concretos e materiais poliméricos), alvenaria estrutural e a madeira.

Devido a importância do tema para a Engenharia, desde a década de 50, muitos pesquisadores têm se preocupado com o estudo de meios anisotrópicos, principalmente abordando aspectos teóricos visando a obtenção de soluções analíticas aproximadas para diversos problemas (LEKHNITISKII, 1963). No entanto, devido a maior complexidade matemática inerente à anisotropia, essas soluções são sempre limitadas às estruturas mais simples, com condições de contorno simplificadas.

Nesse sentido, o presente trabalho destina-se à análise de problemas anisotrópicos, através do MEC e do MEF, buscando-se o desenvolvimento de formulações e de códigos computacionais que permitam interpretar mais realísticamente o comportamento das estruturas. Assim, com a modelagem numérica, uma gama mais significativa de problemas pode ser abordada, dando-se suporte teórico e numérico para futuras aplicações tecnológicas. 


\subsection{OBJETIVOS}

O objetivo principal do trabalho é o desenvolvimento de formulações e de dois códigos computacionais, um baseado no MEC e outro no MEF, que permitam a análise bidimensional quase estática de meios contínuos anisotrópicos viscoplásticos, reforçados ou não por fibras.

Para tanto, no decorrer do trabalho, buscou-se um aprofundamento teórico nos assuntos sobre os quais a pesquisa está fundamentada, fazendo-se um levantamento bibliográfico sobre soluções fundamentais para meios não isotrópicos e também sobre não linearidade física e leis de fluxo plástico para esses meios.

A implementação de dois programas similares é justificada pela possibilidade de se obter comparações, em virtude da escassez de trabalhos destinados à análise de meios anisotrópicos não lineares, dos resultados obtidos com os dois métodos. Salientase, que no decorrer do desenvolvimento do trabalho, se optou pela introdução de modelagem das fibras apenas no programa de elementos finitos, pela razão dessa modelagem, randômica e sem aumento dos graus de liberdade do problema, constituir contribuição original à área de Estruturas.

Destaca-se também como contribuição científica inovadora da presente pesquisa, principalmente para a área de modelagem numérica com o MEC, o tratamento de plasticidade em meios com anisotropia geral. Tal desenvolvimento exigiu adoção de procedimentos originais no manuseio da solução fundamental empregada, dependente de variáveis complexas, como por exemplo, a determinação numérica do termo livre, que para a solução fundamental de Kelvin possui uma expressão analítica simples bem conhecida (BUI, 1978).

Ainda em relação ao MEC, na abordagem dos problemas com viscosidade, diferentemente de procedimentos usuais, não são empregadas aproximações de domínio e a viscosidade é representada apenas por integrais de contorno. Para o tratamento de plasticidade, células triangulares com aproximação quadrática são empregadas.

Os dois códigos computacionais foram desenvolvidos integralmente nesta pesquisa, preocupando-se sempre em documentá-los de maneira detalhada para que seja possível a utilização deles em trabalhos futuros. 


\subsection{RESUMOS DOS CAPÍTULOS}

No capítulo 1, apresenta-se uma introdução ao trabalho, seus objetivos, os aspectos inovadores alcançados, bem como, um breve comentário sobre a metodologia empregada.

No capítulo 2, descrevem-se resumidamente as principais referências bibliográficas pesquisadas no trabalho, que serviram de base teórica para o mesmo.

No capítulo 3 são apresentadas considerações gerais sobre meios anisotrópicos, suas relações constitutivas, destacando-se aspectos básicos que as diferenciam das relações de outros meios, e também, a solução fundamental empregada é brevemente descrita. Aborda-se ainda, alguns critérios de resistência específicos para materiais nãoisotrópicos no intuito de se definir qual critério é o mais apropriado para as análises, em meios com anisotropia geral, propostas nesta pesquisa.

No capítulo 4, descreve-se a formulação do MEF para o problema viscoplástico anisotrópico, apresentando-se os equacionamentos integral e algébrico, os elementos finitos utilizados, bem como a maneira como a viscosidade será tratada.

Semelhantemente ao que foi desenvolvido para o MEF no capítulo 4, faz-se com o MEC no capítulo 5. A formulação para os problemas com viscosidade é obtida considerando-se o amortecimento viscoso apenas no contorno do corpo, com integrais de contorno, sem a utilização de células de domínio. Para o tratamento da plasticidade, são utilizadas células triangulares com aproximação quadrática.

No capítulo 6, apresenta-se uma breve introdução à teoria da plasticidade, apresentando-se uma abordagem numérica do problema elastoplástico e definindo-se os algoritmos para a solução do problema não linear. Os algoritmos são do tipo implícito com leis de fluxo não-associativas. Obtém-se uma expressão fechada para o multiplicador plástico, evitando-se assim, a utilização de processos iterativos para o cálculo do mesmo.

No capítulo 7, são apresentadas aplicações das formulações, MEC e MEF, elastostáticas empregadas. Os exemplos elásticos lineares abordados permitem observar, com mais clareza, as particularidades inerentes ao comportamento mecânico dos meios anisotrópicos. Uma importante constatação sobre aproximações, pelo MEF, para o problema envolvendo anisotropia geral é alcançada. 
No capítulo 8 são apresentados exemplos de aplicação, para análises viscoelásticas, dos dois códigos desenvolvidos. Os resultados obtidos mostram a eficiência da formulação viscosa empregada.

Problemas viscoplásticos são abordados no capítulo 9, apresentando-se, especialmente, resultados do programa de elementos de contorno para meios com anisotropia geral, os quais, são contribuições científicas originais da presente pesquisa.

O capítulo 10 traz uma formulação alternativa para o tratamento de meios anisotrópicos reforçados com fibras através do MEF. São abordados aspectos gerais desses meios, bem como a formulação desenvolvida que permite a consideração de distribuição aleatória das fibras no domínio do problema, sem qualquer reordenação de malha e nem aumento dos graus de liberdade da análise. Os resultados, comparados com soluções numéricas e experimentais obtidas na literatura, mostram a eficiência da técnica desenvolvida.

No último capítulo são apresentadas as conclusões da presente tese de doutoramento e sugestões para a continuação desta linha pesquisa. 


\section{REVISÃO DA LITERATURA}

Neste capítulo, de forma objetiva, apresentam-se importantes referências da literatura sobre os assuntos abordados nesta pesquisa. Com isso, citam-se alguns trabalhos teóricos sobre aplicação da teoria da Elasticidade no estudo de meios não isotrópicos, e também, sobre a análise numérica desses meios, especificamente, a utilização dos Métodos dos Elementos Finitos (MEF) e dos Elementos de Contorno (MEC).

Devido ao aumento das aplicações industriais dos materiais compósitos, a teoria da Elasticidade aplicada ao estudo de meios anisotrópicos tem sido motivo de atenções a partir da década de 60, destacando-se, a pessoa de Lekhnitskii, maior referência teórica na área de Engenharia sobre o assunto, cujos trabalhos principais (LEKHNITSKII, 1963; LEKHNITSKII et al., 1968) abordam muitos aspectos matemáticos da teoria da Elasticidade anisotrópica, bem como, apresentam soluções analíticas aproximadas para diversos problemas com anisotropia.

Um outro trabalho teórico importante, na linha da teoria da Elasticidade, é o de TING (1996), onde são apresentados também muitos aspectos matemáticos e soluções analíticas para diversos problemas em meios anisotrópicos, incluindo-se problemas mais complexos dos abordados por LEKHNITSKII (1963), como por exemplo, inclusões elípticas e mecânica da fratura.

Outros estudos teóricos sobre meios anisotrópicos, com diversas aplicações, podem ainda ser mencionados. GREEN \& ZERNA (1954) apresentaram um estudo sobre a distribuição de tensões numa chapa ortotrópica tracionada com um orifício interno. Essas distribuições são comparadas com um material isotrópico e pode-se 
observar os diferentes comportamentos mecânicos da chapa, dependendo-se da direção de aplicação das tensões.

SILVERMAN (1964), estudando tensões e deformações em chapas ortotrópicas submetidas a cargas normais e tangenciais, desenvolveu um método de solução analítico aproximado, através da utilização de funções de tensão de Airy polinomiais de vários graus, empregando seu método em vários exemplos.

HASHIN (1967), apresentou um método analítico de resolução de problemas planos envolvendo chapas anisotrópicas, submetidas a carregamentos polinomiais, 0 qual permite a construção de funções de tensão polinomiais, semelhantes às funções de Airy, que são soluções de problemas planos anisotrópicos. São apresentadas aplicações em chapas com anisotropia geral. NOACK \& ROTH (1976) apresentam uma análise matemática da teoria da Elasticidade para materiais ortotrópicos, considerando-se a anisotropia retilinear e a cilíndrica. Em seguida são feitas algumas aplicações da teoria para estruturas curvas em madeira laminada.

Sobre a anisotropia da madeira, MASCIA (1991) fez um estudo abrangente, considerando várias espécies brasileiras, apresentando resultados de ensaios de compressão e flexão, onde é possível verificar o alto grau de anisotropia presente nesse material. Utilizando-se os dados de ensaios de MASCIA (1991), VANALLI (2001) fez um estudo teórico sobre aplicações das funções de tensão no estudo de meios anisotrópicos, utilizando-se também, o método de HASHIN (1967) para a determinação de soluções para alguns problemas planos com anisotropia geral.

Tendo-se apresentado algumas referências destinadas à análise teórica de problemas com anisotropia, é importante comentar que o número de trabalhos que abordam este tema não é muito grande, e como já comentado, a maioria deles têm como base os estudos teóricos de Lekhnitskii. No item seguinte, apresenta-se um breve comentário sobre o MEF, bem como, uma revisão bibliográfica sobre sua aplicação em problemas com anisotropia.

\subsection{O MEF APLICADO A PROBLEMAS ANISOTRÓPICOS}

A complexidade do modelo matemático que representa o comportamento de muitos problemas de Engenharia levou ao desenvolvimento de métodos aproximados para sua solução, podendo-se destacar o MEF. Da forma como hoje é conhecida, a 
formulação do MEF foi estabelecida com a publicação do trabalho de TURNER et al. (1956). Clough apud ASSAN (1999), foi o autor do nome "MEF" e num artigo publicado em 1980 descreve em detalhes sua participação no desenvolvimento do método.

De uma forma simplificada, pode-se dizer que o MEF consiste em transformar o sólido contínuo em uma associação de elementos discretos (elementos finitos), como mostrado na Figura 2.1. Escrevem-se equações de compatibilidade e de equilíbrio entre os elementos e também, admitem-se funções aproximadoras contínuas, formadas por variáveis referidas aos nós dos elementos e por funções denominadas funções de forma, que representam, por exemplo, o campo de deslocamentos no domínio do elemento. A partir da resolução de um sistema de equações algébricas lineares formado por essas funções contínuas, obtém-se os deslocamentos referidos aos nós de cada elemento finito.

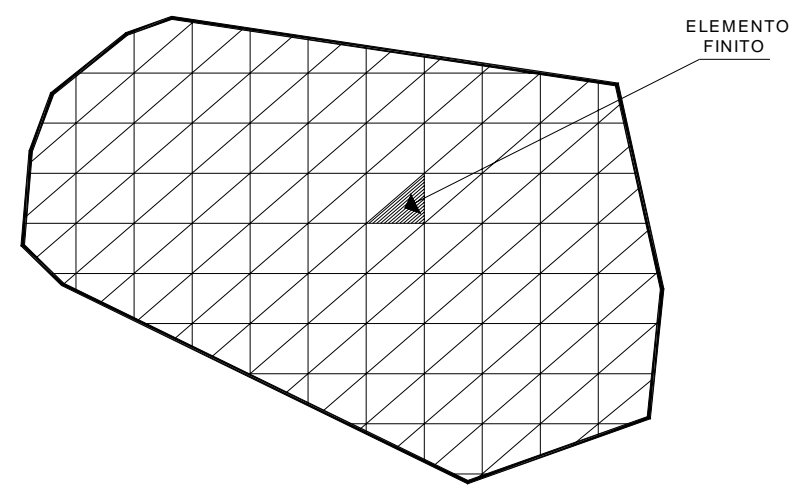

FIGURA 2.1- Domínio com uma malha de elementos finitos.

Com os deslocamentos, pode-se então obter o estado de deformações que, associado às relações constitutivas do material, permitem definir o estado de tensão em cada elemento finito.

Atualmente, utilizando-se do MEF, existem muitos códigos computacionais desenvolvidos com a finalidade de se analisar diversos problemas correntes na Engenharia. Tratando-se particularmente de problemas envolvendo sólidos anisotrópicos, são citados alguns trabalhos com diferentes abordagens.

Um dos primeiros trabalhos foi o de WILSON (1965), onde o MEF foi aplicado para a solução de sólidos axi-simétricos, onde a anisotropia, bem como, a não simetria das condições de carregamento é incluída. AL DABBAGH et al. (1972) desenvolveram um código para a análise tri-dimensional de sólidos com anisotropia 
geral, utilizando elementos em forma de paralelepípedo, com aplicações específicas à madeira.

FIGUEIRAS (1983) aplicou o MEF na análise não linear de placas e cascas anisotrópicas. Duas diferentes formulações de elementos finitos são apresentadas e ênfase especial é dada para análise de estruturas de concreto, compostas e laminares.

GIAVOTTO et al. (1983) aplicaram o MEF, com elementos finitos isoparamétricos, na análise linear de sólidos anisotrópicos planos, voltando-se principalmente para o estudo de hélices de helicópteros e geradores de usinas eólicas, constituídos de materiais reforçados por fibras modelados por técnica de homogeneização.

Na análise não linear física de estruturas anisotrópicas com o MEF, BRÜNIG (1995) estudou o comportamento elasto-plástico (não associativo) dessas estruturas utilizando-se de uma aproximação quadrática para o critério de ruptura de TSAI \& WU (1971). São analisados alguns exemplos onde o efeito da anisotropia sobre o escoamento é discutido.

FERREIRA et al. (1999) analisaram com elementos finitos de casca, o comportamento elasto-plástico de estruturas laminadas (sandwich e compósitas) tendose em conta três abordagens cinemáticas e uma teoria associativa de fluxo plástico. São apresentados vários exemplos evidenciando o comportamento particular dessas estruturas.

HOLMBERG et al. (1999), fizeram um estudo sobre o comportamento não linear físico da madeira e de alguns materiais fibrosos. Caracterizaram, e modelaram com o MEF, as propriedades mecânicas (de rigidez e de fluência) de algumas espécies, considerando variações de densidade e utilizando um procedimento de homogeneização. Resultados experimentais e numéricos são comparados.

$\mathrm{Na}$ análise linear de vigas compósitas espessas, e de placas, ambas consideradas anisotrópicas, YILDIZ \& SARIKANAT (2001) aplicaram o MEF para a determinação das propriedades elásticas dessas estruturas, considerando-as laminadas. Tratando-se ainda da análise linear de vigas laminadas, pode-se citar o trabalho de RAVEENDRANATH et al. (2000), que utilizaram o MEF, com elementos finitos curvos de dois nós, com três graus de liberdade por nó, considerando ainda, campos de deslocamentos cúbicos, para o estudo dessas vigas submetidas a diferentes condições de carregamento. Outros trabalhos podem ainda ser citados, como por exemplo, o de POPESCU \& HODGES (2000), na análise dos efeitos do cisalhamento em vigas 
anisotrópicas com seções transversais de diferentes formas, e de QIN \& LIBRESCU (2002) na modelagem de vigas anisotrópicas com seções transversais do tipo caixão.

É importante comentar, após a apresentação de alguns trabalhos, que apesar da grande difusão do MEF, a análise numérica de meios com anisotropia geral não é um tema muito explorado e, devido a esse fato, até o presente momento, não se encontrou na literatura algum trabalho destinado à análise viscoplástica desses meios, justificandose assim, a ausência de referências sobre esse tema.

A seguir, apresenta-se uma descrição geral sobre o MEC e também, algumas aplicações em problemas anisotrópicos.

\subsection{O MEC APLICADO A PROBLEMAS ANISOTRÓPICOS}

O MEC é uma técnica numérica alternativa para a resolução de problemas dentro dos diversos campos da Engenharia. Seu princípio básico é a transformação das equações diferenciais, que regem determinado problema físico, em equações integrais de contorno com variáveis de contorno nos integrandos. De posse da equação integral de contorno, o MEC consiste em se adotar uma aproximação numérica para ela, obtendo-se um conjunto de equações algébricas lineares que permitem a resolução de diversos problemas cujas soluções não são conhecidas explicitamente.

$\mathrm{Na}$ literatura especializada, existem diversos trabalhos escritos sobre a aplicação de equações integrais em problemas das teorias de potencial e de Elasticidade. Um dos primeiros registros sobre a utilização das equações integrais remontam a $\mathrm{Abel}^{1}$ que usou uma equação integral para resolver o problema chamado "pêndulo isócrono”. A representação integral para problemas da elastostática foi estabelecida por Somigliana², ficando-se conhecida como Identidade Somigliana.

Os métodos numéricos modernos de equações integrais de contorno estão diretamente relacionados ao trabalho de FREDHOLM (1903), que utilizou equações integrais discretizadas para tratar problemas de potencial, formando assim, a base para a abordagem indireta do MEC. Essa abordagem é conhecida como indireta porque faz uso

\footnotetext{
${ }^{1}$ N. H. Abel, Ouevres completes. Norvegien Christiania, 1881, vol.1, 621p.

${ }^{2}$ C. Somigliana . Sopra l'equilibrio di um corpo elástico isótropo. Il nuovo Cimento(Ser. 3),1886,p.17-20.
} 
de fontes fictícias aplicadas ao contorno, mas que podem ser utilizadas no cálculo de grandezas físicas como deslocamentos e tensões. Nesse contexto, citam-se, por exemplo, os trabalhos de MUSKHELISHVILI (1953), MIKHLIN (1957) e KUPRADZE (1965).

A abordagem direta do MEC, assim chamada pelo fato das variáveis físicas do problema (deslocamentos e forças de superfície) serem usadas diretamente na equação integral, foi apresentada nos trabalhos de RIZZO (1967) e de CRUSE \& RIZZO (1968). O trabalho de RIZZO (1967) foi o primeiro a explorar a analogia entre as teorias de potencial e da Elasticidade clássica, procurando-se uma forma numérica de se resolver o problema. Ele usou elementos retilíneos para discretizar o contorno onde as variáveis assumiam valores constantes em cada elemento.

A partir desses trabalhos pioneiros o MEC se desenvolveu de forma bastante rápida, sendo atualmente um método bem estabelecido com aplicações em diversas áreas da Engenharia, como pode ser constatado nos trabalhos de CRUSE (1987) e de BREBBIA \& DOMINGUEZ (1989).

Com relação ao estudo da plasticidade isotrópica com o MEC, os primeiros trabalhos foram o de RICARDELLA (1973) e MENDELSON (1973). As formulações inicialmente propostas apresentavam algumas incorreções que foram contornadas mais tarde por BUI (1978) por meio de conceitos sobre derivada de uma integral singular. Outros trabalhos que abordaram plasticidade com o MEC podem ainda ser citados, como por exemplo, VENTURINI (1982), TELLES \& CARRER (1994), CHUEIRI (1994) e FUDOLI (1999).

No entanto, apesar do avanço alcançado, uma das grandes dificuldades inerente ao MEC consiste na determinação da solução fundamental do problema. A solução fundamental, muitas vezes chamada de Função de Green, vem a ser a resposta da variável, ou das variáveis, da equação diferencial do problema físico em um ponto do domínio infinito devido à aplicação de uma força unitária em um outro ponto do mesmo domínio. Muitos dos problemas clássicos da Engenharia tem soluções fundamentais conhecidas, outros não e alguns apresentam soluções muito complexas que inviabilizam a aplicação do método. Para a análise de corpos elásticos isotrópicos, a primeira solução fundamental foi desenvolvida por Lord Kelvin ${ }^{3}$.

\footnotetext{
${ }^{3}$ W. Thompson (Lord Kelvin): Note on the integration of the equations of equilibrium of an elastic solid. Cambridge and Dublin Mathematical Journal, 3, 87- 89,1848.
} 
Abordando-se particularmente problemas anisotrópicos com o MEC, pode-se dizer que não é um tema muito explorado. O progresso alcançado na análise desses problemas no decorrer dos anos tem sido relativamente menor do que o alcançado na Mecânica isotrópica e ainda, na maioria das vezes, esse progresso originou somente aplicações destinadas à análise linear de meios ortotrópicos ou transversalmente isotrópicos.

A primeira formulação do MEC para problemas elásticos lineares planos, não isotrópicos, foi desenvolvida por RIZZO \& SHIPPY (1970). Utilizando-se da solução fundamental para a análise de problemas ortotrópicos proposta por GREEN (1943), baseada somente em variáveis reais, aplicaram a abordagem direta de solução para o estudo de tensões em alguns exemplos de sólidos ortotrópicos. Foram utilizados elementos constantes para aproximar as variáveis e a geometria do problema.

A partir desse trabalho, surgiram outros que aplicaram também a mesma solução fundamental de RIZZO \& SHIPPY (1970) com a finalidade de se estudar sólidos ortotrópicos planos, podendo ser citados BENJUMEA \& SIKARSKIE (1972), MAHAJERIN \& SIKARSKIE (1986), VABLE \& SIKARSKIE (1988) e PADHI et al. (2000).

A análise de sólidos anisotrópicos planos, possuindo anisotropia geral, através do MEC teve início com o trabalho de CRUSE \& SWEDLOW (1971) que, utilizando funções de variáveis complexas e o formalismo elástico anisotrópico de LEKHNITSKII (1963), propuseram uma solução fundamental bi-dimensional que tem sido bastante utilizada nas mais diferentes aplicações do MEC em anisotropia, podendo ser citados, de forma mais detalhada, alguns trabalhos a seguir.

SNYDER \& CRUSE (1975) determinaram fatores de intensidade de tensão em chapas elásticas anisotrópicas, contendo uma trinca, utilizando uma solução fundamental anisotrópica própria para trincas, mas, desenvolvida a partir da solução fundamental com variáveis complexas de CRUSE \& SWEDLOW (1971). Em seu estudo, adotando aproximação constante para as variáveis, constataram uma diferença próxima de $10 \%$ entre os fatores de intensidade de tensão das chapas analisadas (ortotrópicas) com os de chapas isotrópicas.

No campo da Mecânica da Fratura, podem ainda ser citados outros trabalhos. TAN \& GAO (1992) analisaram sólidos anisotrópicos planos com concentrações de tensões e trincas. SOLLERO \& ALIABADI (1993) determinaram fatores de intensidade de tensão em chapas anisotrópicas bidimensionais. Algumas aplicações são 
apresentadas para laminados simétricos unidirecionais constituídos de materiais poliméricos reforçados por fibras. ALBUQUERQUE (2001) e ALBUQUERQUE et al. (2002) aplicaram a formulação do MEC para análise de problemas dinâmicos lineares envolvendo estruturas constituídas de materiais anisotrópicos.

Analisando alguns exemplos de sólidos planos com anisotropia geral, DEB \& BANERJEE (1990) utilizaram a solução fundamental com variáveis complexas para estudar as tensões e as deformações em corpos considerando a presença de forças de volume (força da gravidade e força centrífuga). Os mesmos procedimentos de solução foram utilizados por DEB (1996) para a análise de corpos anisotrópicos bidimensionais sujeitos a efeitos de temperatura.

LEE \& MAL (1990) apresentaram uma formulação para o MEC, para a análise de meios anisotrópicos planos, onde as equações integrais são discretizadas num plano complexo, diferenciando-se assim das formulações usuais. As incógnitas do problema são assumidas como funções lineares de uma variável complexa, em cada elemento de contorno, e as integrações são realizadas de forma exata para contornos arbitrários sem a necessidade de integrações numéricas, constituindo assim, a vantagem do método proposto.

Procurando melhorar as respostas obtidas com o MEC no cálculo de tensões no contorno, e próximas a ele, de sólidos anisotrópicos bidimensionais, RAJU et al. (1996) apresentaram uma formulação baseada nos gradientes dos deslocamentos (deformações) e nas forças de superfície.

Nesse método, chamado de gradiente do deslocamento modificado (Okada et al. (1988) apud RAJU et al. (1996)), a formulação tradicional do MEC é utilizada para se determinar os deslocamentos e forças de superfícies em todos os nós do contorno. Depois, todos os dados do contorno são então usados para se determinar os gradientes dos deslocamentos em cada nó do contorno. Esses gradientes e as forças são então usados no método do gradiente para se determinar as deformações em pontos internos do corpo, e as tensões são então calculadas utilizando as relações constitutivas.

A solução fundamental de CRUSE \& SWEDLOW (1971) é também utilizada na análise transiente de sólidos anisotrópicos piezoelétricos, citando-se, por exemplo, o trabalho de KÖGL \& GAUL (2000). AZIS \& CLEMENTS (2001), estudando deformações de sólidos anisotrópicos não homogêneos, utilizaram uma solução desenvolvida a partir da solução de CRUSE \& SWEDLOW (1971). 
Assim, em vista de todos esses trabalhos comentados, é possível constatar a grande aplicabilidade da solução fundamental anisotrópica com variáveis complexas para a análise de problemas planos com anisotropia geral. Devido a isso, no presente trabalho, essa solução fundamental será utilizada de maneira original em problemas não lineares e mais detalhes sobre ela são apresentados no decorrer do trabalho.

Antes, porém, é de interesse ainda neste item, apresentar algumas referências cujos autores tiveram o propósito de analisar problemas anisotrópicos planos e também, tridimensionais utilizando-se de outras soluções fundamentais. Dessa forma, a seguir são feitos alguns comentários sobre outros trabalhos que versaram o assunto anisotropia com aplicações do MEC.

Para a análise elastostática tridimensional, a primeira aplicação do MEC foi feita por VOGEL \& RIZZO (1973) utilizando uma solução fundamental proposta por SYNGE (1957) escrita somente em função de variáveis reais:

$$
G_{i j}(X, \xi)=\frac{1}{8 \pi^{2} \cdot|X-\xi|} \cdot \oint_{|\gamma|=1} K_{i j}^{-1}(\gamma) d s \quad(i, j=1,2,3)
$$

onde a integral de linha é tomada sobre um círculo unitário no plano normal ao vetor $(X$ - $\xi$ ) e passando através de $X$, e o integrando $\left(K_{i j}\right)$ é definido como segue (DEB, 1996):

$$
K_{i j}^{-1}(\gamma)=\left[C_{i j k l} \cdot \gamma_{k} \cdot \gamma_{l}\right]^{-1}
$$

onde o tensor $C_{i j k l}$ representa o tensor dos coeficientes de rigidez.

No entanto, para o caso geral de anisotropia, a integração numérica da Equação (2.1) demandava muito esforço computacional. Assim, WILSON \& CRUSE (1978) formularam um esquema de interpolação que evitava a utilização da Equação (2.1) em todos os pontos de Gauss. Outros autores, como DEB et al. (1991) e SALES \& GRAY (1998), procuraram adotar aproximações mais eficientes, baseadas em técnicas matemáticas como transformação em séries finitas, para a integração numérica da Equação (2.1).

BREBBIA \& DOMINGUEZ (1989) propuseram uma solução fundamental deduzida a partir da solução fundamental isotrópica de Kelvin, evitando-se, dessa maneira, para problemas tridimensionais, a integração numérica para se determinar a função de Green. 
A técnica consiste em expressar as constantes anisotrópicas como uma média dos valores das constantes isotrópicas mais um resíduo, que por sua vez, é transformado num termo de domínio da equação integral e pode ser tratado, por exemplo, pelo método da reciprocidade dual.

Utilizando essa solução fundamental, seguiram-se os trabalhos de SCHCLAR \& PARTRIDGE (1993) e SCHCLAR (1994), na análise de diversos problemas anisotrópicos tridimensionais, e PEREZ \& WROBEL (1996) no estudo de problemas planos. Esta técnica subtrai do MEC sua principal vantagem para problemas lineares, isto é, necessita da introdução de aproximações de domínio.

Para meios infinitos transversalmente isotrópicos, PAN \& CHOU (1976) obtiveram uma solução fundamental utilizando-se de três funções potenciais de deslocamentos. Essa solução foi empregada por PAN \& AMADEI (1996), no estudo de sólidos sujeitos a ação da gravidade e também, por DING \& LIANG (1999) na análise de sólidos piezoelétricos transversalmente isotrópicos.

E por fim, concluindo este item sobre soluções fundamentais anisotrópicas, é de interesse ainda apresentar alguns trabalhos baseados em um outro ramo da elasticidade anisotrópica conhecido como formalismo de Stroh (ESHELBY et al., 1952; STROH, 1958; e STROH, 1962).

Segundo TING (1996), o formalismo anisotrópico de LEKHNITSKII (1963) tem sido ao longo dos anos muito explorado entre os pesquisadores da área de Engenharia enquanto que, o formalismo de Stroh, é bem conhecido entre aqueles que fazem pesquisa nas áreas de Matemática aplicada, Física e Ciência dos Materiais.

Porém, na última década, esse formalismo anisotrópico tem despertado interesse de pesquisadores engenheiros devido sua elegância matemática e potencialidade de resolução de diversos problemas, como por exemplo, piezoeletricidade anisotrópica, eletromagnetismo, trincas e outros (TING, 2000).

De uma forma geral, pode-se dizer que o formalismo de Stroh transforma um dado problema anisotrópico em um problema de autovalores e autovetores (BARNETT \& LOTHE, 1973):

$$
N \cdot \xi=p \cdot \xi
$$

onde $N$ é uma matriz real 6 x 6 dependente das constantes elásticas de rigidez do material, diferente do formalismo de LEKHNITSKII (1963) que utiliza as constantes de 
compliância; $p$ são 6 autovalores consistindo de 3 pares complexos conjugados e $\xi$ são os autovetores associados a $p$.

Com esses resultados e seguindo o equacionamento matemático do formalismo (ESHELBY et al. (1952), STROH (1958), STROH (1962) e TING (1996)), obtém-se as soluções para os problemas da elasticidade anisotrópica, estando entre eles, a determinação de soluções fundamentais.

Com a difusão do formalismo de Stroh, alguns trabalhos têm sido desenvolvidos com o propósito de se obter soluções fundamentais anisotrópicas. WANG (1994) obteve várias soluções fundamentais considerando um formalismo de Stroh generalizado. Essas soluções foram implementadas com o MEC para problemas tridimensionais por TONON et al. (2001). Uma solução desenvolvida por Yuan \& Yang apud PAN et al. (2001), foi implementada por PAN et al. (2001) para a análise de tensões em torno de furos em laminados compósitos.

Em TING (1996) é possível encontrar soluções fundamentais para diversos problemas anisotrópicos como, por exemplo, trincas em sólidos compósitos, inclusões elípticas e problemas elásticos em semi-espaços infinitos. Outras soluções ainda podem ser encontradas em CHIU \& WU (1998), WU (1998) e PAN \& YUAN (2000).

Com isso, tendo-se apresentado diversos trabalhos sobre soluções fundamentais anisotrópicas, é importante salientar novamente, que na presente pesquisa, a solução fundamental a ser utilizada é a solução de variáveis complexas de CRUSE \& SWEDLOW (1971) deduzida a partir do formalismo de LEKHNITSKII (1963), pois, como já comentado, ela é potencialmente eficiente para os propósitos deste trabalho.

E por fim, deve-se também comentar que entre os diversos trabalhos consultados não se encontrou nenhum que se assemelha, seja no assunto enfocado ou na metodologia proposta, ao presente trabalho, garantindo assim, seu caráter original. Salienta-se mais uma vez, no sentido de se esclarecer os pontos inovadores do trabalho, que as análises plásticas e viscoplásticas com anisotropia geral pelo MEC são assuntos inexplorados (SUN et al., 2002) e serão originalmente aqui enfocados. 


\section{MEIOS ANISOTRÓPICOS - CONSIDERAÇÕES GERAIS E CRITÉRIOS DE RESISTÊNCIA}

Neste capítulo são apresentadas considerações gerais sobre meios anisotrópicos, suas relações constitutivas, destacando-se aspectos básicos que as diferenciam das relações dos meios isotrópicos, e também, a solução fundamental empregada é brevemente descrita. É feita ainda uma revisão da literatura sobre critérios de resistência específicos para materiais não-isotrópicos no intuito de se definir qual critério é o mais apropriado para as análises, em meios com anisotropia geral, propostas nesta pesquisa.

\subsection{GENERALIDADES}

A maioria dos materiais estruturais exibe algum grau de anisotropia. Um material como a madeira é naturalmente anisotrópico, outros, como os compósitos, são anisotrópicos devido ao processo de fabricação.

De um modo geral, segundo LEKHNITSKII (1963), todos os materiais podem ser classificados, por um lado, em homogêneos e não homogêneos, e por outro lado, em isotrópicos e anisotrópicos. Em meios homogêneos as propriedades de elasticidade são invariantes para todos os pontos e, para meios não homogêneos, essas propriedades não se apresentam as mesmas.

LEKHNITSKII (1963) classifica como isotrópico o sólido cujas propriedades de elasticidade são constantes para quaisquer direções estabelecidas a partir de um 
determinado ponto, ou seja, são invariantes para todas as transformações de coordenadas. Um sólido anisotrópico, como um material compósito reforçado por fibras, exibe diferentes propriedades de elasticidade para direções diferentes associadas a um dado ponto. As direções nas quais as propriedades de elasticidade se mantém são denominadas direções elasticamente equivalentes ou direções principais de elasticidade.

Materiais anisotrópicos artificiais, como os compósitos estruturais, foram originalmente desenvolvidos para a indústria aeroespacial com a vantagem de oferecerem elevadas propriedades de rigidez e resistência quando comparadas ao seu peso próprio (SCHCLAR, 1994). Atualmente os compósitos têm encontrado um campo de aplicações maior do que a indústria aeroespacial, podendo-se citar, por exemplo, aplicações nas indústrias navais, automotivas, eletroeletrônicas e na construção civil (não esquecendo que o concreto armado é um dos mais antigos materiais compósitos artificiais).

Tratando-se especificamente sobre a análise de estruturas constituídas de materiais anisotrópicos, pode-se dizer que surgem complicações adicionais quando comparadas com as estruturas isotrópicas. Isso se deve ao fato de que as relações constitutivas, ou seja, as relações entre tensões e deformações, em sólidos anisotrópicos são mais complexas sendo representadas por um tensor constitutivo mais completo, aumentandose assim, o número de variáveis do problema.

\subsection{LEIS CONSTITUTIVAS PARA MATERIAIS ELÁSTICOS ANISOTRÓPICOS}

Utilizando-se das propriedades de energia de deformação, pode-se estabelecer formulações das leis constitutivas para diversas classes de materiais elásticos. Seja, então, a função energia de deformação, caracterizada pela seguinte série polinomial (CHEN \& SALEEB, 1982):

$$
U_{0}=C_{0} \delta_{i j}+\kappa_{i j} \varepsilon_{i j}+\varpi_{i j k l} \varepsilon_{i j} \varepsilon_{k l}
$$

onde: $C_{0}, \kappa_{i j}$, $\varpi_{i j k l}$ são funções constantes. 
Baseando-se nos conceitos de energia de deformação, onde a energia de deformação tem um valor estacionário em relação ao tensor das deformações, é possível arbitrar para essa constante $\mathrm{C}_{0}$ um valor igual a zero. Aplicando-se a expressão $\sigma_{i j}=\frac{\partial U_{0}}{\partial \varepsilon_{i j}}$, com $U_{0}$ de acordo com a equação (3.1) obtém-se:

$$
\sigma_{i j}=\kappa_{i j}+\left(\varpi_{i j k l}+\varpi_{k l i j}\right) \varepsilon_{k l}
$$

Para o caso relativo ao "estado natural” no qual as tensões e as deformações estão vinculadas a todo o sólido e, também, estão atuando simultaneamente no sólido, consegue-se escrever que:

$$
\left(\sigma_{i j}\right)_{0}=\left(\frac{\partial U_{0}}{\partial \varepsilon_{i j}}\right)_{0}=0
$$

onde a notação entre parênteses com índice 0 , aponta para um estado natural com tensões e deformações nulas. Assim, $\kappa_{i j}$ vale zero. Denominando-se $\left(\varpi_{i j k l}+\varpi_{k l i j}\right)$ de $C_{i j k l}$, pode-se escrever que:

$$
\sigma_{i j}=C_{i j k l} \varepsilon_{k l}
$$

O tensor $C_{i j k l}$ é chamado de tensor de constantes de elasticidade. Pode-se ainda exprimir a lei constitutiva (3.4) numa forma tensorial alternativa, admitindo-se naturalmente que $\left|C_{i j k l}\right| \neq 0$, por intermédio de:

$$
\varepsilon_{i j}=S_{i j k l} \sigma_{k l}
$$

onde: $S_{i j k l}$ é denominado tensor de compliância ou matriz de flexibilidade

Do ponto de vista formal, os tensores $C_{i j k l}$ e $S_{i j k l}$ são constituídos de 81 (oitenta e um) elementos, já que os índices i, j, k, $l$ variam de 1 a 3. O tensor $C_{i j k l}$ relaciona cada um dos nove componentes do tensor de deformação a cada uma das nove componentes do tensor de tensão. O tensor $S_{i j k l}$, por outro lado, faz esta inter-relação de maneira inversa.

A partir do fato associado às seguintes derivações:

$$
\frac{\partial \sigma_{i j}}{\partial \varepsilon_{k l}}=\frac{\partial^{2} U_{o}}{\partial \varepsilon_{k l} \partial \varepsilon_{i j}}=C_{i j k l}
$$


e

$$
\frac{\partial^{2} U_{0}}{\partial \varepsilon_{k l} \partial \varepsilon_{i j}}=\frac{\partial^{2} U_{0}}{\partial \varepsilon_{i j} \partial \varepsilon_{k l}}
$$

pode-se concluir que $C_{i j k l}=C_{k l i j}$.

Retomando-se a expressão (3.4), o tensor $C_{\mathrm{ijk} l}$ acena para a existência de 81 elementos. Não obstante, deve-se lembrar que tanto o tensor das tensões como o tensor das deformações são simétricos. Direcionando-se estes conceitos à Equação (3.4), temse:

Simetria do tensor de tensões $\left(\sigma_{i j}\right)$ :

$$
\sigma_{i j}=C_{i j k l} \varepsilon_{k l}=\sigma_{j i}=C_{j i k l} \varepsilon_{k l}
$$

e daí:

$$
C_{i j k l}=C_{j i k l}
$$

Por outro lado, pela simetria do tensor das deformações $\left(\varepsilon_{i j}\right)$ :

$$
\sigma_{i j}=C_{i j k l} \varepsilon_{k l}=C_{i j l k} \varepsilon_{l k}
$$

e portanto:

$$
C_{i j k l}=C_{i j l k}
$$

Nesse sentido, dos 81 elementos, ao se aplicar (3.9), sobram 54 elementos e quando se vincula (3.11), sobram 36 elementos. Entretanto, o tensor $C_{i j k l}$ é simétrico em relação aos pares $(i, j)$ e $(k, l)$, Equação (3.7). Com efeito, dos 36 elementos, tem-se, assim, somente 21 elementos do tensor constitutivo $C_{i j k l}$. As mesmas considerações se aplicam ao tensor de flexibilidade $S_{i j k l}$.

\subsubsection{Representação das Leis Constitutivas}

Tendo-se em vista a simetria dos tensores de tensão e de deformação, é possível constatar as identidades: 


$$
\begin{array}{ll}
\sigma_{12}=\sigma_{21} & \varepsilon_{12}=\varepsilon_{21} \\
\sigma_{13}=\sigma_{31} & \varepsilon_{13}=\varepsilon_{31} \\
\sigma_{23}=\sigma_{32} & \varepsilon_{23}=\varepsilon_{32}
\end{array}
$$

Dessa forma, as nove equações que caracterizam as expressões tensoriais das leis constitutivas podem ser condensadas em seis equações com seis termos cada uma.

Neste sentido, a lei constitutiva (3.4) pode também ser escrita da seguinte maneira:

$$
\left\{\begin{array}{l}
\sigma_{11} \\
\sigma_{22} \\
\sigma_{33} \\
\sigma_{12} \\
\sigma_{23} \\
\sigma_{31}
\end{array}\right\}=\left[\begin{array}{llllll}
C_{1111} & C_{1122} & C_{1133} & C_{1112} & C_{1123} & C_{1131} \\
& C_{2222} & C_{2233} & C_{2212} & C_{2223} & C_{2231} \\
& & C_{3333} & C_{3312} & C_{3323} & C_{3331} \\
& & & C_{1212} & C_{1223} & C_{1231} \\
& & & & C_{2323} & C_{2331} \\
\operatorname{Sim} . & & & & & C_{3131}
\end{array}\right] \cdot\left\{\begin{array}{c}
\varepsilon_{11} \\
\varepsilon_{22} \\
\varepsilon_{33} \\
2 \varepsilon_{12} \\
2 \varepsilon_{23} \\
2 \varepsilon_{31}
\end{array}\right\}
$$

ou simplesmente, em forma matricial:

$$
\sigma=C \varepsilon
$$

De modo análogo, $\varepsilon_{i j}=S_{i j k l} \sigma_{k l}$ fica sendo:

$$
\varepsilon=S \sigma
$$

ou:

$$
\left\{\begin{array}{l}
\varepsilon_{11} \\
\varepsilon_{22} \\
\varepsilon_{33} \\
2 \varepsilon_{12} \\
2 \varepsilon_{23} \\
2 \varepsilon_{31}
\end{array}\right\}=\left[\begin{array}{llllll}
S_{1111} & S_{1122} & S_{1133} & 2 S_{1112} & 2 S_{1123} & 2 S_{1131} \\
& S_{2222} & S_{2233} & 2 S_{2212} & 2 S_{2223} & 2 S_{2231} \\
& & S_{3333} & 2 S_{3312} & 2 S_{3323} & 2 S_{3331} \\
& & & 4 S_{1212} & 4 S_{1223} & 4 S_{1231} \\
& & & & 4 S_{2323} & 4 S_{2331} \\
\text { Sim. } & & & & & 4 S_{3131}
\end{array}\right] \cdot\left\{\begin{array}{l}
\sigma_{11} \\
\sigma_{22} \\
\sigma_{33} \\
\sigma_{12} \\
\sigma_{23} \\
\sigma_{31}
\end{array}\right\}
$$

Observa-se que os valores $2 \varepsilon_{12}, 2 \varepsilon_{23}$ e $2 \varepsilon_{31}$ podem ser substituídos por $\gamma_{12}$, $\gamma_{23}, \gamma_{31}$ e as tensões $\sigma_{12}, \sigma_{23}, \sigma_{31}$ por $\tau_{12}, \tau_{23}, \tau_{31}$. 
Introduzindo-se uma notação reduzida, apresentada por TING (1996):

$$
\begin{array}{ll}
\sigma_{11}=\sigma_{1}, & \sigma_{23}=\sigma_{4} \\
\sigma_{22}=\sigma_{2}, & \sigma_{31}=\sigma_{5} \\
\sigma_{33}=\sigma_{3}, & \sigma_{12}=\sigma_{6} \\
\varepsilon_{11}=\varepsilon_{1}, & 2 \varepsilon_{23}=\varepsilon_{4} \\
\varepsilon_{22}=\varepsilon_{2}, & 2 \varepsilon_{31}=\varepsilon_{5} \\
\varepsilon_{33}=\varepsilon_{3}, & 2 \varepsilon_{12}=\varepsilon_{6}
\end{array}
$$

Assim, a lei constitutiva (3.13) pode ser escrita como:

$$
\sigma_{\alpha}=C_{\alpha \beta} \varepsilon_{\beta}
$$

e a Equação (3.16):

$$
\varepsilon_{\alpha}=S_{\alpha \beta} \sigma_{\beta}
$$

A transformação entre $C_{i j k l}$ e $C_{\alpha \beta}$ é efetuada substituindo-se os subscritos ij (ou $\mathrm{kl}$ ) por $\alpha$ (ou $\beta$ ) usando-se as seguintes regras:

TABELA 3.1: Regras de transformação entre ij (ou kl) e $\alpha$ (ou $\beta$ ) (TING, 1996).

\begin{tabular}{ccc}
\hline ij (ou kl) & & $\alpha($ ou $\beta)$ \\
\hline 11 & $\Leftrightarrow$ & 1 \\
\hline 22 & $\Leftrightarrow$ & 2 \\
\hline 33 & $\Leftrightarrow$ & 3 \\
\hline 23 ou 32 & $\Leftrightarrow$ & 4 \\
\hline 31 ou 13 & $\Leftrightarrow$ & 5 \\
\hline 12 ou 21 & $\Leftrightarrow$ & 6 \\
\hline
\end{tabular}

Para a transformação entre $S_{i j k l}$ e $S_{\alpha \beta}$ utiliza-se as mesmas regras, porém considerando-se:

$$
\begin{array}{ll}
S_{i j k l}=S_{\alpha \beta} & \text { se } \alpha \text { e } \beta \leq 3 ; \\
2 S_{i j k l}=S_{\alpha \beta} & \text { se } \alpha \text { ou } \beta \leq 3 ;
\end{array}
$$




$$
4 S_{i j k l}=S_{\alpha \beta} \quad \text { se } \alpha \text { e } \beta>3 .
$$

Assim, é possível escrever:

$$
\left\{\begin{array}{l}
\sigma_{1} \\
\sigma_{2} \\
\sigma_{3} \\
\sigma_{4} \\
\sigma_{5} \\
\sigma_{6}
\end{array}\right\}=\left[\begin{array}{cccccc}
C_{11} & C_{12} & C_{13} & C_{14} & C_{15} & C_{16} \\
& C_{22} & C_{23} & C_{24} & C_{25} & C_{26} \\
& & C_{33} & C_{34} & C_{35} & C_{36} \\
& & & C_{44} & C_{45} & C_{46} \\
& & & & C_{55} & C_{56} \\
\text { Sim. } & & & & & C_{66}
\end{array}\right] \cdot\left\{\begin{array}{l}
\varepsilon_{1} \\
\varepsilon_{2} \\
\varepsilon_{3} \\
\varepsilon_{4} \\
\varepsilon_{5} \\
\varepsilon_{6}
\end{array}\right\}
$$

e também,

$$
\left\{\begin{array}{l}
\varepsilon_{1} \\
\varepsilon_{2} \\
\varepsilon_{3} \\
\varepsilon_{4} \\
\varepsilon_{5} \\
\varepsilon_{6}
\end{array}\right\}=\left[\begin{array}{llllll}
S_{11} & S_{12} & S_{13} & S_{14} & S_{15} & S_{16} \\
& S_{22} & S_{23} & S_{24} & S_{25} & S_{26} \\
& & S_{33} & S_{34} & S_{35} & S_{36} \\
& & & S_{44} & S_{45} & S_{46} \\
& & & & S_{55} & S_{56} \\
\operatorname{Sim} . & & & & & S_{66}
\end{array}\right] \cdot\left\{\begin{array}{l}
\sigma_{1} \\
\sigma_{2} \\
\sigma_{3} \\
\sigma_{4} \\
\sigma_{5} \\
\sigma_{6}
\end{array}\right\}
$$

Considerando-se agora a relação constitutiva inversa (Equação 3.22), expressa pelo tensor de flexibilidade $S_{i j}$, pode-se expandi-la escrevendo-se os coeficientes elásticos em termos das constantes de engenharia e assim, tem-se:

$$
\begin{aligned}
& \varepsilon_{x}=\frac{1}{E_{x x}}\left(\sigma_{x}-v_{y x} \sigma_{y}-v_{z x} \sigma_{z}+\eta_{y z, x} \tau_{y z}+\eta_{z x, x} \tau_{x z}+\eta_{x y, x} \tau_{x y}\right) \\
& \varepsilon_{y}=\frac{1}{E_{y y}}\left(-v_{x y} \sigma_{x}+\sigma_{y}-v_{z y} \sigma_{z}+\eta_{y z, y} \tau_{y z}+\eta_{z x, y} \tau_{x z}+\eta_{x y, y} \tau_{x y}\right) \\
& \varepsilon_{z}=\frac{1}{E_{z z}}\left(-v_{x z} \sigma_{x}-v_{y x} \sigma_{y}+\sigma_{z}+\eta_{y z, z} \tau_{y z}+\eta_{z x, z} \tau_{x z}+\eta_{x y, z} \tau_{x y}\right) \\
& \gamma_{y z}=\frac{1}{G_{y z}}\left(\eta_{x, y z} \sigma_{x}+\eta_{y, y z} \sigma_{y}+\eta_{z, y z} \sigma_{z}+\tau_{y z}+\mu_{z x, y z} \tau_{x z}+\mu_{x y, y z} \tau_{x y}\right) \\
& \gamma_{x z}=\frac{1}{G_{x z}}\left(\eta_{x, z x} \sigma_{x}+\eta_{y, z x} \sigma_{y}+\eta_{z, z x} \sigma_{z}+\mu_{y z, z x} \tau_{y z}+\tau_{x z}+\mu_{x y, z x} \tau_{x y}\right) \\
& \gamma_{x y}=\frac{1}{G_{x y}}\left(\eta_{x, x y} \sigma_{x}+\eta_{y, x y} \sigma_{y}+\eta_{z, x y} \sigma_{z}+\mu_{y z, x y} \tau_{y z}+\mu_{z x, x y} \tau_{x z}+\tau_{x y}\right)
\end{aligned}
$$

Nas equações anteriores, $E_{i j}$ são os módulos de Elasticidade longitudinais nas direções dos eixos $x, y, z ; G_{i j}$, são os módulos de Elasticidade transversais para os planos 
paralelos aos planos coordenados; $v_{i j}$, são os coeficientes de Poisson. As outras constantes que aparecem nas equações são nulas para um corpo isotrópico (ou ortotrópico), mas, importantes para um corpo anisotrópico, pois quantificam as influências de tensões normais em deformações tangenciais e de tensões tangenciais em deformações normais (MASCIA, 1991; VANALLI , 2001).

As constantes $\mu_{i j, j k}$ são chamadas de coeficientes de Chentsov. Eles caracterizam distorções em planos paralelos aos planos coordenados produzidas por tensões tangenciais agindo em planos paralelos aos outros planos coordenados. As constantes $\eta_{i j, k}$ são chamadas, de acordo com LEKHNITSKII (1963), de coeficientes de influência mútua de $1^{\text {a }}$ espécie. Eles caracterizam extensões nas direções dos eixos coordenados “ $k$ ” produzidas por tensões tangenciais agindo nos planos coordenados “ij”. E por fim, tem-se os coeficientes de influência mútua de $2^{\text {a }}$ espécie, $\eta_{i, j k}$ que expressam deformações tangenciais nos planos coordenados “ $j k$ ”, causadas pelas tensões normais que atuam nos eixos coordenados “ $i$ ”.

\subsubsection{Classificação dos Materiais segundo o Número de Planos de Simetria Elástica}

Se um meio anisotrópico apresenta algum tipo de simetria, as suas propriedades de elasticidade também a exibem. A simetria elástica expressa o fato de que em cada ponto do sólido existem direções simétricas equivalentes com respeito às propriedades elásticas. Se há simetria das propriedades elásticas de um meio anisotrópico, então as leis constitutivas para ele podem ser simplificadas, ou seja, podem ocorrer redução de coeficientes nos tensores $S_{i j}$ e $C_{i j}$. LEKHNITSKII (1963), procurando realizar tais simplificações, desenvolve as leis constitutivas em relação a dois sistemas simétricos de coordenadas e compara as expressões obtidas, identificando assim, as simetrias existentes.

Dessa maneira, ao se fazer considerações da presença de planos de simetria em sólidos anisotrópicos, surgem diversas classes de materiais diferenciadas pela quantidade de elementos distintos e independentes nos tensores constitutivos. Com isso, apresenta-se a seguir, alguns casos básicos de simetria elástica. 


\subsubsection{Material com Simetria Elástica em um Plano}

Considerando-se um plano $\pi$, referido a um sistema de coordenadas $x_{i}$, conforme se mostra na Figura 3.1:

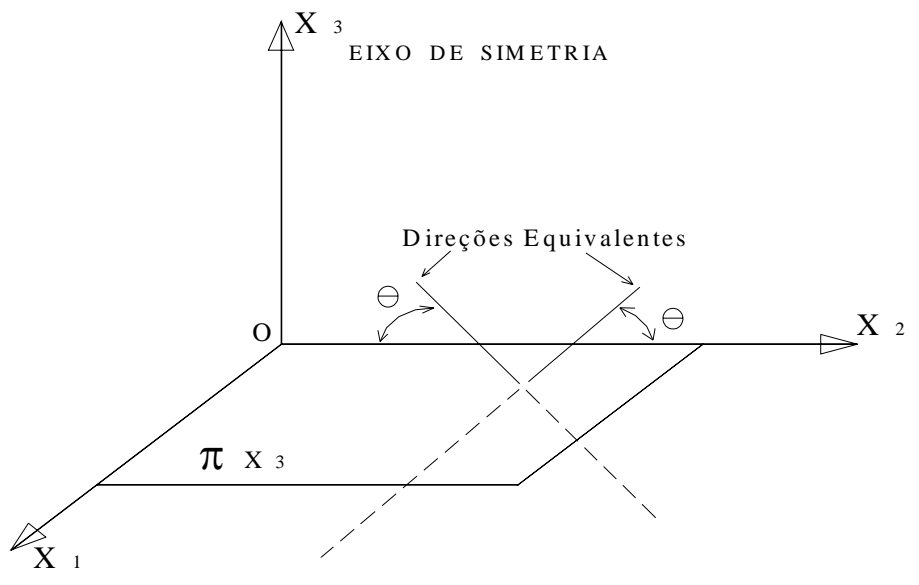

FIGURA 3.1 - Simetria elástica em 1 (um) plano.

o plano $x_{1}-x_{2}$ é de simetria elástica, ou seja, duas direções quaisquer passando por um ponto nesse plano são equivalentes no que concerne às propriedades de elasticidade. A direção normal a este plano é chamada de direção principal de elasticidade.

Promovendo-se rotações de $180^{\circ}$ em torno do eixo $x_{3}$ (Figura 3.2), no intuito de se chegar às simplificações:

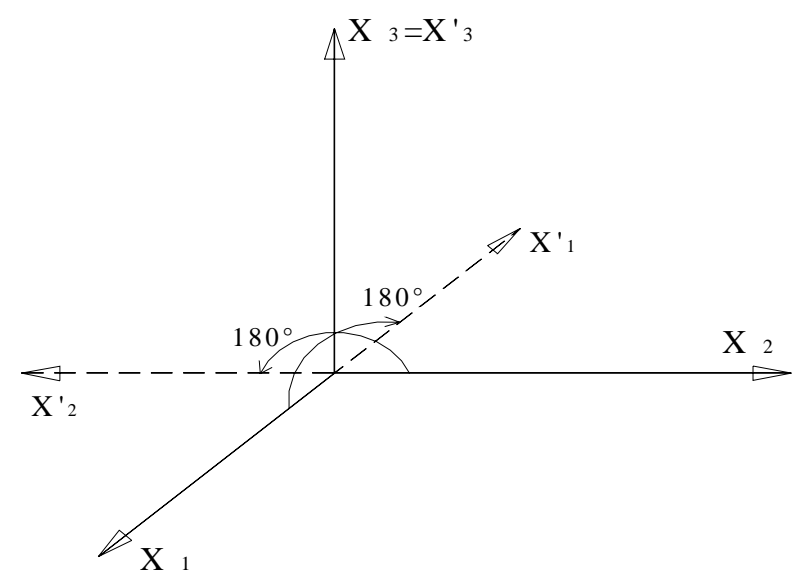

FIGURA 3.2 - Rotação de $180^{\circ}$ em torno do eixo $\mathrm{x}_{3}$.

têm-se os seguintes cossenos diretores:

$$
l_{i j}=\left[\begin{array}{ccc}
-1 & 0 & 0 \\
0 & -1 & 0 \\
0 & 0 & -1
\end{array}\right]
$$


Com o uso de transformação tensorial, para transformação de propriedades de elasticidade, apresentada por LEKHNITSKII (1963) obtém-se para o coeficiente $S_{11}$ :

$$
S_{11}^{\prime}=q_{1 m} q_{1 n} S_{m n}
$$

onde os coeficientes $q_{i j}$ são dependentes dos cossenos diretores das transformações, sendo apresentados na Tabela 3.2:

TABELA 3.2 - Coeficientes $q_{i j}$

\begin{tabular}{c|c|c|c|c|c|c}
\hline & 1 & 2 & 3 & 4 & 5 & 6 \\
\hline 1 & $l_{11}^{2}$ & $l_{12}^{2}$ & $l_{13}^{2}$ & $l_{11} l_{12}$ & $l_{12} l_{13}$ & $l_{31} l_{11}$ \\
\hline 2 & $l_{21}^{2}$ & $l_{22}^{2}$ & $l_{23}^{2}$ & $l_{22} l_{21}$ & $l_{23} l_{22}$ & $l_{23} l_{21}$ \\
\hline 3 & $l_{31}^{2}$ & $l_{32}^{2}$ & $l_{33}^{2}$ & $l_{32} l_{31}$ & $l_{33} l_{32}$ & $l_{33} l_{31}$ \\
\hline 4 & $2 l_{21} l_{11}$ & $2 l_{12} l_{22}$ & $2 l_{13} l_{23}$ & $l_{11} l_{22}+l_{12} l_{21}$ & $l_{13} l_{32}+l_{12} l_{23}$ & $l_{13} l_{21}+l_{11} l_{23}$ \\
\hline 5 & $2 l_{31} l_{21}$ & $2 l_{32} l_{22}$ & $2 l_{33} l_{23}$ & $l_{31} l_{22}+l_{32} l_{21}$ & $l_{33} l_{22}+l_{32} l_{23}$ & $l_{33} l_{21}+l_{31} l_{23}$ \\
\hline 6 & $2 l_{31} l_{11}$ & $2 l_{32} l_{12}$ & $2 l_{33} l_{13}$ & $l_{31} l_{12}+l_{32} l_{11}$ & $l_{33} l_{12}+l_{32} l_{13}$ & $l_{33} l_{11}+l_{31} l_{13}$ \\
\hline
\end{tabular}

Fonte: LEKHNITSKII (1963).

Resultando:

$$
S_{11}^{\prime}=I_{11}^{4} S_{11}
$$

devido as demais parcelas que contribuem para $S_{11}^{\prime}$ serem nulas em (3.30).

Assim:

$$
S^{\prime}{ }_{11}=S_{11}
$$

De semelhante análise para os outros termos do tensor, conclui-se que:

$$
S_{15}=S_{16}=S_{25}=S_{26}=S_{35}=S_{36}=S_{45}=S_{46}=0
$$

Então, o tensor $S_{i j}$ para um material com simetria elástica em 1 (um) plano, torna-se: 


$$
S_{i j}=\left[\begin{array}{cccccc}
S_{11} & S_{12} & S_{13} & S_{14} & 0 & 0 \\
S_{21} & S_{22} & S_{23} & S_{24} & 0 & 0 \\
S_{31} & S_{32} & S_{33} & S_{34} & 0 & 0 \\
S_{41} & S_{42} & S_{43} & S_{44} & 0 & 0 \\
0 & 0 & 0 & 0 & S_{55} & S_{56} \\
0 & 0 & 0 & 0 & S_{65} & S_{66}
\end{array}\right]
$$

Com isto o tensor $S_{i j}$ passa a ter 13 (treze) componentes diferentes, sendo que apenas 11 (onze) são independentes, devido a dependência linear entre os termos $S_{55}$, $S_{56}$ e $S_{66}$. Semelhante análise pode ser realizada com o tensor $C_{i j}$.

\subsubsection{Material com Simetria Elástica em Três Planos (Material Ortótropo)}

Um sólido, referido a um sistema de coordenadas $x_{i}$, é denominado de material ortótropo, ou ortotrópico, quando possui três planos de simetria mutuamente perpendiculares, com eixos de simetria $x_{i}$.

Admitindo-se um plano desse sólido, referido a um sistema de coordenadas $x_{i}$, e procedendo-se agora uma rotação de $180^{\circ}$ em torno do eixo $x_{1}$, conforme Figura 3.3, tem-se, analogamente ao caso do material com um plano de simetria,

$$
S_{14}=S_{24}=S_{34}=S_{56}=0
$$

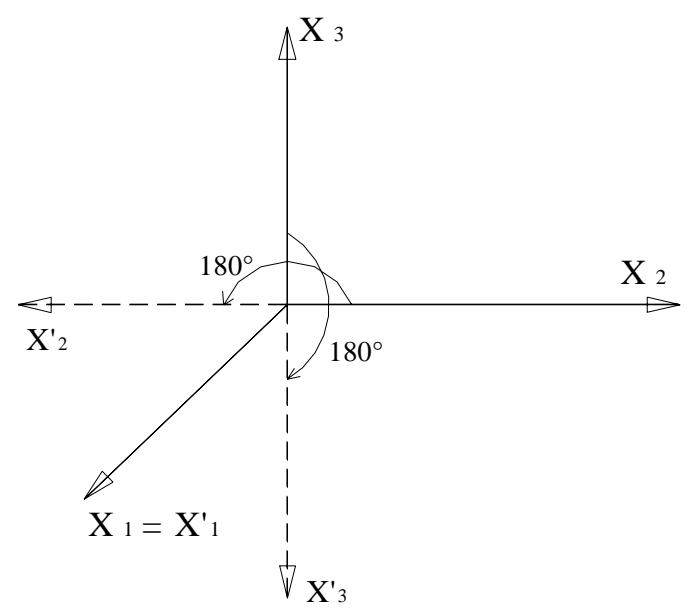

FIGURA 3.3 - Rotação de $180^{\circ}$ em torno do eixo $x_{1}$.

Efetuando-se semelhantes rotações nos eixos $x_{2}$ e $x_{3}$, uma de cada vez, tem-se: 


$$
\begin{aligned}
& S_{16}=S_{26}=S_{36}=S_{54}=0 \\
& S_{15}=S_{25}=S_{35}=S_{56}=0
\end{aligned}
$$

E o tensor $S_{i j}$ fica com a seguinte forma:

$$
S_{i j}=\left[\begin{array}{cccccc}
S_{11} & S_{12} & S_{13} & 0 & 0 & 0 \\
S_{21} & S_{22} & S_{23} & 0 & 0 & 0 \\
S_{31} & S_{32} & S_{33} & 0 & 0 & 0 \\
0 & 0 & 0 & S_{44} & 0 & 0 \\
0 & 0 & 0 & 0 & S_{55} & 0 \\
0 & 0 & 0 & 0 & 0 & S_{66}
\end{array}\right]
$$

Procedimento análogo pode ser aplicado ao tensor $C_{i j}$. Segundo LEKHNITSKII et al. (1968), quando 3 planos mutuamente perpendiculares de simetria elástica passam através de todos os pontos de um corpo homogêneo, as equações da lei de Hooke generalizada, referindo-se a um sistema de coordenadas $x, y, z$ serão:

$$
\begin{aligned}
& \varepsilon_{x}=S_{11} \sigma_{x}+S_{12} \sigma_{y}+S_{13} \sigma_{z} \\
& \varepsilon_{y}=S_{12} \sigma_{x}+S_{22} \sigma_{y}+S_{23} \sigma_{z} \\
& \varepsilon_{z}=S_{13} \sigma_{x}+S_{23} \sigma_{y}+S_{33} \sigma_{z} \\
& \gamma_{y z}=S_{44} \tau_{y z} \\
& \gamma_{x y}=S_{55} \tau_{x y} \\
& \gamma_{x z}=S_{66} \tau_{x z}
\end{aligned}
$$

Assim, para um material possuindo 3 (três) planos de simetria elástica, o número de constantes independentes será 9 (nove). Um elemento de um corpo ortotrópico na forma de um paralelepípedo retangular com lados paralelos aos planos de simetria elástica, submetido a ação de uma tensão normal aplicada em um de seus lados, permanece sem alteração de forma no processo de carregamento, ou seja, não ocorrerão distorções em seus planos devido a ausência dos coeficientes de influência mútua nas relações constitutivas. O sólido somente muda suas dimensões originais.

Dentre os tipos de simetria elástica, a ortotrópica é a mais presente em casos práticos de análise estrutural. De acordo com MELESH (1963), relações tensão- 
deformação ortotrópicas fornecem suficiente generalidade para descrever a maioria dos materiais estruturais.

\subsubsection{Material Transversalmente Isotrópico (Um Plano de Isotropia)}

Os materiais transversalmente isotrópicos são os que apresentam um plano de simetria física dentro do qual todas as direções são materialmente equivalentes (FUSCO, 1993).

Considerando-se um plano de um sólido referido a um sistema de coordenadas, conforme Figura 3.4, onde o plano $\boldsymbol{x}_{\mathbf{1}}-\boldsymbol{x}_{\mathbf{2}}$ é dito de isotropia, ou seja, todas as direções contidas nesse plano são elasticamente equivalentes e o eixo $x_{3}$ é o eixo de simetria elástica.

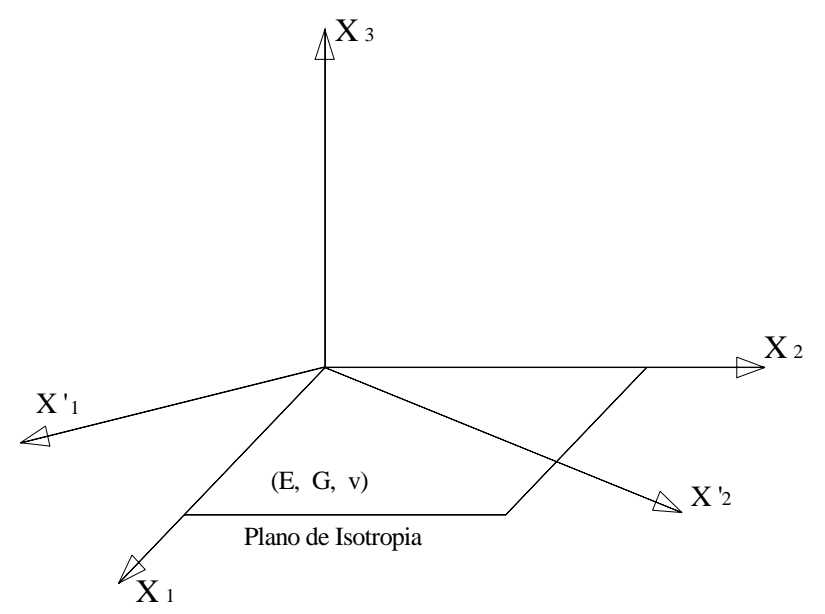

FIGURA 3.4 - Plano de isotropia - material transversalmente isotrópico.

Baseando-se nas equações dos itens anteriores, tem-se:

$$
S_{11}=S_{22} ; \quad S_{13}=S_{23} ; \quad S_{55}=S_{66} ; \quad 2\left(S_{11}-S_{22}\right)=S_{44}
$$

Assim, com a utilização da notação usual em engenharia, o tensor $S_{i j}$ torna-se: 


$$
S_{i j}=\left[\begin{array}{cccccc}
\frac{1}{E} & -\frac{v}{E} & -\frac{v^{\prime}}{E} & 0 & 0 & 0 \\
-\frac{v}{E} & \frac{1}{E} & -\frac{v^{\prime}}{E^{\prime}} & 0 & 0 & 0 \\
-\frac{v^{\prime}}{E} & -\frac{v^{\prime}}{E^{\prime}} & \frac{1}{E^{\prime}} & 0 & 0 & 0 \\
0 & 0 & 0 & \frac{1}{G} & 0 & 0 \\
0 & 0 & 0 & 0 & \frac{1}{G^{\prime}} & 0 \\
0 & 0 & 0 & 0 & 0 & \frac{1}{G^{\prime}}
\end{array}\right]
$$

onde $E$, $E$ ' são, respectivamente, o módulo de elasticidade no plano de isotropia e na direção normal a ele;

$v, v^{\prime}$ : coeficiente de Poisson no plano de isotropia e na direção normal a ele;

$G, G^{\prime}$ : módulo de elasticidade transversal no plano de isotropia e na direção normal a ele. E também:

$$
2\left(S_{11}-S_{12}\right)=S_{44}
$$

ou

$$
G=\frac{E}{2(1+v)}
$$

Portanto, apenas 5 (cinco) coeficientes de $S_{i j}$ são independentes. O módulo de elasticidade transversal $\boldsymbol{G}$ indica a isotropia no plano.

\subsubsection{Material Isotrópico}

Um corpo isotrópico é aquele em que todos os planos que passam por um ponto são isotrópicos, ou seja, todas as direções são elasticamente equivalentes e principais. Assim:

$$
E^{\prime}=E ; \quad G^{\prime}=G \quad \text { e } \quad v^{\prime}=v
$$

O tensor $S_{i j}$ torna-se: 


$$
S_{i j}=\left[\begin{array}{cccccc}
\frac{1}{E} & -\frac{v}{E} & -\frac{v}{E} & 0 & 0 & 0 \\
-\frac{v}{E} & \frac{1}{E} & -\frac{v}{E} & 0 & 0 & 0 \\
-\frac{v}{E} & -\frac{v}{E} & \frac{1}{E} & 0 & 0 & 0 \\
0 & 0 & 0 & \frac{1}{G} & 0 & 0 \\
0 & 0 & 0 & 0 & \frac{1}{G} & 0 \\
0 & 0 & 0 & 0 & 0 & \frac{1}{G}
\end{array}\right]
$$

Portanto, o tensor $S_{i j}$ passa a ter apenas 2 coeficientes independentes, ou seja, o módulo de elasticidade longitudinal $E$ e o coeficiente de Poisson $v$, sendo que o módulo de elasticidade transversal $G$ é definido como:

$$
G=\frac{E}{2(1+v)}
$$

Para corpos isotrópicos, quando da ocorrência de transformação de coordenadas, de um sistema $x, y, z$ para um $x^{\prime}, y^{\prime}, z^{\prime}$ as constantes elásticas preservam seus valores numéricos.

Segundo MUSKHELISHIVILI (1963), não existe na natureza corpos idealmente isotrópicos, porém, muitos materiais podem ser suficientemente considerados isotrópicos como, por exemplo, os metais, que são constituídos de microscópicos cristais anisotrópicos distribuídos aleatoriamente no material, conferindo assim, isotropia aos sólidos formados desse material.

No item seguinte, tendo-se já apresentado de forma geral algumas considerações sobre materiais anisotrópicos e suas relações constitutivas, apresenta-se então um breve comentário sobre a solução fundamental a ser utilizada no desenvolvimento da análise pelo MEC. 


\subsection{DESCRIÇÃO DA SOLUÇÃO FUNDAMENTAL} ANISOTRÓPICA UTILIZADA

A solução fundamental anisotrópica, apresentada por CRUSE \& SWEDLOW (1971), pode ser deduzida fazendo-se uso do formalismo de LEKHNITSKII (1963) e da teoria das funções complexas. Neste item, os principais passos dessa dedução são apresentados. O detalhamento completo da solução fundamental pode ser encontrado no Anexo 1.

Considere-se o tensor das tensões escrito em termos das funções de tensão de Airy $\left(F\left(x_{1}, x_{2}\right)\right)$ dadas por:

$$
\begin{aligned}
& \sigma_{11}=F_{,_{22}}+v \\
& \sigma_{22}=F,_{11}+v \\
& \sigma_{12}=-F_{,_{12}}
\end{aligned}
$$

onde $v$ é uma função potencial que, considerando o problema estático, tem valor nulo.

Essas funções satisfazem as equações de equilíbrio:

$$
\sigma_{i j},_{j}=0
$$

Expressando-se as deformações em termos das funções de Airy:

$$
\left\{\begin{array}{l}
\varepsilon_{1} \\
\varepsilon_{2} \\
\varepsilon_{6}
\end{array}\right\}=\left[\begin{array}{lll}
S_{11} & S_{12} & S_{16} \\
S_{12} & S_{22} & S_{26} \\
S_{16} & S_{26} & S_{66}
\end{array}\right] \cdot\left\{\begin{array}{c}
F_{, 22} \\
F_{, 11} \\
-F_{, 12}
\end{array}\right\}
$$

pode-se diferenciá-las como segue:

$$
\begin{aligned}
& \varepsilon_{1,22}=S_{11} \cdot F_{,_{2222}}+S_{12} \cdot F_{,_{1122}}-S_{16} \cdot F_{{ }_{1222}} \\
& \varepsilon_{2,11}=S_{12} \cdot F,_{2211}+S_{22} \cdot F_{,_{1111}}-S_{26} \cdot F_{r_{1211}} \\
& \varepsilon_{6,12}=S_{16} \cdot F,_{2212}+S_{26} \cdot F_{{ }_{1112}}-S_{66} \cdot F_{{ }_{1212}}
\end{aligned}
$$

Substituindo-se as expressões (3.53 - 3.55) nas equações de compatibilidade:

$$
\varepsilon_{1,22}+\varepsilon_{2,11}=\varepsilon_{6,12}
$$

obtém-se: 


$$
S_{11} F_{,_{2222}}-2 S_{16} F_{r_{1222}}+\left(2 S_{12}+S_{66}\right) F_{,_{1122}}-2 S_{26} F_{r_{1112}}+S_{22} F_{,_{1111}}=0
$$

que é a equação diferencial parcial de quarta ordem que modela um problema elástico anisotrópico nos moldes do formalismo de LEKHNITSKII (1963) e que tem como solução a função de tensão de Airy $\left(F\left(x_{1}, x_{2}\right)\right)$. Esta equação pode ser integrada na sua forma geral escrevendo-a simbolicamente com o uso de quatro operadores diferenciais lineares:

$$
\Delta_{1} \Delta_{2} \Delta_{3} \Delta_{4} F=0
$$

onde $\Delta$ designa a operação:

$$
\Delta_{k}=\frac{\partial}{\partial x_{2}}-\mu_{k} \frac{\partial}{\partial x_{1}} \quad(k=1,2,3,4)
$$

e $\mu_{k}$ são as raízes da equação característica (LEKHNITSKII et al., 1968):

$$
S_{11} \mu^{4}-2 S_{16} \mu^{3}+\left(2 S_{12}+S_{66}\right) \mu^{2}-2 S_{26} \mu+S_{22}=0
$$

Segundo LEKHNITSKII et al. (1968), as raízes da Equação (3.60) são sempre complexas ou imaginárias puras, não ocorrendo raízes reais para o caso de qualquer corpo elástico real com constantes $S_{11}, 2 S_{12}+S_{66}$ e $S_{22}$ finitas e diferentes de zero. Essas raízes complexas recebem a designação $\mu_{1}, \mu_{2}, \bar{\mu}_{1}$ e $\bar{\mu}_{2}$. Dois casos de combinações das raízes são possíveis, dependendo-se das relações entre as constantes elásticas:

$\left.1^{\circ}\right)$ As raízes são todas diferentes:

$$
\mu_{1}=\alpha+\beta \cdot i, \mu_{2}=\gamma+\delta \cdot i, \bar{\mu}_{1}=\alpha-\beta \cdot i, \bar{\mu}_{2}=\gamma-\delta \cdot i
$$

onde $\alpha, \beta, \gamma, \delta \in \mathrm{R}$ e $\beta>0$ e $\delta>0$.

$\left.2^{\circ}\right)$ As raízes são pares conjugados:

$$
\mu_{1}=\mu_{2}=\alpha+\beta \cdot i \text { e } \bar{\mu}_{1}=\bar{\mu}_{2}=\alpha-\beta \cdot i
$$

As raízes $\mu_{1}$ e $\mu_{2}$ são parâmetros complexos que podem ser considerados como números que caracterizam o grau de anisotropia em problemas planos. De acordo com seus valores, pode-se avaliar o quanto um dado corpo anisotrópico difere de um isotrópico, para o qual, $\mu_{1}=\mu_{2}=i$ e $\left|\mu_{1}\right|=\left|\mu_{2}\right|=1$. 
A partir da Equação (3.60) pode-se resolver o problema elástico evitando-se resolver diretamente a equação diferencial (3.57). Para tanto, procede-se a seguinte mudança de variável, onde o domínio $\Omega$ é mapeado num plano complexo:

$$
z_{k}=x_{1}+\mu_{k} x_{2} \quad(k=1,2)
$$

Para se obter as componentes de tensão em função das novas variáveis complexas, é preciso lembrar que a função de tensão é real e pode ser escrita como uma combinação linear de funções de tensão complexas:

$$
F\left(x_{1}, x_{2}\right)=2 \operatorname{Re}\left[F_{1}\left(z_{1}\right)+F_{2}\left(z_{2}\right)\right]
$$

onde $R e$ é a designação para a parte real de qualquer expressão complexa. Lembrandose ainda que as componentes de tensão são dadas de acordo com a função de Airy:

$$
\sigma_{11}=\frac{\partial^{2} F}{\partial x_{2}^{2}} ; \quad \sigma_{22}=\frac{\partial^{2} F}{\partial x_{1}^{2}} ; \quad \sigma_{12}=-\frac{\partial^{2} F}{\partial x_{1} \partial x_{2}}
$$

Segundo CRUSE \& SWEDLOW (1971), os deslocamentos fundamentais (solução fundamental de deslocamento) são dados individualmente por:

$$
U_{k i}\left(z^{\prime}, z\right)=2 \operatorname{Re}\left[q_{i 1} A_{k 1} \ln \left(z_{1}-z_{1}^{\prime}\right)+q_{i 2} A_{k 2} \ln \left(z_{2}-z_{2}^{\prime}\right)\right]
$$

As forças de superfície fundamentais são:

$$
T_{k i}\left(z^{\prime}, z\right)=2 \operatorname{Re}\left[\begin{array}{c}
\frac{1}{\left(z_{1}-z_{1}^{\prime}\right)} g_{i 1}\left(\mu_{1} \eta_{1}-\eta_{2}\right) A_{k 1}+ \\
\frac{1}{\left(z_{2}-z_{2}^{\prime}\right)} g_{i 2}\left(\mu_{2} \eta_{1}-\eta_{2}\right) A_{k 2}
\end{array}\right]
$$

onde:

$$
\left[g_{i j}\right]=\left[\begin{array}{cc}
\mu_{1} & \mu_{2} \\
-1 & -1
\end{array}\right]
$$

e $\eta_{k}$ são as componentes do vetor normal externo.

Nas soluções fundamentais apresentadas, $q_{i j}$ é a matriz de parâmetros complexos: 


$$
q_{i k}=\left[\begin{array}{c}
a_{11} \mu_{k}^{2}+a_{12}-a_{16} \mu_{k} \\
a_{12} \mu_{k}+a_{22} / \mu_{k}-a_{26}
\end{array}\right]
$$

e as constantes complexas $A_{i k}$ são obtidas da solução do sistema complexo:

$$
\left[\begin{array}{cccc}
1 & -1 & 1 & -1 \\
\mu_{1} & -\bar{\mu}_{1} & \mu_{2} & -\bar{\mu}_{1} \\
q_{11} & -\bar{q}_{11} & q_{12} & -\bar{q}_{12} \\
q_{21} & -\bar{q}_{21} & q_{22} & -\bar{q}_{22}
\end{array}\right] \cdot\left\{\begin{array}{l}
A_{j 1} \\
\bar{A}_{j 1} \\
A_{j 2} \\
\bar{A}_{j 2}
\end{array}\right\}=\left\{\begin{array}{c}
\delta_{j 2} / 2 \pi \cdot i \\
-\delta_{j 1} / 2 \pi \cdot i \\
0 \\
0
\end{array}\right\}
$$

\subsection{CONSIDERAÇÕES GERAIS SOBRE RESISTÊNCIA DOS MATERIAIS ANISOTRÓPICOS}

A análise de ruptura de estruturas constituídas de materiais anisotrópicos é um tema complexo e de grande importância devido ao elevado número de aplicações industriais desses materiais. Considerando-se materiais totalmente anisotrópicos, devese admitir que a ruptura seja condicionada tanto pelas tensões normais quanto pelas tensões de cisalhamento, uma vez que as rupturas podem ocorrer em virtude de diferentes conjuntos de tensões que agem sobre o elemento.

Em se tratando de materiais anisotrópicos constituídos de elementos com diferentes resistências, como materiais compósitos reforçados por fibras, regiões com resistência inferior a de regiões vizinhas exercerão maior influência na resistência global do compósito do que regiões com alta resistência, uma vez que poderá ocorrer ruptura daquela antes desta.

De acordo com HYER (1997), para compósitos poliméricos reforçados com fibras, devido a direção das fibras ser relativamente mais resistente do que outras direções, a ruptura é uma função da direção de aplicação da carga. Para causar ruptura na direção das fibras é preciso uma maior tensão do que para causar a ruptura na direção perpendicular a elas. A ruptura à tração na direção das fibras é controlada pela resistência destas, enquanto a ruptura à tração perpendicular às fibras é controlada pela resistência da aderência entre a fibra e a matriz e pela resistência da própria matriz.

Porém, no caso de solicitações mais complexas, como solicitações orientadas segundo um ângulo qualquer em relação às fibras, a dificuldade de se encontrar qual é a 
tensão que controlará a ruptura é muito maior. Assim, procurando-se levar em consideração tais fenômenos de ruptura nos materiais compósitos, estabeleceram-se os chamados mecanismos ou modos de ruptura para materiais compósitos reforçados por fibras.

A identificação de tais modos de ruptura é feita por meio de uma análise micromecânica, que leva em consideração o fato de que os elementos constituintes dos compósitos (fibra e matriz) têm, em geral, características de resistência muito diferentes um dos outros. Além disso, a interface entre esses dois elementos básicos apresenta comportamento também diverso dos da fibra e da matriz. Assim, identificam-se de modo geral três modos de ruptura: ruptura da matriz, ruptura da fibra e ruptura da interface fibra-matriz.

Os modos básicos de ruptura são considerados, do ponto de vista da resistência global do compósito, como sendo apenas iniciadores da ruptura global. Para caracterizar a ruptura de um elemento estrutural laminado é necessário ainda caracterizar a ruptura da lâmina e do conjunto de lâminas. Uma tal caracterização se faz não mais pela micromecânica mas pela macromecânica ou análise estrutural (MAGAGNIN, 1996).

A caracterização da ruptura da lâmina se dá com base nos conhecidos critérios de ruptura e é uma caracterização macroscópica. Para a análise do conjunto de lâminas, quando se visa uma análise mais apurada, geralmente são realizadas também considerações sobre delaminação e deslizamento entre as lâminas. Porém, no contexto do presente trabalho, onde inicialmente pretende-se considerar, na análise viscoplástica, as estruturas laminadas compósitas com sendo homogêneas e anisotrópicas, e depois, na continuidade da pesquisa, considerar separadamente as fibras de reforço imersas em um meio homogêneo, não são realizadas investigações visando-se o entendimento das particularidades (como exemplo, delaminação) dos conjuntos de lâminas. Assim, na continuidade deste item, são considerados os principais critérios de ruptura empregados na análise macroscópica de resistência dos materiais anisotrópicos.

\subsubsection{Critérios de ruptura para materiais anisotrópicos e compostos}

O principal objetivo de um critério de resistência é fornecer parâmetros para se poder estimar rapidamente quando uma estrutura, constituída de determinado material e submetida a complexas situações de carregamento, entrará em ruptura. Muitos dos 
critérios de ruptura para compósitos anisotrópicos são baseados na generalização de critérios, previamente desenvolvidos, para materiais isotrópicos. Por isso, esses critérios também fazem uso do conceito de uma superfície de ruptura representada no espaço de tensões principais.

Segundo MAGAGNIN (1996), os critérios de resistência para materiais compósitos podem ser classificados como critérios predominantemente de tensão, predominantemente de deformação e critérios quadráticos interativos. O primeiro corresponde ao critério de Máxima Tensão e o segundo ao de Máxima Deformação.

O critério da Máxima Tensão para lâminas ortotrópicas, de acordo com GIBSON (1995), foi apresentado em 1920 por Jenkins ${ }^{1}$ como uma extensão da teoria de Rankine para materiais isotrópicos. Este critério prediz a ruptura quando qualquer componente de tensão principal atuante exceder uma tensão de referência especificada. Deste modo, considerando-se o estado plano de tensão, para se evitar a ruptura as seguintes desigualdades precisam ser satisfeitas:

$$
\sigma_{1} \leq X_{t} ; \quad \sigma_{2} \leq Y_{t} ; \quad \tau_{12} \leq T_{x y}
$$

onde $\sigma_{1}, \sigma_{2}$ são as componentes de tensão normais, $\tau_{12}$ a componente de tensão de cisalhamento; $X_{t}$ e $Y_{t}$ são as resistências normais da lâmina à tração (ou compressão) nas direções $x$ e $y$ respectivamente e $T_{x y}$ é a resistência ao cisalhamento no plano $x y$.

A superfície de ruptura para o critério da Máxima Tensão no espaço $\sigma_{1}-\sigma_{2}$ é um retângulo (Figura 3.5). A superfície de ruptura é independente da tensão de cisalhamento $\tau_{12}$ e este critério não considera a possibilidade de interação entre as componentes de tensões, ou seja, o valor de uma determinada resistência limite é independente da presença, ou não, de outra componente de tensão.

O critério da Máxima Deformação é na sua forma geral similar ao critério da Máxima Tensão. Segundo GIBSON (1995), foi proposto por Waddoups² em 1967 como uma extensão da teoria da máxima deformação normal para materiais isotrópicos. Este

\footnotetext{
${ }^{1}$ Jenkins, C. F., Report on materials of constructions used in aircraft and aircraft engines, Great Britain Aeronautical Research Committee, 1920.

${ }^{2}$ Waddoups, M. E., Advanced composite material mechanics for the design and stress analysis, General Dynamics, Fort Worth Division Report FZM-4763, Fort Worth, TX (1967)
} 
critério prediz a ruptura quando qualquer componente de deformação principal atuante exceder uma deformação última especificada:

$$
\varepsilon_{1} \leq X_{\varepsilon t} ; \quad \quad \varepsilon_{2} \leq Y_{\varepsilon t} ; \quad\left|\gamma_{12}\right| \leq T_{\varepsilon_{12}}
$$

onde $\varepsilon_{1}, \varepsilon_{2}$ são as componentes de deformação à tração normal nas direções $x$ e $y$ respectivamente e $\gamma_{12}$ é a componente de deformação por cisalhamento no plano $x y$; $X_{s t}$ e $Y_{s t}$ são as resistências à deformação da lâmina à tração (ou compressão) nas direções $x$ e $y$ respectivamente e $T_{\varepsilon 12}$ é a resistência à deformação por cisalhamento no plano xy. Como no critério da Máxima Tensão, assume-se também, que a ruptura ao cisalhamento é independente do sinal da deformação cisalhante $\gamma_{12}$.

A superfície de ruptura para o critério da Máxima Deformação no espaço $\varepsilon_{1}-\varepsilon_{2}$ é um retângulo similar ao do critério da Máxima Tensão no espaço $\sigma_{1}-\sigma_{2}$. Entretanto, no espaço $\sigma_{1}-\sigma_{2}$, a superfície do critério da Máxima Deformação é um paralelogramo distorcido, cuja forma é dependente da relação tensão-deformação da lâmina, como mostrado na Figura 3.5.

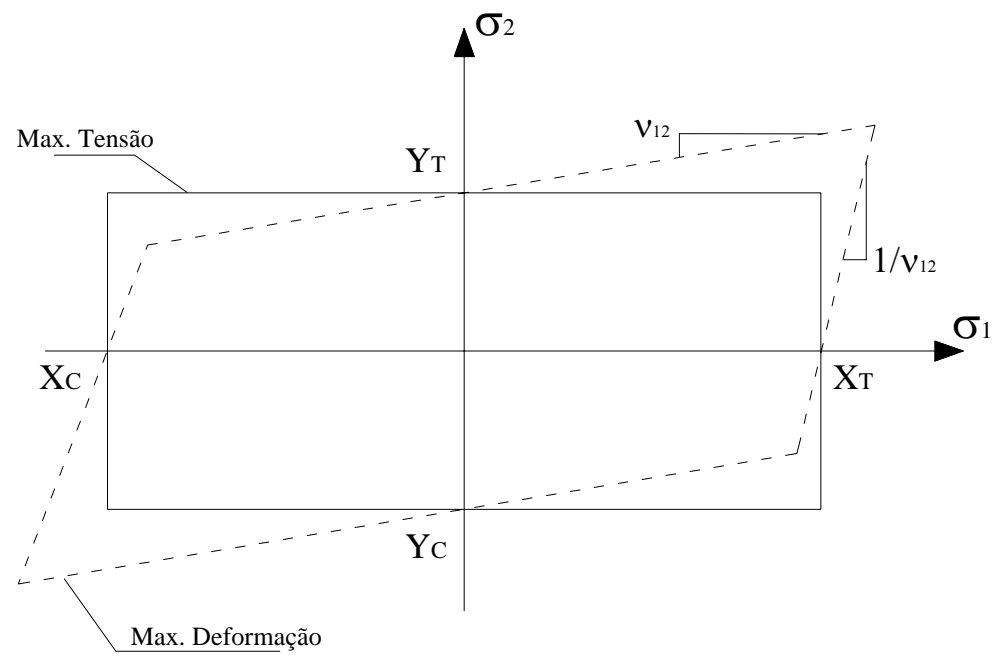

FIGURA 3.5 - Superfícies dos critérios de Máxima Deformação e de Máxima Tensão.

Os critérios interativos são os polinômios de tensões que diferem dos dois primeiros critérios devido ao fato de incluírem termos quadráticos que consideram a interação entre as componentes de tensões, originando superfícies de rupturas elípticas. Os critérios mais difundidos na literatura são o de HILL (1948), o de Tsai-Hill, o de 
HOFFMAN (1967), os de Hashin e por fim, o critério de TSAI \& WU (1971), que é, entre estes, o mais geral para análise de materiais anisotrópicos.

De acordo com GIBSON (1995), em 1948, Hill sugeriu que o critério de Von Mises poderia ser modificado para se poder incluir os efeitos do comportamento anisotrópico induzido em metais devido a ocorrência de grandes deformações plásticas. Para um estado geral 3D de tensões ao longo dos eixos principais de um material, a superfície de ruptura para o critério de HILL (1948) no espaço $\sigma_{1}-\sigma_{2}-\sigma_{3}$ é descrita pela equação:

$$
\begin{aligned}
A\left(\sigma_{2}-\sigma_{3}\right)^{2}+B\left(\sigma_{3}-\sigma_{1}\right)^{2}+C\left(\sigma_{1}-\sigma_{2}\right)^{2} & +2 \cdot D \cdot \tau_{23}^{2}+ \\
& +2 \cdot E \cdot \tau_{31}^{2}+2 \cdot F \cdot \tau_{12}^{2}=1
\end{aligned}
$$

onde $A, B, C, D, E$, e $F$ são constantes dependentes das resistências ao escoamento determinadas a partir de ensaios uniaxiais e de cisalhamento simples.

No critério de HILL (1948), as resistências ao escoamento na tração e na compressão são assumidas iguais. Considerando-se a Equação (3.75) e, isoladamente, ensaios uniaxiais nas direções principais 1, 2 e 3 e também, testes de cisalhamento simples nos planos 23, 31 e 12, pode-se obter as constantes:

$$
\begin{aligned}
& 2 \cdot A=\frac{1}{Y_{2}^{2}}+\frac{1}{Y_{3}^{2}}-\frac{1}{Y_{1}^{2}} \\
& 2 \cdot B=\frac{1}{Y_{3}^{2}}+\frac{1}{Y_{1}^{2}}-\frac{1}{Y_{2}^{2}} \\
& 2 \cdot C=\frac{1}{Y_{1}^{2}}+\frac{1}{Y_{2}^{2}}-\frac{1}{Y_{3}^{2}} \\
& 2 \cdot D=\frac{1}{Y_{23}^{2}} \\
& 2 \cdot E=\frac{1}{Y_{31}^{2}} \\
& 2 \cdot F=\frac{1}{Y_{12}^{2}}
\end{aligned}
$$

onde $Y_{1}, Y_{2}, Y_{3}$ são resistências ao escoamento uniaxiais ao longo das direções 1, 2 e 3 respectivamente e $Y_{12}, Y_{23}, Y_{31}$ são resistências ao escoamento ao cisalhamento associadas aos planos 12, 23 e 31 respectivamente. Maiores detalhes sobre o critério de 
HILL (1948) são apresentados nos trabalhos de SHIH \& LEE (1978), de FEENSTRA \& de BORST (1990) e de KHAROUF et al. (2003).

TSAI (1968) propôs uma extensão do critério de escoamento de HILL (1948), para a análise de lâminas ortotrópicas, admitindo que o critério de ruptura de um composto de fibras unidirecionais tem a mesma forma matemática do critério de escoamento de um material idealmente plástico, como o de HILL (1948), onde a ruptura é associada à tensão de cisalhamento e independente da tensão hidrostática. Assim, esse novo critério ficou conhecido como o critério de Tsai-Hill.

Assumindo-se uma lâmina ortotrópica, no estado plano de tensão ( $\sigma_{3}=\tau_{31}=\tau_{23}=0$ ), com a direção principal 1 sendo a direção da fibra de reforço, e ainda, admitindo-se que as resistências ao escoamento da expressão do critério de HILL (1948) são substituídas pelas resistências ao escoamento da lâmina, assim denominadas $Y_{1}=s_{L}, Y_{2}=Y_{3}=s_{T}$ e $Y_{12}=s_{L T}$, pode-se obter a partir das Equações $(3.76-3.81)$ a expressão para o critério de ruptura de Tsai-Hill:

$$
\frac{\sigma_{1}^{2}}{s_{L}^{2}}-\frac{\sigma_{1} \cdot \sigma_{2}}{s_{L}^{2}}+\frac{\sigma_{2}^{2}}{s_{T}^{2}}+\frac{\tau_{12}^{2}}{s_{L T}^{2}}=1
$$

A superfície gerada pela expressão do critério de Tsai-Hill no espaço de tensões principais é uma elipse simétrica, devido a hipótese de iguais resistências ao escoamento na tração e na compressão, como se mostra na Figura 3.6:

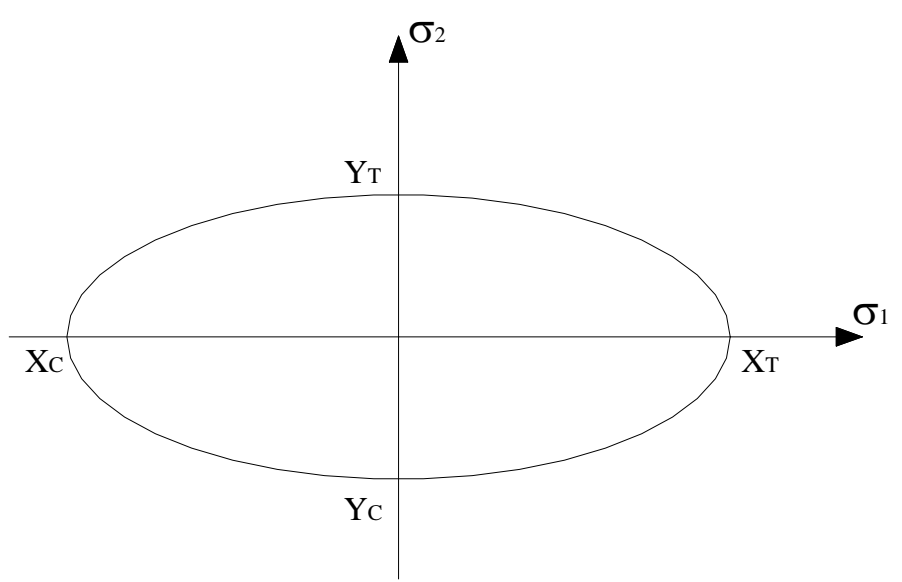

FIGURA 3.6 - Superfície de ruptura do critério de Tsai-Hill.

No entanto, a equação do critério de Tsai-Hill pode também ser utilizada quando as resistências à tração e à compressão são diferentes, embora tal procedimento 
seja inconsistente com as formulações originais dos critérios de Von Mises e de HILL (1948), simplesmente utilizando-se valores de $s_{L}$ e de $s_{T}$ apropriados para cada quadrante do espaço de tensões principais (TSAI, 1968).

O critério de resistência de HOFFMAN (1967) propõe uma condição de ruptura, para materiais frágeis ortotrópicos, que leva em conta diferentes resistências na tração e na compressão através da presença de termos lineares nas tensões normais $\sigma_{1}$, $\sigma_{2}$ e $\sigma_{3}$, diferenciando-se então, dos critérios de HILL (1948) e de Tsai-Hill.

Segundo HOFFMAN (1967), sob baixas temperaturas e/ou sob carregamento de curta duração, as fibras e a matriz têm comportamento frágil e assim, o material pode sofrer uma ruptura também frágil. Sendo a fragilidade caracterizada por grandes diferenças entre as resistências à compressão e à tração, o critério considera nove parâmetros do material e a resistências à compressão e à tração em várias direções. A expressão do critério é representada na Equação (3.83):

$$
\begin{array}{r}
C_{1}\left(\sigma_{y}-\sigma_{z}\right)^{2}+C_{2}\left(\sigma_{z}-\sigma_{x}\right)^{2}+C_{3}\left(\sigma_{x}-\sigma_{y}\right)^{2}+C_{4} \sigma_{x}+C_{5} \sigma_{y}+ \\
+C_{6} \sigma_{z}+C_{7} \tau_{y z}^{2}+C_{8} \tau_{z x}^{2}+C_{9} \tau_{x y}^{2}=1
\end{array}
$$

onde os parâmetros $C_{i}$ são dependentes do tipo de material e das resistências à compressão e à tração nas direções dos eixos coordenados e também, das resistências ao cisalhamento puro nos planos coordenados.

Para o estado plano de tensão, com as fibras na direção $x$, a condição de ruptura torna-se (HOFFMAN, 1967):

$$
\frac{\sigma_{x}^{2}-\sigma_{x} \sigma_{y}}{F_{c x} F_{t x}}+\frac{\sigma_{y}^{2}}{F_{c y} F_{t y}}+\frac{F_{c x}-F_{t x}}{F_{c x} F_{t x}} \sigma_{x}+\frac{F_{c y}-F_{t y}}{F_{c y} F_{t y}} \sigma_{y}+\frac{\tau_{x y}^{2}}{F_{s x y}^{2}}=1
$$

onde $F_{c i}, F_{t i}, F_{s i j}$ são respectivamente resistências à compressão, à tração e ao cisalhamento puro. A expressão de ruptura (3.84) é geometricamente representada no espaço de tensões principais por uma superfície em forma de elipse (Figura 3.7). 


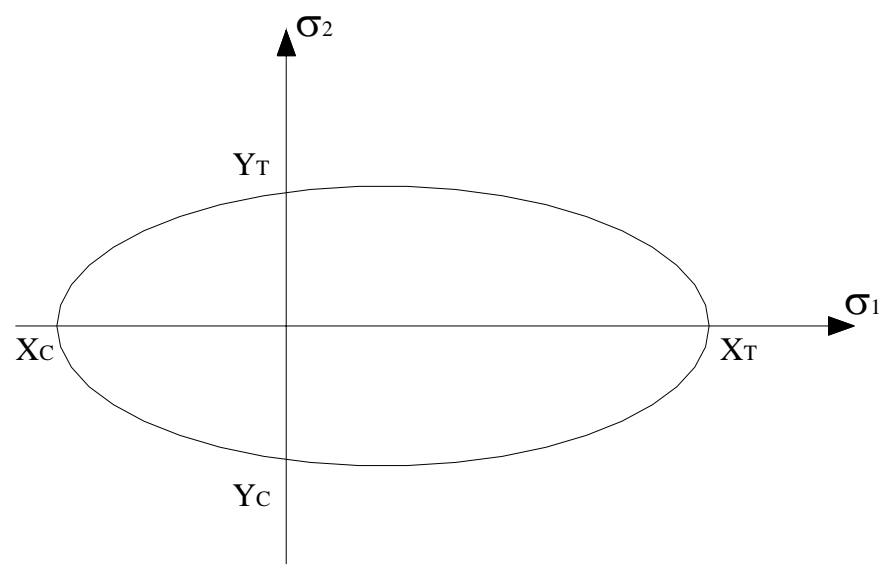

FIGURA 3.7 - Superfície de ruptura do critério de HOFFMAN (1967).

Existem dois critérios de ruptura, para materiais compostos fibrosos unidirecionais, que estão atribuídos a Hashin. A consideração de mecanismos de rupturas no desenvolvimento de ambos critérios é a principal diferença entre esses e os critérios já apresentados.

No primeiro critério, HASHIN \& ROTEM (1973) propõe dois mecanismos de ruptura para o composto: um baseado na ruptura da fibra e o outro baseado na ruptura da matriz. O primeiro é governado pela tensão longitudinal referente à orientação da fibra, e o segundo, é governado pelas tensões transversais à fibra.

A importância do critério de HASHIN \& ROTEM (1973) está no fato de que os autores iniciaram um diferente meio de se desenvolver critérios aproximados de resistência para materiais compósitos. Primeiro, eles reconheceram os modos de ruptura e então, reconheceram as variáveis associadas com esses modos e propuseram a interação entre elas. A idéia apresentada pelos autores se mostra bastante adequada para certos tipos de materiais, embora, nem todos os modos de ruptura que aparecem nesses tipos de materiais possam ser considerados pela aproximação proposta por eles.

Assim, HASHIN (1980) reexaminou a proposta do primeiro critério e estabeleceu algumas modificações. Com relação a ruptura na direção das fibras, a diferença está na consideração da contribuição da tensão de cisalhamento $\sigma_{12}$ na ruptura à tração, procurando-se assim, adequar um pouco mais o critério ao comportamento anisotrópico desses materiais. A segunda diferença está no modo de ruptura à compressão da matriz, por meio da hipótese de que um estado quaseisotrópico de tensão produzirá a ruptura num valor muito maior do que o da resistência transversal às fibras, diferindo-se assim, da proposta de HASHIN \& ROTEM (1973). 
Todos os critérios de resistência apresentados, até este ponto, são casos especiais de um critério mais geral de interação quadrática desenvolvido por Tsai e por Wu em 1971 e que, devido sua generalidade, tem sido muito usado em diversas análises envolvendo materiais anisotrópicos naturais ou industriais, podendo-se citar BRÜNIG (1995), CLOUSTON \& LAM (2001) e KOLAKOWSKI (2003).

Os autores propuseram uma melhor e ao mesmo tempo versão simplificada da teoria de ruptura, representada por tensores polinomiais, sugerida primeiramente por Gol'denblat e Koprov ${ }^{3}$ em 1965. No critério de ruptura geral de TSAI \& WU (1971), a superfície de ruptura no espaço de tensões principais é descrita pela expressão tensorial polinomial:

$$
F_{i} \sigma_{i}+F_{i j} \sigma_{i} \sigma_{j}=1 \quad(\mathrm{i}, \mathrm{j}=1,2, \ldots, 6)
$$

que expandida, adquire a forma:

$$
\begin{aligned}
& F_{1} \sigma_{1}+F_{2} \sigma_{2}+F_{3} \sigma_{3}+F_{4} \sigma_{4}+F_{5} \sigma_{5}+F_{6} \sigma_{6}+ \\
& +F_{11} \sigma_{1}^{2}+2 F_{12} \sigma_{1} \sigma_{2}+2 F_{13} \sigma_{1} \sigma_{3}+2 F_{14} \sigma_{1} \sigma_{4}+2 F_{15} \sigma_{1} \sigma_{5}+2 F_{16} \sigma_{1} \sigma_{6}+ \\
& +F_{22} \sigma_{2}^{2}+2 F_{23} \sigma_{2} \sigma_{3}+2 F_{24} \sigma_{2} \sigma_{4}+2 F_{25} \sigma_{2} \sigma_{5}+2 F_{26} \sigma_{2} \sigma_{6}+ \\
& +F_{33} \sigma_{3}^{2}+2 F_{34} \sigma_{3} \sigma_{4}+2 F_{35} \sigma_{3} \sigma_{5}+2 F_{36} \sigma_{3} \sigma_{6}+F_{44} \sigma_{4}^{2}+ \\
& +2 F_{45} \sigma_{4} \sigma_{5}+2 F_{46} \sigma_{4} \sigma_{6}+F_{55} \sigma_{5}^{2}+2 F_{56} \sigma_{5} \sigma_{6}+F_{66} \sigma_{6}^{2}=1
\end{aligned}
$$

Os termos lineares $\sigma_{i}$ consideram tensões que descrevem rupturas induzidas por diferenças entre tensões positivas e negativas. Os termos quadráticos $\sigma_{i} \sigma_{j}$ definem um elipsóide no espaço de tensões principais. Os valores dos termos de interação $F_{i j}$ são limitados pela desigualdade (HYER, 1997):

$$
\frac{1}{2} F_{i i} F_{j j}-F_{i j}^{2} \geq 0
$$

condição essa que, geometricamente, assegura que a superfície de ruptura intercepte cada eixo de tensão e que sua forma seja de um elipsóide (Figura 3.8).

\footnotetext{
${ }^{3}$ Gol'denblat, I. and Kopnov, V. A., Strength of glass reinforced plastics in the complex stress state, Mekhanika Polimerov, 1, $70-78$ (1965). English translation: Polymer Mechanics, 1, 54-60 (1966).
} 
Para o caso plano de tensão, com $\sigma_{3}=\sigma_{33}=0, \quad \sigma_{4}=\sigma_{23}=0 \quad$ e $\sigma_{5}=\sigma_{31}=0$, a Equação geral (3.86) torna-se:

$$
\begin{gathered}
F_{11} \sigma_{1}^{2}+F_{22} \sigma_{2}^{2}+F_{66} \sigma_{6}^{2}+F_{1} \sigma_{1}+F_{2} \sigma_{2}+2 F_{12} \sigma_{1} \sigma_{2}+ \\
+2 F_{16} \sigma_{1} \sigma_{6}+2 F_{26} \sigma_{2} \sigma_{6}=1
\end{gathered}
$$

onde a tensão $\sigma_{6}$ é a tensão de cisalhamento $\tau_{12}$.

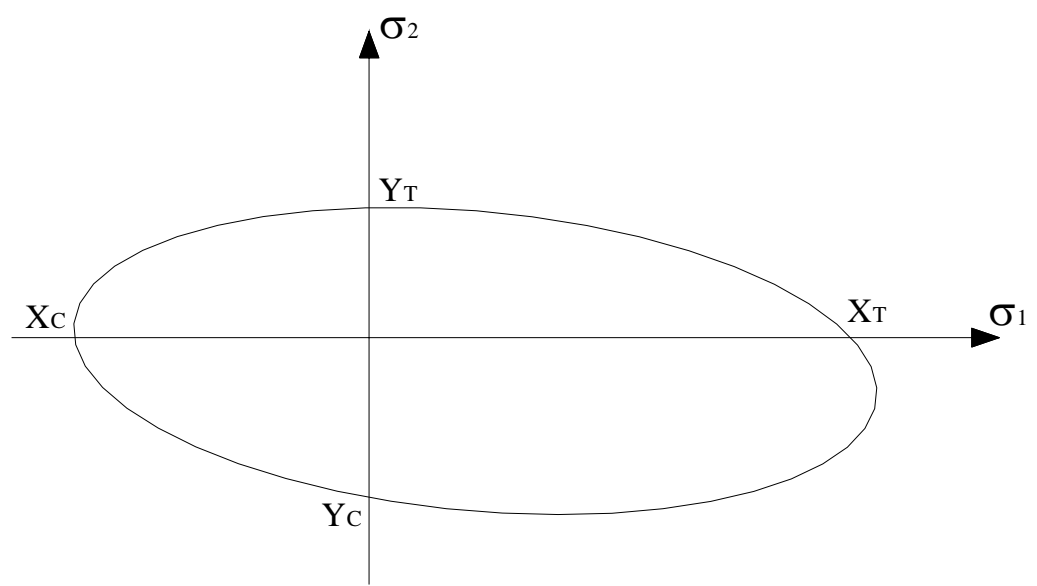

FIGURA 3.8 - Superfície de ruptura do critério de TSAI \& WU (1971).

Segundo TSAI \& WU (1971), os tensores de resistência $F_{i}$ e $F_{i j}$ possuem as mesmas propriedades de simetria elástica que os tensores constitutivos de rigidez $\left(C_{i j}\right)$ e de compliância $\left(S_{i j}\right)$ possuem, inclusive o número de elementos independentes e diferentes de zero. Os autores afirmam também, que a maioria dos critérios existentes são limitados a materiais ortotrópicos, enquanto que o presente critério pode ser aplicado para materiais anisotrópicos gerais, permitindo inclusive, transformações dos tensores de resistência $F_{i}$ e $F_{i j}$ para outros sistemas de coordenadas.

Dependendo do material presente na análise, os tensores de resistência $F_{i}$ e $F_{i j}$ podem ser simplificados, diminuindo-se a quantidade de termos de interação $F_{i j}$. Considerando-se um material ortotrópico plano, por exemplo, os termos $F_{16}$ e $F_{26}$ presentes na Equação (3.88) desaparecem pois são específicos para materiais anisotrópicos, semelhantes aos coeficientes de influência mútua $\left(\eta_{i j, i}\right)$, item 3.2.1.

Os elementos dos tensores de resistência $F_{i}$ e $F_{i j}$ são determinados em laboratório através de ensaios de tração e de compressão simples, bem como, de cisalhamento puro. Dessa forma, os parâmetros de resistência podem ser escritos: 


$$
\begin{array}{lll}
F_{1}=\frac{1}{X}-\frac{1}{X^{\prime}} ; & F_{2}=\frac{1}{Y}-\frac{1}{Y^{\prime}} ; & F_{3}=\frac{1}{Z}-\frac{1}{Z^{\prime}} \\
F_{4}=\frac{1}{Q}-\frac{1}{Q^{\prime}} ; & F_{5}=\frac{1}{R}-\frac{1}{R^{\prime}} ; & F_{6}=\frac{1}{S}-\frac{1}{S^{\prime}} \\
F_{11}=\frac{1}{X \cdot X^{\prime}} ; & F_{22}=\frac{1}{Y \cdot Y^{\prime}} ; & F_{33}=\frac{1}{Z \cdot Z^{\prime}} \\
F_{44}=\frac{1}{Q \cdot Q^{\prime}} ; & F_{55}=\frac{1}{R \cdot R^{\prime}} ; & F_{66}=\frac{1}{S \cdot S^{\prime}}
\end{array}
$$

onde $X$ e $X^{\prime} ; Y$ e $Y^{\prime} ; Z$ e $Z^{\prime}$ são, respectivamente, as resistências à tração e à compressão nas direções coordenadas 1, 2 e 3; $Q$ e $Q^{\prime} ; R$ e $R^{\prime}$; $S$ e $S^{\prime}$ são, respectivamente, as resistências positiva e negativa ao cisalhamento puro nos planos 2-3, 3-1 e 1-2.

Para a determinação experimental dos termos $F_{i j}$ (com $\mathrm{i} \neq \mathrm{j}$ ) não são suficientes ensaios uniaxiais ou de cisalhamento simples. Sua determinação requer combinações de estados de tensões, sendo possível então, infinitas combinações e formas diferentes de ensaios. Por exemplo, com a realização de um ensaio biaxial de tensão, tal que:

$$
\sigma_{1}=\sigma_{2}=P, \quad \sigma_{3}=\sigma_{4}=\sigma_{5}=\sigma_{6}=0
$$

e, substituindo-se esse estado combinado na Equação (3.86), obtém-se:

$$
P^{2}\left(F_{11}+F_{22}+2 F_{12}\right)+P\left(F_{1}+F_{2}\right)=1
$$

que, sendo resolvida para $F_{12}$, origina:

$$
F_{12}=\frac{1}{2 P^{2}}\left[1-P\left(\frac{1}{X}-\frac{1}{X^{\prime}}+\frac{1}{Y}-\frac{1}{Y^{\prime}}\right)-P^{2}\left(\frac{1}{X \cdot X^{\prime}}+\frac{1}{Y \cdot Y^{\prime}}\right)\right]
$$

Para um material anisotrópico, a componente de resistência $F_{16}$ pode ser determinada através de um ensaio de tração com cisalhamento, isto é:

$$
\sigma_{1}=\sigma_{6}=T, \quad \sigma_{2}=\sigma_{3}=\sigma_{4}=\sigma_{5}=0
$$

Substituindo-se esse estado combinado na Equação (3.86), obtém-se:

$$
F_{16}=\frac{1}{2 T^{2}}\left[1-T\left(\frac{1}{X}-\frac{1}{X^{\prime}}+\frac{1}{S}-\frac{1}{S^{\prime}}\right)-T^{2}\left(\frac{1}{X \cdot X^{\prime}}+\frac{1}{S \cdot S^{\prime}}\right)\right]
$$


Uma outra forma de se obter tais propriedades de resistência é por meio de transformações das propriedades de resistências entre sistemas de coordenadas, valendo-se de operações idênticas as que podem ser efetuadas com os tensores constitutivos $C_{i j}$ e $S_{i j}$. Sendo conhecidas as propriedades para um sistema local de coordenadas de uma lâmina ortotrópica reforçada por fibras e assumindo-se que esta lâmina esteja rotacionada por um determinado ângulo em relação a um sistema global de coordenadas, pode-se expressar as resistências de cada lâmina para o sistema global do compósito por meio de transformações trigonométricas, surgindo-se assim, os termos $F_{16}$ e $F_{26}$ no tensor de resistência $F_{i j}$ do compósito. Assim, pode-se compor propriedades de resistência para materiais anisotrópicos industriais pela superposição de um conjunto de lâminas ortotrópicas.

Tendo-se realizado uma fundamentação teórica geral sobre critérios de rupturas para materiais anisotrópicos, finaliza-se então, este capítulo sobre considerações gerais e de resistência sobre meios anisotrópicos ressaltando-se que o critério de resistência de TSAI \& WU (1971), devido sua generalidade, é o critério empregado nas análises físicas não lineares deste trabalho, incluindo-se sua degeneração para o caso isotrópico. 


\section{FORMULAÇÃO DO MEF PARA O PROBLEMA VISCOPLÁSTICO ANISOTRÓPICO}

Neste capítulo, apresenta-se a formulação do Método dos Elementos Finitos (MEF) para a análise de problemas viscoplásticos em meios anisotrópicos, abordandose também, dessa forma, os problemas elásticos, viscoelásticos e elastoplásticos.

\subsection{FORMULAÇÃO INTEGRAL PARA A ABORDAGEM DO PROBLEMA}

Para a formulação do problema via MEF, busca-se inicialmente a representação integral do equilíbrio estático de um corpo, partindo-se da equação de equilíbrio estático de uma porção infinitesimal do sólido:

$$
\sigma_{i j, j}+b_{i}=0
$$

onde $b_{i}$ representa as componentes das forças de volume. Pode-se ponderar o erro produzido pela Equação (4.1), quando a solução exata é substituída por uma aproximada, utilizando-se como função ponderadora a função de deslocamentos virtuais $\delta u_{i}$. Assim, a equação de ponderação sobre todo o domínio $\Omega$ pode ser escrita como:

$$
\int_{\Omega} \delta u_{i}\left(\sigma_{i j, j}+b_{i}\right) d \Omega=0=\int_{\Omega} R_{i} \cdot \delta u_{i} d \Omega
$$


Integrando-se por partes o primeiro termo da Equação (4.2), obtém-se:

$$
\int_{\Gamma} \delta u_{i} \sigma_{i j} \eta_{j} d \Gamma-\int_{\Omega} \delta u_{i, j} \sigma_{i j} d \Omega+\int_{\Omega} \delta u_{i} b_{i} d \Omega=0
$$

onde $\Gamma$ que define o contorno do corpo e $\eta_{j}$ a componente $j$ do versor normal à superfície. Sabendo-se que $\sigma_{i j} \eta_{j}=p_{i}$ e que $\delta u_{i, j} \sigma_{i j}=\delta \varepsilon_{i j} \sigma_{i j}$, onde $\delta \varepsilon_{i j}$ são as componentes de deformação virtual, a Equação (4.3) torna-se:

$$
\int_{\Gamma} \delta u_{i} p_{i} d \Gamma-\int_{\Omega} \delta \varepsilon_{i j} \sigma_{i j} d \Omega+\int_{\Omega} \delta u_{i} b_{i} d \Omega=0
$$

que é a expressão do princípio dos trabalhos virtuais para o problema estático. A primeira e a terceira integrais representam, respectivamente, o trabalho das forças de superfície e das volumétricas. A segunda integral refere-se ao trabalho das forças internas e dá origem à matriz de rigidez.

A Equação (4.4) é o ponto de partida para a obtenção da representação integral do MEF para os problemas anisotrópicos planos abordados neste trabalho. Inicialmente, para se encontrar a formulação do problema elástico, é preciso substituir a relação constitutiva (3.4) na Equação (4.4):

$$
\int_{\Gamma} \delta u_{i} p_{i} d \Gamma-\int_{\Omega} \delta \varepsilon_{i j} C_{i j k l} \varepsilon_{k l} d \Omega+\int_{\Omega} \delta u_{i} b_{i} d \Omega=0
$$

A Equação (4.5) é a representação integral para o problema elástico do MEF. Se no problema abordado as forças volumétricas forem desconsideradas, a última integral presente na Equação (4.5) desaparece.

Para se descrever a formulação do problema viscoelástico, é importante tecer alguns comentários sobre viscosidade e sobre a forma como ela é abordada no presente trabalho.

Na análise estrutural, um problema essencial é a modelagem do comportamento do material constituinte do meio. Em geral, o comportamento físico dos materiais é influenciado por vários parâmetros, tais como, tempo, temperatura, condições de carregamento, etc. Com isso, procura-se na prática a formulação de modelos reológicos, básicos ou combinados, específicos para cada tipo de material. 
Diferentemente do modelo elástico, caracterizado pelo aparecimento de deformações elásticas instantâneas à aplicação de solicitações estáticas, o modelo viscoso apresenta um comportamento dependente do tempo. Com isso, mesmo que as tensões aplicadas permaneçam constantes, haverá variação das deformações ao longo do tempo. A relação fundamental do comportamento viscoso é representada por:

$$
\sigma_{i j}=\eta_{i j}^{k l} \dot{\varepsilon}_{k l}
$$

onde $\eta_{i j}^{k l}$ é a matriz viscosa escrita em função de parâmetros representativos, determinados experimentalmente, da viscosidade do material.

Para o desenvolvimento das representações integrais dos problemas viscosos, é necessária a adoção de um modelo reológico combinado que possa representar o efeito da viscosidade nos materiais. Neste trabalho, por simplicidade, adota-se o modelo viscoelástico de Kelvin-Voigt, representado pelo arranjo em paralelo de um amortecedor e de uma mola (Figura 4.1):

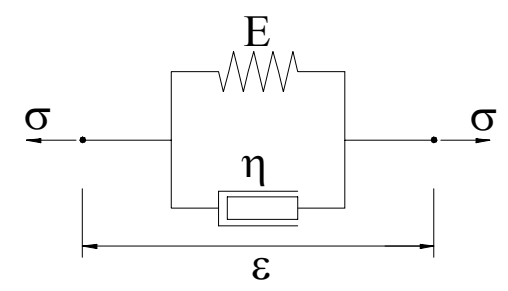

FIGURA 4.1 - Modelo viscoelástico de Kelvin-Voigt.

O modelo de Kelvin-Voigt caracteriza-se também, pela igualdade de deformações nos dois trechos:

$$
\varepsilon_{i j}=\varepsilon_{i j}^{e}=\varepsilon_{i j}^{v}
$$

onde $\varepsilon_{i j}$, $\varepsilon_{i j}^{e}$ e $\varepsilon_{i j}^{v}$ são, respectivamente, as deformações totais, elásticas e viscosas.

As tensões totais são definidas pela soma das tensões viscosas (no amortecedor) e das tensões elásticas (na mola), como:

$$
\sigma_{i j}=\sigma_{i j}^{e}+\sigma_{i j}^{v}
$$

onde as tensões elásticas e as viscosas são definidas por:

$$
\sigma_{i j}^{e}=C_{i j k l} \varepsilon_{k l}^{e}=C_{i j k l} \varepsilon_{k l}
$$




$$
\sigma_{i j}^{v}=\eta_{i j}^{k l} \dot{\varepsilon}_{k l}^{v}=\eta_{i j}^{k l} \dot{\varepsilon}_{k l}
$$

onde $C_{i j k l}$ é a matriz constitutiva elástica e $\eta_{i j}^{k l}$ é a matriz viscosa, que para o estado plano de tensão e para materiais isotrópicos, pode ser representada por:

$$
\eta=\frac{2 \theta_{\mu} \mu}{\theta_{\lambda} \lambda+2 \theta_{\mu} \mu}\left[\begin{array}{ccc}
2\left(\theta_{\lambda} \lambda+\theta_{\mu} \mu\right) & \theta_{\lambda} \lambda & 0 \\
\theta_{\lambda} \lambda & 2\left(\theta_{\lambda} \lambda+\theta_{\mu} \mu\right) & 0 \\
0 & 0 & 2\left(\theta_{\lambda} \lambda+\theta_{\mu} \mu\right)
\end{array}\right]
$$

onde $\theta_{\lambda}$ e $\theta_{\mu}$ são coeficientes representativos da viscosidade do material, determinados, respectivamente, de ensaios simples de cisalhamento e de tração. Os termos $\lambda$ e $\mu$ são as conhecidas constantes de Lamé para materiais isotrópicos, expressas pelas constantes de elasticidade da seguinte forma:

$$
\lambda=\frac{v E}{(1+v)(1-2 v)} \quad \mu=G=\frac{v E}{2(1+v)}
$$

No entanto, na grande maioria dos materiais, a matriz viscosa $\eta_{i j}^{k l}$ pode ainda ser representada de uma forma mais simples, dependendo apenas de um único parâmetro viscoso $\gamma$, que também pode variar com o tempo, associado ao envelhecimento dos materiais e derivado das funções de fluência e relaxação. Neste caso, tem-se $\gamma=\theta_{\lambda}=\theta_{\mu}$ e assim, a matriz viscosa pode ser escrita como:

$$
\eta_{i j}^{k l}=\gamma_{i j k l}
$$

A Equação (4.13) é a expressão empregada nas análises viscosas deste trabalho, sendo que $C_{i j k l}$ é agora referente a materiais anisotrópicos, salientando-se que não se dispõe na literatura de parâmetros de viscosidade específicos para materiais com anisotropia geral, abrindo-se assim, uma frente de pesquisas muito vasta principalmente para materiais poliméricos reforçados por fibras.

É importante comentar ainda, que o modelo reológico de Kelvin-Voigt, apesar de não considerar o comportamento instantâneo de deformação, é perfeitamente adequado para os propósitos iniciais de análise numérica aqui pretendidos. No entanto, a consideração de outro modelo, que possa prever deformações imediatas como, por 
exemplo, o modelo de Boltzmann (MESQUITA, 2002), é perfeitamente viável e se constitui em continuidade nas implementações a serem desenvolvidas em trabalhos futuros do SET/EESC.

Assim, no modelo de Kelvin-Voigt, utilizando-se a Equação (4.13), as tensões totais (4.8) podem ser reescritas:

$$
\sigma_{i j}=C_{i j k l} \varepsilon_{k l}+\gamma C_{i j k l} \dot{\varepsilon}_{k l} \quad\left(\text { para } \gamma=\theta_{\lambda}=\theta_{\mu}\right)
$$

Com isso, impondo-se na Equação integral (4.4) a relação reológica (4.14), obtém-se a representação integral do problema viscoelástico quase estático pelo MEF:

$$
\int_{\Gamma} \delta u_{i} p_{i} d \Gamma-\int_{\Omega} \delta \varepsilon_{i j} C_{i j k l} \varepsilon_{k l} d \Omega-\int_{\Omega} \delta \varepsilon_{i j} C_{i j k l} \dot{\varepsilon}_{k l} d \Omega+\int_{\Omega} \delta u_{i} b_{i} d \Omega=0
$$

onde se observa, que a única diferença em relação à formulação elastostática é a presença da terceira integral responsável pelo comportamento viscoso.

Ressalta-se que a Equação integral (4.15) é resultante da consideração das propriedades reológicas viscosas do material na equação de equilíbrio do corpo, Equação (4.1), e será resolvida, neste trabalho, fazendo-se uso de algoritmos de integração temporal (MESQUITA (2002), MESQUITA \& CODA (2002)), onde incrementos de tempo são considerados, tal como aqueles usualmente empregados nas análises dinâmicas, diferenciando-se assim, de outras formulações (LEMAITRE \& CHABOCHE (1990), MUNAIAR (1998)), onde a viscosidade do material é considerada fazendo-se uso de funções de fluência ou de relaxação com processos incrementais onde as solicitações são aplicadas incrementalmente.

Descreve-se a seguir a representação para o problema elastoplástico, partindo-se também da equação integral do problema elastostático, Equação (4.4). O modelo reológico do problema elastoplástico está apresentado na Figura (4.2):

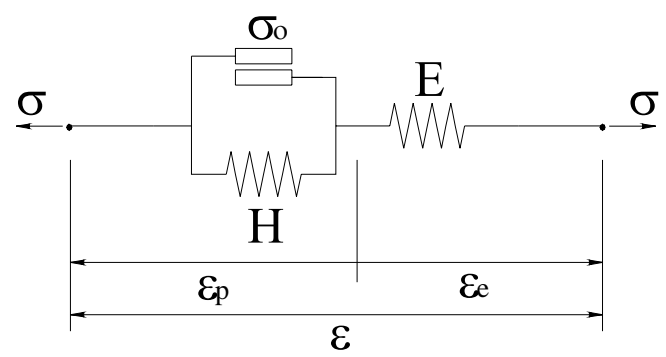

FIGURA 4.2 - Modelo reológico elastoplástico. 
onde $H$ representa o módulo plástico tangente do material e $\sigma_{0}$ a tensão de escoamento.

As deformações totais são definidas pela soma das deformações elásticas da segunda mola e das deformações plásticas referente ao conjunto bloco-mola:

$$
\varepsilon_{k l}=\varepsilon_{k l}^{e}+\varepsilon_{k l}^{p}
$$

Com as tensões sendo definidas por:

$$
\sigma_{i j}=C_{i j k l} \varepsilon_{k l}^{e}=C_{i j k l}\left(\varepsilon_{k l}-\varepsilon_{k l}^{p}\right)=C_{i j k l} \varepsilon_{k l}-\sigma_{i j}^{p}
$$

sendo $\sigma_{i j}^{p}$ chamada de tensão residual plástica numa análise elastoplástica incrementaliterativa.

Assim, impondo-se na Equação (4.4) a relação reológica elastoplástica representada pela Equação (4.17) tem-se a representação integral para o problema elastoplástico:

$$
\int_{\Gamma} \delta u_{i} p_{i} d \Gamma-\int_{\Omega} \delta \varepsilon_{i j} C_{i j k l}^{l} \varepsilon_{k l} d \Omega+\int_{\Omega} \delta \varepsilon_{i j} C_{i j k l} \varepsilon_{k l}^{p} d \Omega+\int_{\Omega} \delta u_{i} b_{i} d \Omega=0
$$

ou ainda,

$$
\int_{\Gamma} \delta u_{i} p_{i} d \Gamma-\int_{\Omega} \delta \varepsilon_{i j} C_{i j}^{k l} \varepsilon_{k l} d \Omega+\int_{\Omega} \delta \varepsilon_{i j} \sigma_{k l}^{p} d \Omega+\int_{\Omega} \delta u_{i} b_{i} d \Omega=0
$$

Assim, formulados os problemas viscoelásticos e elastoplásticos, pode-se então, apresentar a representação integral do problema viscoplástico. O modelo reológico adotado é representado pelo arranjo em série de um conjunto em paralelo bloco/mola com a respectiva mola do modelo viscoelástico de Kelvin-Voigt (Figura 4.3):

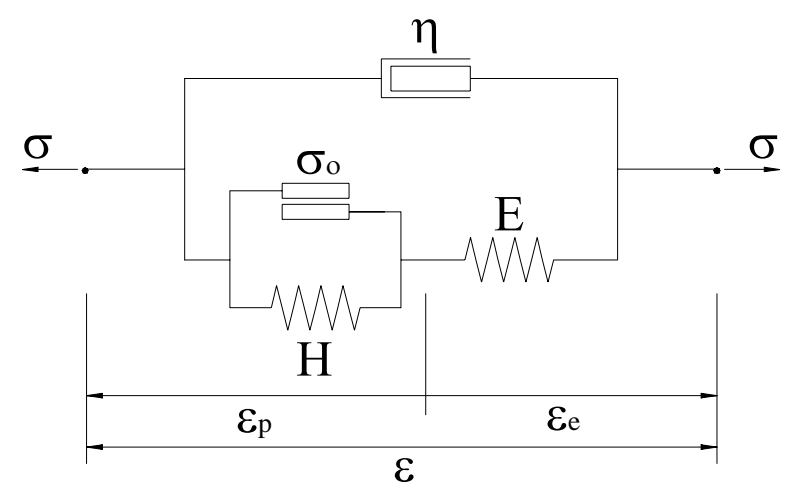

FIGURA 4.3 - Modelo reológico viscoplástico. 
Para o modelo viscoplástico adotado, as deformações são relacionadas por meio da seguinte expressão:

$$
\varepsilon_{k l}=\varepsilon_{k l}^{v}=\varepsilon_{k l}^{e}+\varepsilon_{k l}^{p} \quad \Rightarrow \quad \varepsilon_{k l}^{e}=\varepsilon_{k l}-\varepsilon_{k l}^{p}
$$

onde $\varepsilon_{k l}, \varepsilon_{k l}^{e}, \varepsilon_{k l}^{v}$ e $\varepsilon_{k l}^{p}$ são, respectivamente, as deformações totais, elásticas, viscosas e plásticas. As tensões totais são definidas pela soma das tensões viscosas (no amortecedor) e das tensões elastoplásticas (no trecho elastoplástico), como:

$$
\sigma_{i j}=\sigma_{i j}^{e p}+\sigma_{i j}^{v}
$$

Semelhantemente às deformações, $\sigma_{k l}, \sigma_{k l}^{e p}$ e $\sigma_{k l}^{v}$ são, respectivamente, as tensões totais, elastoplásticas e viscosas:

$$
\begin{aligned}
& \sigma_{i j}^{e p}=C_{i j k l} \varepsilon_{k l}^{e} \\
& \sigma_{i j}^{v}=\eta_{i j}^{k l} \dot{\varepsilon}_{k l}^{v}=\gamma C_{i j k l} \dot{\varepsilon}_{k l}
\end{aligned}
$$

onde $C_{i j k l}$ é a matriz constitutiva elástica, $\eta_{i j}^{k l}$ é a matriz viscosa definida pela Equação (4.11) e $\gamma$ é o parâmetro viscoso simplificado. Assim, as tensões totais (4.21) podem ser expressas da seguinte forma:

$$
\sigma_{i j}=C_{i j k l} \varepsilon_{k l}^{e}+\gamma C_{i j k l} \dot{\varepsilon}_{k l}=C_{i j k l}\left(\varepsilon_{k l}-\varepsilon_{k l}^{p}\right)+\gamma C_{i j k l} \dot{\varepsilon}_{k l}=C_{i j k l}\left(\varepsilon_{k l}+\gamma \dot{\varepsilon}_{k l}\right)-\sigma_{i j}^{p}
$$

Impondo-se na Equação (4.4) a relação reológica viscoplástica representada pela Equação (4.23) tem-se a representação integral para o problema viscoplástico:

$$
\begin{gathered}
\int_{\Gamma} \delta u_{i} p_{i} d \Gamma-\int_{\Omega} \delta \varepsilon_{i j} C_{i j k l} \varepsilon_{k l} d \Omega-\int_{\Omega} \delta \varepsilon_{i j} C_{i j k l} \dot{\varepsilon}_{k l} d \Omega+\int_{\Omega} \delta \varepsilon_{i j} \sigma_{k l}^{p} d \Omega+ \\
+\int_{\Omega} \delta u_{i} b_{i} d \Omega=0
\end{gathered}
$$

\subsection{TRATAMENTO ALGÉBRICO DA REPRESENTAÇÃO INTEGRAL}

Definidas as formulações dos problemas a serem abordados, pode-se então, transfor as representações integrais em equações algébricas através do MEF. Para tanto, 
deve-se discretizar o domínio do problema a ser estudado em um número adequado de elementos finitos (Figura 4.4):

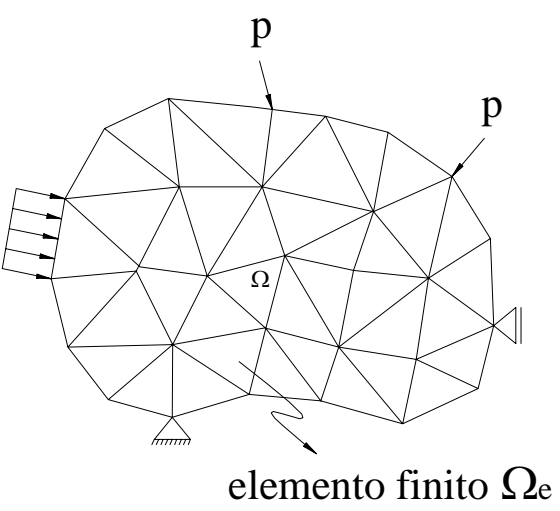

FIGURA 4.4 - Domínio discretizado em elementos finitos.

Na presente pesquisa, dois elementos finitos triangulares diferentes são utilizados, o elemento LST (Linear Strain Tringle) com aproximação linear para deformações e o elemento QST (Quadrate Strain Tringle), com aproximação quadrática para deformações, ambos apresentados na Figura (4.5):

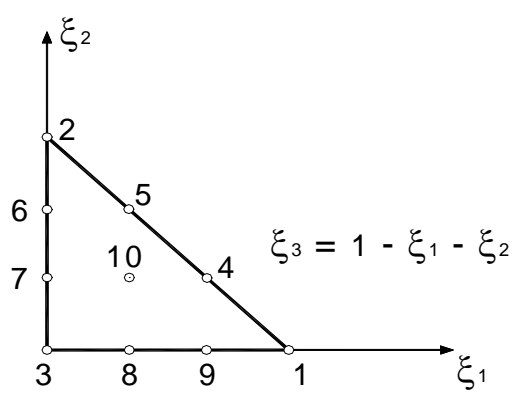

(a)

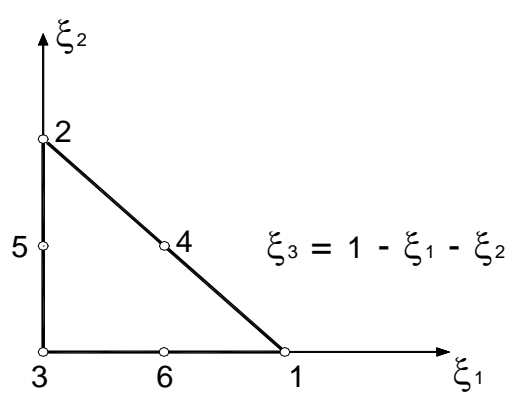

(b)

FIGURA 4.5 - (a) Elemento finito QST. (b) Elemento finito LST.

Dessa forma, as variáveis do problema são aproximadas, parametrizando-as com relação aos seus valores nodais:

$$
\begin{array}{lll}
u_{i}=\phi^{\alpha} U_{i}^{\beta} & \Rightarrow & \varepsilon_{i j}=\phi,{ }_{j}^{\alpha} U_{i}^{\beta} \\
\delta u_{i}=\phi^{\alpha} \delta U_{i}^{\beta} & \Rightarrow & \delta \varepsilon_{i j}=\phi,{ }_{j}^{\alpha} \delta U_{i}^{\beta} \\
\dot{u}_{i}=\phi^{\alpha} \dot{U}_{i}^{\beta} & \Rightarrow & \dot{\varepsilon}_{i j}=\phi,{ }_{j}^{\alpha} \dot{U}_{i}^{\beta} \\
b_{i}=\phi^{\alpha} b_{i}^{\alpha} ; & p_{i}=\tilde{\phi}^{\alpha} P_{i}^{\alpha}
\end{array}
$$


onde $\tilde{\phi}$ é a função de forma adotada para interpolar as forças de superfície e $\phi$ é a função de forma adotada para interpolar o restante das variáveis e o subscrito $\alpha$ referese aos nós do elemento finito. Deve-se notar que para a aproximação no contorno $\alpha$ corresponde ao número de nós pertencente à superfície onde $p_{i}$ é aproximado. Os termos $U_{i}^{\beta}, \dot{U}_{i}^{\beta}, \delta U_{i}^{\beta}, P_{i}^{\alpha}$ e $b_{i}^{\alpha}$ são valores nodais de deslocamento, velocidade, deslocamento virtual, forças de superfície e forças volumétricas respectivamente. Substituindo-se as expressões em (4.25) na representação integral em (4.24) encontrase:

$$
\begin{aligned}
& \delta U_{i}^{\alpha}\left[\sum_{s=1}^{n_{s}} \int_{\Gamma_{s}} \phi^{\alpha} \widetilde{\phi}^{\beta} d \Gamma_{s} P_{i}^{\beta}-\sum_{e=1}^{n_{e}} \int_{\Omega_{e}} \phi{ }_{j}^{\alpha} C_{i j}^{l m} \phi{ }_{m_{m}}^{\beta} d \Omega_{e} U_{l}^{\beta}-\right. \\
& \left.\sum_{e=1}^{n_{e}} \int_{\Omega_{e}} \phi{ }_{j}^{\alpha} \gamma C_{i j}^{l m} \phi{ }_{m_{m}}^{\beta} d \Omega_{e} \dot{U}_{l}^{\beta}+\sum_{e=1}^{n_{e}} \int_{\Omega_{e}} \phi{ }_{j}{ }^{\alpha} \cdot \sigma_{j i}^{p}\left(\xi_{i}\right) d \Omega_{e}+\sum_{e=1}^{n_{e}} \int_{\Omega_{e}} \phi^{\alpha} \phi^{\beta} d \Omega_{e} b_{i}^{\beta}\right]=0
\end{aligned}
$$

onde $n_{s}$ é o número de superfícies solicitadas, $n_{e}$ é o número de elementos finitos e $\xi_{i}$ são coordenadas admensionais do elemento finito triangular, referentes aos pontos de integração de Hammer, utilizados na integração numérica e onde são calculadas as deformações e as tensões.

Deve-se notar que os deslocamentos virtuais $\delta U_{i}^{\alpha}$ presentes na expressão (4.26) podem ser quaisquer, logo, para que a igualdade em (4.26) seja verificada tem-se:

$$
\begin{aligned}
& \sum_{e=1}^{n_{e}} \int_{\Omega_{e}} \phi{ }_{, j}^{\alpha} C_{i j}^{l m} \phi{ }_{{ }_{m}}^{\beta} d \Omega_{e} U_{l}^{\beta}+\sum_{e=1}^{n_{e}} \int_{\Omega_{e}} \phi{ }_{, j}^{\alpha} \gamma C_{i j}^{l m} \phi{ }_{{ }_{m}}^{\beta} d \Omega_{e} \dot{U}_{l}^{\beta}=\sum_{s=1}^{n_{s}} \int_{\Gamma_{s}} \phi^{\alpha} \tilde{\phi}^{\beta} d \Gamma_{s} P_{i}^{\beta}+ \\
& \sum_{e=1}^{n_{e}} \int_{\Omega_{e}} \phi{ }_{, j}{ }^{\alpha} \cdot \sigma_{j i}^{p}\left(\xi_{i}\right) d \Omega_{e}+\sum_{e=1}^{n_{e}} \int_{\Omega_{e}} \phi^{\alpha} \phi^{\beta} d \Omega_{e} b_{i}^{\beta}
\end{aligned}
$$

Com isso, a Equação (4.27) pode ser escrita de forma matricial como:

$$
K U+\gamma K \dot{U}=G P+F_{p}+B b+F
$$

onde, $G$ e $B$ são matrizes que transformam forças distribuídas no contorno, ou no domínio do corpo, em forças nodais equivalentes; $F$ é o vetor de forças concentradas aplicadas diretamente nos nós; $F_{p}$ é o vetor de forças residuais obtidas das tensões plásticas $\sigma_{p}$ integradas no domínio dos elementos finitos; $\dot{U}$ é o vetor de velocidades 
nodais. O sistema de equações diferenciais no tempo é resolvido adotando-se aproximação linear no tempo para $\dot{U}$, isto é:

$$
\left(\frac{U_{t+\Delta t}-U_{t}}{\Delta_{t}}\right)=\dot{U}_{t+\Delta t}
$$

Ao se substituir a aproximação (4.29) na Equação (4.28) obtém-se um processo de análise em marcha, semelhante ao descrito em detalhes no capítulo 5 para o MEC. Salienta-se ainda, que com a utilização dos dois elementos finitos da Figura 4.5, uma importante constatação sobre aproximações foi realizada nesta pesquisa. Para meios fortemente anisotrópicos, ou seja, meios com anisotropia geral (não isotrópicos e não ortotrópicos), é preciso uma aproximação no mínimo quadrática para as deformações (QST) no intuito de se obter bons resultados para as tensões (VANALLI et al., 2003a). Nos exemplos que são apresentados no decorrer do presente texto, os resultados obtidos com o uso das duas aproximações podem ser verificados.

Assim, finaliza-se este capítulo sobre formulações do MEF para problemas anisotrópicos e a seguir, as formulações para o MEC são descritas. 


\section{FORMULAÇÃO DO MEC PARA O PROBLEMA VISCOPLÁSTICO ANISOTRÓPICO}

Neste capítulo, apresenta-se a formulação do Método dos Elementos de Contorno (MEC) para a análise de problemas viscoplásticos em meios anisotrópicos, abordando-se os problemas elásticos, viscoelásticos e elastoplásticos.

\subsection{FORMULAÇÃO INTEGRAL PARA A ABORDAGEM DO PROBLEMA}

O princípio básico do MEC é a transformação das equações diferenciais, que regem um determinado problema físico, em equações integrais de contorno com variáveis de contorno nos integrandos. De posse das equações integrais de contorno, o MEC consiste em se adotar uma aproximação numérica para estas, obtendo-se um conjunto de equações algébricas que permitem a resolução de diversos problemas cujas soluções não são conhecidas explicitamente.

Para a formulação das equações integrais de contorno que regem um determinado problema, independente do tipo do material que constitui o meio analisado, pode se utilizar a técnica dos resíduos ponderados empregada sobre a equação de equilíbrio do problema em consideração, ou seja:

$$
\int_{\Omega} u_{k i}^{*}\left(\sigma_{i j, j}+b_{i}\right) d \Omega=0=\int_{\Omega} R_{i} u_{k i}^{*} d \Omega
$$


onde o ponderador $u_{k i}^{*}$ é denominada solução fundamental e representa o efeito (deslocamento na direção i) de uma carga concentrada unitária estática atuando (na direção $k$ ) em um ponto de um domínio infinito elástico e homogêneo. Integrando-se por partes o primeiro termo da Equação (5.1), tem-se:

$$
\int_{\Gamma} u_{k i}^{*} \sigma_{i j} \eta_{j} d \Gamma-\int_{\Omega} u_{k i, j}^{*} \sigma_{i j} d \Omega+\int_{\Omega} u_{k i}^{*} b_{i} d \Omega=0
$$

sendo $\Gamma$ a variável que define o contorno do corpo e $\eta_{j}$ a componente do versor normal à superfície. Sabendo-se que $\sigma_{i j} \eta_{j}=p_{i}$ e que $u_{k i, j}^{*} \sigma_{i j}=\varepsilon_{k i, j}^{*} \sigma_{i j}$, onde $\varepsilon_{k i j}^{*}$ é a deformação fundamental, a Equação (5.2) torna-se:

$$
\int_{\Gamma} u_{k i}^{*} p_{i} d \Gamma-\int_{\Omega} \varepsilon_{k i j}^{*} \sigma_{i j} d \Omega+\int_{\Omega} u_{k i}^{*} b_{i} d \Omega=0
$$

Semelhantemente à Equação (4.4) para o MEF, a Equação (5.3) é o ponto de partida para a obtenção das representações integrais do MEC. Na Equação (5.3) podese impor as relações reológicas dos problemas que se queira formular no sentido de se obter as representações integrais para estes. Para o caso elástico a expressão (5.3) torna-se:

$$
\int_{\Gamma} u_{k i}^{*} p_{i} d \Gamma-\int_{\Omega} \varepsilon_{k i j}^{*} C_{i j}^{l m} \varepsilon_{l m} d \Omega+\int_{\Omega} u_{k i}^{*} b_{i} d \Omega=0
$$

Sabendo-se que:

$$
\varepsilon_{k i j}^{*} C_{i j}^{l m} \varepsilon_{l m}=\sigma_{k l m}^{*} \varepsilon_{l m}=\sigma_{k l m}^{*} u_{l, m}=\sigma_{k i j}^{*} u_{i, j}
$$

a Equação (5.4) torna-se:

$$
\int_{\Gamma} u_{k i}^{*} p_{i} d \Gamma-\int_{\Omega} \sigma_{k i j}^{*} u_{i, j} d \Omega+\int_{\Omega} u_{k i}^{*} b_{i} d \Omega=0
$$

Aplicando-se integração por partes na segunda integral da Equação (5.6) resulta:

$$
\int_{\Gamma} u_{k i}^{*} p_{i} d \Gamma-\int_{\Gamma} \sigma_{k i j}^{*} \eta_{j} u_{i} d \Gamma+\int_{\Omega} \sigma_{k i j, j}^{*} u_{i} d \Omega+\int_{\Omega} u_{k i}^{*} b_{i} d \Omega=0
$$

A Equação integral (5.7) pode ser reescrita fazendo-se uso da equação de equilíbrio fundamental definida por: 


$$
\sigma_{k i j, j}^{*}=-\delta(s, p) \delta_{k i}
$$

sendo $\delta(s, p)$ o delta de Dirac, $p$ refere-se a uma posição do domínio do corpo e $s$ representa a posição do ponto fonte. Aplicando-se a Equação (5.8) em (5.7) e levandose em consideração as propriedades do delta de Dirac e que $\sigma_{k i j}^{*} \eta_{j}=p_{k i}^{*}$, encontra-se:

$$
\bar{C}_{k i} u_{i}(p)=\int_{\Gamma} u_{k i}^{*} p_{i} d \Gamma-\int_{\Gamma} p_{k i}^{*} u_{i} d \Gamma+\int_{\Omega} u_{k i}^{*} b_{i} d \Omega=0
$$

onde o termo $\bar{C}_{k i}$ é dependente da posição do ponto fonte $s$ em relação ao corpo analisado. Para pontos fontes no contorno do corpo, este termo é definido como uma matriz (Equação 5.10), onde os elementos são funções da geometria do contorno (Figura 5.1):

$$
\bar{C}_{k i}(s)=\left[\begin{array}{cc}
A & B \\
C & D
\end{array}\right]
$$

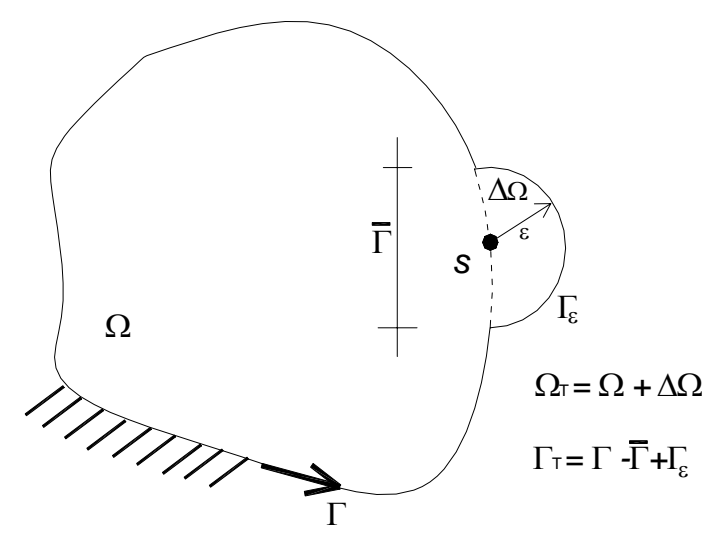

FIGURA 5.1 - Ponto fonte no contorno, interno a uma região idealizada.

Sendo que, para problemas 2D, os valores de $A, B, C$ e $D$ também dependem da relação constitutiva, a saber, isotrópica ou anisotrópica. Por simplicidade, nos problemas anisotrópicos $\bar{C}_{k i}(s)$ é determinado via movimento de corpo-rígido (TELLES \& BREBBIA, 1982; LIU, 2000). O termo $p_{k i}^{*}$ é a solução fundamental em forças de superfície.

A Equação (5.9) é a representação integral da formulação elastostática do MEC aqui adotada. As integrais presentes na Equação (5.9) possuem singularidades que podem ser tratadas através da utilização de ponto fonte exterior ao domínio do problema analisado, ou solucionadas analiticamente ou semi-analiticamente. 
Para a consideração dos problemas com viscosidade é preciso assumir as mesmas hipóteses adotadas na formulação desses problemas com o MEF, item 4.1, fazendo-se uso de algoritmos de integração temporal (MESQUITA (2002), MESQUITA \& CODA (2002)), onde incrementos de tempo são considerados, tal como aqueles usualmente empregados nas análises dinâmicas. Essa técnica aqui utilizada permite ainda que a viscosidade seja representada apenas com integrais de contorno, tornando-se possível modelar esses problemas no contorno do corpo e preservando as vantagens inerentes ao MEC.

Uma outra maneira de tratar os problemas viscosos seria com a formulação onde 0 termo viscoso é definido por integrais de domínio (TELLES \& BREBBIA, 1982). Neste caso, a vantagem de redução das dimensões para problemas lineares fica perdida, requerendo integrações de domínios com núcleos singulares semelhantes aos de plasticidade (MI \& ALIABADI, 1992; LEITÃO et al., 1995), requerendo, dessa maneira, um maior custo computacional em relação à técnica aqui empregada. Deve-se comentar que para problemas viscoplásticos ou elastoplásticos integrais de domínio limitadas à região de plastificação são necessárias.

Para a obtenção das equações integrais do problema viscoso, o procedimento inicial é o mesmo descrito anteriormente para a formulação do MEF, ou seja, aplica-se a relação constitutiva do modelo de Kelvin-Voigt, Equação (4.14), sobre a representação integral (5.3). Assim:

$$
\int_{\Gamma} u_{k i}^{*} p_{i} d \Gamma-\int_{\Omega} \varepsilon_{k i j}^{*} C_{i j}^{l m} \varepsilon_{l m} d \Omega-\int_{\Omega} \varepsilon_{k i j}^{*} C_{i j}^{l m} \dot{\varepsilon}_{l m} d \Omega+\int_{\Omega} u_{k i}^{*} b_{i} d \Omega=0
$$

Sabendo-se ainda que:

$$
\begin{aligned}
& \varepsilon_{k i j}^{*} C_{i j}^{l m} \varepsilon_{l m}=\sigma_{k l m}^{*} u_{l, m}=\sigma_{k i j}^{*} u_{i, j} \\
& \varepsilon_{k i j}^{*} C_{i j}^{l m} \dot{\varepsilon}_{l m}=\gamma \sigma_{k l m}^{*} \dot{u}_{l, m}=\gamma \sigma_{k i j}^{*} \dot{u}_{i, j}
\end{aligned}
$$

a Equação (5.11) torna-se:

$$
\int_{\Gamma} u_{k i}^{*} p_{i} d \Gamma-\int_{\Omega} \sigma_{k i j}^{*} u_{i, j} d \Omega-\gamma \int_{\Omega} \sigma_{k i j}^{*} \dot{u}_{i, j} d \Omega+\int_{\Omega} u_{k i}^{*} b_{i} d \Omega=0
$$

Aplicando-se integração por partes na segunda e na terceira integral da Equação (5.14) encontra-se: 


$$
\begin{gathered}
\int_{\Gamma} u_{k i}^{*} p_{i} d \Gamma-\int_{\Gamma} \sigma_{k i j}^{*} \eta_{j} u_{i} d \Gamma+\int_{\Omega} \sigma_{k i j, j}^{*} u_{i} d \Omega-\gamma \int_{\Gamma} \sigma_{k i j}^{*} \eta_{j} \dot{u}_{i} d \Gamma+ \\
+\gamma \int_{\Omega} \sigma_{k i j, j}^{*} \dot{u}_{i} d \Omega+\int_{\Omega} u_{k i}^{*} b_{i} d \Omega=0
\end{gathered}
$$

A Equação integral (5.15) pode ser reescrita fazendo-se uso da equação de equilíbrio fundamental (5.8), das propriedades do delta de Dirac e de que $\sigma_{k i j}^{*} \eta_{j}=p_{k i}^{*}$. Assim:

$$
\bar{C}_{k i} u_{i}(p)+\gamma \bar{C}_{k i} \dot{u}_{i}(p)=\int_{\Gamma} u_{k i}^{*} p_{i} d \Gamma-\int_{\Gamma} p_{k i}^{*} u_{i} d \Gamma-\gamma \int_{\Gamma} p_{k i}^{*} \dot{u}_{i} d \Gamma+\int_{\Omega} u_{k i}^{*} b_{i} d \Omega
$$

A Equação (5.16) é a representação integral da formulação viscoelástica do MEC para o modelo reológico de Kelvin-Voigt. Observa-se que a diferença entre esta representação e a representação elastostática, Equação (5.9), é a presença do segundo termo no lado esquerdo e da terceira integral do lado direito da Equação (5.16), ambos responsáveis pelo comportamento viscoso.

Com o problema viscoelástico formulado, pode-se então partir para a representação integral do problema elastoplástico. Para tanto, é preciso considerar a relação reológica do modelo elastoplástico, Equação (4.17), na Equação integral (5.3):

$$
\int_{\Gamma} u_{k i}^{*} p_{i} d \Gamma-\int_{\Omega} \varepsilon_{k i j}^{*} C_{i j}^{l m} \varepsilon_{l m} d \Omega+\int_{\Omega} \varepsilon_{k i j}^{*} \sigma_{i j}^{p} d \Omega+\int_{\Omega} u_{k i}^{*} b_{i} d \Omega=0
$$

A terceira integral presente na Equação (5.17) é responsável pela contribuição da plastificação no corpo. Sabendo-se ainda que:

$$
\varepsilon_{k i j}^{*} C_{i j}^{l m} \varepsilon_{l m}=\sigma_{k l m}^{*} u_{l, m}=\sigma_{k i j}^{*} u_{i, j}
$$

a Equação (5.17) torna-se:

$$
\int_{\Gamma} u_{k i}^{*} p_{i} d \Gamma-\int_{\Omega} \sigma_{k i j}^{*} u_{i, j} d \Omega+\int_{\Omega} \varepsilon_{k i j}^{*} \sigma_{i j}^{p} d \Omega+\int_{\Omega} u_{k i}^{*} b_{i} d \Omega=0
$$

Aplicando-se integração por partes na segunda integral da Equação (5.19) encontra-se:

$$
\int_{\Gamma} u_{k i}^{*} p_{i} d \Gamma-\int_{\Gamma} \sigma_{k i j}^{*} \eta_{j} u_{i} d \Gamma+\int_{\Omega} \sigma_{k i j, j}^{*} u_{i} d \Omega+\int_{\Gamma} \varepsilon_{k i j}^{*} \sigma_{i j}^{p} d \Gamma+\int_{\Omega} u_{k i}^{*} b_{i} d \Omega=0
$$


A Equação integral (5.20) pode também ser reescrita fazendo-se uso da equação de equilíbrio fundamental (5.8), das propriedades do delta de Dirac e ainda considerando-se que $\sigma_{k i j}^{*} \eta_{j}=p_{k i}^{*}$. Assim:

$$
\bar{C}_{k i} u_{i}(p)=\int_{\Gamma} u_{k i}^{*} p_{i} d \Gamma-\int_{\Gamma} p_{k i}^{*} u_{i} d \Gamma+\int_{\Omega} \varepsilon_{k i j}^{*} \sigma_{i j}^{p} d \Omega+\int_{\Omega} u_{k i}^{*} b_{i} d \Omega=0
$$

A Equação (5.21) é a representação integral da formulação elastoplástica pelo MEC. Observa-se que a diferença entre esta representação e a representação elastostática, Equação (5.9), é a presença da terceira integral do lado direito da Equação (5.21), responsável pelo comportamento plástico. Esta integral de domínio, para o caso bidimensional, apresenta singularidade do tipo $1 / r$ e será tratada utilizando-se células de domínio. Com as formulações viscoelástica e elastoplástica apresentadas, descrevese, a seguir, a representação integral para o problema viscoplástico.

Considera-se o modelo viscoplástico representado pela Equação (4.23). A imposição desta equação sobre a representação integral (5.3) resulta em:

$$
\int_{\Gamma} u_{k i}^{*} p_{i} d \Gamma-\int_{\Omega} \varepsilon_{k i j}^{*} C_{i j}^{l m} \varepsilon_{l m} d \Omega-\int_{\Omega} \varepsilon_{k i j}^{*} \gamma C_{i j}^{l m} \dot{\varepsilon}_{l m} d \Omega+\int_{\Omega} u_{k i}^{*} b_{i} d \Omega+\int_{\Omega} \varepsilon_{k i j}^{*} \sigma_{i j}^{p} d \Omega=0
$$

Utilizando-se as Equações (5.12-5.13) é possível escrever a Equação (5.22) como:

$$
\int_{\Gamma} u_{k i}^{*} p_{i} d \Gamma-\int_{\Omega} \sigma_{k i j}^{*} u_{i, j} d \Omega-\gamma \int_{\Omega} \sigma_{k i j}^{*} \dot{u}_{i, j} d \Omega+\int_{\Omega} u_{k i}^{*} b_{i} d \Omega+\int_{\Omega} \varepsilon_{k i j}^{*} \sigma_{i j}^{p} d \Omega=0
$$

Aplicando-se integração por partes na segunda e na terceira integral da Equação (5.23) encontra-se:

$$
\begin{gathered}
\int_{\Gamma} u_{k i}^{*} p_{i} d \Gamma-\int_{\Gamma} \sigma_{k i j}^{*} \eta_{j} u_{i} d \Gamma+\int_{\Omega} \sigma_{k i j, j}^{*} u_{i} d \Omega-\gamma \int_{\Gamma} \sigma_{k i j}^{*} \eta_{j} \dot{u}_{i} d \Gamma+ \\
+\gamma \int_{\Omega} \sigma_{k i j, j}^{*} \dot{u}_{i} d \Omega+\int_{\Omega} u_{k i}^{*} b_{i} d \Omega+\int_{\Omega} \varepsilon_{k i j}^{*} \sigma_{i j}^{p} d \Omega=0
\end{gathered}
$$

A Equação integral (5.24) pode ser reescrita fazendo-se uso da equação de equilíbrio fundamental (5.8), das propriedades do delta de Dirac e de $\sigma_{k i j}^{*} \eta_{j}=p_{k i}^{*}$. Assim: 


$$
\begin{aligned}
\bar{C}_{k i} u_{i}(p)+\gamma \bar{C}_{k i} \dot{u}_{i}(p) & =\int_{\Gamma} u_{k i}^{*} p_{i} d \Gamma-\int_{\Gamma} p_{k i}^{*} u_{i} d \Gamma-\gamma \int_{\Gamma} p_{k i}^{*} \dot{u}_{i} d \Gamma+ \\
& +\int_{\Omega} u_{k i}^{*} b_{i} d \Omega+\int_{\Omega} \varepsilon_{k i j}^{*} \sigma_{i j}^{p} d \Omega
\end{aligned}
$$

A Equação (5.25) é a representação integral de contorno da formulação viscoplástica do MEC que leva em consideração o modelo reológico apresentado na Figura (4.3). Observa-se que a diferença entre esta representação e a representação viscoelástica, Equação (5.16), é a presença da última integral do lado direito da Equação (5.25), responsável pelo comportamento plástico do corpo.

Com as representações integrais dos problemas apresentadas, pode-se então descrever a representação integral para tensões em pontos internos para o problema viscoplástico e, dessa forma, como conseqüência, abordar também as representações de tensões para os problemas viscoelástico e elastoplástico.

\subsubsection{Representação Integral para Tensões em Pontos Internos}

Com o problema de contorno formulado e resolvido, pode-se então partir para a determinação das deformações e das tensões em pontos internos. Sabendo-se que para pontos internos o termo $\bar{C}_{k i}$ é igual a $\delta_{k i}\left(\bar{C}_{k i}=\delta_{k i}\right)$, a Equação integral para o problema viscoplástico torna-se:

$$
\begin{aligned}
u_{k}(p)+\gamma \dot{u}_{k}(p)=\int_{\Gamma} u_{k i}^{*} p_{i} d \Gamma-\int_{\Gamma} p_{k i}^{*} u_{i} d \Gamma-\gamma \int_{\Gamma} p_{k i}^{*} \dot{u}_{i} d \Gamma+ \\
\quad+\int_{\Omega} u_{k i}^{*} b_{i} d \Omega+\int_{\Omega} \varepsilon_{k i j}^{*} \sigma_{i j}^{p} d \Omega
\end{aligned}
$$

Diferenciando-se a Equação (5.26) em relação à posição do ponto fonte e aplicando-se a definição de pequenas deformações, Equação (5.27):

$$
\varepsilon_{k l}=\frac{1}{2}\left(u_{k, l}+u_{l, k}\right)
$$

encontra-se a representação integral para as deformações (BUI, 1978; TELLES \& BREBBIA, 1982; VENTURINI, 1982): 


$$
\begin{aligned}
\varepsilon_{k l}(p)+\gamma \dot{\varepsilon}_{k l}(p)=\int_{\Gamma} \varepsilon_{k l i}^{*} p_{i} d \Gamma-\int_{\Gamma} p_{k l i}^{*} u_{i} d \Gamma-\gamma \int_{\Gamma} p_{k l i}^{*} \dot{u}_{i} d \Gamma+ \\
\quad+\int_{\Omega} \varepsilon_{k l i}^{*} b_{i} d \Omega+\int_{\Omega} t_{k l i j}^{*} \sigma_{i j}^{p} d \Omega+\widehat{g}_{k l i j} \sigma_{i j}^{p}(p)
\end{aligned}
$$

lembrando-se que as deformações $\varepsilon_{k l}(p)$, segundo o modelo reológico viscoplástico adotado (Figura 4.3), são iguais a:

$$
\varepsilon_{k l}=\varepsilon_{k l}^{v}=\varepsilon_{k l}^{e}+\varepsilon_{k l}^{p}
$$

Observa-se que, como a diferenciação é feita em relação ao ponto fonte, apenas os valores fundamentais necessitam ser diferenciados. Aplicando-se agora a lei constitutiva anisotrópica ( $C_{i j}^{l m}$ ) sobre a equação de deformações (5.28), obtém-se, de forma direta, a equação integral para tensões:

$$
\begin{aligned}
\bar{\sigma}_{\rho q}(p)+\sigma_{\rho q}^{v}(p)= & \int_{\Gamma} \sigma_{\rho q i}^{*} p_{i} d \Gamma-\int_{\Gamma} p_{\rho q i}^{*} u_{i} d \Gamma-\gamma \int_{\Gamma} p_{\rho q i}^{*} \dot{u}_{i} d \Gamma+ \\
& +\int_{\Omega} \sigma_{\rho q i}^{*} b_{i} d \Omega+\int_{\Omega} t_{\rho q i j}^{*} \sigma_{i j}^{p} d \Omega+\widehat{g}_{\rho q i j} \sigma_{i j}^{p}(p)
\end{aligned}
$$

onde as tensões $\bar{\sigma}_{\rho q}$, segundo a relação (5.29), equivalem a:

$$
\bar{\sigma}_{\rho q}=\sigma_{\rho q}^{e p}+\sigma_{\rho q}^{p}
$$

Assim, recorrendo-se também à expressão (4.21), a Equação (5.30) pode ser reescrita como:

$$
\begin{aligned}
\sigma_{\rho q}(p) & =\int_{\Gamma} \sigma_{\rho q i}^{*} p_{i} d \Gamma-\int_{\Gamma} p_{\rho q i}^{*} u_{i} d \Gamma-\gamma \int_{\Gamma} p_{\rho q i}^{*} \dot{u}_{i} d \Gamma+ \\
& +\int_{\Omega} \sigma_{\rho q i}^{*} b_{i} d \Omega+\int_{\Omega} t_{\rho q i j}^{*} \sigma_{i j}^{p} d \Omega+\widehat{g}_{\rho q i j} \sigma_{i j}^{p}(p)-\sigma_{\rho q}^{p}(p)
\end{aligned}
$$

onde $\sigma_{\rho q}$ é a tensão total (real) indicada na extremidade da representação do modelo reológico (Figura 4.3). Sabendo-se que $\sigma_{\rho q}^{p}=\delta_{\rho i} \delta_{q j} \sigma_{i j}^{p}$ tem-se ainda:

$$
\begin{aligned}
\sigma_{\rho q}(p)=\int_{\Gamma} \sigma_{\rho q i}^{*} p_{i} d \Gamma-\int_{\Gamma} p_{\rho q i}^{*} u_{i} d \Gamma-\gamma \int_{\Gamma} p_{\rho q i}^{*} \dot{u}_{i} d \Gamma+ \\
\quad+\int_{\Omega} \sigma_{\rho q i}^{*} b_{i} d \Omega+\int_{\Omega} t_{\rho q i j}^{*} \sigma_{i j}^{p} d \Omega+\bar{g}_{\rho q i j} \sigma_{i j}^{p}(p)
\end{aligned}
$$


que é a representação integral para tensões totais em pontos internos para o problema viscoplástico anisotrópico. Observa-se também, que na ausência de tensões plásticas residuais $\left(\sigma_{i j}^{p}\right)$, a Equação (5.32) se torna a representação para as tensões internas do problema viscoelástico, ou ainda, considerando-se juntamente a ausência do parâmetro de viscosidade $(\gamma)$, recai-se na representação do problema elastostático.

Em relação ao que foi exposto, são pertinentes ainda alguns comentários. Observa-se que a diferenciação da integral de domínio com núcleo $\varepsilon_{k i j}^{*}$ na Equação (5.26) deu origem a um termo adicional $\left(\widehat{g}_{k l i j}\right)$ na Equação (5.28) conhecido como termo livre, que é oriundo de derivações sobre integrais singulares (BUI, 1978). Esse termo é bastante conhecido, e utilizado, em aplicações sobre plasticidade para meios isotrópicos onde se emprega a solução fundamental de Kelvin (CODA, 2000).

No entanto, para meios anisotrópicos gerais, onde a solução fundamental de CRUSE \& SWEDLOW (1971) é utilizada, a expressão do termo livre não é conhecida analiticamente. Nesta pesquisa a determinação deste termo é feita de forma numérica, maneira essa que já constitui contribuição original ao MEC. O detalhamento deste procedimento está apresentado no Anexo 2.

Comenta-se também, sobre a Equação (5.33), que sua quinta integral possui singularidade $1 / r^{2}$ para o caso bidimensional e esta deve ser calculada no sentido do valor principal de Cauchy ( ALIABADI et al. (1985), CISILINO (1997)), sendo tratada utilizando-se células de domínio e técnicas de subtração de singularidades (CODA, 2000).

Observa-se ainda, na Equação (5.33), que não é possível se escrever as equações integrais em pontos internos para as tensões elásticas e viscosas, ao contrário das tensões totais que são obtidas por meio da representação integral (5.33).

Para a obtenção destas tensões, adota-se um procedimento simples e eficiente proposto por MESQUITA \& CODA (2002), onde as tensões elásticas são obtidas pela solução aproximada de uma equação diferencial. Assim, para o problema viscoplástico, a equação diferencial necessária pode ser encontrada reescrevendo-se a Equação (4.23) em forma de taxas da seguinte maneira:

$$
\dot{\sigma}_{i j}^{e p}=C_{i j}^{k l} \dot{\varepsilon}_{k l}^{e}=\frac{1}{\gamma} \gamma C_{i j}^{l m}\left(\dot{\varepsilon}_{l m}-\dot{\varepsilon}_{l m}^{P}\right)=\frac{1}{\gamma} \sigma_{i j}^{v}-\dot{\sigma}_{i j}^{p} \Rightarrow \sigma_{i j}^{v}=\gamma \dot{\sigma}_{i j}^{e p}+\gamma \dot{\sigma}_{i j}^{p}
$$


Substituindo-se agora a Equação (5.34) na Equação (5.35):

$$
\sigma_{i j}=\sigma_{i j}^{e p}+\sigma_{i j}^{v}
$$

é possível encontrar o seguinte conjunto de equações diferenciais:

$$
\gamma \dot{\sigma}_{i j}^{e p}+\sigma_{i j}^{e p}+\gamma \dot{\sigma}_{i j}^{p}-\sigma_{i j}=0
$$

É importante notar que as taxas presentes na Equação (5.36) são derivadas temporais, e assim, as tensões elásticas são obtidas numericamente pela resolução da Equação (5.36) usando-se aproximação linear para as tensões, ou seja:

$$
\begin{aligned}
& \dot{\sigma}_{i j}^{e p}=\frac{\sigma_{i j(t+\Delta t)}^{e p}-\sigma_{i j(t)}^{e p}}{\Delta t} \\
& \dot{\sigma}_{i j}^{p}=\frac{\sigma_{i j(t+\Delta t)}^{p}-\sigma_{i j(t)}^{p}}{\Delta t}
\end{aligned}
$$

Substituindo-se as Equações (5.37-5.38) na Equação (5.36) resulta:

$$
\sigma_{i j(t+\Delta t)}^{e p}=\left(1+\frac{\gamma}{\Delta t}\right)^{-1} \cdot\left\{\left[\sigma_{i j}-\frac{\gamma}{\Delta t} \sigma_{i j}^{p}\right]_{(t+\Delta t)}+\frac{\gamma}{\Delta t}\left(\sigma_{i j}^{p}+\sigma_{i j}^{e p}\right)_{(t)}\right\}
$$

As tensões viscosas podem ser obtidas simplesmente pela expressão:

$$
\sigma_{i j}^{v}=\sigma_{i j}-\sigma_{i j}^{e p}
$$

Assim, com as representações integrais para tensões no domínio apresentadas, descreve-se a seguir, o método empregado para a determinação das tensões no contorno.

\subsubsection{Determinação das Tensões Totais no Contorno}

As tensões no contorno são obtidas em função das forças de superfície e dos deslocamentos, ambos determinados na solução do problema de contorno, e também, fazendo-se uso das relações constitutivas. Uma outra forma de se obter as tensões seria por meio da aplicação da Equação (5.33) no contorno, porém, essa forma não é utilizada neste trabalho.

Para exemplificar a técnica empregada, considera-se a Figura 5.2, onde um sistema cartesiano local de coordenadas está localizado em um ponto do contorno onde 
se deseja calcular as componentes de tensão, para o caso bidimensional, ou seja, $\sigma_{11}$, $\sigma_{22}$ e $\sigma_{12}$.

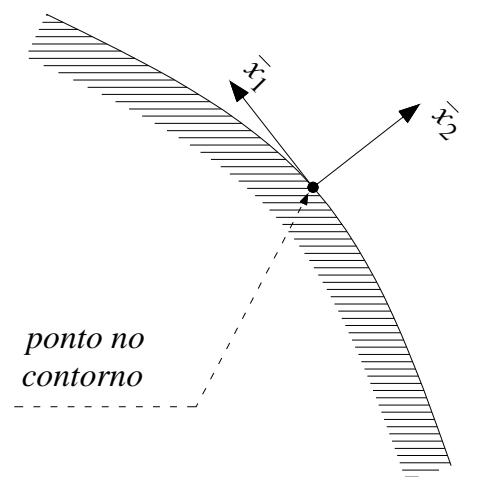

FIGURA 5.2 - Sistema cartesiano local de coordenadas.

De acordo com a Figura 5.2, pode-se escrever que:

$$
\begin{aligned}
& \bar{\sigma}_{22}=\bar{p}_{2} \\
& \bar{\sigma}_{12}=\bar{p}_{1}
\end{aligned}
$$

onde os sobrescritos indicam que os valores das componentes são referentes ao sistema local de coordenadas. Para se determinar a componente de tensão $\bar{\sigma}_{11}$, é preciso considerar a relação constitutiva e a componente de deformação $\bar{\varepsilon}_{11}$ do elemento de contorno onde se localiza o ponto em questão.

É importante observar que, quando se resolve o problema de contorno, obtém-se as forças de superfície e os deslocamentos nodais no sistema global de coordenadas, porém, para se encontrar as tensões no contorno é necessário transformar essas grandezas, e também, o tensor constitutivo, no caso anisotrópico, para o sistema local do elemento de contorno por meio da matriz de transformação entre os dois sistemas.

Definido-se o campo de deslocamentos sobre um elemento de contorno, o campo de deformações é obtido pela diferenciação do campo de deslocamentos. Desta forma, para a componente $\bar{\varepsilon}_{11}$ tem-se:

$$
\bar{\varepsilon}_{11}=\frac{\partial \bar{u}_{1}\left(\zeta_{1}, \zeta_{2}\right)}{\partial \bar{x}_{1}}
$$

É importante observar que a direção $\bar{x}_{1}$ é a direção tangencial no ponto onde se deseja calcular as tensões e $\bar{x}_{2}$ é a direção normal. Determinada a componente de deformação da direção $\bar{x}_{1}$, pode então, manipulando-se as relações constitutivas 
anisotrópicas (Equações 3.23 - 3.28), se determinar a componente de tensão $\bar{\sigma}_{11}$. Dessa forma, têm-se as componentes de tensão, para um ponto no contorno, para o problema elastostático:

$$
\begin{aligned}
& \bar{\sigma}_{22}=\bar{p}_{2} \\
& \bar{\sigma}_{12}=\bar{p}_{1} \\
& \bar{\sigma}_{11}=E_{\bar{x}} \bar{\varepsilon}_{11}+v_{\overline{x y}} \bar{\sigma}_{22}-\eta_{\overline{x y}, \bar{x}} \bar{\sigma}_{12}
\end{aligned}
$$

Para o problema viscoplástico, o procedimento a ser empregado é basicamente o mesmo, diferindo apenas pelo fato da componente de tensão $\bar{\sigma}_{11}$ ser dependente também das deformações plástica e viscosa na direção $\bar{x}_{1}$ do elemento de contorno, conforme equacionado na expressão (5.47):

$$
\bar{\sigma}_{i j}=\bar{C}_{i j}^{k l}\left(\bar{\varepsilon}_{k l}-\bar{\varepsilon}_{k l}^{p}\right)+\gamma \bar{C}_{i j}^{k l} \overline{\dot{\varepsilon}}_{k l}
$$

onde $\bar{C}_{i j}^{k l}$ é o tensor constitutivo anisotrópico, $\bar{\varepsilon}_{k l}^{p}$ são as deformações plásticas e $\overline{\dot{\varepsilon}}_{k l}$ a velocidade de deformação do problema viscoso, definida pela aproximação temporal linear:

$$
\overline{\dot{\varepsilon}}_{s+1}=\frac{\bar{\varepsilon}_{s+1}-\bar{\varepsilon}_{s}}{\Delta t}
$$

observa-se novamente que, todos os vetores e tensores nas Equações (5.47) e (5.48) são expressos no sistema local de coordenadas do elemento de contorno.

\subsection{TRATAMENTO ALGÉBRICO PARA AS REPRESENTAÇÕES INTEGRAIS}

Similarmente ao MEF, as equações integrais definidas anteriormente podem ser transformadas em equações algébricas através do método dos elementos de contorno. Assim, o contorno do corpo $\Gamma$ é discretizado com $n_{e}$ elementos de contorno $\Gamma_{e}$ e seu domínio $\Omega$ modelado com $n_{c}$ células $\Omega_{c}$ (Figura 5.3), de tal sorte que as densidades do contorno e do domínio sejam representadas adequadamente. Dessa forma, as variáveis 
do problema podem ser aproximadas, parametrizando-as com relação aos seus valores nodais, fazendo-se uso de funções interpoladoras apropriadas.

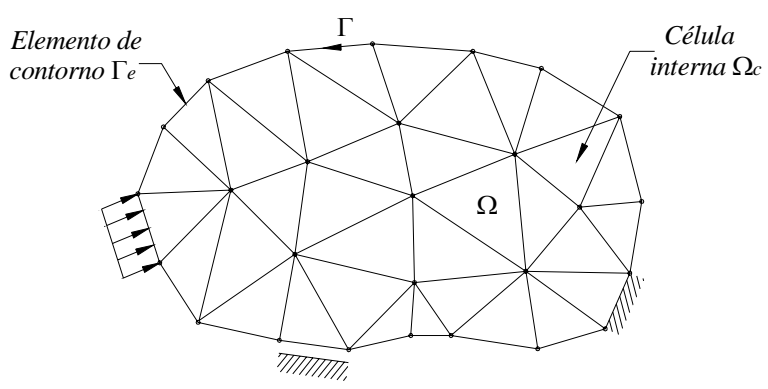

FIGURA 5.3 - Discretização do problema

$$
\begin{aligned}
& p_{i}=\phi^{\alpha} P_{i}^{\alpha} \\
& u_{i}=\phi^{\alpha} U_{i}^{\alpha} ; \quad \dot{u}_{i}=\phi^{\alpha} \dot{U}_{i}^{\alpha} \\
& \sigma_{i j}^{p}=\tilde{\phi}^{\tilde{\alpha}} \sigma_{i j}^{p(\tilde{\alpha})}
\end{aligned}
$$

onde $\phi$ e $\tilde{\phi}$ são respectivamente as funções de forma adotadas para interpolar os elementos de contorno e as células e os subscritos $\alpha$ e $\bar{\alpha}$ referem-se aos nós do elemento de contorno e da célula, respectivamente. Os termos $P_{i}^{\alpha}, \sigma_{i j}^{p(\tilde{\alpha})}, U_{i}^{\alpha}$, e $\dot{U}_{i}^{\alpha}$ são valores nodais de forças de superfície, deslocamentos, tensões residuais e velocidades. Conseqüentemente, as representações integrais de deslocamento e tensão ficam escritas como:

$$
\begin{aligned}
& \bar{C}_{k i} U_{i}(p)+\gamma \bar{C}_{k i} \dot{U}_{i}(p)=\sum_{e=1}^{n_{e}} \int_{\Gamma_{e}} u_{k i}^{*} \phi^{\alpha} d \Gamma_{e} P_{i}^{\alpha}-\sum_{e=1}^{n_{e}} \int_{\Gamma_{e}} p_{k i}^{*} \phi^{\alpha} d \Gamma_{e} U_{i}^{\alpha}+ \\
& -\gamma \sum_{e=1}^{n_{e}} \int_{\Gamma_{e}} p_{k i}^{*} \phi^{\alpha} d \Gamma_{e} \dot{U}_{i}^{\alpha}+b_{i} \sum_{e=1}^{n_{e}} \int_{\Gamma_{e}} B_{k i}^{*} d \Gamma_{e}+\sum_{c=1}^{n_{c}} \int_{\Omega_{c}} \varepsilon_{k i j}^{*} \tilde{\phi}^{\tilde{\alpha}} d \Omega_{c} \sigma_{i j}^{p(\tilde{\alpha})} \\
& \sigma_{\rho q}(p)=\sum_{e=1}^{n_{e}} \int_{\Gamma_{e}} \bar{\sigma}_{\rho q i}^{*} \phi^{\alpha} d \Gamma_{e} P_{i}^{\alpha}-\sum_{e=1}^{n_{e}} \int_{\Gamma_{e}} \bar{p}_{\rho q i}^{*} \phi^{\alpha} d \Gamma_{e} U_{i}^{\alpha}-\gamma \sum_{e=1}^{n_{e}} \int_{\Gamma_{e}} \bar{p}_{\rho q i}^{*} \phi^{\alpha} d \Gamma_{e} \dot{U} \\
& +b_{i} \sum_{e=1}^{n_{e}} \int_{\Gamma_{e}} \bar{B}_{\rho q i}^{*} d \Gamma_{e}+\sum_{c=1}^{n_{c}} \int_{\Omega_{c}} \bar{t}_{\rho q i j}^{*} \tilde{\phi}^{\widetilde{\alpha}} d \Omega_{c} \sigma_{i j}^{p(\tilde{\alpha})}+\bar{g}_{\rho q i j} \sigma_{i j}^{p}(p)
\end{aligned}
$$

Depois de executar todas as integrais e escolher pontos fonte em igual número aos nós do problema, pode-se escrever os seguintes sistemas de equações diferenciais temporais: 


$$
\begin{aligned}
& H U(t)+\gamma H \dot{U}(t)=G P(t)+B b(t)+Q \sigma^{p}(t) \\
& \sigma(t)=\tilde{G} P(t)-\tilde{H} U(t)-\gamma \tilde{H} \dot{U}(t)+\widetilde{B} b(t)+\tilde{Q} \sigma^{p}(t)
\end{aligned}
$$

onde $t$ representa o tempo. Ressalta-se que a matriz $H$ é obtida através da associação do temo $\bar{C}_{k i}$ com a matriz referente a integral de contorno cuja o núcleo é $p_{k i}^{*}$, como usualmente é feito no método dos elementos de contorno. Já o temo livre é associado a última integral em (5.51) dando origem a matriz $\widetilde{Q}$.

\subsubsection{Integração temporal numérica}

Para se resolver o problema viscoplástico, deve-se integrar no tempo o sistema de equações apresentado na Equação (5.52). Para isto, adotou-se uma simples aproximação linear para definir a velocidade de deslocamento, de maneira que:

$$
\dot{U}_{t+\Delta t}=\frac{U_{t+\Delta t}-U_{t}}{\Delta t}
$$

Aplicando-se a expressão de velocidade apresentada em (5.54) na Equação (5.52), encontra-se o seguinte sistema de equações:

$$
\hat{H} U_{t+\Delta t}=G P_{t+\Delta t}+F_{t+\Delta t}
$$

onde

$$
\begin{aligned}
& \hat{H}=\left(1+\frac{\gamma}{\Delta t}\right) H \\
& F_{t+\Delta t}=\frac{\gamma}{\Delta t} H U_{t}+B b_{t+\Delta t}+Q \sigma_{t+\Delta t}^{p}
\end{aligned}
$$

Impondo-se as condições de contorno, como usualmente é feito no método dos elementos de contorno, trocando-se as colunas das matrizes $\hat{H}$ e $G$, pode-se resolver o sistema linear apresentado na Equação (5.55), obtendo assim os deslocamentos e forças de superfície para o passo de tempo atual. Caso ocorra plastificação e conseqüentemente tensão residual, torna-se necessário redistribuir o resíduo de tensão inicial. Esta 
redistribuição caracteriza o processo iterativo. Este resíduo é reaplicado fazendo-se uso da Equação (5.55) escrita com valores totais (e não incrementais). A velocidade de deslocamento é obtida diretamente através da Equação (5.54). Com todas as incógnitas do problema de contorno obtidas no passo atual, encontram-se as tensões totais fazendose uso da expressão (5.53) para pontos internos.

$$
\sigma_{t+\Delta t}=\tilde{G} P_{t+\Delta t}-\tilde{H} U_{t+\Delta t}-\gamma \tilde{H} \dot{U}_{t+\Delta t}+\widetilde{B} b_{t+\Delta t}+\tilde{Q} \sigma_{t+\Delta t}^{p}
$$

Através do resultado do problema de contorno, encontram-se as tensões totais referentes aos nós do contorno conforme descrito no item (5.2). A tensão de tentativa elástica, necessária ao procedimento implícito de atualização, pode ser facilmente obtida adotando-se uma aproximação para a velocidade das tensões elásticas $\dot{\sigma}_{t+\Delta t}^{e}$ e iniciais $\dot{\sigma}_{t+\Delta t}^{p}$

$$
\begin{aligned}
& \dot{\sigma}_{t+\Delta t}^{e p}=\frac{\sigma_{t+\Delta t}^{e p}-\sigma_{t}^{e p}}{\Delta t} \\
& \dot{\sigma}_{t+\Delta t}^{p}=\frac{\sigma_{t+\Delta t}^{p}-\sigma_{t}^{p}}{\Delta t}
\end{aligned}
$$

Aplicando-se a expressão de $\dot{\sigma}_{t+\Delta t}^{e p}$ e $\dot{\sigma}_{t+\Delta t}^{p}$ apresentada em $(5.59$ - 5.60) na equação diferencial em (5.36), pode-se escrever:

$$
\sigma_{t+\Delta t}^{e p}=\left[\sigma_{t+\Delta t}+\frac{\gamma}{\Delta t} \sigma_{t}^{e p}-\gamma\left(\frac{\sigma_{t+\Delta t}^{p}-\sigma_{t}^{p}}{\Delta t}\right)\right] /\left(1+\frac{\gamma}{\Delta t}\right)
$$

As variáveis referentes ao instante passado “ $t$ ” são atualizadas apenas no final de cada incremento. Já aquelas referentes ao instante atual “ $t+\Delta t$ ” devem ser atualizadas em todas as iterações. Note que na expressão (5.61) necessita-se do valor das tensões totais que são calculadas como mencionado anteriormente.

Assim, calculadas as tensões totais e as tensões de tentativa elásticas, obtém-se as tensões elastoplásticas através de procedimentos usuais para corrigir as tensões de tentativa, de maneira que elas agora verifiquem o modelo não-linear adotado. As tensões viscosas são obtidas de uma forma simples e direta aplicando-se a relação da Equação (4.22). Não obtida a convergência repete-se o esquema sem atualizar as variáveis do instante anterior "t” caracterizando o processo iterativo. Caso contrário, atualiza-se as varáveis do problema e parte-se para um novo passo de tempo. 


\section{ALGORITMO PARA ATUALIZAÇÃO DAS TENSÕES - CRITÉRIO DE TSAI-WU}

Neste capítulo, de forma detalhada, é apresentado o algoritmo implícito utilizado na solução do problema não linear. É adotada uma lei de fluxo plástico nãoassociativa e obtém-se uma expressão fechada para o multiplicador plástico, utilizandose a superfície de ruptura de TSAI \& WU (1971), evitando-se assim, a utilização de processos iterativos para o cálculo desse multiplicador.

\subsection{GENERALIDADES}

Para se resolver um problema onde o comportamento não linear do material tem que ser levado em consideração é necessário a utilização de procedimentos numéricos que possibilitem a integração das equações constitutivas que regem o comportamento do material. Algoritmos eficientes são aqueles que possibilitam uma precisa integração das relações constitutivas e uma adequada velocidade de convergência, ORTIZ \& POPOV(1985), FEENSTRA \& BORST(1996) e MESQUITA(1998).

Nas análises numéricas utilizando-se os algoritmos do tipo implícito, observase que o custo computacional maior está na geração da matriz de rigidez global da estrutura (quando o método requer atualização) e no processo de atualização das tensões. O elevado custo na atualização das tensões, principalmente quando o nível de plastificação é alto, é devido ao procedimento iterativo utilizado para se resolver a 
equação de consistência e se obter o multiplicador plástico (MESQUITA, 2002). Entretanto, o multiplicador plástico pode ser obtido através de simples expressões explícitas encontradas através de uma adequada manipulação das relações constitutivas do modelo proposto, sem o uso de procedimentos iterativos, conforme realizado por MESQUITA (2002), o qual, desenvolveu expressões para este multiplicador, caso tridimensional, para os critérios de von Mises e Drucker-Prager com encruamento isotrópico e leis de fluxo associativas e não-associativas. No presente trabalho, a expressão do multiplicador plástico é também obtida de forma explícita.

Para tanto, neste item, o algoritmo do tipo implícito utilizado para atualização das tensões é apresentado e a expressão fechada do multiplicador plástico a ser utilizado é obtida para o caso bidimensional. O critério de resistência utilizado, como já comentado, é o de TSAI \& WU (1971) com encruamento isotrópico e lei de fluxo nãoassociativa. Esta abordagem, não-associativa, segue a suposição de que o fluxo plástico tende a seguir uma direção cujo seu produto interno com a direção do fluxo elástico seja positiva, garantindo assim, retorno à superfície de plastificação.

\subsection{CONSIDERAÇÕES GERAIS SOBRE PLASTICIDADE}

Em estados multiaxiais de tensão a região elástica é limitada no espaço das tensões principais por uma superfície conhecida como superfície de plastificação, a qual separa os estados de tensão elásticos dos plásticos, que geram deformações irreversíveis. Assim, a plastificação em um ponto fica caracterizada pela verificação da igualdade na relação matemática que define a superfície de plastificação, ou seja:

$$
f\left(\sigma_{i j}, \kappa\right)=\bar{f}\left(\sigma_{i j}\right)-\bar{\sigma}(\kappa)=0
$$

onde $\bar{\sigma}$ é uma tensão equivalente que é função do parâmetro de encruamento $\kappa$, e $\bar{f}$ é uma função do estado de tensão $\sigma_{i j}$. Assume-se que as tensões limites só serão alcançadas se a relação matemática que representa a superfície for satisfeita $(f=0)$. Partindo-se desta hipótese, um descarregamento (retorno à região elástica) fica evidenciado por variações no estado de tensão que conduzam a valores negativos de $f$.

A situação limite de carregamento neutro é caracterizada por acréscimos no estado de tensão que conduzam a verificação da Equação (6.1) com a igualdade. 
Alterações no estado de tensão que determinem valores positivos de $f$ são inacessíveis e indicam o aparecimento de deformações irreversíveis, caracterizando uma situação de carregamento plástico. Nesta última situação, de acordo com o modelo de encruamento do material, a superfície de plastificação tende a evoluir, de forma que o novo estado de tensão ainda resulte sobre a mesma.

Neste contexto, abordando-se o problema não linear em taxas, a deformação total pode ser decomposta em uma parcela elástica reversível $\left(\dot{\varepsilon}_{i j}^{e}\right)$ e uma parcela plástica irreversível $\left(\dot{\varepsilon}_{i j}^{p}\right)$.

$$
\dot{\varepsilon}_{i j}=\dot{\varepsilon}_{i j}^{e}+\dot{\varepsilon}_{i j}^{p}
$$

A taxa de deformação elástica é relacionada com a taxa de tensão através da matriz constitutiva elástica $C_{i j l m}^{e}$, da seguinte forma:

$$
\dot{\sigma}_{i j}=C_{i j l m}^{e} \dot{\varepsilon}_{l m}^{e}
$$

Nas formulações usuais, a taxa de deformação plástica é expressa através da seguinte relação:

$$
\dot{\varepsilon}_{i j}^{p}=\dot{\lambda} \frac{\partial g}{\partial \sigma_{i j}}=\dot{\lambda} \partial_{\sigma} g_{i j}
$$

onde $\dot{\lambda}$ é uma constante de proporcionalidade denominada multiplicador plástico, que deve satisfazer as seguintes condições de complementaridade de Kuhn-Tucker,

$$
\dot{\lambda} \geq 0, \quad f \leq 0 \quad \text { e } \dot{\lambda} \cdot f=0
$$

O termo $g$ na equação (6.4) é um potencial plástico com unidade de tensão. O caso particular de $g=f$ é conhecido como plasticidade associativa. Uma outra forma de idealizar o fluxo plástico seria através da seguinte relação:

$$
\dot{\varepsilon}_{i j}^{p}=\dot{\lambda} n_{i j}
$$

onde $n_{i j}$ é um tensor de módulo unitário que define a direção do fluxo plástico. A direção adotada $n_{i j}$ não é necessariamente normal à superfície de plastificação no espaço das tensões principais, caracterizando lei de fluxo associativa. Para o caso de 
encruamento por deformação o escalar $\dot{\kappa}$ é relacionado com a norma do tensor das taxas de deformações plásticas:

$$
\dot{\kappa}=\dot{\varepsilon}^{e q}=K\left\|\dot{\varepsilon}_{i j}^{p}\right\| \Rightarrow \kappa=\varepsilon^{e q}=\int K\left\|\dot{\varepsilon}_{i j}^{p}\right\|
$$

onde $\dot{\varepsilon}_{i j}^{p}$ é o tensor das taxas de deformações plásticas, o escalar $\varepsilon^{e q}$ é conhecido como deformação plástica equivalente e $K$ é um termo cujo valor deve ser escolhido de tal forma que, a partir do modelo generalizado, possa-se recuperar o caso unidimensional.

\subsection{ASPECTOS INCREMENTAIS DO ALGORITMO}

A integração das equações em taxa é um problema de evolução. As variáveis de estado em um certo instante $i$ são conhecidas e devem ser atualizadas de uma forma consistente, determinando assim, o estado atual referente ao instante $i+1$. As variáveis internas são determinadas através da utilização de um procedimento implícito (Backward Euler), caindo em um problema governado pelas condições de Kuhn-Tucker, expressas em forma incremental por:

$$
\Delta \lambda_{i+1} \geq 0, \quad f_{i+1} \leq 0 \quad \text { e } \Delta \lambda_{i+1} f_{i+1}=0
$$

Desta forma, as equações do algoritmo também podem ser expressas incrementalmente como:

$$
\begin{aligned}
& \varepsilon_{i+1}=\varepsilon_{i}+\Delta \varepsilon_{i+1} \\
& \sigma_{i+1}=C^{e}\left(\varepsilon_{i+1}-\varepsilon_{i+1}^{p}\right) \\
& \varepsilon_{i+1}^{p}=\varepsilon_{i}^{p}+\Delta \varepsilon_{i+1}^{p} \\
& \kappa_{i+1}=\kappa_{i}+\Delta \kappa_{i+1}
\end{aligned}
$$

No processo de integração das tensões pelo procedimento implícito, é necessário considerar um estado de tentativa (previsão), que é tomado como um passo puramente elástico, através das seguintes relações:

$$
\begin{aligned}
& \varepsilon_{i+1}=\varepsilon_{i}+\Delta \varepsilon_{i+1} \\
& \sigma_{i+1}^{t e n}=C^{e}\left(\varepsilon_{i+1}-\varepsilon_{i}^{p}\right) \\
& \kappa_{i+1}^{t e n}=\kappa_{i} \\
& f\left(\sigma_{i+1}^{t e n}, \kappa_{i+1}^{t e n}\right)=\bar{f}\left(\sigma_{i+1}^{t e n}\right)-\bar{\sigma}\left(\kappa_{i+1}^{t e n}\right)
\end{aligned}
$$


A partir deste estado de tentativa é possível determinar se o estado de tensão viola o critério definido pela superfície de plastificação. Se o estado de tensão obtido na previsão elástica não violar o critério, significa que o estado obtido é admissível e compatível com o modelo adotado. Porém, se este violar, um outro estado de tensão deve ser procurado de modo a se tornar compatível com o modelo. Assim, tem-se:

$$
\begin{aligned}
& \sigma_{i+1}=\sigma_{i+1}^{t e n}-\Delta \lambda_{i+1} C^{e} n_{i+1} \\
& \varepsilon_{i+1}^{p}=\varepsilon_{i}^{p}+\Delta \lambda_{i+1} n_{i+1} \\
& \kappa_{i+1}=\kappa_{i}+\Delta \kappa_{i+1}\left(\Delta \lambda_{i+1}\right)
\end{aligned}
$$

Deve-se observar que as expressões $(6.17$ - 6.19) dependem da determinação do multiplicador plástico $\Delta \lambda$ no instante atual. Sendo assim, obtendo-se o escalar $\Delta \lambda_{i+1}$ é possível encontrar as variáveis internas em $(6.17$ - 6.19) e resolver o problema no instante $i+1$. O multiplicador plástico pode ser encontrado resolvendo-se a condição de consistência no instante $i+1$, ou seja:

$$
f\left(\sigma_{i+1}, \kappa_{i+1}\right)=\bar{f}\left(\sigma_{i+1}\right)-\bar{\sigma}\left(\kappa_{i+1}\right)=0
$$

Substituindo-se as expressões de atualização do encruamento $\kappa_{i+1}$, Equação (6.19), e das tensões $\sigma_{i+1}$ na condição de consistência (6.20) encontra-se uma nova equação escrita em função apenas do multiplicador plástico $\Delta \lambda_{i+1}$. Esta equação deve ser resolvida, determinando-se o $\Delta \lambda$ que recupera um novo estado compatível com o modelo adotado. No item seguinte, apresenta-se a determinação do multiplicador plástico $\Delta \lambda$, considerando-se o critério de TSAI \& WU (1971) e o modelo de encruamento isotrópico para o caso bidimensional, com lei de fluxo não associativa.

\subsection{ALGORITMO TSAI \& WU (1971) NÃO ASSOCIATIVO}

O critério de plastificação utilizando-se a superfície de TSAI \& WU (1971) pode ser escrito como:

$$
f=\bar{f}(\sigma)-\bar{\sigma}(\kappa)=\sigma^{T} F \sigma+L \sigma-\bar{\sigma}(\kappa) \leq 0
$$

onde o termo $F$ é uma matriz simétrica composta pelos valores limites de tensão de escoamento do material, definidos no item 3.4.1, e que para o caso plano tem a forma: 


$$
F=\left[\begin{array}{lll}
F_{11} & F_{12} & F_{16} \\
& F_{22} & F_{26} \\
\operatorname{sim} & & F_{66}
\end{array}\right]
$$

sendo o termo $L$ um vetor que caracteriza a diferença entre as tensões de tração e compressão, nas duas direções do material, e que também é dependente dos valores limites de tensão de escoamento do material. O vetor $L$ é definido da seguinte forma:

$$
L=\left\{\begin{array}{l}
F_{1} \\
F_{2} \\
F_{6}
\end{array}\right\}
$$

Já $\sigma$ é o tensor de tensões escrito em forma vetorial como:

$$
\sigma^{T}=\left\{\begin{array}{lll}
\sigma_{11} & \sigma_{22} & \sigma_{12}
\end{array}\right\}
$$

A tensão equivalente $\bar{\sigma}$ é definida, neste trabalho, segundo uma lei de encruamento isotrópica linear (Figura 6.1) escrita em função de parâmetros de plastificação e do encruamento isotrópico $\kappa$ e seu respectivo módulo plástico $H$.

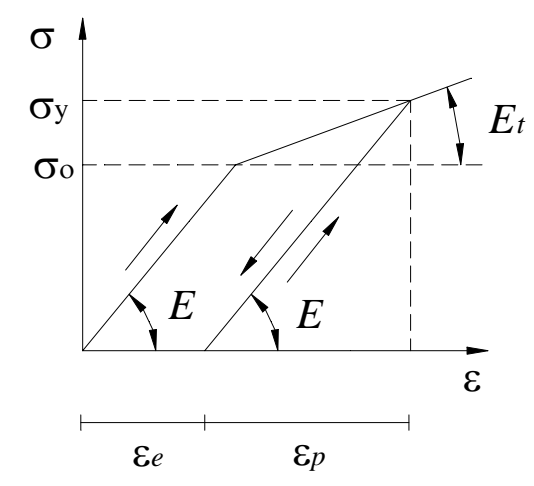

FIGURA 6.1 - Lei de encruamento

$$
\bar{\sigma}(\kappa)=H^{2} \kappa^{2}
$$

onde o valor inicial de $\kappa$ deve ser igual a $1 / H$ para que se possa, na consideração do encruamento, se obter uma expressão compatível com a Equação (3.85) do critério, item 3.4.1. Deve-se observar que a expressão quadrática (6.25) representa crescimento linear em relação à superfície de plastificação definida na Equação (6.21), também representada por uma forma quadrática. 
As deformações plásticas incrementais são definidas segundo uma lei de fluxo não associativa. Esta idealização parte da suposição de que o sólido ao se plastificar poderá se comportar como um fluido compressivo, ou seja, não se pode condicionar o fluxo plástico a uma superfície responsável, em princípio, por definir apenas os limites elásticos do material.

Assim, imagina-se que se deve estabelecer a direção do fluxo plástico mantendo-se uma relação com as tensões, sendo esta relação semelhante à relação elástica (tensão/deformação). Neste sentido, pode-se escolher um tensor unitário qualquer $n$ como sendo a direção do fluxo plástico:

$$
\Delta \varepsilon^{p}=\Delta \lambda \cdot n=\Delta \lambda \varepsilon^{t e n}=\Delta \lambda C^{p-1} \sigma^{t e n}
$$

onde $C^{p-1}$ é um tensor semelhante ao tensor constitutivo elástico $C^{e}$.

Para o caso associativo, como feito por MESQUITA (2002) para os critérios de von Mises e Drucker Praguer, a expressão para o incremento de deformação plástica para o critério de TSAI \& WU (1971) assume a seguinte forma:

$$
\Delta \varepsilon^{p}=\Delta \lambda \frac{\partial f}{\partial \sigma}=\Delta \lambda \partial_{\sigma} f=\Delta \lambda(F \sigma+L)
$$

A particularização da expressão (6.26) adotando-se no lugar da matriz constitutiva plástica $C^{p}$ a matriz elástica $C^{e}$, faz com que o fluxo plástico tenha seu retorno dado na direção das deformações elásticas aplicadas ao corpo. Com a utilização da forma geral, esta direção de retorno pode ser qualquer dependendo de quão compressível seja o material em estudo na fase plástica. Para se obter material incompressível na fase plástica adota-se $C^{p}$ semelhante a uma lei constitutiva isotrópica com “coeficiente de Poisson plástico” $\left(v_{p}\right)$ igual a meio. Dessa forma, poderíamos quantificar qualquer grau de compressibilidade do material plastificado. Assim, a expressão de atualização das deformações plásticas fica escrita como:

$$
\varepsilon_{i+1}^{p}=\varepsilon_{i}^{p}+\Delta \varepsilon_{i+1}^{p}=\varepsilon_{i}^{p}+\Delta \lambda_{i+1} C^{p^{-1}} \sigma_{i+1}^{t e n}
$$

O incremento $\Delta \kappa$ é obtido considerando-se hipótese de encruamento por deformação. Para tanto tem-se; 


$$
\Delta \kappa^{2}=\Delta \varepsilon^{e q}=K\left\|\Delta \varepsilon_{i j}^{p}\right\|=K \Delta \lambda^{2}\left(\left(C^{p^{-1}} \sigma_{i+1}^{t e n}\right)^{T} F\left(C^{p^{-1}} \sigma_{i+1}^{t e n}\right)\right)
$$

onde $K$ tem unidade de tensão e deve ser determinado para que a expressão permita a recuperação do caso unidimensional. Como a degeneração do problema anisotrópico geral ficaria complicada usando a norma indicada em (6.29), propõe-se a seguinte norma para simplificação das expressões:

$$
\Delta \kappa^{2}=\Delta \varepsilon^{e q}=K\left\|\Delta \varepsilon_{i j}^{p}\right\|=K \Delta \lambda^{2}\left(\sigma^{t e n^{T}} F \sigma^{t e n}\right)
$$

Deve-se recuperar o fator de escala para se compor o parâmetro de encruamento $k$ como segue, onde o coeficiente $K$ deve ser determinado para esta nova situação, guardando-se ainda a necessidade de se retornar para o caso unidimensional.

Assim, a expressão de atualização do parâmetro de encruamento fica:

$$
\kappa_{i+1}=\kappa_{i}+\Delta \kappa_{i+1}=\kappa_{i}+\Delta \lambda_{i+1} \sqrt{K \sigma_{i+1}^{t e n^{T}} F \sigma_{i+1}^{\text {ten }}}
$$

Retornando à parte da expressão da superfície de plastificação referente à evolução do critério tem-se:

$$
\bar{\sigma}(\kappa)=H^{2} \kappa_{i+1}^{2}=H^{2}\left(\kappa_{i}^{2}+\Delta \lambda_{i+1}^{2} K \sigma_{i+1}^{t e n} F \sigma_{i+1}^{t e n}+2 \kappa_{i} \Delta \lambda_{i+1} \sqrt{K \sigma_{i+1}^{t e n} F \sigma_{i+1}^{t e n}}\right)
$$

Da expressão (6.32) entende-se que $K$ deve ser de dimensão da inversa da tensão ao quadrado. Para que o critério degenere no caso unidimensional (direção ortotrópica 1) $K$ deve assumir o seguinte valor:

$$
K=\frac{1}{E_{1}^{2}}
$$

considerando-se portanto degeneração na direção 1 com módulo de elasticidade longitudinal $E_{1}$.

Para a expressão de atualização das tensões tem-se:

$$
\sigma_{i+1}=\sigma_{i}+\Delta \sigma_{i+1}=\sigma_{i}+C^{e}\left(\Delta \varepsilon_{i+1}-\Delta \varepsilon_{i+1}^{p}\right)
$$


Definindo-se um estado de tentativa elástico (ou seja, $\Delta \varepsilon_{i+1}^{p}=0$ ) é possível escrever a equação (6.34) como:

$$
\sigma_{i+1}=\sigma_{i}+\Delta \sigma_{i+1}=\sigma_{i+1}^{t e n}-C^{e} \Delta \varepsilon_{i+1}^{p}
$$

Substituindo-se a expressão de $\Delta \varepsilon_{i+1}^{p}$ dada pela Equação (6.28) na expressão de atualização das tensões (6.34), encontra-se:

$$
\sigma_{i+1}=\sigma_{i+1}^{t e n}-C^{e} \Delta \lambda_{i+1} C^{P^{-1}} \sigma_{i+1}^{t e n}=\left(1-\Delta \lambda_{i+1} C^{e} C^{P^{-1}}\right) \sigma_{i+1}^{t e n}
$$

Analisando-se a equação de atualização das tensões em (6.36) é possível visualizar que o retorno do estado de tentativa (Figura 6.2) não provoca o aparecimento de tensões residuais em direções diferentes da estabelecida pela tentativa elástica quando se escolhe $C^{p}=C^{e}$.

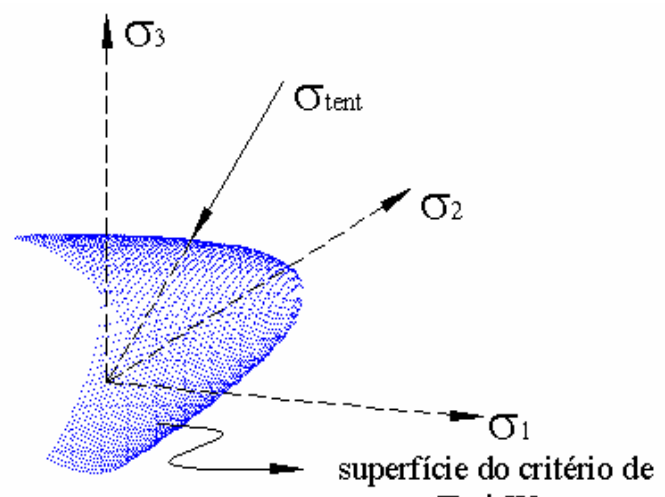

Tsai-Wu

FIGURA 6.2 - Superfície do critério de TSAI \& WU (1971) e a direção das tensões tentativas.

Agora deve-se encontrar a expressão da condição de consistência de maneira que se possa resolve-la e assim se determinar o multiplicador plástico. Para o instante atual o critério de plastificação fica escrito como:

$$
f=\sigma^{T} F \sigma+L \sigma-\bar{\sigma}(\kappa)=0
$$

Desenvolvendo-se os dois primeiros termos, do segundo membro, da Equação (6.37) e fazendo-se uso da expressão de atualização das tensões em (6.36), tem-se:

$$
\begin{aligned}
\left(\sigma^{T} F \sigma+L \sigma\right)= & \left(1-\Delta \lambda_{i+1} C^{e} C^{p^{-1}}\right)^{T} \sigma_{i+1}^{t e n} \cdot F \cdot\left(1-\Delta \lambda_{i+1} C^{e} C^{p^{-1}}\right) \sigma_{i+1}^{t e n}+ \\
& +L \cdot\left(1-\Delta \lambda_{i+1} C^{e} C^{p^{-1}}\right) \sigma_{i+1}^{t e n}
\end{aligned}
$$


Aplicando-se a definição de encruamento expressa pela Equação (6.31), o terceiro termo, do segundo membro, da Equação (6.37) fica escrita como:

$$
\left.\bar{\sigma}\left(\kappa_{i+1}\right)=H^{2} \kappa_{i}^{2}+H^{2} \Delta \lambda^{2} K \cdot\left(\sigma_{i+1}^{\text {ten }}{ }^{T} F \sigma_{i+1}^{t e n}\right)+2 H^{2} \kappa_{i} \cdot \Delta \lambda \sqrt{K \cdot\left(\sigma_{i+1}^{t e n} T F \sigma_{i+1}^{t e n}\right.}\right)
$$

Substituindo as expressões (6.38) e (6.39) na expressão do critério de plastificação em (6.37), encontra-se:

$$
\begin{gathered}
f=\left(1-\Delta \lambda_{i+1} C^{e} C^{p^{-1}}\right)^{T} \sigma_{i+1}^{t e n} \cdot F \cdot\left(1-\Delta \lambda_{i+1} C^{e} C^{p^{-1}}\right) \sigma_{i+1}^{\text {ten }}+ \\
+L \cdot\left(1-\Delta \lambda_{i+1} C^{e} C^{p^{-1}}\right) \sigma_{i+1}^{t e n}-H^{2} \kappa_{i}^{2}-H^{2} \Delta \lambda^{2} K \cdot\left(\sigma_{i+1}^{t e n} F \sigma_{i+1}^{t e n}\right)+ \\
\left.-2 H^{2} \kappa_{i} \cdot \Delta \lambda \sqrt{K \cdot\left(\sigma_{i+1}^{t e n} T \sigma_{i+1}^{t e n}\right.}\right)=0
\end{gathered}
$$

Assim, organizando-se a Equação (6.40) para $\Delta \lambda$, chamando-se também de $\bar{A}$ a multiplicação de matrizes $C^{e} C^{p^{-1}}$, obtém-se uma equação de segundo grau que pode ser escrita como:

$$
f=\alpha \Delta \lambda^{2}+\beta \Delta \lambda+\gamma=0
$$

onde:

$$
\begin{aligned}
& \alpha=\sigma_{i+1}^{t e n} T \bar{A} \cdot F \cdot \bar{A} \sigma_{i+1}^{t e n}-H^{2} K \cdot\left(\sigma_{i+1}^{t e n} T F \sigma_{i+1}^{t e n}\right) \\
& \left.\beta=-\sigma_{i+1}^{t e n} T \bar{A} \cdot F \sigma_{i+1}^{t e n}-\sigma_{i+1}^{t e n} F \cdot \bar{A} \sigma_{i+1}^{t e n}-L \cdot \bar{A} \sigma_{i+1}^{t e n}-2 H^{2} \kappa_{i} \sqrt{K \cdot\left(\sigma_{i+1}^{t e n} T\right.} F \sigma_{i+1}^{t e n}\right) \\
& \gamma=\sigma_{i+1}^{t e n} T F \sigma_{i+1}^{t e n}+L \sigma_{i+1}^{t e n}-H^{2} \kappa_{i}^{2}
\end{aligned}
$$

Com isso, resolvendo a Equação (6.41) adota-se, como multiplicador plástico $\Delta \lambda$, o menor valor positivo entre as suas duas raízes. Observa-se que dessa maneira o multiplicador plástico $\Delta \lambda$ é obtido de forma direta sem a necessidade de se empregar qualquer processo iterativo. As duas expressões das raízes da Equação (6.41) podem ser consideradas como duas expressões fechadas para a determinação do multiplicador plástico $\Delta \lambda$. Se o valor de $\alpha$ for nulo, então a Equação (6.41) recai numa expressão de $1^{\circ}$ grau e o valor do multiplicador plástico $\Delta \lambda$ é único para a expressão. 
De posse do multiplicador plástico $\Delta \lambda$, as variáveis internas do processo incremental-iterativo, tensões, deformações e encruamento podem ser atualizadas conferindo-se assim, consistência à análise não-linear física conforme pode ser constatado no esquema geral apresentado na Figura 6.3:

1. Atualizar as deformações e calcular as tensões e parâmetro de encruamento de tentativa

$$
\begin{aligned}
& \varepsilon_{i+1}=\varepsilon_{i}+\Delta \varepsilon_{i+1} \\
& \sigma_{i+1}^{t e n}=C^{e}\left(\varepsilon_{i+1}-\varepsilon_{i}^{p}\right) \\
& \kappa_{i+1}^{t e n}=\kappa_{i}
\end{aligned}
$$

2. Verificar o critério de plastificação com as tensões de tentativa

$$
f_{i+1}^{t e n}=\sigma_{i+1}^{t e n^{T}} F \sigma_{i+1}^{t e n}+\sigma_{i+1}^{t e n} L-\bar{\sigma}(\kappa)
$$

3. Se $f_{i+1}^{\text {ten }} \leq 0$

\subsection{Então:}

\subsubsection{Finalizar}

3.2 Se não:

\subsubsection{Determinar $\Delta \lambda_{i+1}$}

$n_{i+1}=C^{p^{-1}} \sigma_{i+1}^{t e n}$

$K=\frac{1}{E_{1}^{2}}$ para degeneração na direção 1.

$\Delta \lambda_{i+1}=\frac{-\beta \pm \sqrt{\beta^{2}-4 \alpha \gamma}}{2 \alpha}$ adotado-se o menor valor positivo.

3.2.2 Atualizar as variáveis internas com o valor de $\Delta \lambda_{i+1}$

$$
\begin{aligned}
& \sigma_{i+1}=\left(1-C^{e} \Delta \lambda_{i+1} C^{p^{-1}}\right) \sigma_{i+1}^{t e n} \\
& \varepsilon_{i+1}^{p}=\varepsilon_{i}^{p}+\Delta \lambda_{i+1} n_{i+1} \\
& \kappa_{i+1}=\kappa_{i}+\Delta \lambda_{i+1} \sqrt{K\left(\sigma_{i+1}^{\text {ten }} F \sigma_{i+1}^{t e n}\right)}
\end{aligned}
$$

FIGURA 6.3 - Esquema geral de análise no processo não-linear.

Descrito o algoritmo implícito utilizado nas análises não lineares físicas desta pesquisa, apresenta-se então, nos próximos capítulos, alguns exemplos de aplicações dos códigos computacionais desenvolvidos fazendo-se uso das formulações propostas. 


\section{APLICAÇõES - ANÁLISE ELÁSTICA LINEAR}

Neste capítulo são apresentados três exemplos onde a formulação elástica linear para meios anisotrópicos é aplicada. Os dois primeiros são analisados através do MEF e do MEC e as soluções, em termos de tensões, são confrontadas por meio de diagramas comparativos, permitindo-se verificar o comportamento das duas aproximações. Como comentado anteriormente, na análise pelo MEF dois elementos finitos são utilizados, os elementos triangulares LST e QST (Figura 4.5). Para o MEC, são utilizados elementos de contorno retos com aproximação quadrática.

No desenvolvimento dos dois primeiros exemplos são consideradas duas vigas laminadas, assumidas homogêneas e anisotrópicas, cujas lâminas são constituídas por um material polimérico reforçado com fibras de carbono. As propriedades elásticas das lâminas consideradas estão apresentadas na Tabela 7.1 (HYER, 1997):

TABELA 7.1 - Constantes elásticas do material reforçado que constitui as lâminas consideradas

\begin{tabular}{ccc}
\multicolumn{3}{c}{- eixos locais. } \\
\hline Constantes elásticas & Nomenclatura & Valor (GPa) \\
\hline Módulo de Young na direção local 1 & $\mathrm{E}_{1}$ & 155,00 \\
Módulo de Young na direção local 2 & $\mathrm{E}_{2}$ & 12,10 \\
Módulo de elasticidade transversal & $\mathrm{G}_{12}$ & 4,40 \\
Coeficiente de Poisson & $v_{12}$ & 0,25
\end{tabular}

Salienta-se que essas propriedades elásticas estão referidas às direções locais das lâminas, ou seja, às direções das fibras de reforço dispostas com um ângulo $(\theta)$ em relação ao eixo global $x$ da estrutura, Figura 7.1: 


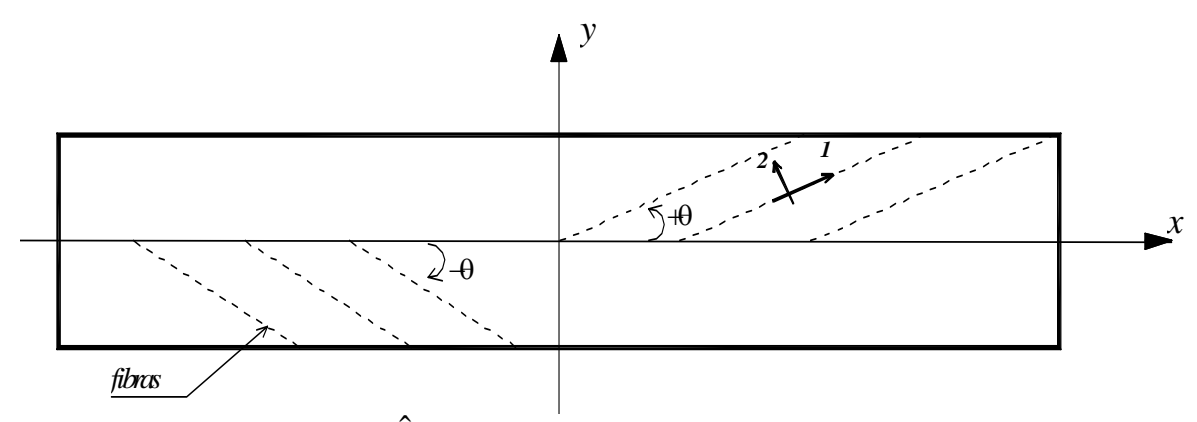

FIGURA 7.1 - Ângulo de orientação das fibras nas lâminas.

Com isso, pode-se transformar as propriedades de cada lâmina para o sistema global de coordenadas onde a estrutura está referida, utilizando-se matrizes de transformação de propriedades elásticas. Fazendo-se isso para cada lâmina constituinte da estrutura, é possível somar os tensores constitutivos de todas as lâminas, compondose então, um tensor constitutivo global anisotrópico para a viga laminada considerada então, homogênea e anisotrópica.

Deve-se comentar que como cada lâmina tem sua espessura específica, na composição do tensor constitutivo global, isso deve ser levado em conta fazendo-se uma soma ponderada das características elásticas de cada lâmina, como mostra a expressão (7.1) apropriada para a análise de chapas:

$$
\left(\bar{S}_{i j}\right)_{\text {global }}=\frac{\sum_{k=1}^{\text {ncam }} \bar{S}_{i j}{ }^{k} \cdot e_{k}}{\sum_{k=1}^{\text {ncam }} e_{k}}
$$

onde:

$$
\begin{aligned}
& \left(\bar{S}_{i j}\right)_{\text {global }} \quad \text { São as constantes do tensor constitutivo global da estrutura; } \\
& \bar{S}_{i j}^{k} \quad: \text { São as constantes do tensor constitutivo de cada camada; } \\
& e_{k} \quad: \text { É a espessura de cada camada. }
\end{aligned}
$$

Assim, em ambos exemplos, considerou-se as lâminas dispostas com uma orientação de 30 graus $\left(\theta=+30^{\circ}\right)$ em relação ao eixo global x. Com isso, a partir das propriedades apresentadas na Tabela 7.1, têm-se as seguintes propriedades elásticas anisotrópicas referidas às direções globais das vigas: 
TABELA 7.2 - Constantes elásticas do material reforçado que constitui as lâminas consideradas - eixos globais.

\begin{tabular}{ccc}
\hline Constantes Elásticas & Nomenclatura & Valor (GPa) \\
\hline Módulo de Young na direção global x & $\mathrm{E}_{\mathrm{x}}$ & 19,681 \\
Módulo de Young na direção global y & $\mathrm{E}_{\mathrm{y}}$ & 11,248 \\
Módulo de elasticidade transversal & $\mathrm{G}_{\mathrm{xy}}$ & 7,933 \\
Coeficiente de Poisson & $v_{\mathrm{xy}}$ & 0,529 \\
Coeficiente de influência mútua (1 ${ }^{\mathrm{a}}$.espécie) & $\eta_{\mathrm{xy}, \mathrm{x}}$ & $-1,224$ \\
Coeficiente de influência mútua (2 ${ }^{\mathrm{a}}$.espécie) & $\eta_{\mathrm{xy}, \mathrm{y}}$ & $-0,042$ \\
\hline
\end{tabular}

No terceiro exemplo, onde somente o programa desenvolvido em elementos de contorno é aplicado, um procedimento alternativo para a determinação dos fatores de intensidade de tensão (KI e KII) em problemas de mecânica da fratura é proposto (VANALLI et al., 2003b). Salienta-se que aplicações no campo da mecânica fratura não estavam previstas nos objetivos iniciais do trabalho e que, a idéia proposta, surgiu de observações das soluções numéricas feitas no decorrer da presente pesquisa, para os problemas elásticos, como, por exemplo, a observação do bom comportamento das soluções nos problemas quase singulares, onde o ponto fonte é considerado muito próximo ao contorno do corpo.

\subsection{EXEMPLO 1 - VIGA ENGASTADA}

O primeiro exemplo é a viga engastada apresentada na Figura 7.2 com suas características geométricas e carregamento uniformemente distribuído de $10 \mathrm{kN} / \mathrm{m}$ :

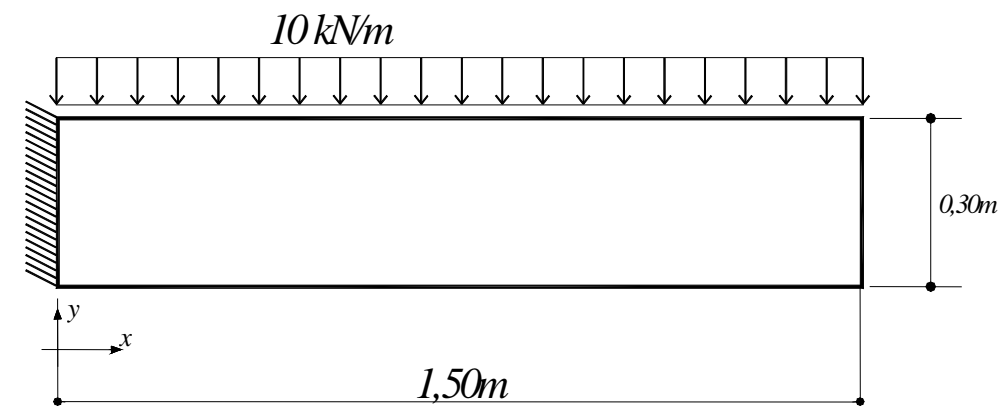

FIGURA 7.2 - Viga engastada submetida a um carregamento uniforme 
As soluções numéricas são comparadas com os resultados analíticos de LEKHNITSKII et al. (1968) para uma viga anisotrópica submetida às mesmas condições de contorno. Salienta-se que esses resultados analíticos são também aproximados, pois LEKHNITSKII et al. (1968) fazem uso de funções de tensão polinomiais (Funções de Airy) e essas funções não podem representar exatamente as condições de contorno do problema. É importante mencionar a discretização adotada, para ambos métodos:

TABELA 7.3 - Discretização adotada.

\begin{tabular}{ccc}
\hline Método & Elementos $(\boldsymbol{x}-\boldsymbol{y})$ & $\begin{array}{c}\text { Graus de } \\
\text { liberdade }\end{array}$ \\
\hline MEF / LST & $96 \times 12$ & 1261 \\
MEF / QST & $60 \times 6$ & 1820 \\
MEC & $60 \times 12$ (elementos quadráticos) & 684 \\
\hline
\end{tabular}

A seguir são apresentados os resultados de tensões obtidos para duas seções transversais, uma seção mais próxima do engaste $(\mathrm{x}=0,5 \mathrm{~m})$ e a outra no meio do vão (x $=0,75 \mathrm{~m})$.

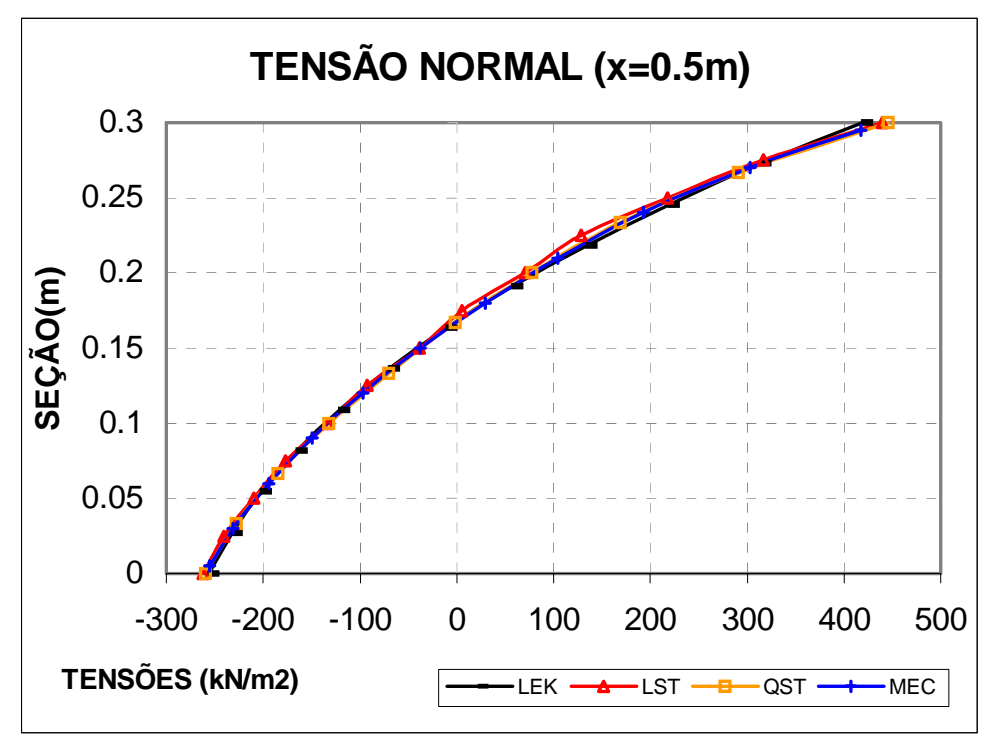

FIGURA 7.3 - Tensões normais para a seção $\mathrm{x}=0,5 \mathrm{~m}$. 


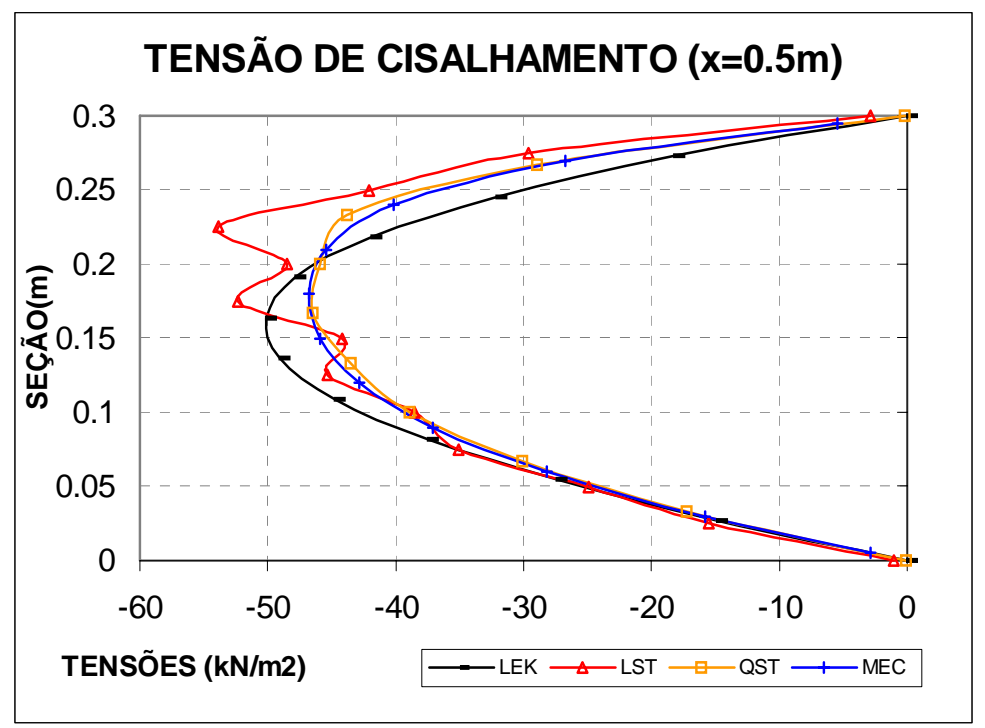

FIGURA 7.4 - Tensões de cisalhamento para a seção $\mathrm{x}=0,5 \mathrm{~m}$.

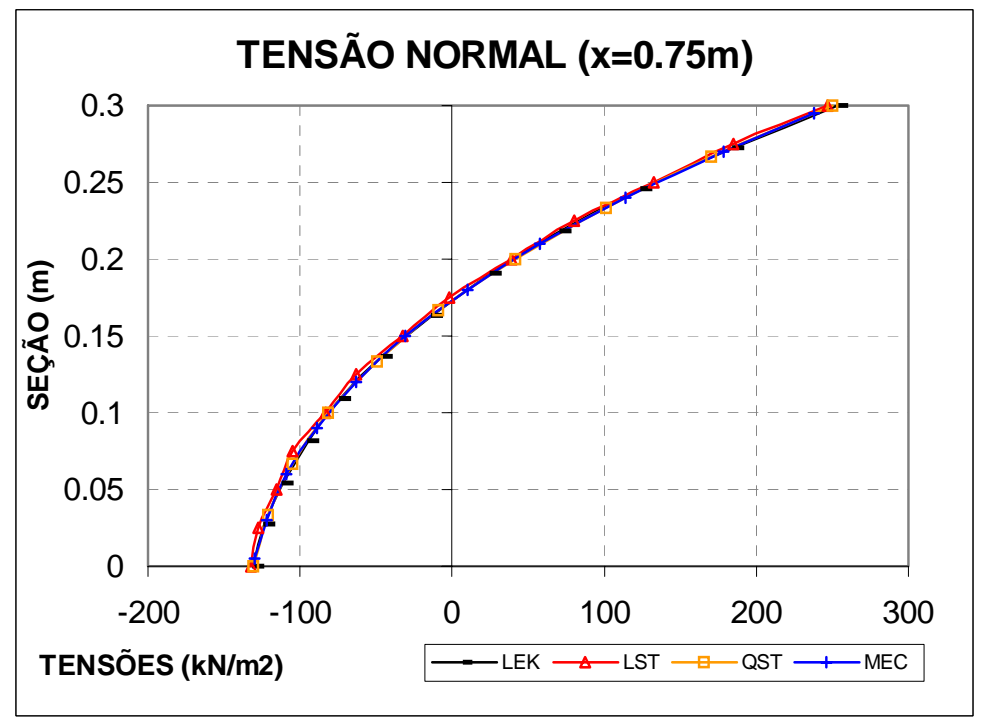

FIGURA 7.5 - Tensões normais para a seção $x=0,75 m$.

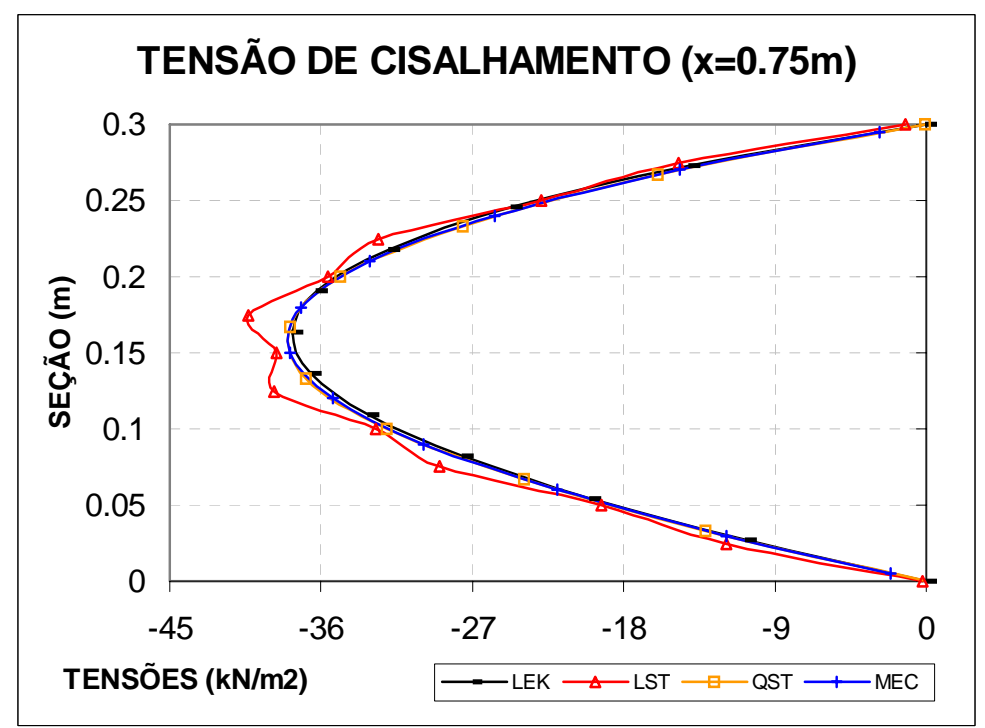

FIGURA 7.6 - Tensões de cisalhamento para a seção $\mathrm{x}=0$,75m. 
Pode-se observar nos diagramas de tensões normais, para ambas seções de análise, uma grande concordância entre os resultados analíticos aproximados de LEKHNITSKII et al. (1968) e os numéricos obtidos com o MEC e com o MEF/QST. Com relação às tensões normais oriundas do MEF/LST, percebe-se uma sensível diferença entre essas soluções e as respostas do MEC e do MEF/QST.

Nas tensões de cisalhamento as diferenças entre as duas aproximações de elementos finitos são maiores, acentuando-se na seção transversal mais próxima do apoio. Porém, os resultados do MEC e do MEF/QST estão praticamente coincidentes nas duas seções, o que ressalta a boa aproximação alcançada e a necessidade de se utilizar aproximação pelo menos quadrática para as tensões na análise de meios anisotrópicos (VANALLI et al., 2003a). Em relação ao resultado analítico próximo ao engaste, como já comentado, as funções de tensão polinomiais não podem capturar todos os efeitos presentes nos apoios (princípio de Saint Venant).

\subsection{EXEMPLO 2 - VIGA SIMPLESMENTE APOIADA COM UM FURO NO DOMÍNIO}

Analisa-se uma viga bi-apoiada anisotrópica, possuindo um furo em seu domínio, utilizando-se o MEC (aproximação quadrática) e o MEF/QST. Salienta-se que não são feitas comparações com resultados analíticos, pois não se encontrou na literatura algum trabalho que tenha abordado esse tipo de exemplo anisotrópico. A viga está apresentada na Figura 7.7 com suas características geométricas e um carregamento uniformemente distribuído de 10,0 kN/m:

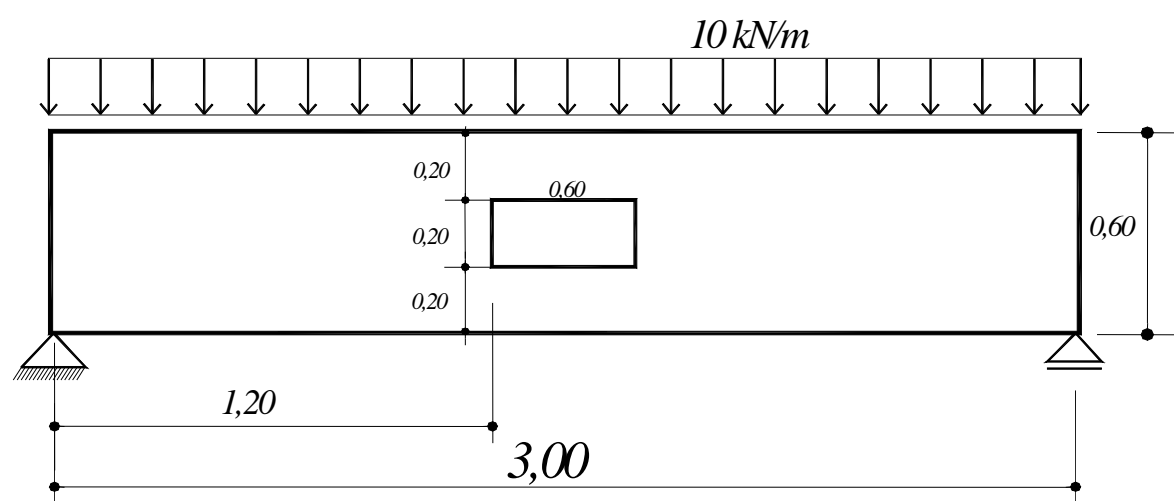

FIGURA 7.7 - Viga simplesmente apoiada com um furo em seu domínio.

A discretização empregada está apresentada na Tabela 7.4: 
TABELA 7.4 - Discretização adotada.

\begin{tabular}{ccc}
\hline Método & Elementos $(\boldsymbol{x}-\boldsymbol{y})$ & G. liberdade \\
\hline & $\begin{array}{c}2 \times(60 \times 8)+2 \times(24 \times 8): 672 \\
\text { (quatro regiões de elementos, } \\
\text { desconsiderando-se o furo) } \\
\text { MEF / QST }\end{array}$ & 6360 \\
MEC & $120 \times 24$ & 1154 \\
\hline
\end{tabular}

Com o intuito de se ter um melhor entendimento do comportamento das distribuições de tensões nesse tipo de viga anisotrópica e do comportamento relativo dos métodos, os resultados dos dois métodos são comparados em cinco seções transversais da viga. São elas: $x=0,40 m ; x=0,60 m ; x=1,0 m ; x=1,16 m$ e a seção referente à face do furo, $x=1,20 \mathrm{~m}$.

Neste sentido, também são feitas comparações com as soluções do MEF/QST para uma viga isotrópica sujeita as mesmas condições geométricas e de carregamento, em duas seções transversais, as seções $x=0,40 \mathrm{~m}$ e $\mathrm{x}=1,20 \mathrm{~m}$. As propriedades elásticas da viga isotrópica estão contidas na Tabela 7.5.

TABELA 7.5 - Constantes elásticas da viga isotrópica

\begin{tabular}{ccc}
\hline Constantes Elásticas & Nomenclatura & Valor (GPa) \\
\hline Módulo de Young na direção global x & $\mathrm{E}_{\mathrm{x}}$ & 19,681 \\
Módulo de Young na direção global y & $\mathrm{E}_{\mathrm{y}}$ & 19,681 \\
Módulo de elasticidade transversal & $\mathrm{G}_{\mathrm{xy}}$ & 9,841 \\
Coeficiente de Poisson & $v_{\mathrm{xy}}$ & 0,0 \\
\hline
\end{tabular}

A seguir, apresentam-se os resultados:

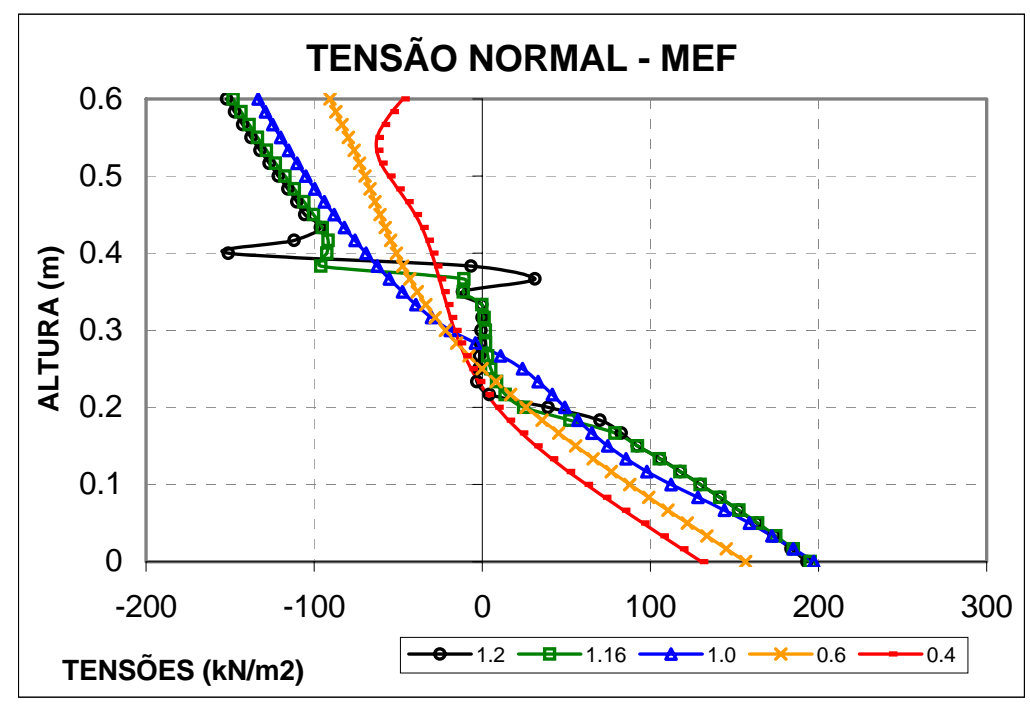

FIGURA 7.8 - Tensões normais para várias seções (MEF). 


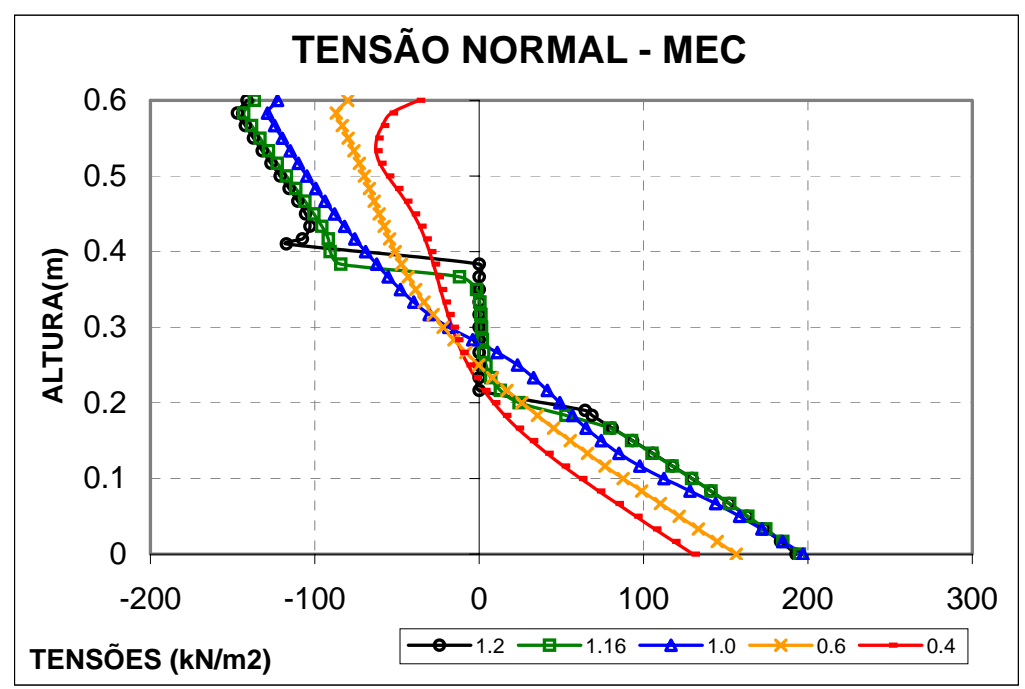

FIGURA 7.9 - Tensões normais para várias seções (MEC).

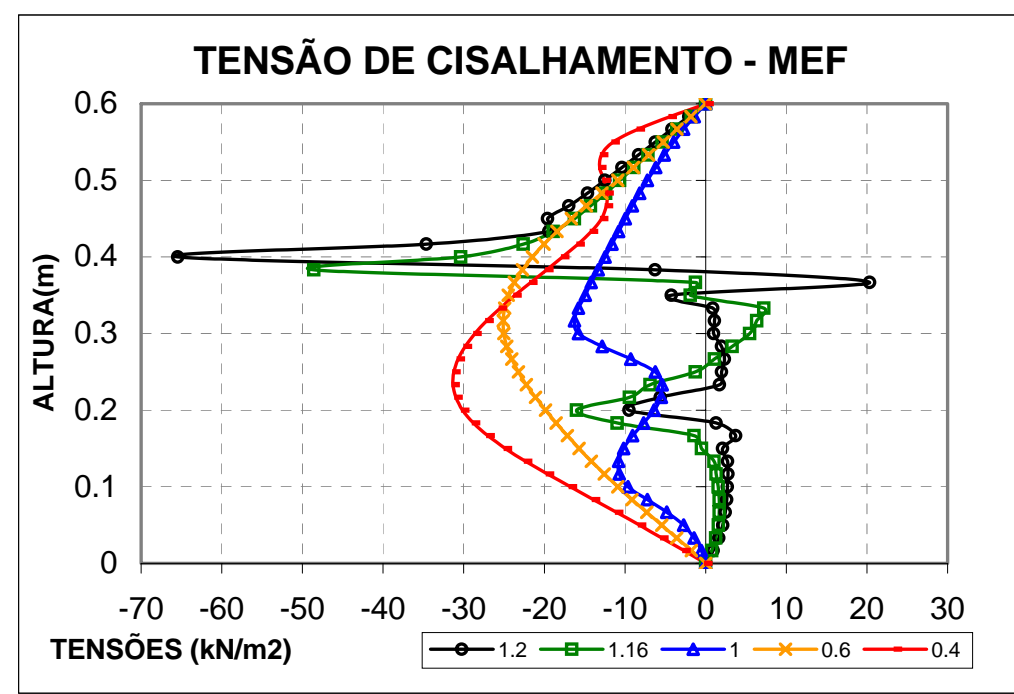

FIGURA 7.10 - Tensões de cisalhamento para várias seções (MEF).

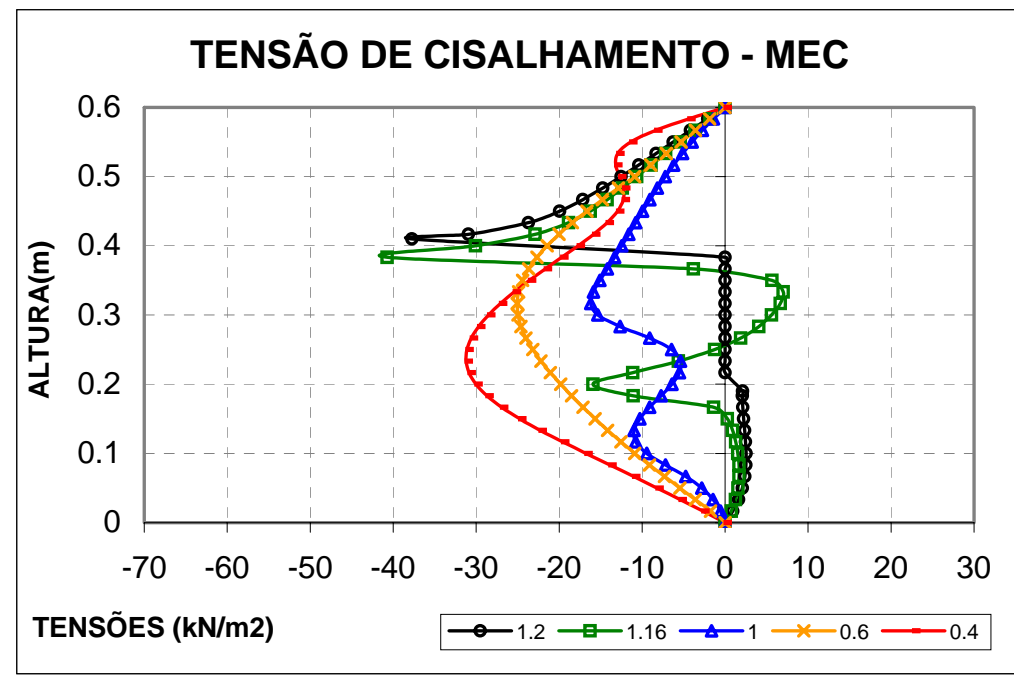

FIGURA 7.11 - Tensões de cisalhamento para várias seções (MEC). 
Nas soluções obtidas, observa-se que ambas tensões são nulas na seção $\mathrm{x}=$ 1,20 m, que corresponde à face esquerda do buraco, ocorrendo uma perturbação nas distribuições no ponto superior inicial do furo $(\mathrm{y}=0,40 \mathrm{~m})$, nos resultados do MEF/QST. A solução obtida com o MEC apresentou um comportamento não oscilatório. Na medida em que as seções analisadas se afastam do buraco, as distribuições de tensões tendem a se estabilizar, assemelhando-se as de uma viga anisotrópica com o domínio contínuo, existindo grande concordância entre as repostas de tensões obtidas com os dois métodos.

A seguir, são comparadas as distribuições de tensões da viga da Figura 7.7 considerando-a primeiramente anisotrópica (Tabela 7.2), e depois, isotrópica possuindo as propriedades elásticas da Tabela 7.5. Assim têm-se:

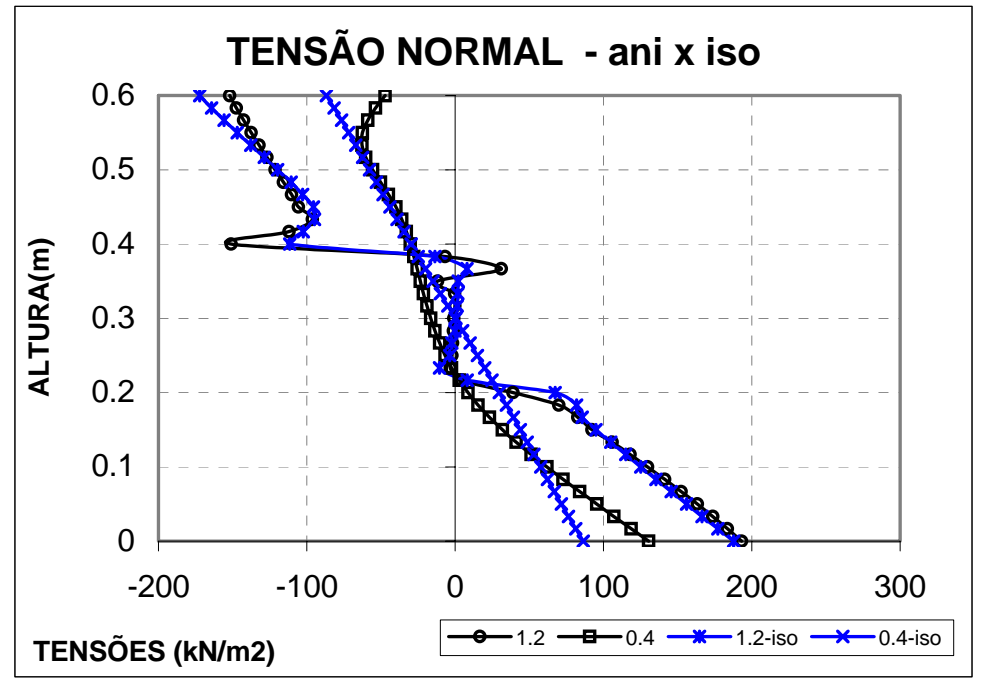

FIGURA 7.12 -Tensões normais de uma viga anisotrópica e de uma isotrópica - (MEC).

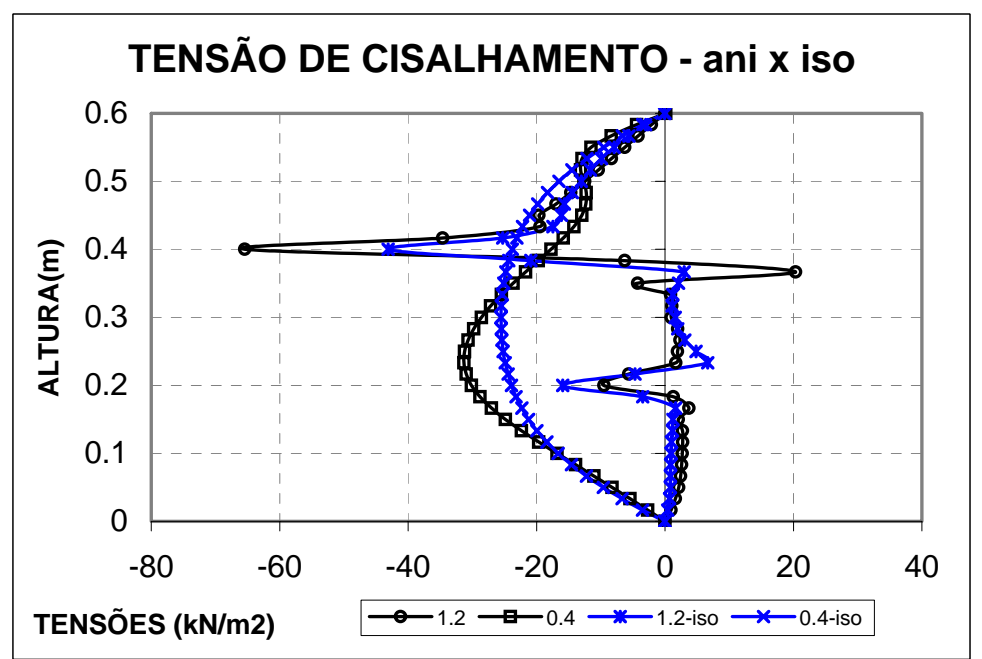

FIGURA 7.13 - Tensões de cisalhamento de uma viga anisotrópica e de uma isotrópica (MEC). 
Nos diagramas anteriores, evidencia-se o comportamento não simétrico das distribuições de tensões em vigas constituídas de materiais anisotrópicos. Uma outra constatação é que na seção correspondente à face do buraco, para a viga isotrópica, surgem também descontinuidades e variações bruscas de tensões semelhantes às das vigas anisotrópicas.

\subsection{EXEMPLO 3 - DETERMINAÇÃO DE FATORES DE INTENSIDADE DE TENSÃO KI}

Mais recentemente, devido a boa performance na representação de campos de tensão em domínios, o MEC tem sido muito utilizado para análise de problemas na Mecânica da Fratura, citando-se, por exemplo, CRUSE (1972), PORTELA et al. (1992) e ALIABADI \& ROOKE (1992). Para domínios anisotrópicos, o primeiro trabalho a abordar a determinação de fatores de intensidade de tensão foi o de SNYDER \& CRUSE (1975). Nesse trabalho pioneiro, os autores utilizaram uma solução fundamental, também baseada na solução fundamental de CRUSE \& SWEDLOW (1971), que já considera no domínio infinito anisotrópico a influência de uma trinca, livre de tensões em suas faces.

No entanto, em todos os trabalhos citados, são utilizadas estratégias específicas para a determinação dos fatores de intensidade de tensão, como por exemplo, método da reciprocidade dual com o elemento quarter-point (ALBUQUERQUE et al., 2002).

O procedimento empregado nesta pesquisa é simples (VANALLI et al., 2003b) e sua principal característica é a discretização da trinca presente no meio anisotrópico, ou quase-isotrópico, utilizando-se apenas elementos de contorno ordinários, ou seja, nenhum elemento de contorno específico para o tratamento de trincas é empregado (MACIEL, 2003), diferenciando-se assim, do trabalho de SNYDER \& CRUSE (1975).

O problema a ser analisado está apresentado na Figura 7.14. Neste exemplo, são determinados fatores de intensidade de tensão para o modo I, porém, a metodologia proposta também pode ser aplicada para a determinação de fatores para o modo II.

Os resultados obtidos com o emprego da técnica proposta são comparados com os obtidos por SNYDER \& CRUSE (1975), por isso, três diferentes chapas ortotrópicas são analisadas e suas dimensões dadas na Tabela 7.6. As mesmas propriedades físicas são adotadas para todas as chapas (Tabela 7.7). 


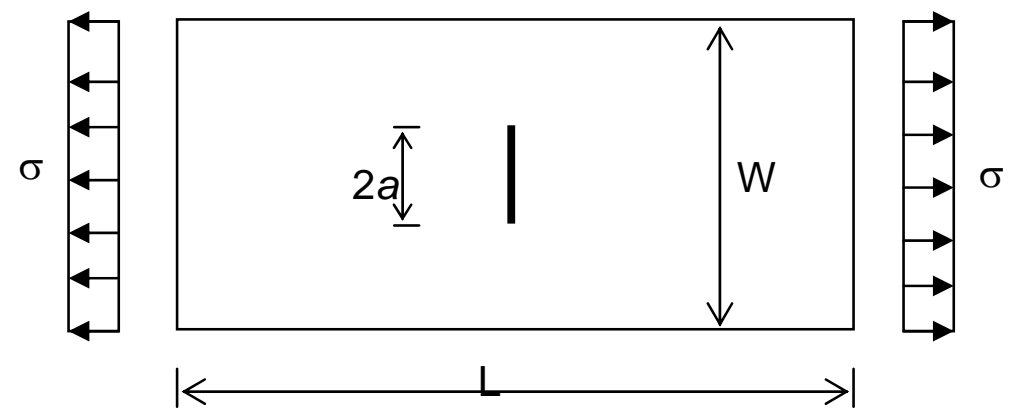

FIGURA 7.14: Geometria geral - Análise estática modo I.

TABELA 7.6 - Propriedades geométricas para três chapas com uma trinca em seu domínio.

\begin{tabular}{|c|c|c|c|c|}
\hline Chapa & $2 \mathrm{a}\left(10^{-3} \mathrm{~m}\right)$ & $\mathrm{W}\left(10^{-3} \mathrm{~m}\right)$ & $L\left(10^{-3} \mathrm{~m}\right)$ & $2 a / W$ \\
\hline 1 & 8 & 20 & 60 & 0,4 \\
\hline 2 & 12 & 20 & 60 & 0,6 \\
\hline 3 & 16 & 20 & 60 & 0,8 \\
\hline
\end{tabular}

TABELA 7.7 - Propriedades elásticas para as chapas ortotrópicas.

\begin{tabular}{ccc}
\hline Propriedades & Nomenclatura & Valores \\
\hline Módulo de Young na direção global x & $\mathrm{E}_{1}$ & $147,57(\mathrm{GPa})$ \\
Módulo de Young na direção global y & $\mathrm{E}_{2}$ & $11,95(\mathrm{GPa})$ \\
Módulo de elasticidade transversal & $\mathrm{G}_{12}$ & $9,84(\mathrm{GPa})$ \\
Coeficiente de Poisson & $v_{12}$ & 0,21 \\
Abertura da trinca & $\mathrm{h}$ & $2 \mathrm{a} \mathrm{x} 10^{-6}(\mathrm{~m})$ \\
Tensão uniforme aplicada & $\sigma$ & $1,0(\mathrm{kPa})$ \\
\hline
\end{tabular}

A Equação (7.2) é utilizada juntamente com a formulação de elementos de contorno para se realizar as comparações dos valores dos fatores de intensidade de tensão. As chapas laminadas são compostas de 4 lâminas organizadas segundo as direções de reforço $\left(90 / \pm 45^{\circ}\right)$ s, como feito por SNYDER \& CRUSE (1975).

$$
Y=\frac{K_{I}}{\sigma \cdot \sqrt{a}}
$$

Assim, de posse das informações apresentadas, pode-se então comparar as soluções obtidas com os resultados de SNYDER \& CRUSE (1975), Tabela 7.8: 
TABELA 7.8 - Comparações dos valores de $\boldsymbol{Y}$.

\begin{tabular}{ccccc}
\hline chapa & $\mathbf{K}_{\mathbf{1} \text { anisotropico }}$ & $\mathbf{Y}_{\text {anisotropico }}$ & $\mathbf{Y}_{\text {Snyder\&cruse }}$ & Variação (\%) \\
\hline 1 & 3,821 & 1,911 & 1,972 & 3,09 \\
2 & 5,497 & 2,244 & 2,323 & 3,40 \\
3 & 8,700 & 3,076 & 3,231 & 4,80 \\
\hline
\end{tabular}

Dos resultados apresentados pode-se concluir que mesmo sem a utilização de elementos de contorno especiais para análise de trincas, ou procedimentos de integração, a técnica proposta é de fácil implementação e fornece bons resultados. Na Tabela 7.9 é mostrada a discretização adotada em cada chapa.

TABELA 7.9 - Discretização e pontos de Gauss adotados nos exemplos.

\begin{tabular}{cccc}
\hline Chapa & $\begin{array}{c}\text { EC no contorno } \\
\text { do corpo }\end{array}$ & $\begin{array}{c}\text { EC na superfície } \\
\text { da trinca }\end{array}$ & $\begin{array}{c}\text { Pontos de } \\
\text { Gauss }\end{array}$ \\
\hline 1 & 80 & 60 & 24 \\
2 & 80 & 64 & 24 \\
3 & 80 & 64 & 32 \\
\hline
\end{tabular}

É conveniente ainda comentar que a estratégia empregada para a extração dos fatores de intensidade de tensão é a mesma utilizada por MACIEL (2003), seguindo procedimentos similares aos apresentados por PARIS \& CANÃS (1997). O esquema de extração consiste na realização de uma média entre os valores extraídos para os fatores de intensidades de tensão em três pontos afastados a certas distâncias da ponta da trinca. Para o caso anisotrópico aqui apresentado, os pontos de extração adotados foram $a / 30$, $a / 40$ e $a / 50$, conforme pode ser verificado na Figura 7.15:

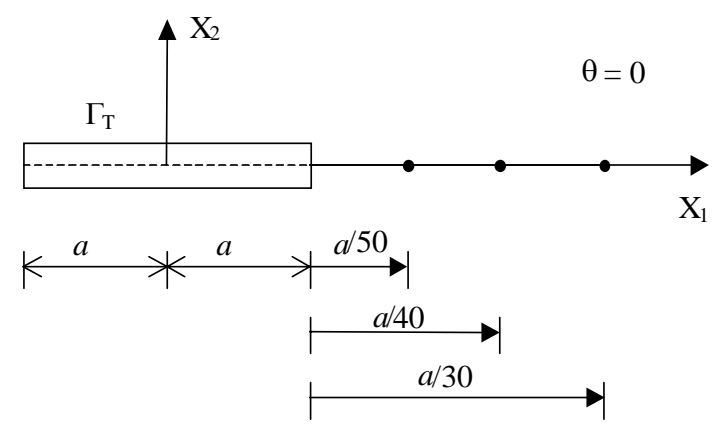

FIGURA 7.15 - Pontos de extração para a análise anisotrópica.

Dessa maneira, conclui-se este capítulo e no seguinte apresenta-se alguns exemplos onde as formulações viscoelásticas do MEF e do MEC foram empregadas. 


\section{APLICAÇõES - ANÁLISE VISCOELÁSTICA}

Neste capítulo são apresentados dois exemplos onde a formulação viscoelástica para meios anisotrópicos é aplicada. No primeiro, uma chapa tracionada quaseisotrópica é analisada e as soluções obtidas, por meio do MEF/QST e do MEC, são comparadas entre si e também com as soluções, em termos de tensões e de deslocamentos, apresentadas por MESQUITA \& CODA (2002), os quais estudaram a mesma chapa, porém, considerando-a isotrópica tridimensional. No segundo exemplo, o mesmo problema é analisado, porém a chapa considerada possui anisotropia geral e as soluções obtidas com o MEF/QST e com MEC são simplesmente confrontadas no sentido de sua verificação.

\subsection{EXEMPLO 1 - CHAPA QUASE-ISOTRÓPICA}

A chapa a ser analisada está apresentada na Figura 8.1:

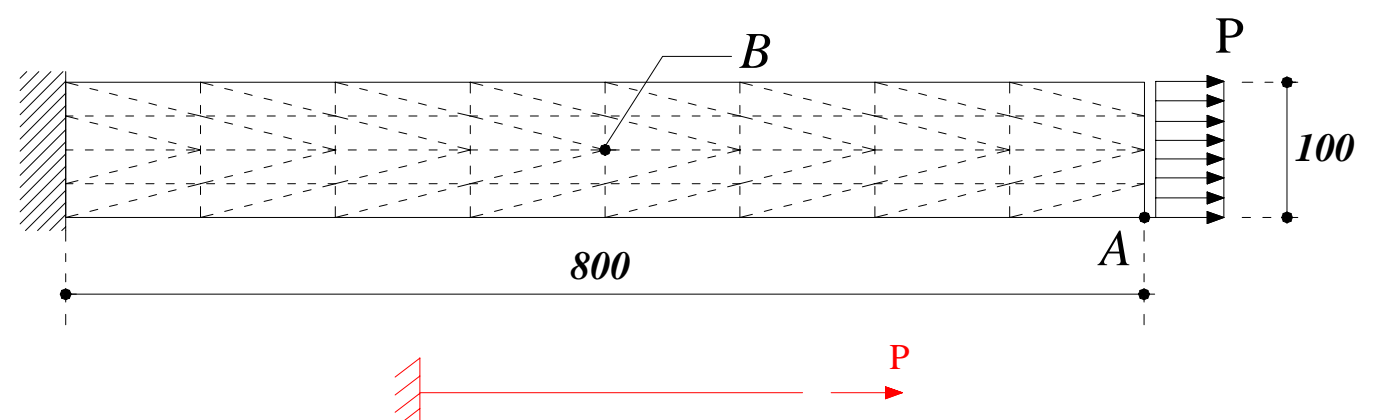

FIGURA 8.1 - Chapa com dimensões de 800mm x 100mm. 
e suas propriedades elásticas, bem como os parâmetros para a análise viscosa estão apresentados na Tabela 8.1:

TABELA 8.1 - Parâmetros elásticos e de viscosidade da chapa quase-isotrópica

\begin{tabular}{ccc}
\hline Parâmetros & Nomenclatura & Valor \\
\hline Módulo de Young na direção global x & $\mathrm{E}_{\mathrm{x}}$ & $1,0 \mathrm{kN} / \mathrm{mm}^{2}$ \\
Módulo de Young na direção global y & $\mathrm{E}_{\mathrm{y}}$ & $0,9 \mathrm{kN} / \mathrm{mm}^{2}$ \\
Módulo de elasticidade transversal & $\mathrm{G}_{\mathrm{xy}}$ & $0,5 \mathrm{kN} / \mathrm{mm}^{2}$ \\
Coeficiente de Poisson & $v_{\mathrm{xy}}$ & 0,0 \\
Tamanho do incremento de tempo & $\Delta \mathrm{t}$ & $1 \mathrm{dia}$ \\
Número de incrementos de tempo & - & 450 \\
Parâmetro de viscosidade & $\gamma$ & $45,4545 \mathrm{dias}$ \\
Carregamento aplicado & $\mathrm{P}_{\mathrm{x}}$ & $0,005 \mathrm{kN}$ \\
\hline
\end{tabular}

Como feito por MESQUITA \& CODA (2002), é utilizada na análise pelo MEF a discretização apresentada na Figura 8.1 (16 x 8 elementos). Com o MEC, são empregados 8 elementos de contorno na direção $x$ e 4 na direção $y$. Desta maneira, com as informações apresentadas, pode-se então construir alguns gráficos comparativos. As soluções são apresentadas para os pontos $\boldsymbol{A}$ e $\boldsymbol{B}$ da Figura 8.1. Assim, para os deslocamentos no ponto $\boldsymbol{A}$ do contorno tem-se:

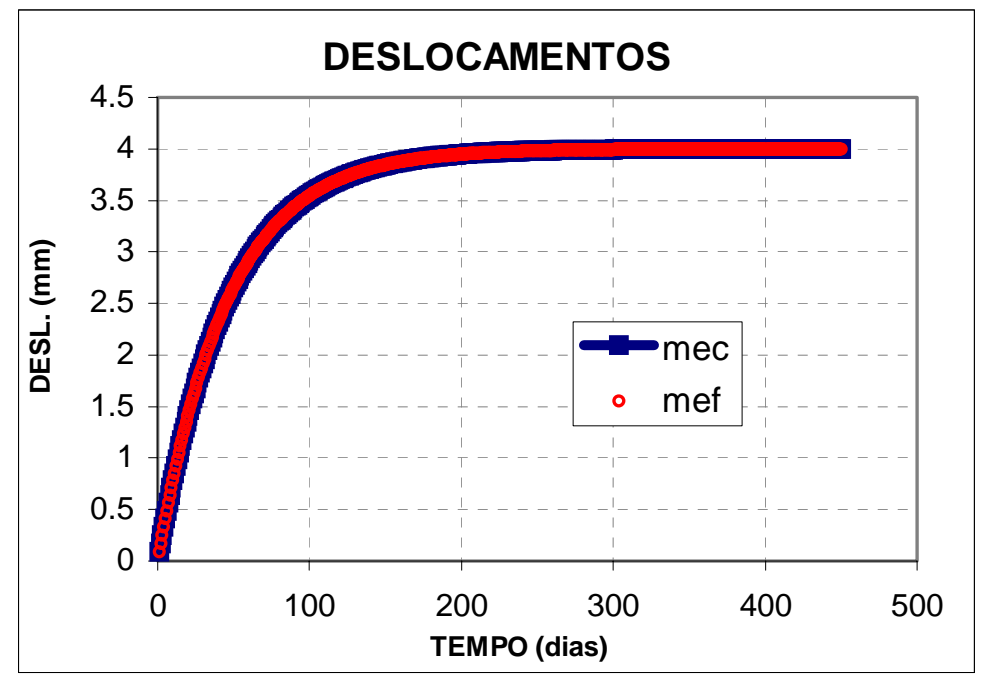

FIGURA 8.2 - Deslocamentos para o ponto A do contorno - MEC e MEF. 
e para o ponto $\boldsymbol{B}$ pertencente ao domínio:

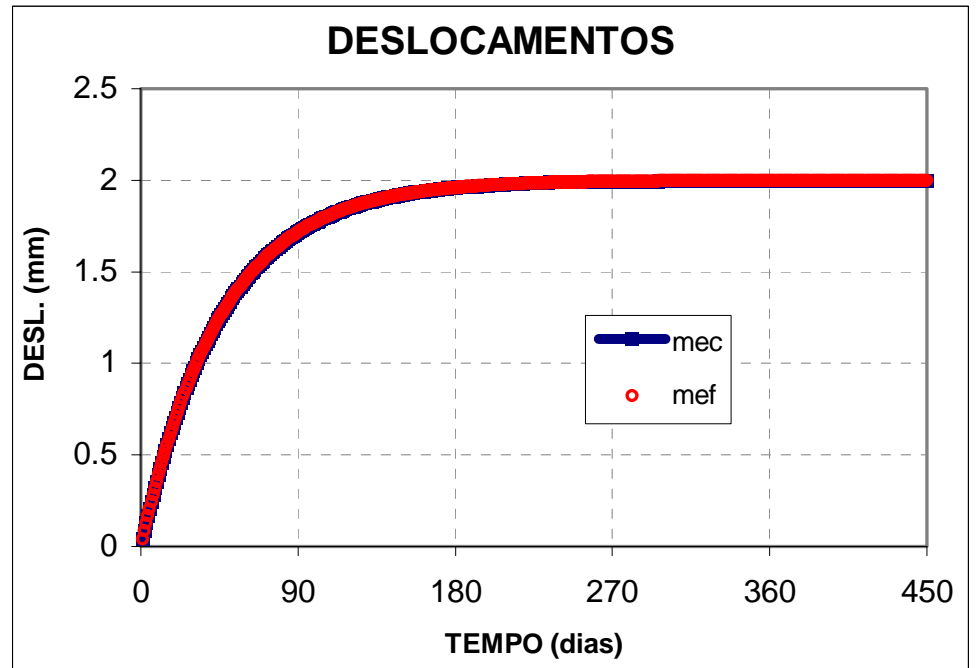

FIGURA 8.3 - Deslocamentos para o ponto $B$ do domínio - MEC e MEF.

Para as tensões normais (elástica, viscosa e total) no ponto $\boldsymbol{A}$ :

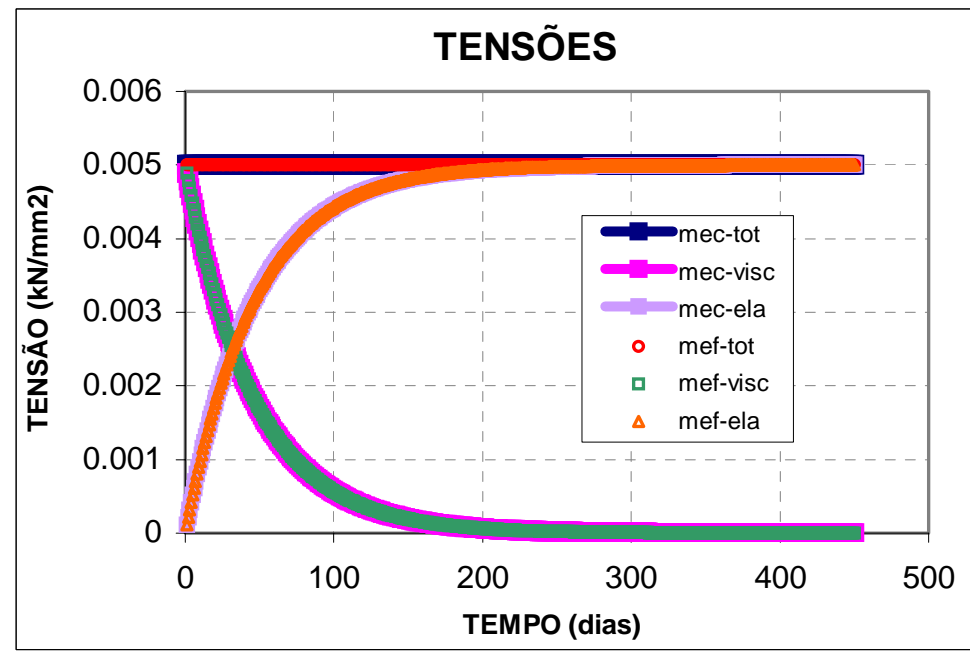

FIGURA 8.4 - Tensões elásticas, viscosa e total para o ponto A - MEC e MEF. 
e para o ponto $\boldsymbol{B}$ :

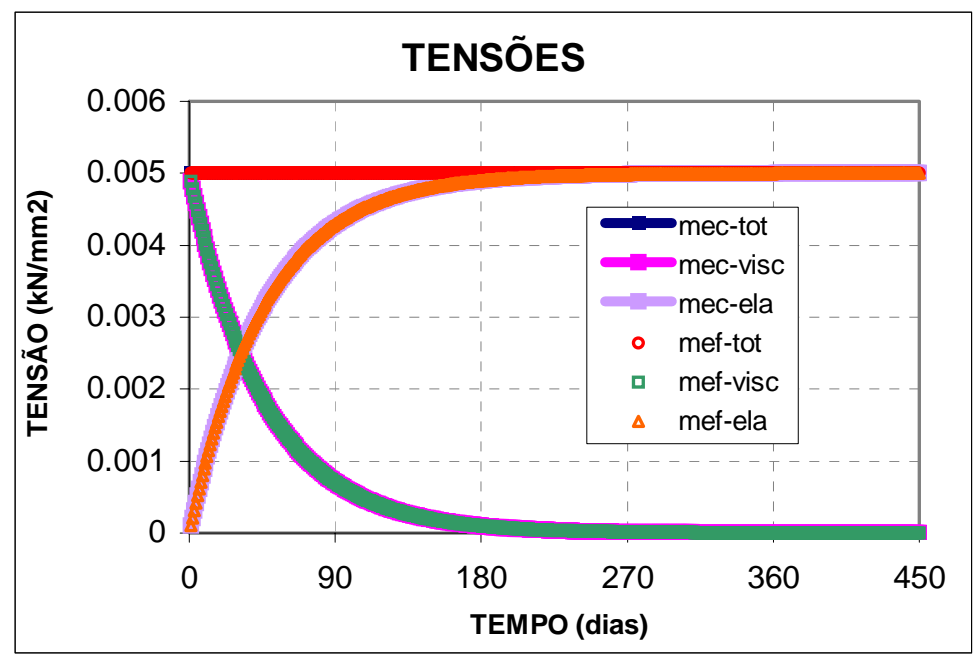

FIGURA 8.5 - Tensões elásticas, viscosa e total para o ponto $B$ - MEC e MEF.

Salienta-se que os resultados apresentados são idênticos aos obtidos por MESQUITA \& CODA (2002).

\subsection{EXEMPLO 2 - CHAPA COM ANISOTROPIA GERAL}

A chapa analisada é a mesma apresentada na Figura 8.1, porém, com as propriedades elásticas apresentadas na Tabela 8.2:

TABELA 8.2 - Parâmetros elásticos e de viscosidade da chapa anisotrópica

\begin{tabular}{|c|c|c|}
\hline Constantes elásticas & Nomenclatura & Valor \\
\hline Módulo de Young na direção global x & $\overline{E_{x}}$ & $0,73 \mathrm{kN} / \mathrm{mm}^{2}$ \\
\hline Módulo de Young na direção global y & $\mathrm{E}_{\mathrm{y}}$ & $1,14 \mathrm{kN} / \mathrm{mm}^{2}$ \\
\hline Módulo de elasticidade transversal & $\mathrm{G}_{\mathrm{xy}}$ & $0,28 \mathrm{kN} / \mathrm{mm}^{2}$ \\
\hline Coeficiente de Poisson & $v_{\mathrm{xy}}$ & 0,14 \\
\hline $\begin{array}{l}\text { Coeficiente de influência mútua } \\
\left.\text { ( } 1^{\text {a }} \text {.espécie }\right)\end{array}$ & $\eta_{x y, x}$ & 0,0 \\
\hline $\begin{array}{l}\text { Coeficiente de influência mútua } \\
\left.\text { ( } 2^{\mathrm{a}} \text {.espécie }\right)\end{array}$ & $\eta_{x y, y}$ & $-0,63$ \\
\hline
\end{tabular}


As soluções também são apresentadas para os pontos $A$ e $B$ da chapa:

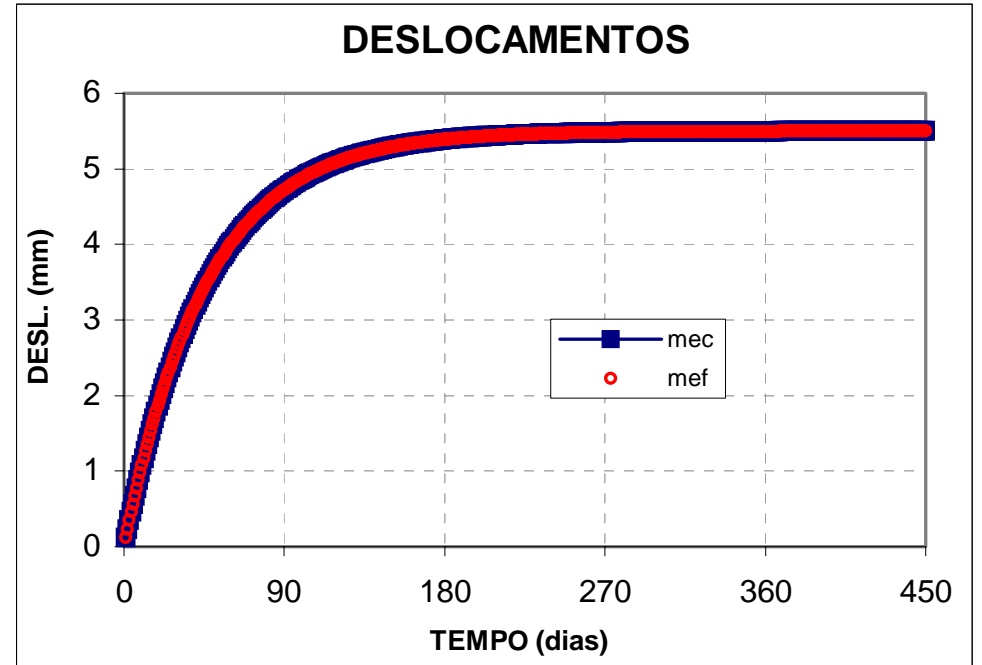

FIGURA 8.6 - Deslocamentos para o ponto A do contorno - MEC e MEF.

e para o ponto $\boldsymbol{B}$ pertencente ao domínio:

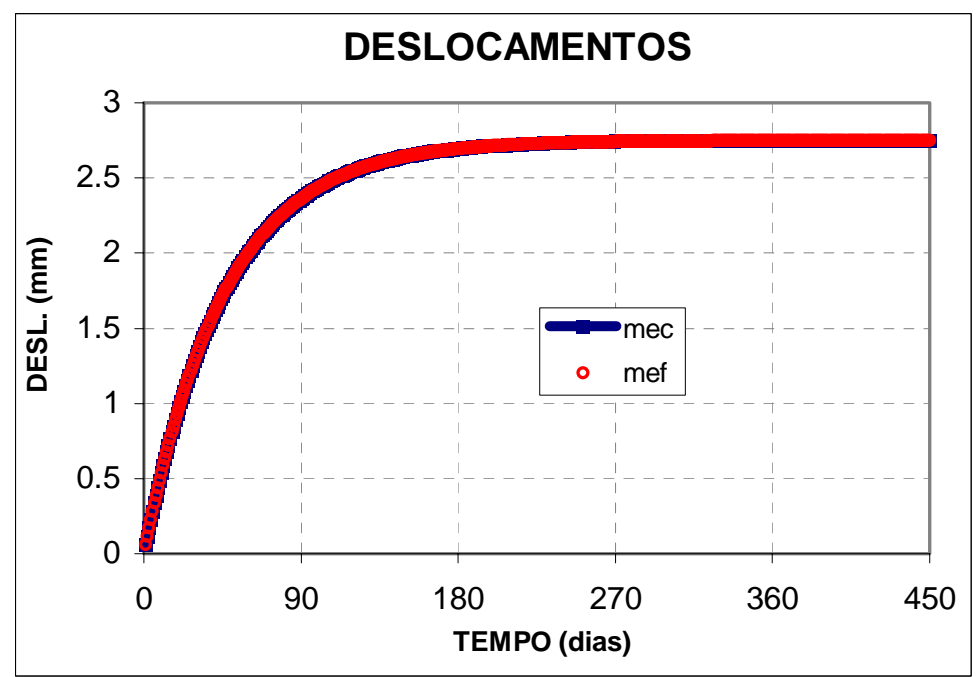

FIGURA 8.7 - Deslocamentos para o ponto B do domínio - MEC e MEF. 
Para as tensões normais (elástica, viscosa e total) no ponto $\boldsymbol{A}$ :

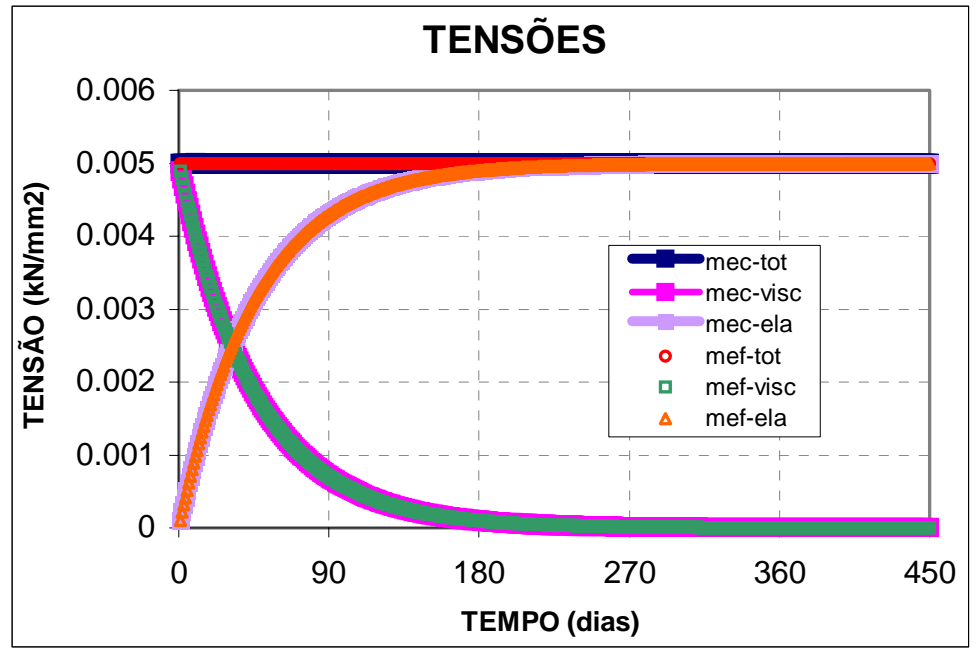

FIGURA 8.8 - Tensões elásticas, viscosa e total para o ponto A - MEC e MEF.

e para o ponto $\boldsymbol{B}$ :

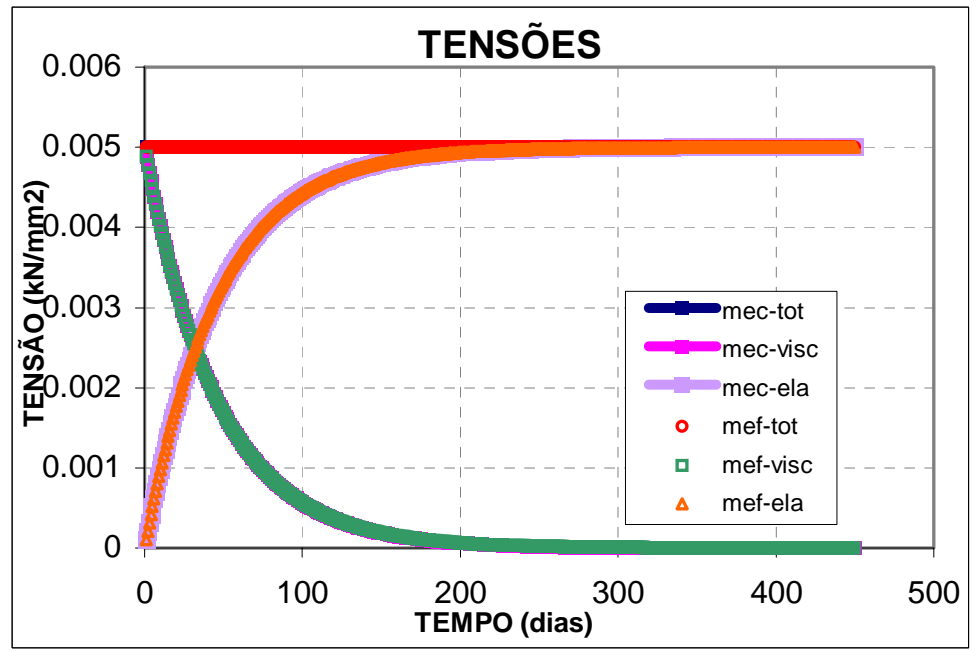

FIGURA 8.9 - Tensões elásticas, viscosa e total para o ponto $B$ - MEC e MEF.

É importante comentar que a simulação do comportamento mecânico dos materiais anisotrópicos ao longo do tempo, utilizando-se a metodologia diferencial (MESQUITA \& CODA, 2002), é um assunto ainda aberto para pesquisas e, por isso, o estudo realizado certamente poderá servir de parâmetro para outros trabalhos que envolvam viscoelasticidade em meios anisotrópicos. 


\section{APLICAÇõES - ANÁLISE VISCOPLÁSTICA}

Neste capítulo são apresentados três exemplos onde a formulação viscoplástica para meios anisotrópicos é aplicada. Salienta-se que pelo fato da formulação elastoplástica já estar, de forma implícita, considerada na formulação viscoplástica, não são apresentados exemplos que envolvam somente análises elastoplásticas. Como já comentado, a plasticidade considerada é a não associativa e nos exemplos aqui analisados adota-se a direção do fluxo plástico equivalente à direção das deformações elásticas, ou seja, direção determinada pelo tensor constitutivo anisotrópico plástico $\left(C_{i j k l}^{p}\right)$.

No primeiro exemplo apresentado uma chapa tracionada quase isotrópica é analisada e as soluções obtidas, por meio do MEF/QST e do MEC, são comparadas entre si e também com as soluções, em termos de tensões e de deslocamentos, obtidas por MESQUITA (2002) o qual estudou a mesma chapa, porém, considerando-a isotrópica 3D. Nesse exemplo são feitas aplicações com encruamentos positivos, negativos e também, com plasticidade perfeita.

No segundo exemplo a chapa analisada no capítulo 8, com viscoelasticidade quase isotrópica, é novamente estudada considerando-se uma tensão de escoamento acima da qual o material apresentará um encruamento positivo. Alteram-se também algumas propriedades elásticas do material utilizado. As soluções obtidas com o MEF/QST e com MEC são simplesmente confrontadas no sentido de sua verificação. No mesmo exemplo, com a utilização de um coeficiente de Poisson na fase plástica igual a 0,5 foi feita também uma análise sobre o comportamento incompressível do material. 
No terceiro exemplo se estuda uma chapa tracionada com anisotropia geral. Considera-se a chapa constituída de um material reforçado por fibras, cujas propriedades elásticas, em relação ao sistema local das fibras, são ortotrópicas. Na análise, feita em relação ao sistema global da chapa, o material pode então ser considerado anisotrópico geral. Nesse exemplo, com a utilização do critério de TSAI \& WU (1971), diferentes resistências ao escoamento, nas duas direções principais, puderam ser consideradas aumentando-se assim, a complexidade da análise.

\subsection{EXEMPLO 1 - CHAPA QUASE-ISOTRÓPICA}

A chapa a ser analisada está apresentada na Figura 9.1:

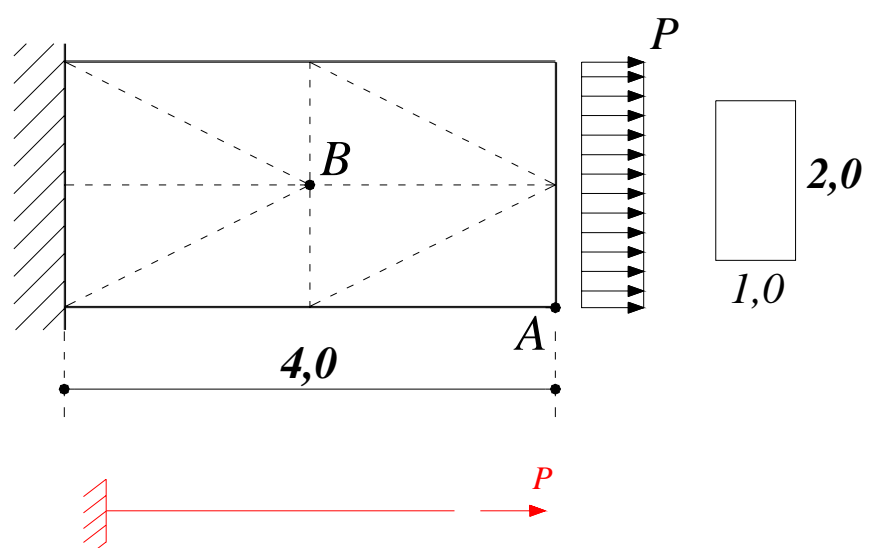

FIGURA 9.1 - Chapa com dimensões de 4m x 2m.

e suas propriedades elásticas, bem como, os parâmetros para a análise viscoplástica estão apresentados na Tabela 9.1: 
TABELA 9.1 - Parâmetros elásticos e de viscoplasticidade da chapa quase-isotrópica

\begin{tabular}{ccc}
\hline Parâmetros & Nomenclatura & Valor \\
\hline Módulo de Young na direção global x & $\mathrm{E}_{\mathrm{x}}$ & $100000 \mathrm{~Pa}$ \\
Módulo de Young na direção global y & $\mathrm{E}_{\mathrm{y}}$ & $90000 \mathrm{~Pa}$ \\
Módulo de elasticidade transversal & $\mathrm{G}_{\mathrm{xy}}$ & $50000 \mathrm{~Pa}$ \\
Coeficiente de Poisson & $v_{\mathrm{xy}}$ & 0,25 \\
Tamanho do incremento de tempo & $\Delta \mathrm{t}$ & $0,01 \mathrm{dia}$ \\
Número de incrementos de tempo & - & 5000 \\
Parâmetro de viscosidade & $\gamma$ & 4,5 dias \\
Carregamento aplicado & $\mathrm{P}_{\mathrm{x}}$ & $1,0 \mathrm{~Pa}$ \\
Tensão de escoamento & $\sigma_{0}$ & $0,45 \mathrm{~Pa}$ \\
Módulo de elasticidade tangente \\
$\begin{array}{c}\text { positivo } \\
\text { Módulo de elasticidade tangente } \\
\text { negativo (softening) }\end{array}$ & $\mathrm{Et}^{+}$ & $+0,10 \mathrm{E}_{\mathrm{x}}$ \\
\hline
\end{tabular}

Deve-se comentar que para este exemplo $C_{i j k l}^{p}=C_{i j k l}^{e}$, ou seja, o fluxo plástico é igual ao fluxo elástico. É utilizada, na análise pelo MEF, a discretização apresentada na Figura 9.1 (4 x 4 elementos). Com o MEC, são empregados 2 elementos de contorno na direção $x$ e 2 na direção $y$. As soluções são apresentadas para os pontos $A$ e $B$ da Figura 9.1, considerando-se os dois tipos de encruamento e a plasticidade perfeita. Para os deslocamentos no ponto $A$, tem-se:

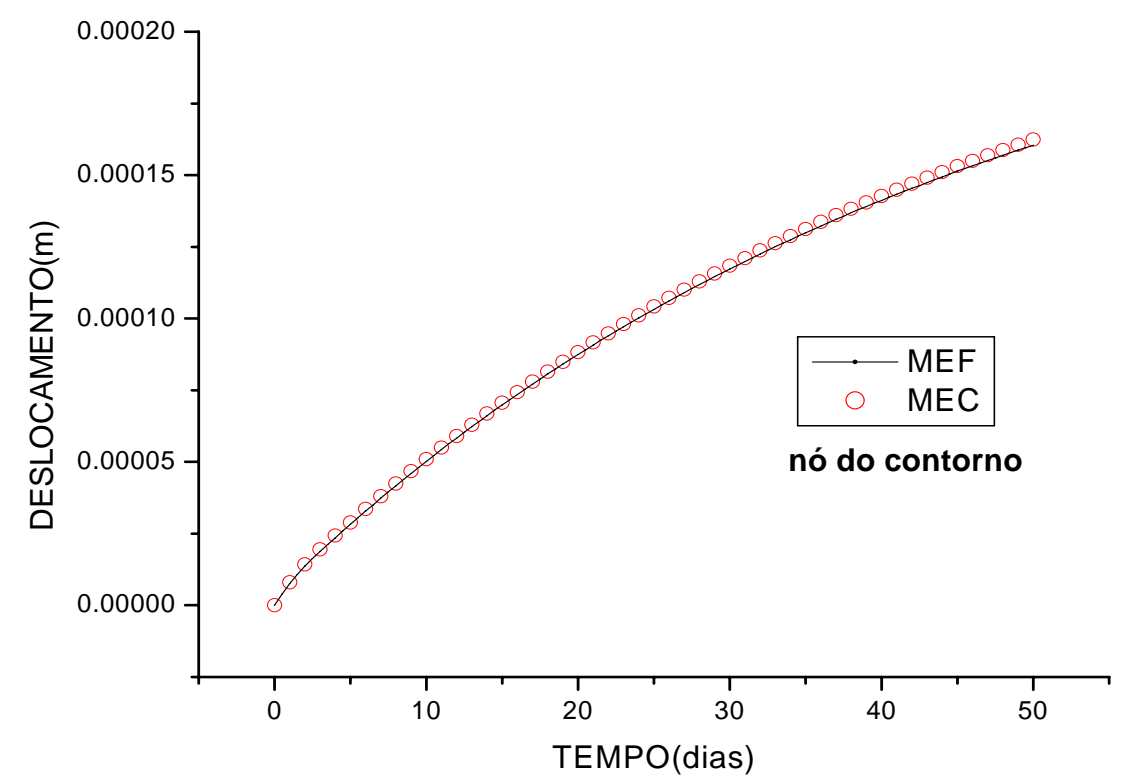

FIGURA 9.2 - Deslocamentos para o ponto A do contorno - encruamento positivo. 


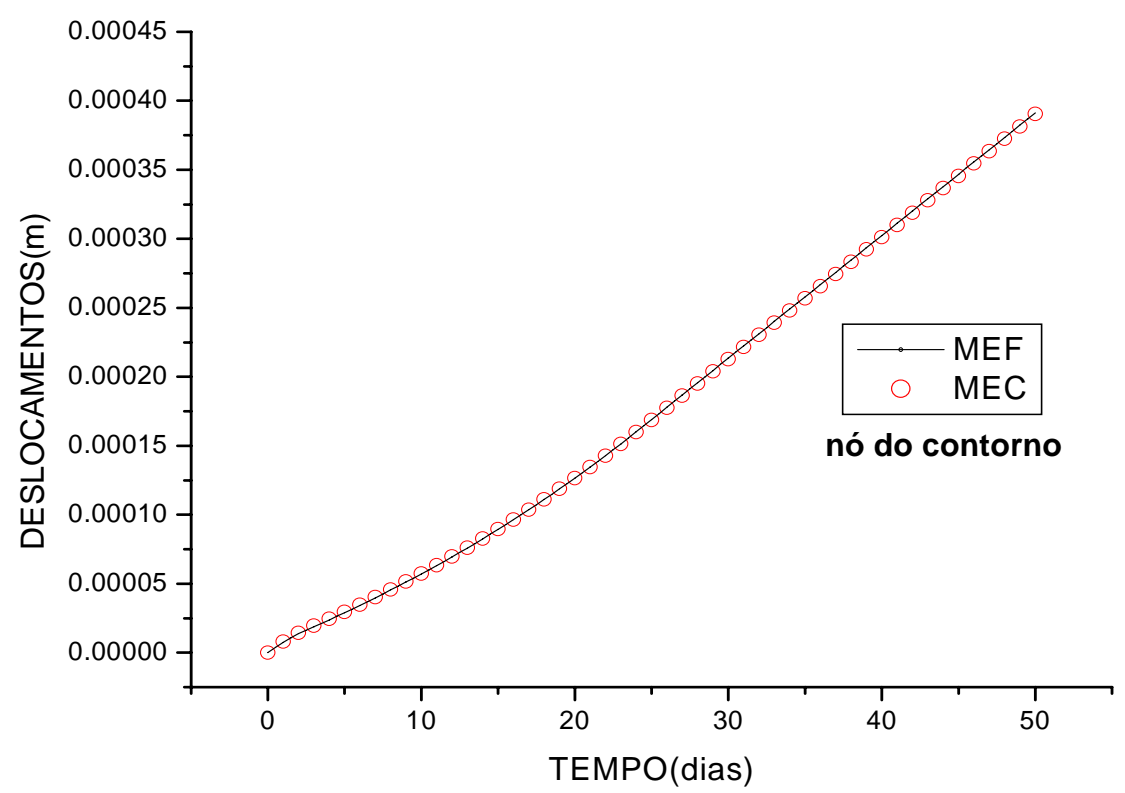

FIGURA 9.3 - Deslocamentos para o ponto A do contorno - encruamento negativo.

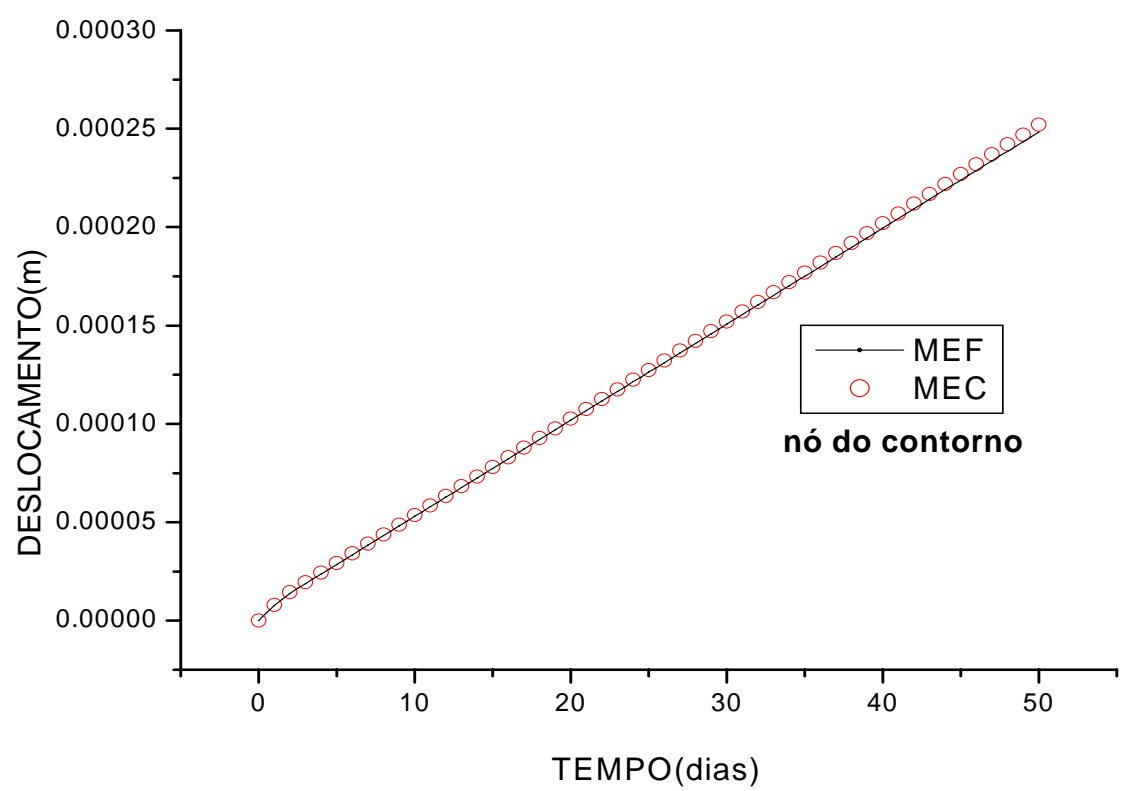

FIGURA 9.4 - Deslocamentos para o ponto A do contorno - plasticidade perfeita. 
e para o ponto $\boldsymbol{B}$ pertencente ao domínio:

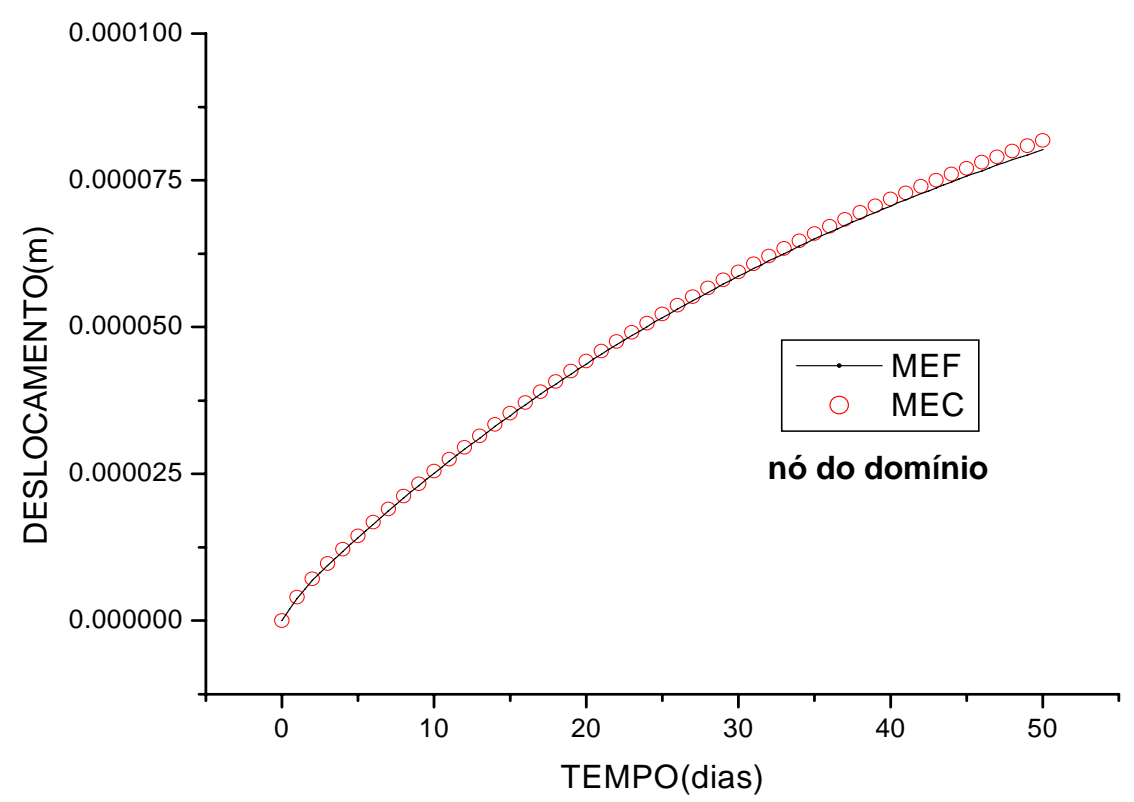

FIGURA 9.5 - Deslocamentos para o ponto B do domínio - encruamento positivo.

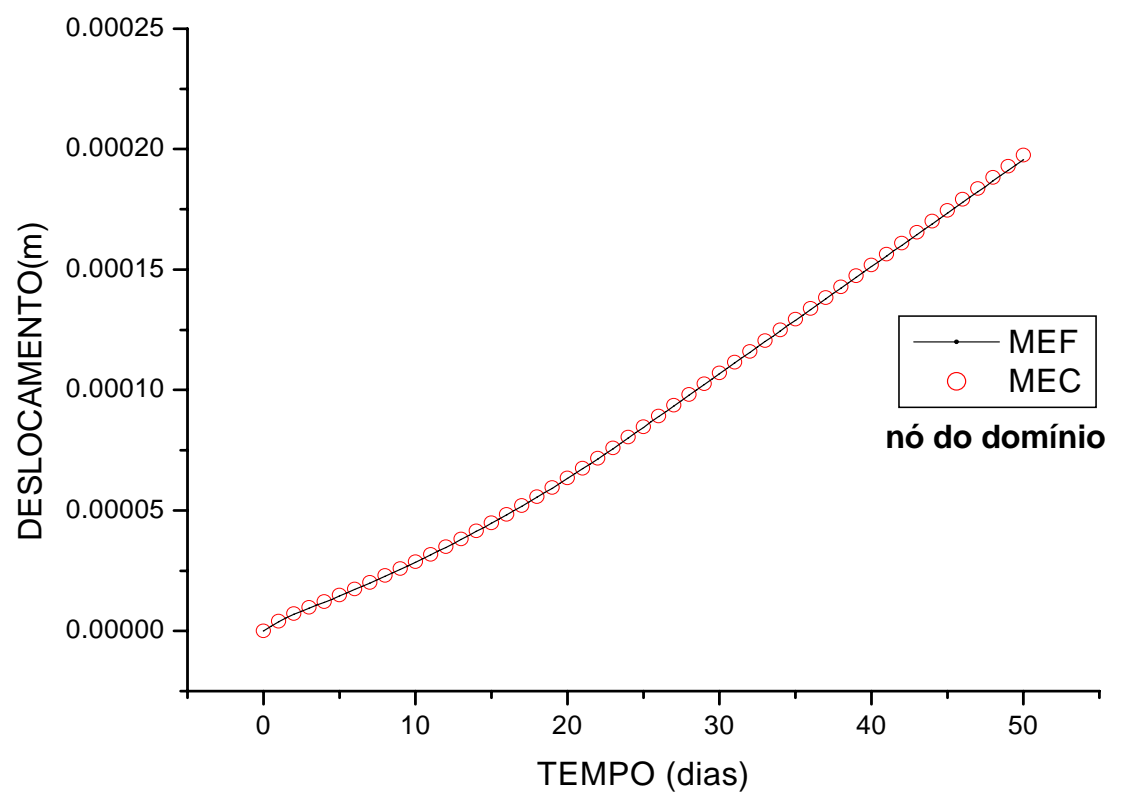

FIGURA 9.6 - Deslocamentos para o ponto B do domínio - encruamento negativo. 


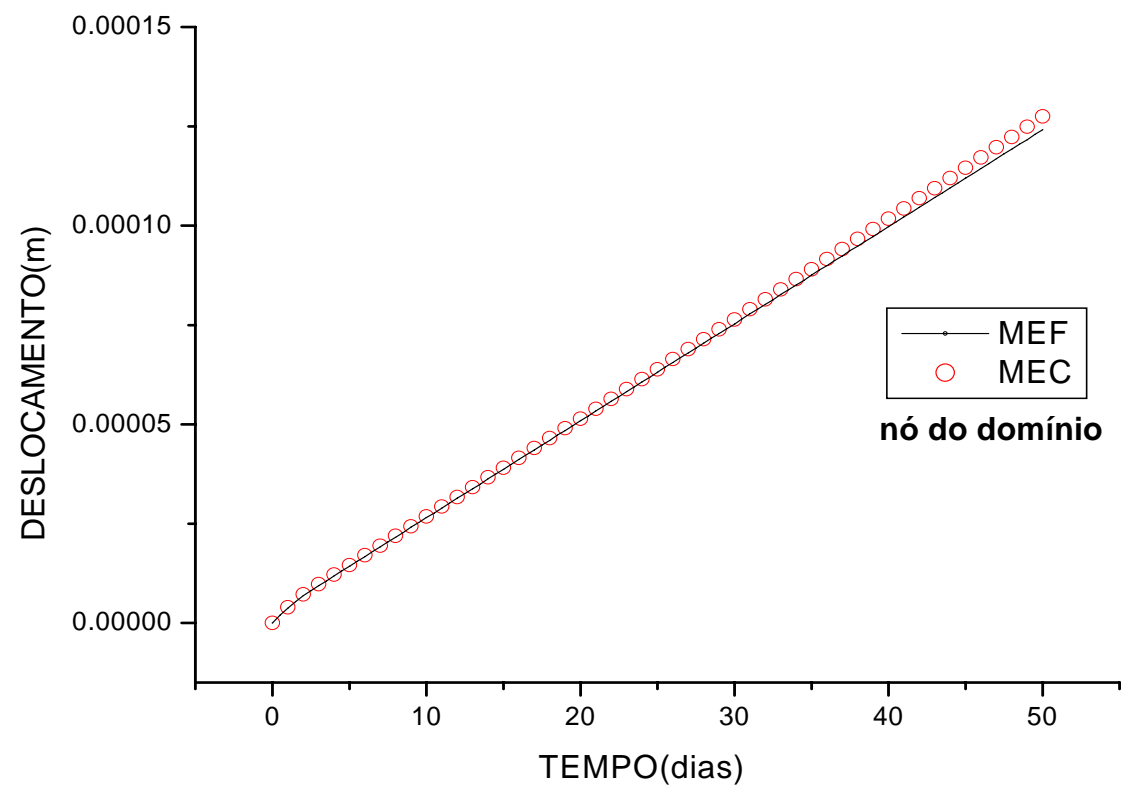

FIGURA 9.7 - Deslocamentos para o ponto B do domínio - plasticidade perfeita.

Para as tensões normais (elastoplástica, viscosa e total) no ponto $\boldsymbol{A}$ :

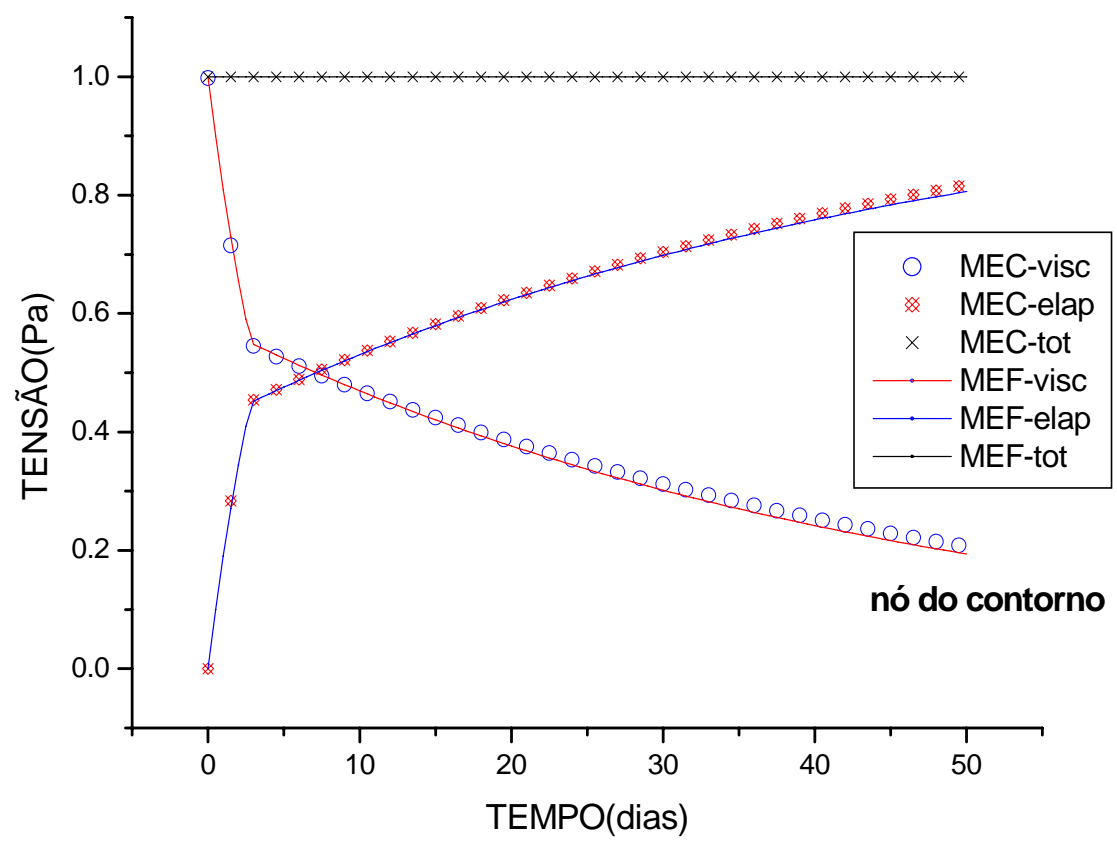

FIGURA 9.8 - Tensões elastoplástica, viscosa e total para o ponto $A$ - encruamento positivo. 


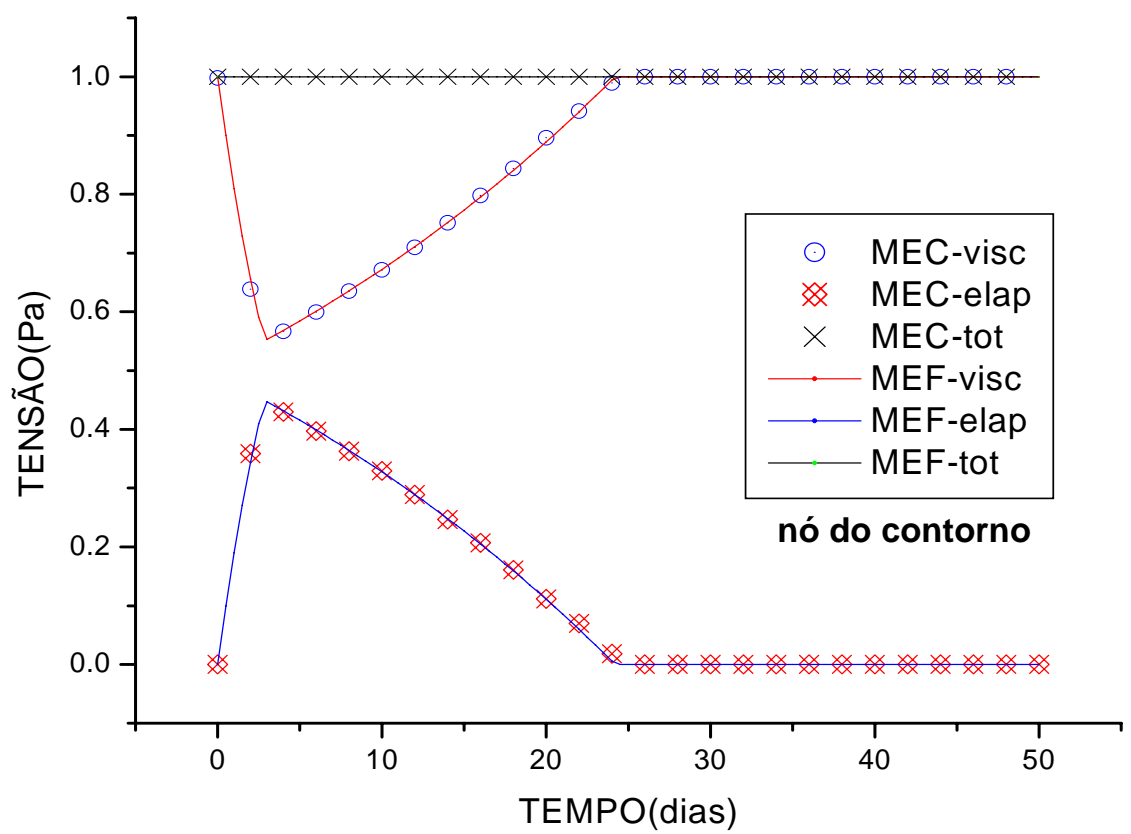

FIGURA 9.9 - Tensões elastoplástica, viscosa e total para o ponto $A$ - encruamento negativo.

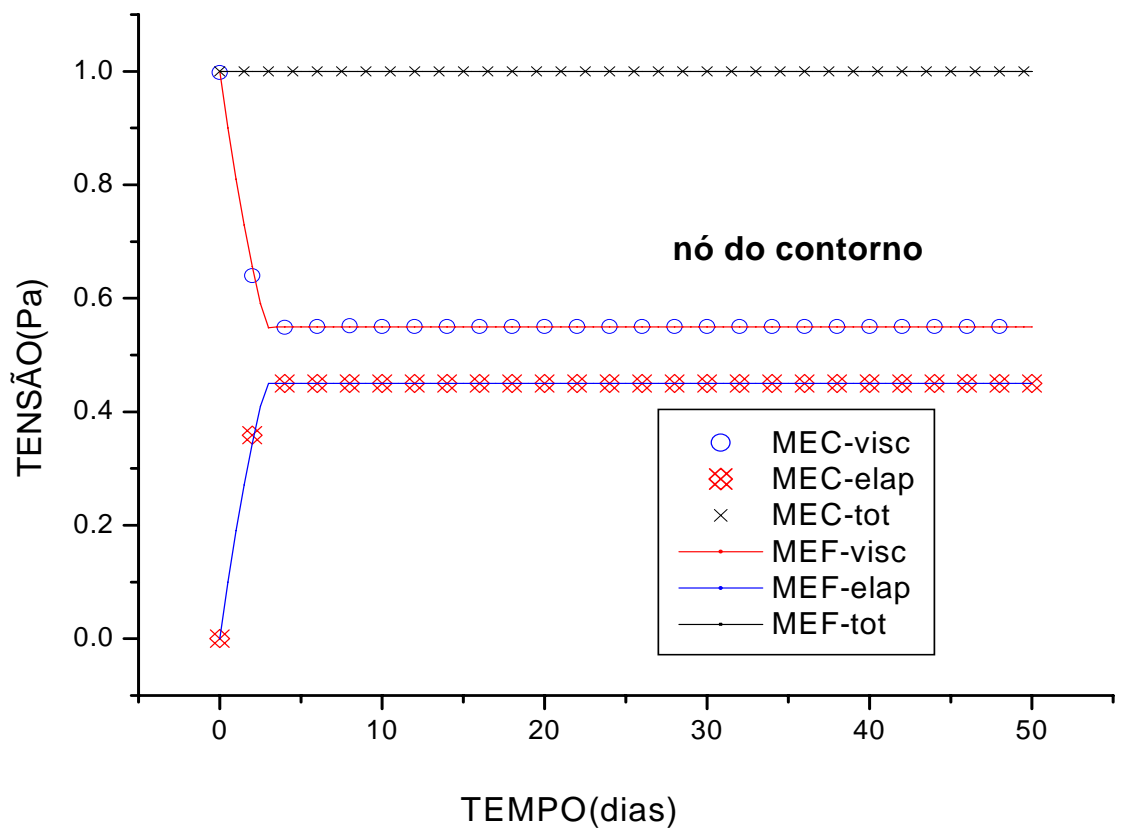

FIGURA 9.10 - Tensões elastoplástica, viscosa e total para o ponto $A$ - plasticidade perfeita. 
e para o ponto $\boldsymbol{B}$ :

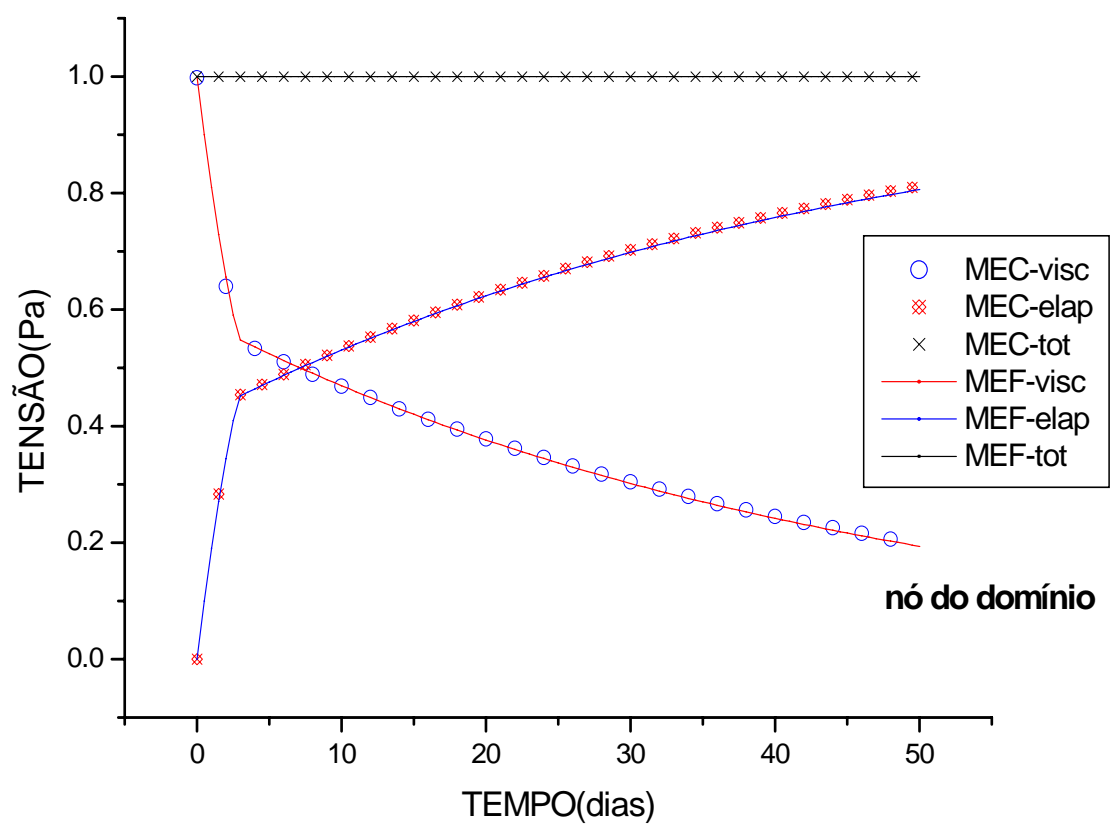

FIGURA 9.11 - Tensões elastoplástica, viscosa e total para o ponto $B$ - encruamento positivo.

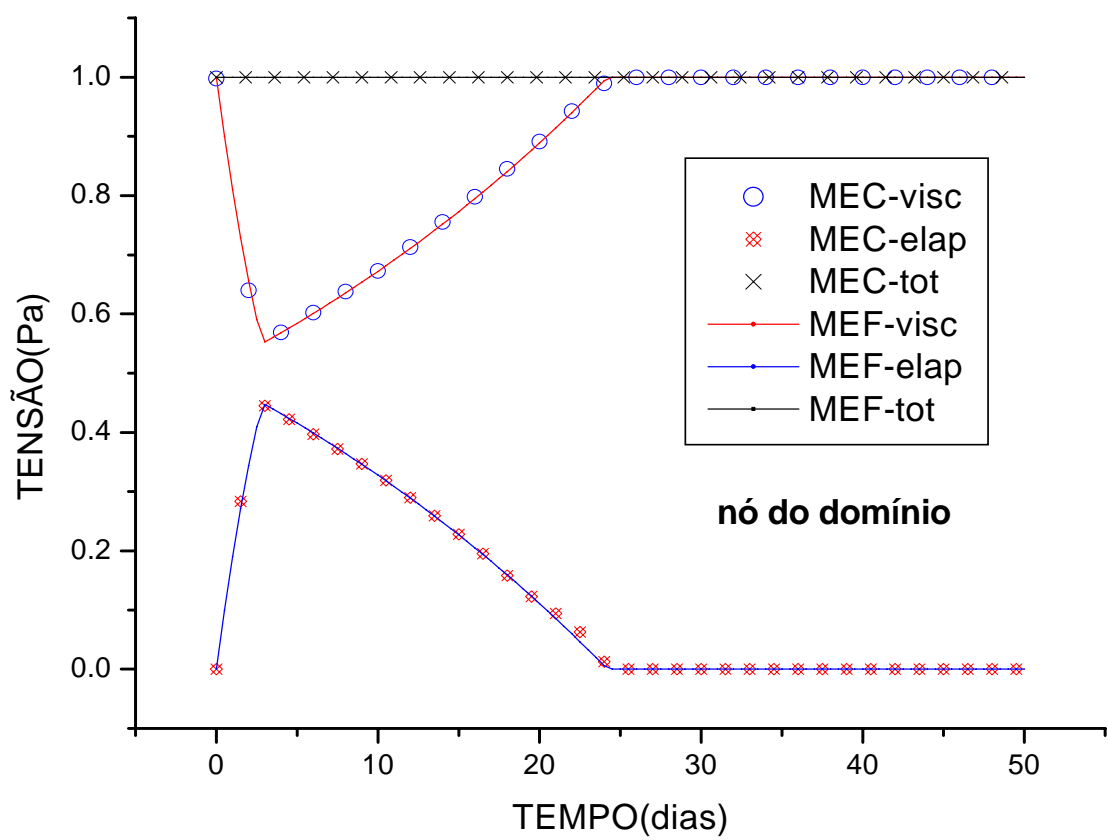

FIGURA 9.12 - Tensões elastoplástica, viscosa e total para o ponto $B$ - encruamento negativo. 


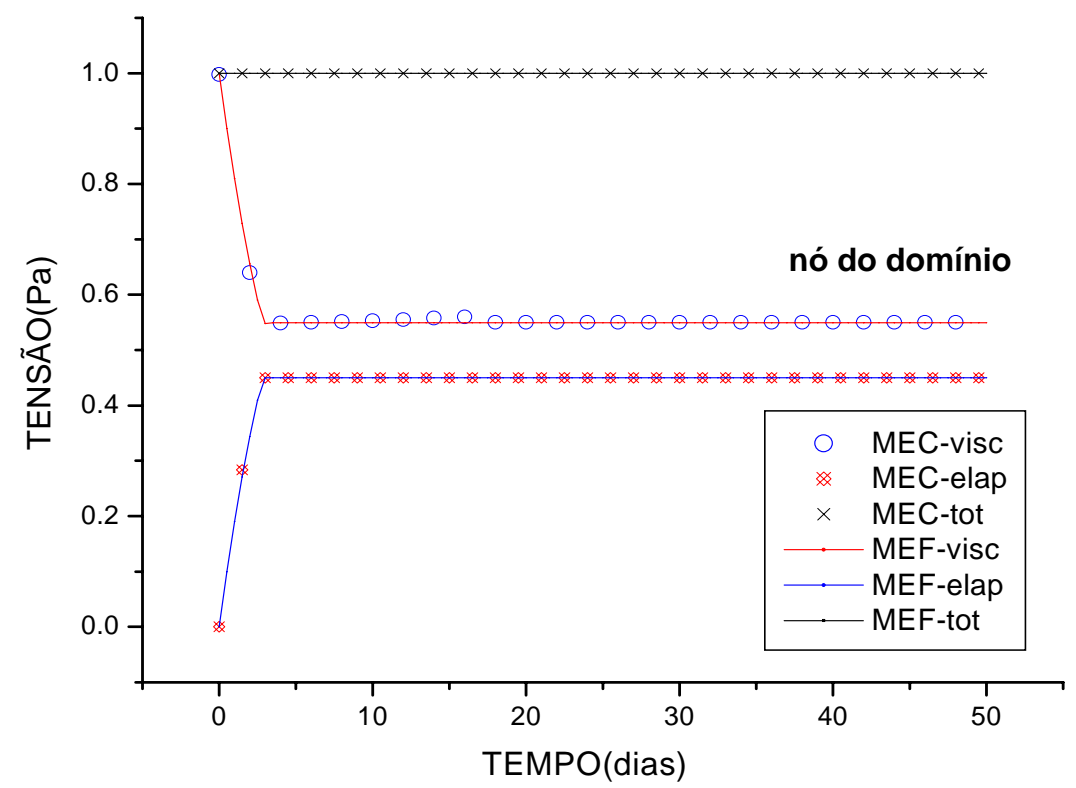

FIGURA 9.13 - Tensões elastoplástica, viscosa e total para o ponto $B$ - plasticidade perfeita.

Observa-se no exemplo apresentado que os resultados obtidos com os dois métodos para os deslocamentos e para as tensões possuem boa concordância entre si. Salienta-se também, que estes resultados estão iguais aos obtidos por MESQUITA (2002) que empregou o MEC não associativo com o critério de Von Mises 3D.

\subsection{EXEMPLO 2 - CHAPA ORTOTRÓPICA}

A chapa a ser analisada é a mesma apresentada na Figura 8.1. A diferença em relação à análise feita no item 8.1 é a consideração da viscoplasticidade e da ortotropia. Assim, têm-se novos parâmetros na análise:

TABELA 9.2 - Parâmetros elásticos e de viscoplasticidade da chapa ortotrópica.

\begin{tabular}{ccc}
\hline Parâmetros & Nomenclatura & Valor \\
\hline Módulo de Young na direção global x & $\mathrm{E}_{\mathrm{x}}$ & $1,0 \mathrm{~Pa}$ \\
Módulo de Young na direção global y & $\mathrm{E}_{\mathrm{y}}$ & $0,5 \mathrm{~Pa}$ \\
Módulo de elasticidade transversal & $\mathrm{G}_{\mathrm{xy}}$ & $0,5 \mathrm{~Pa}$ \\
Coeficiente de Poisson & $v_{\mathrm{xy}}$ & 0,25 \\
Tamanho do incremento de tempo & $\Delta \mathrm{t}$ & $1 \mathrm{dia}$ \\
Número de incrementos de tempo & - & 450 \\
Parâmetro de viscosidade & $\gamma$ & 45,4545 dias \\
Carregamento aplicado & $\mathrm{P}_{\mathrm{x}}$ & $0,005 \mathrm{~Pa}$ \\
Tensão de escoamento & $\sigma_{0}$ & $0,0025 \mathrm{~Pa}$ \\
Módulo de elasticidade tangente & $\mathrm{Et}^{+}$ & $+0,50 \mathrm{E}_{\mathrm{x}}$ \\
\hline
\end{tabular}


Para os pontos $\boldsymbol{A}$ e $\boldsymbol{B}$ da Figura 8.1, considerando-se um encruamento positivo, tem-se as seguintes soluções:

\section{Ponto A:}

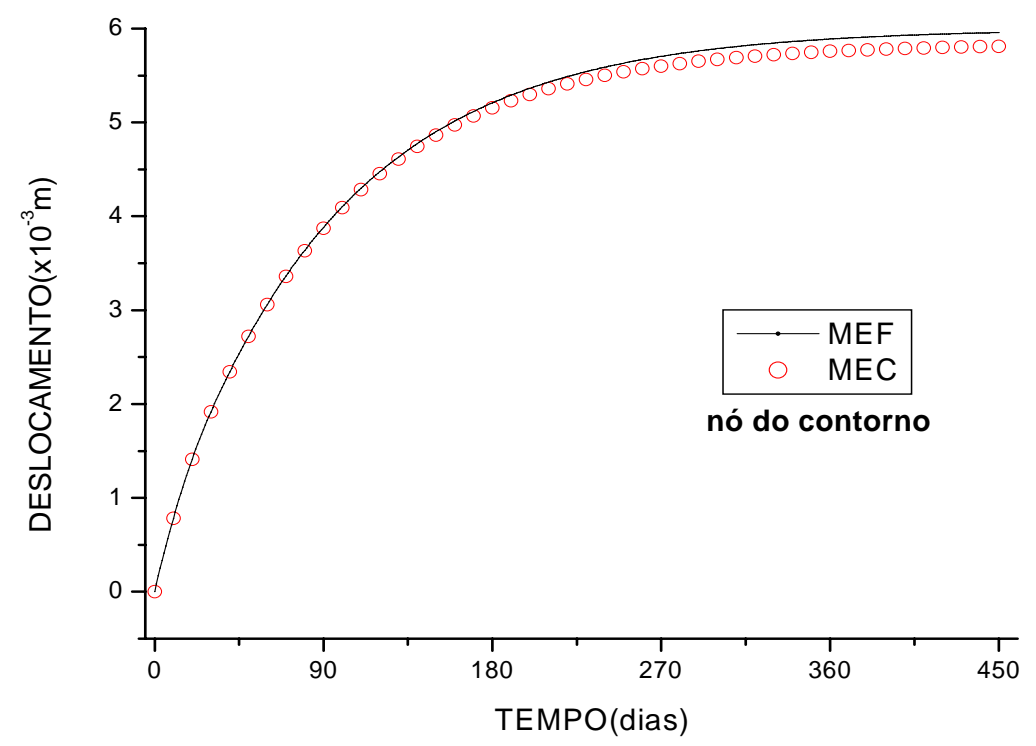

FIGURA 9.14 - Deslocamentos para o ponto A do contorno - MEC e MEF.

e para o ponto $\boldsymbol{B}$ pertencente ao domínio:

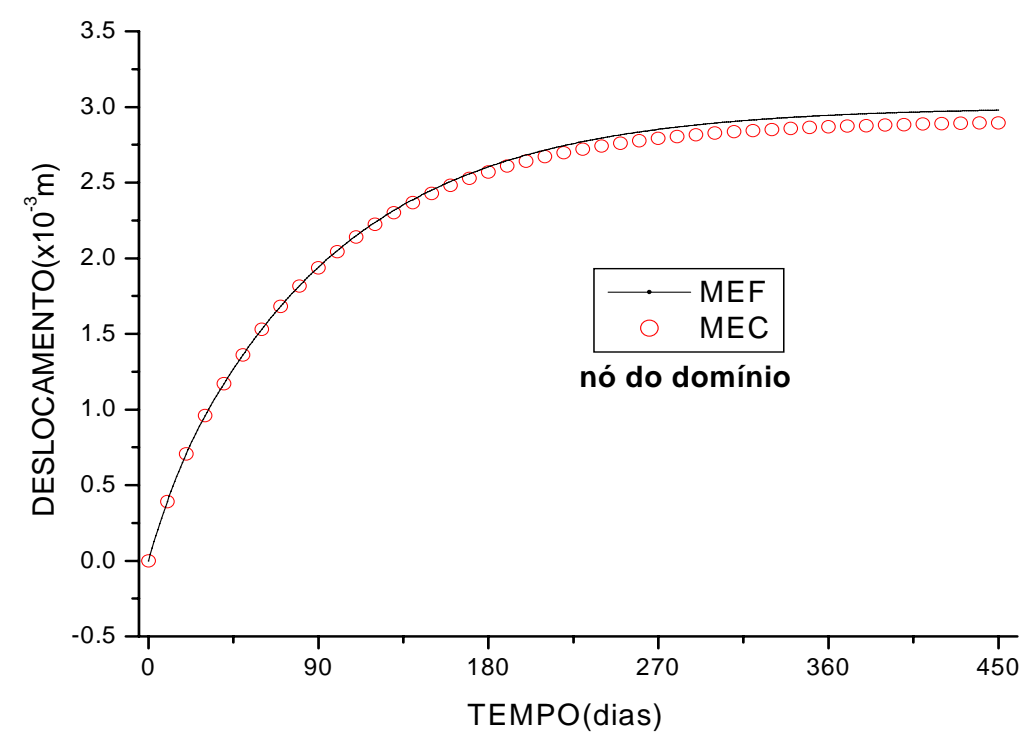

FIGURA 9.15 - Deslocamentos para o ponto $B$ do domínio - MEC e MEF.

Para as tensões normais (elastoplástica, viscosa e total) no ponto $\boldsymbol{A}$ : 


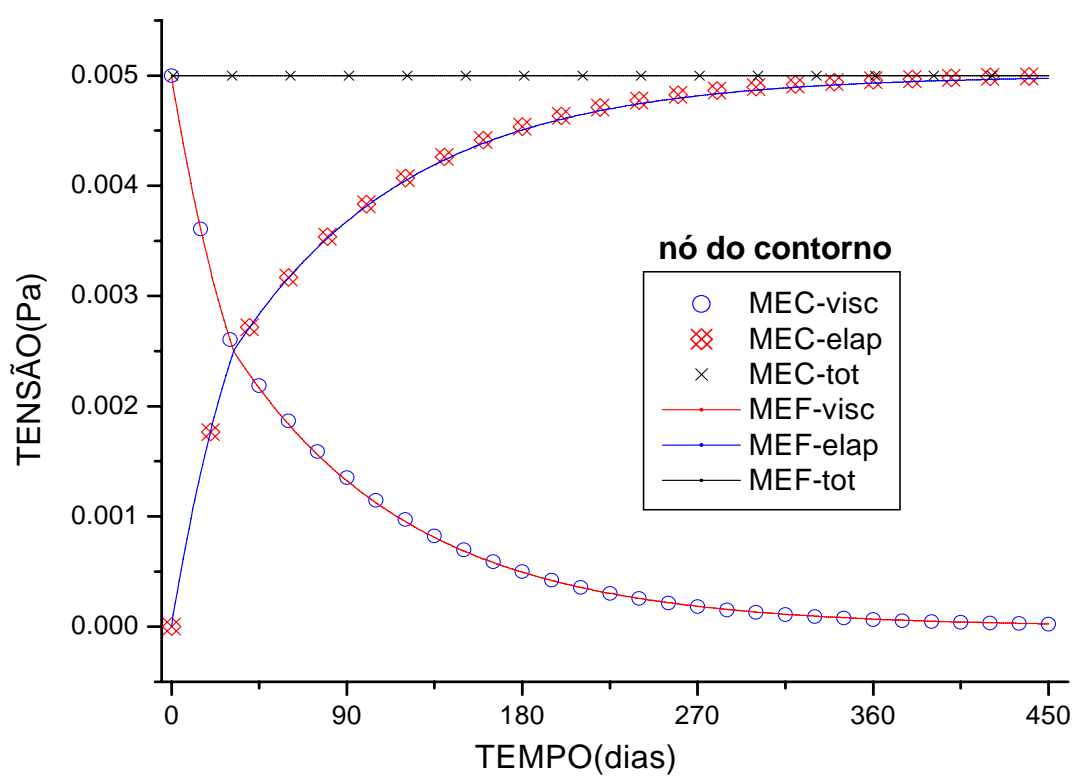

FIGURA 9.16 - Tensões elastoplásticas, viscosa e total para o ponto A - MEC e MEF.

e para o ponto $\boldsymbol{B}$ :

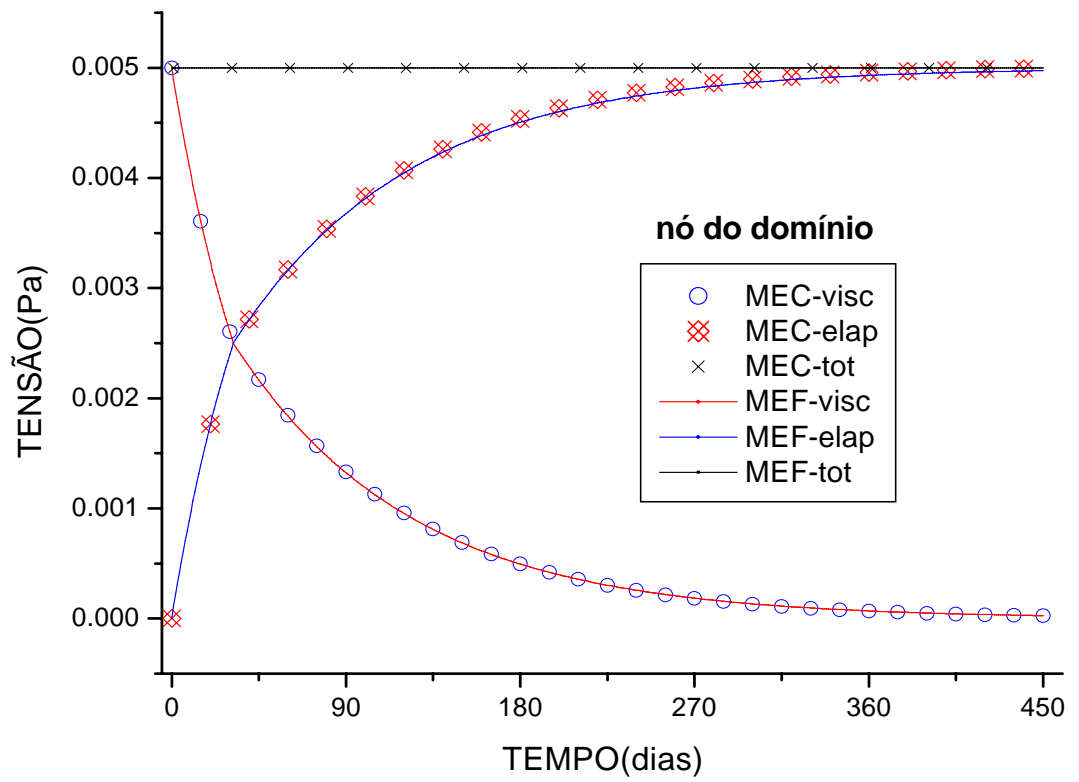

FIGURA 9.17 - Tensões elastoplásticas, viscosa e total para o ponto $B$ - MEC e MEF.

Nas soluções apresentadas, considerou-se, como nos exemplos anteriores, a direção de retorno do fluxo plástico igual à direção do fluxo elástico, utilizando-se para o tensor constitutivo de fluxo plástico um coeficiente de Poisson igual a 0,25. Assim, considera-se que o material da chapa comporta-se como um material compressível conferindo variação de volume no seu processo de plastificação. 
Para a verificação deste fato, no gráfico da Figura 9.18, está apresentada a soma das deformações plásticas principais $\varepsilon_{11}^{p}$ e $\varepsilon_{22}^{p}$, para o ponto $B$, considerando o estado plano de deformação, indicando-se assim, uma variação de volume presente na análise. No mesmo gráfico pode ser vista a soma das deformações principais, para o mesmo exemplo, porém, com um coeficiente de Poisson plástico de 0,5, indicando-se assim, para essa nova situação, uma incompressibilidade (inexistência de variação de volume).

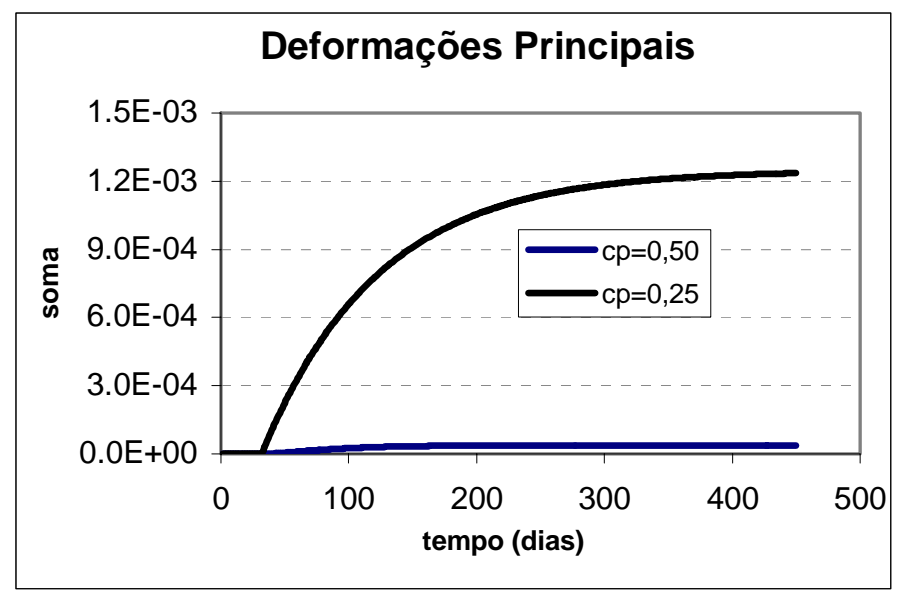

FIGURA 9.18 - Soma das deformações plásticas principais $\varepsilon_{11}^{p}$ e $\varepsilon_{22}^{p}$ - ponto $B$.

\subsection{EXEMPLO 3 - CHAPA COM ANISOTROPIA GERAL}

A chapa a ser analisada está apresentada na Figura 9.19:

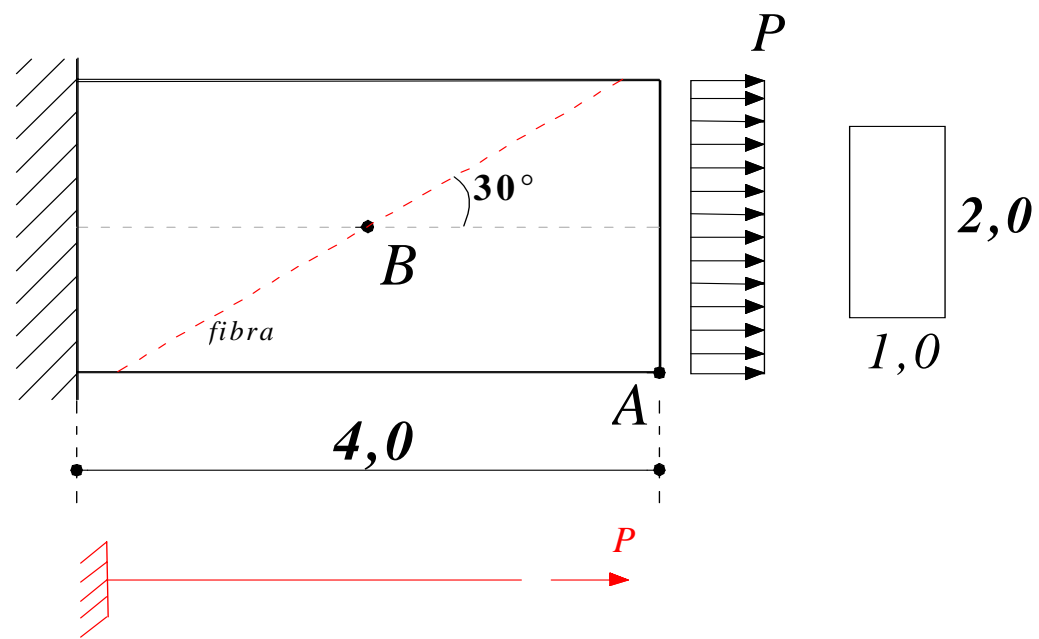

FIGURA 9.19 - Chapa com dimensões de 4m x 2m.

As propriedades elásticas ortotrópicas, em relação ao sistema local das fibras, do material laminado reforçado que compõe a chapa, estão apresentadas na Tabela 9.3: 
TABELA 9.3 - Parâmetros elásticos ortotrópicos do material laminado.

\begin{tabular}{ccc}
\hline Parâmetros & Nomenclatura & Valor \\
\hline Módulo de Young na direção local 1 & $\mathrm{E}_{1}$ & $100000 \mathrm{~Pa}$ \\
Módulo de Young na direção local 2 & $\mathrm{E}_{2}$ & $50000 \mathrm{~Pa}$ \\
Módulo de elasticidade transversal & $\mathrm{G}_{12}$ & $40000 \mathrm{~Pa}$ \\
Coeficiente de Poisson & $v_{12}$ & 0,25 \\
\hline
\end{tabular}

As fibras estão dispostas com uma orientação de 30 graus $\left(\theta=+30^{\circ}\right)$ em relação ao eixo global $x$ (Figura 9.19). Com isso, a partir das propriedades apresentadas na Tabela 9.3, têm-se as seguintes propriedades elásticas anisotrópicas referidas às direções globais da chapa:

TABELA 9.4 - Constantes elásticas do material reforçado que constitui a lâmina considerada eixos globais.

\begin{tabular}{ccc}
\hline Constantes Elásticas & Nomenclatura & Valor \\
\hline Módulo de Young na direção global x & $\mathrm{E}_{\mathrm{x}}$ & $94.117,64 \mathrm{~Pa}$ \\
Módulo de Young na direção global y & $\mathrm{E}_{\mathrm{y}}$ & $63.999,99 \mathrm{~Pa}$ \\
Módulo de elasticidade transversal & $\mathrm{G}_{\mathrm{xy}}$ & $30.769,23 \mathrm{~Pa}$ \\
Coeficiente de Poisson & $v_{\mathrm{xy}}$ & 0,060 \\
Coeficiente de influência mútua (1 ${ }^{\mathrm{a}}$.espécie) & $\eta_{\mathrm{xy}, \mathrm{x}}$ & $-0,204$ \\
Coeficiente de influência mútua (2 ${ }^{\mathrm{a}}$.espécie) & $\eta_{\mathrm{xy}, \mathrm{y}}$ & $-0,416$ \\
\hline
\end{tabular}

As diferentes resistências ao escoamento utilizadas, critério de TSAI \& WU (1971), nas duas direções principais da lâmina, podem ser verificadas na Tabela 9.5:

TABELA 9.5 - Resistências da lâmina ao escoamento.

\begin{tabular}{ccc}
\hline Resistências & Nomenclatura & Valor (Pa) \\
\hline Resistência à tração na direção 1 & $X_{1}^{+}$ & 0,85 \\
Resistência à compressão na direção 1 & $X_{1}^{-}$ & 0,70 \\
Resistência à tração na direção 2 & $X_{2}^{+}$ & 0,50 \\
Resistência à compressão na direção 2 & $X_{2}^{-}$ & 0,70 \\
Resistência positiva ao cisalhamento & $X_{s}^{+}$ & 0,50 \\
Resistência negativa ao cisalhamento & $X_{s}^{-}$ & 0,50 \\
\hline
\end{tabular}

E também, os demais parâmetros necessários para a análise viscoplástica: 
TABELA 9.6 - Parâmetros para a análise viscoplástica.

\begin{tabular}{ccc}
\hline Parâmetros & Nomenclatura & Valor \\
\hline Tamanho do incremento de tempo & $\Delta \mathrm{t}$ & $0,01 \mathrm{dia}$ \\
Número de incrementos de tempo & - & 5000 \\
Parâmetro de viscosidade & $\gamma$ & $4,5 \mathrm{dias}$ \\
Carregamento aplicado & $\mathrm{P}_{\mathrm{x}}$ & $1,0 \mathrm{~Pa}$ \\
Módulo de elasticidade tangente & $\mathrm{E}_{\mathrm{t}}$ & $+0,15 \mathrm{E}_{\mathrm{x}}$ \\
\hline
\end{tabular}

É utilizada, na análise pelo MEF, a mesma discretização apresentada na Figura 9.1 (4 x 4 elementos). Com o MEC, são também empregados 2 elementos de contorno na direção $x$ e 2 na direção $y$, com um número de células igual ao número de elementos finitos usados com o MEF. As soluções são sempre apresentadas para os pontos $A$ e $B$ da Figura 9.19, considerando encruamento positivo e também plasticidade perfeita. Para os deslocamentos no ponto $A$ do contorno tem-se:

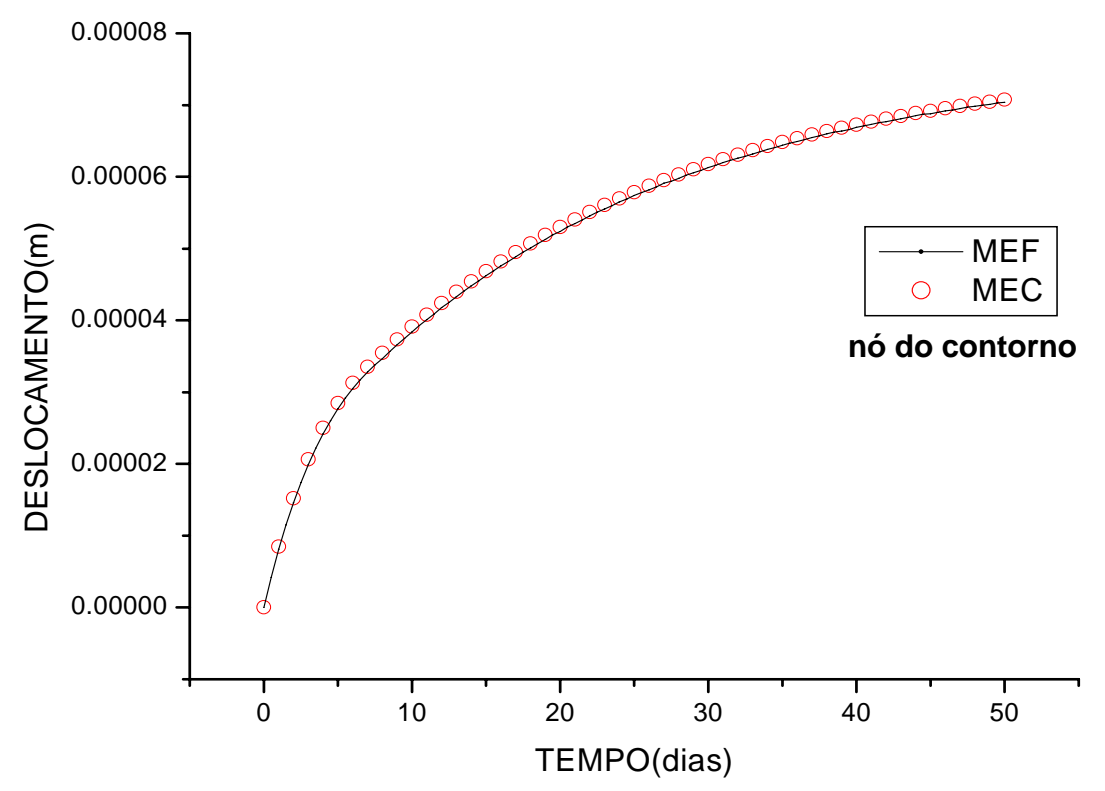

FIGURA 9.20 - Deslocamentos para o ponto A do contorno - MEC e MEF - encruamento. 


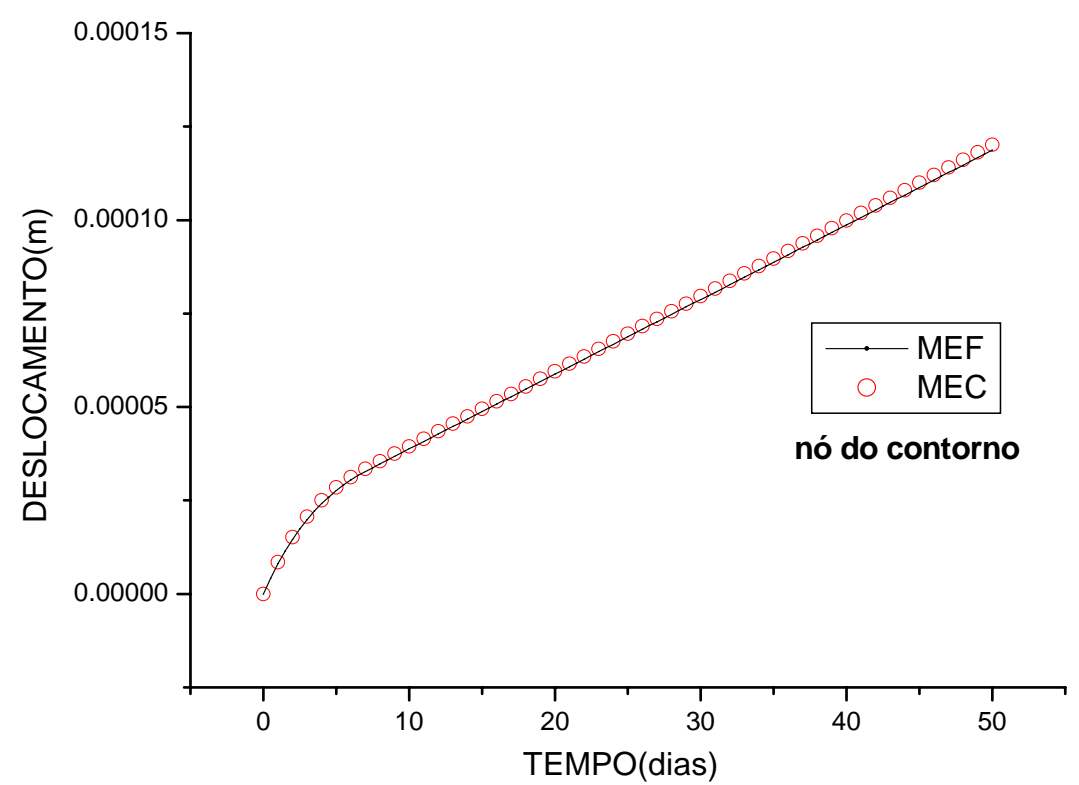

FIGURA 9.21 - Deslocamentos para o ponto A do contorno - MEC e MEF - plasticidade perfeita.

e para o ponto $\boldsymbol{B}$ pertencente ao domínio:

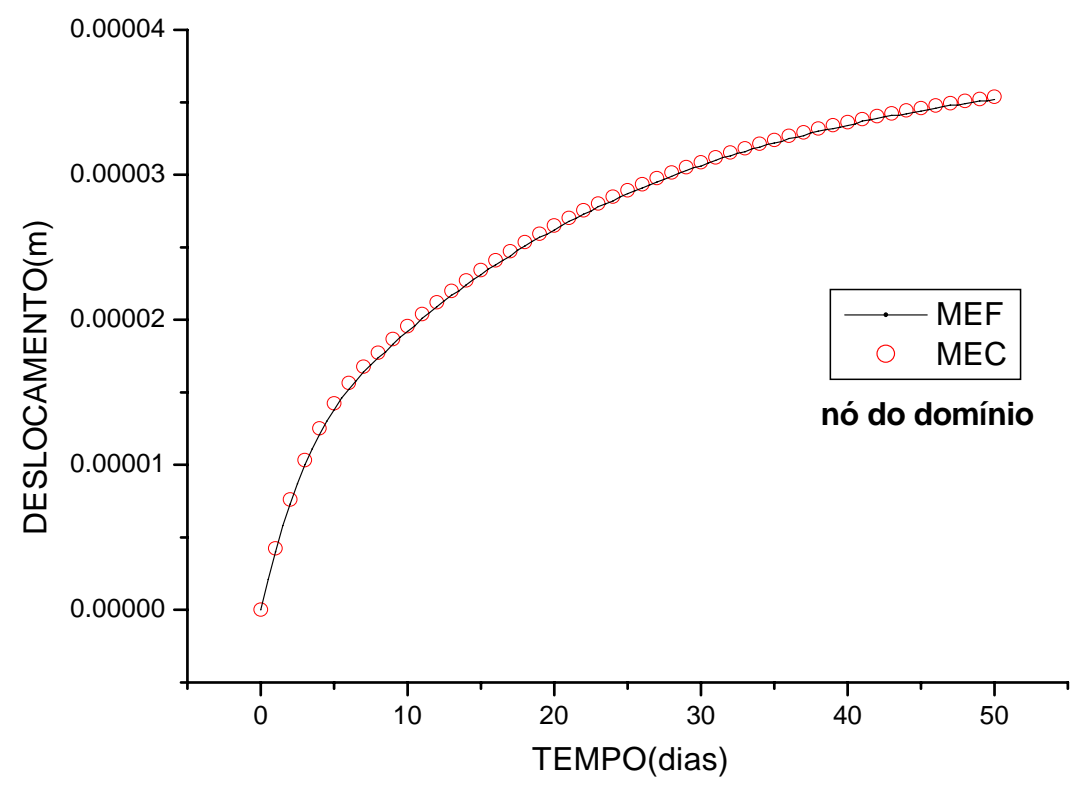

FIGURA 9.22 - Deslocamentos para o ponto $B$ do domínio - MEC e MEF - encruamento. 


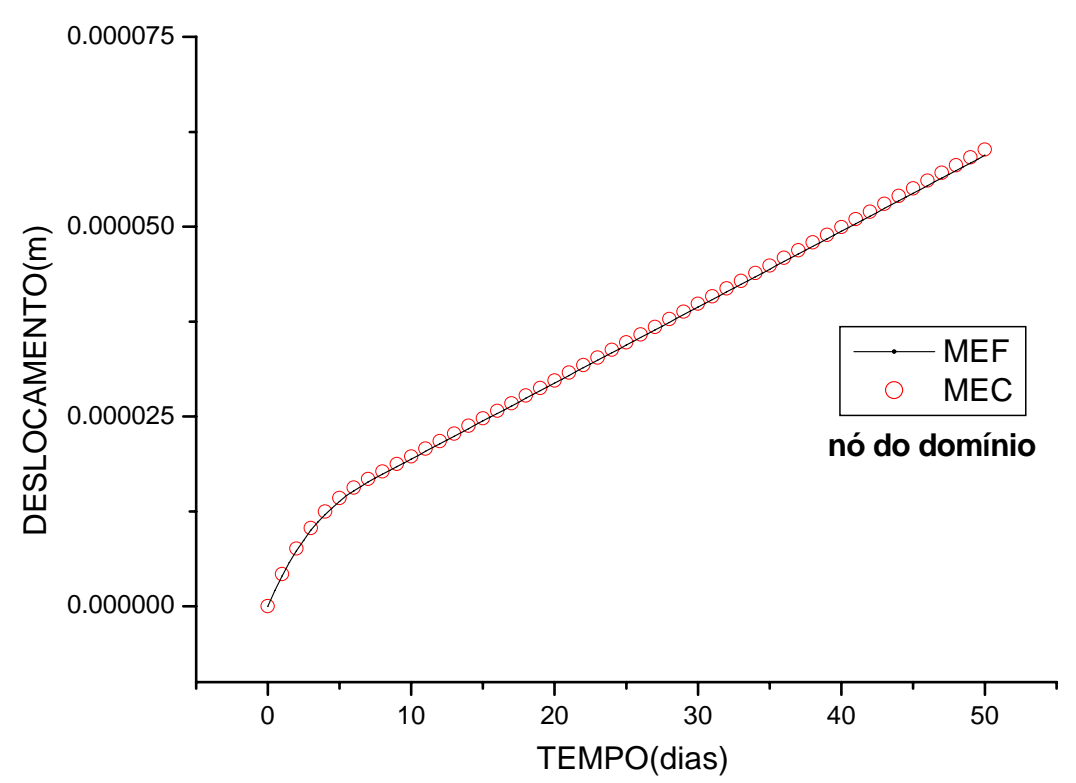

FIGURA 9.23 - Deslocamentos para o ponto B do domínio - MEC e MEF - plasticidade perfeita.

Para as tensões normais (elastoplástica, viscosa e total) no ponto $\boldsymbol{A}$ :

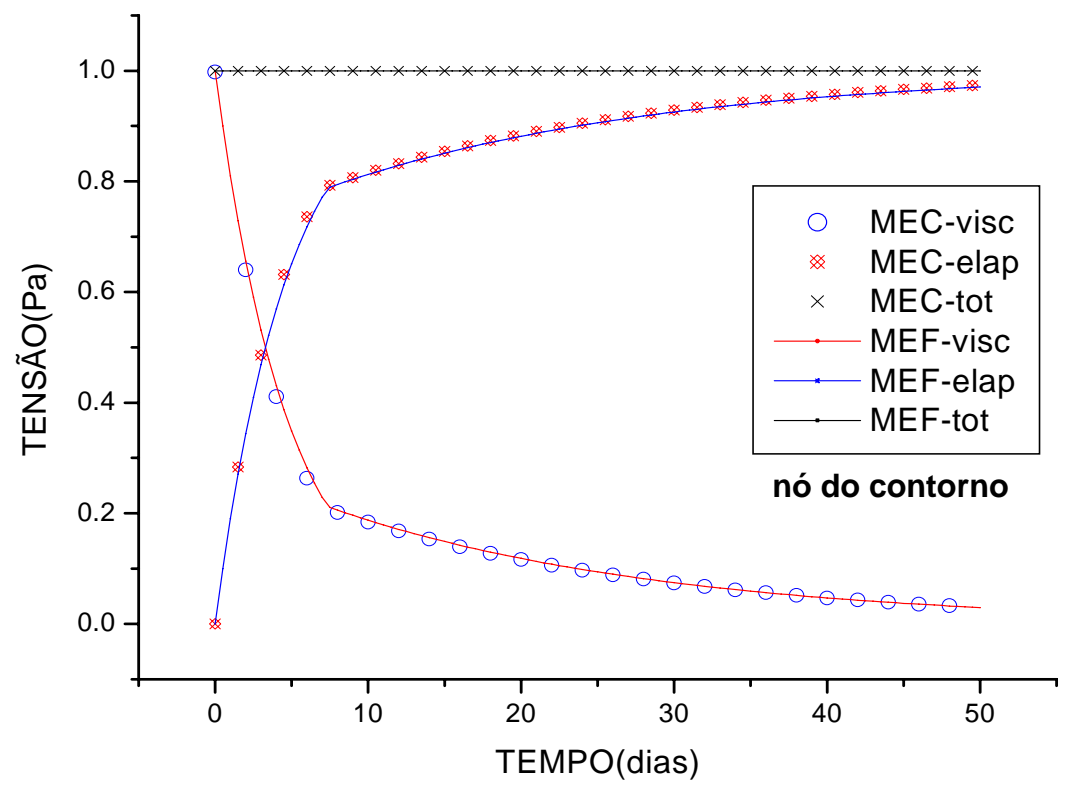

FIGURA 9.24 - Tensões elastoplásticas, viscosa e total para o ponto A - MEC e MEF encruamento. 


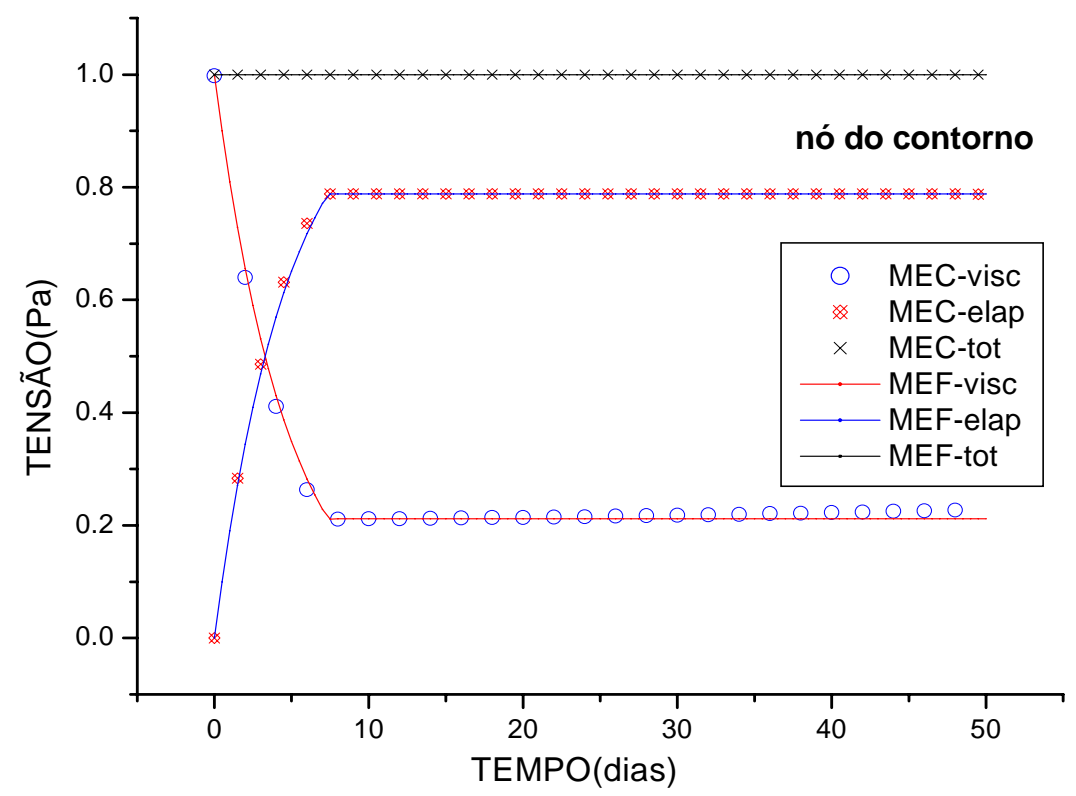

FIGURA 9.25 - Tensões elastoplásticas, viscosa e total para o ponto $A$ - MEC e MEF plasticidade perfeita.

e para o ponto $\boldsymbol{B}$ :

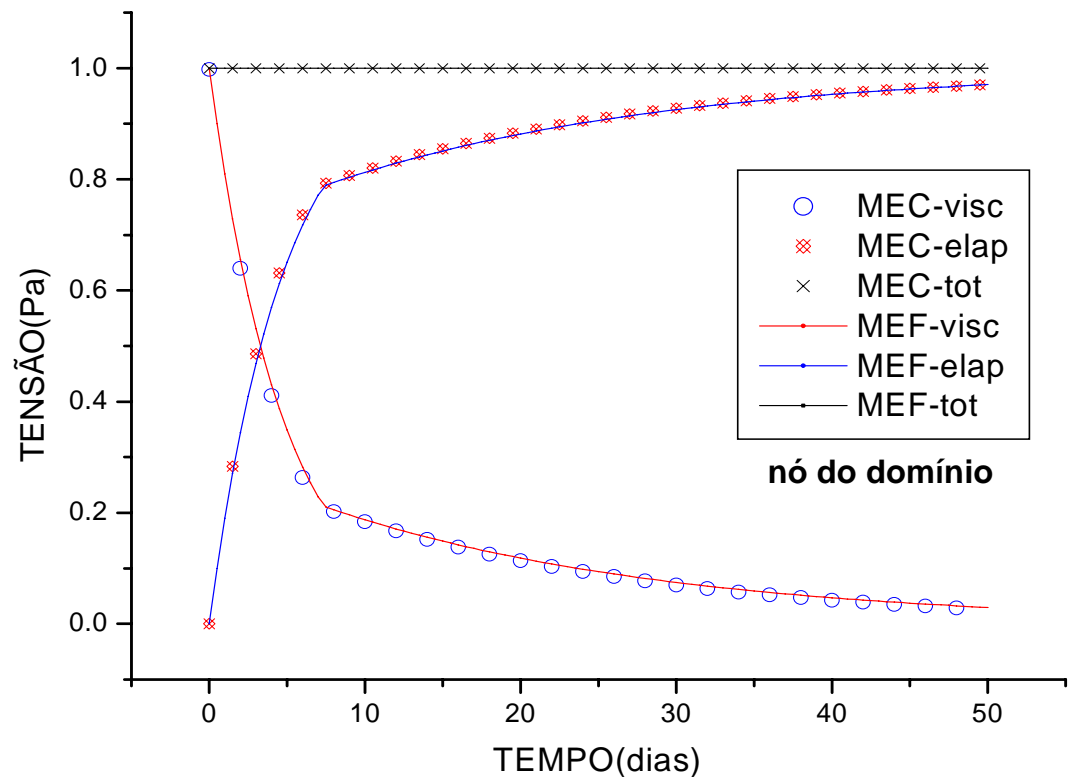

FIGURA 9.26 - Tensões elastoplásticas, viscosa e total para o ponto $B$ - MEC e MEF encruamento. 


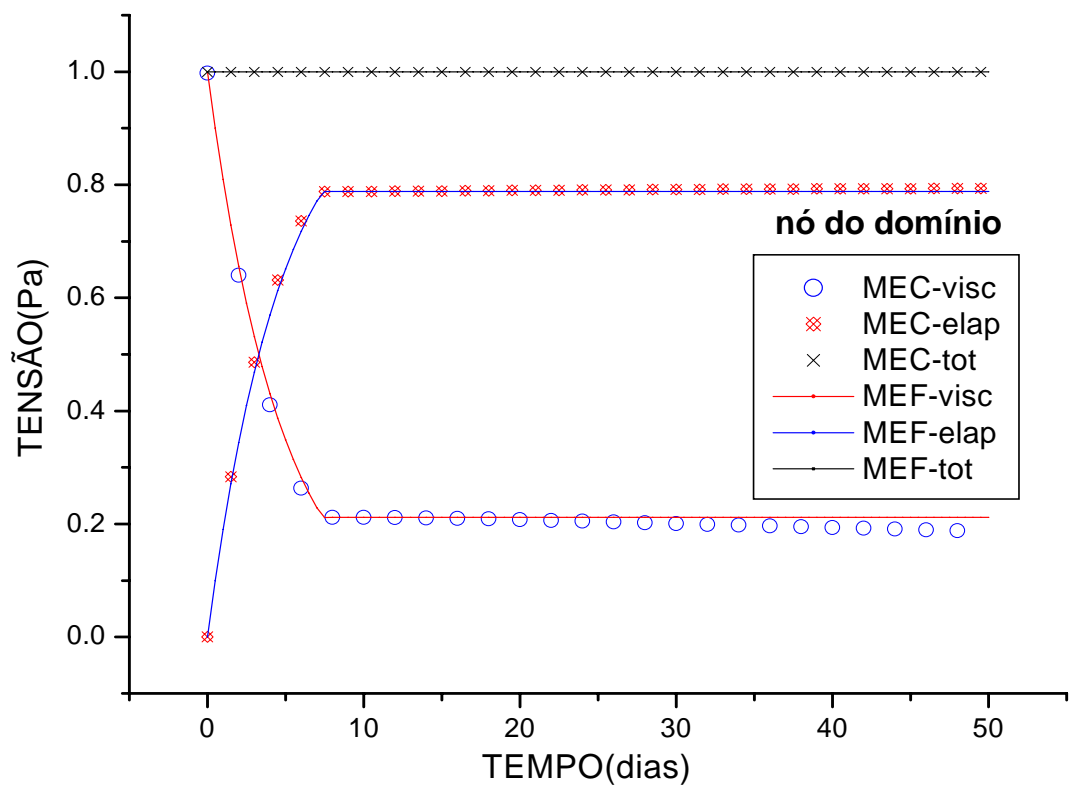

FIGURA 9.27 - Tensões elastoplásticas, viscosa e total para o ponto B - MEC e MEF plasticidade perfeita.

Com a apresentação deste exemplo anisotrópico, finaliza-se este capítulo de aplicações viscoplásticas das formulações desenvolvidas. Deve-se comentar que a metodologia viscoplástica diferencial aplicada a materiais anisotrópicos, tanto utilizando o MEF quanto o MEC é original. O tratamento da plasticidade anisotrópica via MEC é contribuição também original deste trabalho, abrindo mais uma frente de aplicações para esse método numérico. 


\section{MEIOS REFORÇADOS COM FIBRAS - ANÁLISE UTILIZANDO-SE O MEF}

No presente capítulo são analisados meios reforçados com fibras apresentandose, de uma forma geral, aspectos teóricos relacionados com esses materiais, bem como, a formulação numérica desenvolvida para o MEF que permite a consideração de distribuição aleatória de fibras no domínio do problema enfocado, sem qualquer reordenação de malha e nem aumento dos graus de liberdade da análise.

Neste ponto deve-se ressaltar que uma linha de pesquisa importante do SET/EESC é o desenvolvimento de modelos para materiais reforçados através do acoplamento MEC/MEF, como pode ser visto, por exemplo, nos trabalhos de CODA \& VENTURINI (1999), CODA (2000) e LEITE et al. (2003). Nesses trabalhos, a introdução de fibras no contínuo acarreta acréscimo de graus de liberdade no sistema de equações a ser resolvido. Além disso, a necessidade de células internas para o tratamento da não linearidade física da matriz (material) reduz as vantagens do MEC em relação ao MEF.

A técnica aqui proposta resulta em procedimento do MEF mais econômico que o MEC e mais genérico do que aqueles encontrados na literatura especializada do MEF. Até onde se tem conhecimento, também é uma contribuição original deste trabalho.

No final do capítulo, são apresentados alguns exemplos onde se procurou comparar os resultados numéricos com resultados experimentais obtidos na literatura, mostrando-se assim, a eficiência da técnica desenvolvida. 


\subsection{GENERALIDADES}

O desenvolvimento de materiais compósitos reforçados tem ocorrido muito acentuadamente nos últimos anos proporcionando o aparecimento de novas alternativas para os projetos de Engenharia.

Segundo CALLISTER (1985), um material multifase exibindo uma combinação de propriedades que o torna superior a cada fase de sua composição pode ser considerado um compósito.

De acordo com esse princípio de ação combinada, na confecção de um meio compósito procura-se moldar os melhores arranjos de propriedades por uma combinação de dois ou mais materiais distintos, que podem ser classificados como aglomerante (fase contínua ou matriz) ou reforçante (fase dispersa). A matriz tem como função manter os reforçantes unidos, transmitindo a estes o carregamento aplicado. Os reforçantes têm como função suportar os carregamentos transmitidos pela matriz (TITA, 2000).

Devido a grande variedade de aglomerantes e reforçantes, CALLISTER (1985) apresentou uma interessante classificação para os materiais compósitos, reproduzida na Figura 10.1:

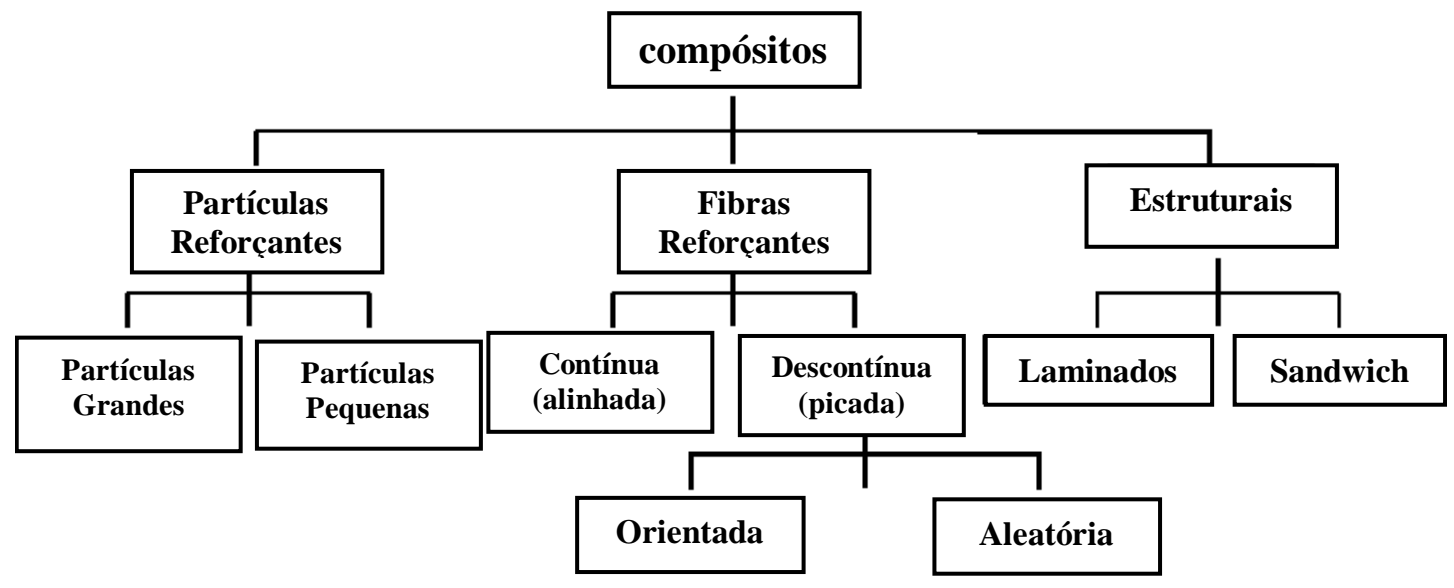

FIGURA 10.1 - Esquema de classificação para materiais compósitos

Pode-se, observando a Figura 10.1, constatar os vários tipos de materiais compósitos existentes, surgindo-se uma gama de aplicações responsável por motivar o interesse de muitos pesquisadores pelo assunto. Neste trabalho, trata-se especificamente da análise numérica plana de meios reforçados por fibras, podendo-se considerar as 
matrizes constituídas de materiais poliméricos (ou cimentícios) e as fibras curtas (ou longas).

Antes de se abordar a formulação numérica empregada expõe-se a seguir uma breve exposição sobre sistemas estruturais com fibras, bem como, alguns trabalhos relacionados ao tema.

\subsection{SISTEMAS COM FIBRAS}

No material compósito a eficiência do reforço com fibras pode ser avaliada de acordo com a melhoria de resistência e de tenacidade do conjunto comparado com a matriz frágil. Com relação às fibras, estas podem ser curtas, de alguns centímetros, ou longas, adicionadas no momento da moldagem, ou cortadas após a fabricação da peça.

Os tipos mais comuns são: fibras de aço, de vidro, de carbono e vegetais. Podem ser definidas como unidirecionais, quando orientadas segundo uma mesma direção; bidimensionais, com as fibras orientadas segundo duas direções ortogonais ou orientadas aleatoriamente; e tridimensionais, quando as fibras são orientadas no espaço tridimensional podendo também ser aleatórias.

As matrizes têm como função principal transferir as solicitações mecânicas às fibras e protegê-las do ambiente externo. As matrizes podem ser cimentícias (concreto ou argamassas), resinosas (polyester, epóxi, etc) e metálicas (ligas de alumínio).

A escolha entre um tipo de fibra e uma matriz depende fundamentalmente da aplicação dada ao material compósito e também do desempenho requerido. O custo, em muitos casos, pode também ser um fator de escolha entre um ou outro componente.

No entanto, as particularidades dos diferentes tipos de fibras e de matrizes introduzem características próprias aos diversos sistemas com fibras, pois, as propriedades mecânicas da fibra, sobretudo o módulo de deformação longitudinal e a resistência, assim como a qualidade da aderência entre a fibra e a matriz, são fatores determinantes do seu desempenho (FURLAN, 1995). Na Tabela 10.1, extraída de BENTUR \& MINDESS (1990), podem ser observadas propriedades para diversos tipos de fibras. 
TABELA 10.1 - Valores de resistência mecânica e módulo de elasticidade para diversos tipos de fibras.

\begin{tabular}{cccccc}
\hline Material & $\begin{array}{c}\text { Diâmetro } \\
(\boldsymbol{\mu m})\end{array}$ & $\begin{array}{c}\text { Densidade } \\
\left(\mathbf{g} / \mathbf{c m}^{3}\right)\end{array}$ & $\begin{array}{c}\text { Módulo de } \\
\text { Elasticidade } \\
(\mathbf{G P a})\end{array}$ & $\begin{array}{c}\text { Resistência } \\
\text { à tração } \\
(\mathbf{M P a})\end{array}$ & $\begin{array}{c}\text { Deformação } \\
\text { na ruptura } \\
(\%)\end{array}$ \\
\hline Aço & $5-500$ & 7,84 & $190-210$ & $0,5-2,0$ & $0,5-3,5$ \\
Vidro & $9-15$ & 2,60 & $70-80$ & $2-4$ & $2-3,5$ \\
Amianto & $0,02-0,4$ & 2,60 & $160-200$ & $3-3,5$ & $2-3$ \\
Polipropileno & $20-200$ & 0,90 & $5-7,7$ & $0,5-0,75$ & 8,0 \\
Kevlar & 10 & 1,45 & $65-133$ & 3,6 & $2,1-4,0$ \\
Carbono & 9 & 1,90 & 230 & 2,6 & 1,0 \\
Nylon & - & 1,10 & 4,0 & 0,9 & $13-15$ \\
Celulose & - & 1,20 & 10 & $0,3-0,5$ & - \\
Acrílico & 18 & 1,18 & $14-19,5$ & $0,4-1,0$ & 3 \\
Polietileno & - & 0,95 & 0,3 & $0,7 \times 10^{-3}$ & 10 \\
Fibra de madeira & - & 1,50 & 71 & 0,9 & - \\
Sisal & $10-50$ & $1-50$ & - & 0,8 & 3,0 \\
Matriz de cimento & - & 2,50 & $10-45$ & $3,7 \times 10^{-3}$ & 0,02 \\
\hline
\end{tabular}

Fonte: BENTUR \& MINDESS (1990)

Na literatura existem muitos trabalhos experimentais e teóricos destinados à abordagem de materiais reforçados com fibras. A seguir, citam-se alguns que foram consultados no intuito de se fundamentar o assunto e também de se colher resultados para comparações com as soluções obtidas com o código implementado.

Salienta-se que um aprofundamento maior sobre o tema não é o interesse principal desta pesquisa, que se especializa no desenvolvimento de um código e de uma formulação do MEF para o tratamento de determinados problemas em meios anisotrópicos reforçados por fibras.

Tratando-se propriamente sobre a mecânica dos materiais compósitos poliméricos reforçados por fibras contínuas, GIBSON (1995) e HYER (1997) apresentam informações detalhadas sobre esses materiais, abordando-se, entre outras coisas, critérios de resistência e análise teórica de tensões.

BENTUR \& MINDESS (1990) abordando compósitos cimentícios reforçados trazem resultados importantes (colhidos de experimentos envolvendo estruturas de concreto reforçado por fibras de aço, de vidro e poliméricas) para aqueles que fazem pesquisas experimentais nesse tema. Em FIGUEIREDO (2000) se encontra uma fundamentação teórica abrangente sobre concreto reforçado com fibras de aço. 
No estudo de cisalhamento em vigas, pesquisas recentes têm mostrado a melhoria do desempenho devido à introdução de fibras curtas de aço em elementos de concreto submetidos a solicitações tangenciais, FURLAN (1995), KHUNTIA et al. (1999), LIM \& OH (1999) e HOLANDA (2002).

De acordo com FURLAN (1995), no concreto as fibras têm sido utilizadas principalmente para vencer algumas de suas limitações: fragilidade, pequena capacidade de deformação e baixa resistência à tração. Geralmente a adição de fibras não visa o aumento de resistência, embora em algumas situações ele ocorra, porém, visa-se o melhor controle da fissuração e o aumento da ductilidade na etapa posterior à fissuração.

Por ser descontínua, a fibra é menos eficiente que a armadura contínua de fios e barras na função de resistir aos esforços de tração e de cisalhamento. No entanto, em função do espaçamento reduzido entre elas, sua atuação como obstáculo ao desenvolvimento de fissuras é superior. Ao interceptar as microfissuras que surgem durante o endurecimento da pasta, as fibras impedem sua progressão e evitam o aparecimento prematuro de macrofissuras. Com isso, a permeabilidade do concreto e a região exposta ao ambiente são menores, melhorando as condições de durabilidade (FURLAN, 1995).

Neste ponto é importante comentar que apesar do grande número de referências experimentais sobre o assunto reforço com fibras, com diferentes enfoques, a quantidade de pesquisas numéricas não é tão grande.

A maioria dos trabalhos existentes apresenta resultados oriundos da utilização de softwares comerciais como ANSYS ${ }^{1}$, ABAQUS ${ }^{2}$ e DIANA ${ }^{3}$, os quais, são de grande potencialidade para os mais diversos tipos de análises de Engenharia, porém, na modelagem de fibras imersas no domínio (AL-ORAIMI \& SEIBI, 1995; DAVIS et al., 2002; HU et al., 2004; COURAGE \& SCHREURS, 1992), não fazem uso da mesma abordagem empregada na presente pesquisa onde, além de se permitir que o reforço (fibras curtas ou longas) seja modelado como um elemento finito elastoplástico de barra

\footnotetext{
${ }^{1}$ ANSYS Manual and Software Version 5.0, Swanson Analysis Systems, Inc. Canonsburg.

${ }^{2}$ Hibbitt, Karlsson and Sorensen, Inc. ABAQUS Theory Manual, User Manual and Example Manual, Version 6.3, Providence, RI, 2002.

${ }^{3}$ DIANA, DIANA finite element analysis user manuals, TNO Institute for Building Materials and Building Structures, Rijswijk (1989).
} 
simples cujos nós não precisam estar coincidentes com os nós da malha onde a fibra se insere, nenhum elemento finito plano especial para meios compósitos precisa ser utilizado. Empregando-se a formulação aqui proposta nenhuma técnica de redimensionamento de malha é necessária, atividade muito comum em softwares comerciais.

Salienta-se também, que na consulta bibliográfica realizada até este momento, em bibliotecas físicas ou digitais, não se encontrou nenhum trabalho que tratasse da modelagem aleatória de fibras pelo MEF, sem aumento dos graus de liberdade do problema e sem coincidência de posicionamento de nós. É preciso dizer que em aplicações do MEC a modelagem do reforço em qualquer posição do domínio é uma característica natural da técnica, como pode ser constatado, por exemplo, no trabalho de CODA (2001) e LEITE et al. (2003).

\subsection{FORMULAÇÃO DO MEF PARA A CONSIDERAÇÃO DO REFORÇO NUMA POSIÇÃO QUALQUER DO DOMÍNIO}

Neste item descreve-se a formulação desenvolvida para o tratamento de problemas planos, pelo MEF, em meios reforçados por fibras. Como já exposto, a característica principal desta formulação é a consideração de distribuição randômica das fibras imersas no meio, sem qualquer aumento dos graus de liberdade do problema analisado.

Desta forma, visando-se a descrição da técnica empregada, parte-se da escrita do problema de maneira energética, de forma simplificada, como:

$$
\Pi=U+\Omega
$$

onde, $\Pi$ é a energia potencial total do sistema, $\Omega$ é a energia potencial das cargas externas e $U$ é a energia de deformação, que por sua vez, é a parcela que dá origem à matriz de rigidez do meio reforçado analisado.

Neste momento, cabe-se observar que as fibras imersas na matriz do sistema analisado funcionam como enrijecedores, aumentando a rigidez total do meio, constituindo, como já comentado, o meio reforçado. Na aproximação numérica por elementos finitos isso significa que a fibra estará contribuindo com a rigidez do 
elemento finito (ou dos elementos finitos) no qual ela está inserida. Desta maneira podese admitir a energia de deformação do problema escrita em duas parcelas:

$$
U=U_{E F}+U_{f}
$$

onde, $U_{E F}$ é a energia de deformação armazenada nos elementos finitos de chapa e $U_{f}$ é a energia de deformação armazenada nas fibras (elementos finitos de barra simples).

A partir deste ponto, pode-se expandir a Equação (10.2) obtendo-se:

$$
U=\frac{1}{2} \int_{\Omega_{\mathrm{EF}}} u_{E F}^{T} \cdot B^{T} \cdot C \cdot B \cdot u_{E F} d \Omega+\frac{1}{2} \int_{\Omega_{f}} u_{f}^{T} \cdot b^{T} \cdot c \cdot b \cdot u_{f} d \Omega
$$

onde, $B$ e $b$ são as matrizes formadas pelas derivadas das funções de forma e que exprimem o relacionamento entre deformações e incógnitas nodais dos elementos finitos de chapa e de barra, respectivamente; $u_{E F}$ e $u_{f}$ são os deslocamentos dos nós dos elementos finitos de chapa e das fibras, respectivamente, e por fim, $C$ e $c$ são os tensores constitutivos dos materiais que constituem matriz e reforço, aqui representados simbolicamente.

Na Equação (10.3) pode-se evidenciar a matriz de rigidez dos dois meios ( $K_{E F}$ : matriz aglomerante, $k_{f}$ : fibra), tendo-se então:

$$
U=\frac{1}{2} u_{E F}^{T} \cdot K_{E F} \cdot u_{E F}+\frac{1}{2} u_{f}^{T} \cdot k_{f} \cdot u_{f}
$$

Neste ponto, é oportuno introduzir a hipótese fundamental da formulação, hipótese essa que apesar de muito simples é original, permitindo que as fibras sejam colocadas em qualquer posição do domínio do problema sem aumento dos graus de liberdade da análise. Para tanto, escreve-se os deslocamentos nodais das fibras em função dos deslocamentos nodais dos nós dos elementos finitos onde elas estão imersas, ou seja:

$$
u_{f}=\phi_{n} \cdot u_{E F}^{n}
$$

sendo que $\phi_{n}$ são as funções de forma dos nós do elemento finito de chapa calculadas para as coordenadas adimensionais do nó de fibra considerado. Assim, pode-se reescrever a Equação (10.4) como: 


$$
U=\frac{1}{2} u_{E F}^{T} \cdot K_{E F} \cdot u_{E F}+\frac{1}{2} \phi_{n}^{T} u_{E F}^{T} \cdot k_{f} \cdot \phi_{n} u_{E F}
$$

e ainda,

$$
U=\frac{1}{2} u_{E F}^{T} \cdot K_{E F} \cdot u_{E F}+\frac{1}{2} u_{E F}^{T} \cdot \bar{k}_{f} \cdot u_{E F}
$$

onde:

$$
\bar{k}_{f}=\phi_{n}^{T} \cdot k_{f} \cdot \phi_{n}
$$

Logo, a energia de deformação do sistema pode ser escrita como:

$$
U=\frac{1}{2} u_{E F}^{T} \cdot\left(K_{E F}+\bar{k}_{f}\right) \cdot u_{E F}
$$

com a matriz de rigidez do meio reforçado sendo composta pelas matrizes de rigidez do elemento finito de chapa - nesta pesquisa sendo utilizado o elemento triangular QST - e do elemento finito de barra simples (do tipo barra de treliça):

$$
K=K_{E F}+\bar{k}_{f}
$$

Com isso, de posse da matriz de rigidez do meio reforçado e utilizando-se o equacionamento para o tratamento de problemas planos pelo MEF apresentado no capítulo 4, pode-se analisar uma grande variedade de problemas práticos que envolvam materiais reforçados com fibras, como por exemplo, análise de tensões e de deslocamentos em vigas de concreto armado, de concreto reforçado com fibras curtas, e em chapas poliméricas reforçados com fibras longas.

Destaca-se também, no código implementado, a possibilidade de se considerar plasticidade nos dois meios (fibra e matriz) de maneira independente com as opções de se utilizar, para a matriz, os critérios de resistência de TSAI \& WU (1971) e Drucker Praguer. É importante ainda comentar que a formulação implementada até o momento não considera o deslizamento das fibras na matriz. No entanto, apesar da ausência dessa característica, muitos problemas podem ser tratados com o código desenvolvido, como comentado anteriormente, e a consideração deste item adicional é completamente factível, porém, objeto de estudos futuros. 
Antes de se apresentar algumas aplicações, é oportuno detalhar um pouco melhor a formação da matriz de rigidez da fibra, Equação (10.8), bem como tecer alguns comentários sobre o programa implementado.

A Equação (10.8) pode ser escrita matricialmente como:

$$
\left[\bar{k}_{f}\right]_{40 \times 40}=\left[\phi_{n}\right]_{40 \times 4}^{T} \cdot\left[k_{f}\right]_{4 \times 4} \cdot\left[\phi_{n}\right]_{4 \times 40}
$$

Lembrando-se que as funções de forma $\phi$, para o presente trabalho, são do elemento finito QST que possui 10 nós. Na constituição da matriz dessas funções, devese prever que uma fibra pode estar inserida num único elemento finito de chapa, ou seja, seus dois nós contidos no mesmo elemento finito, ou pode fazer parte de dois elementos, adjacentes ou não (Figura 10.2).

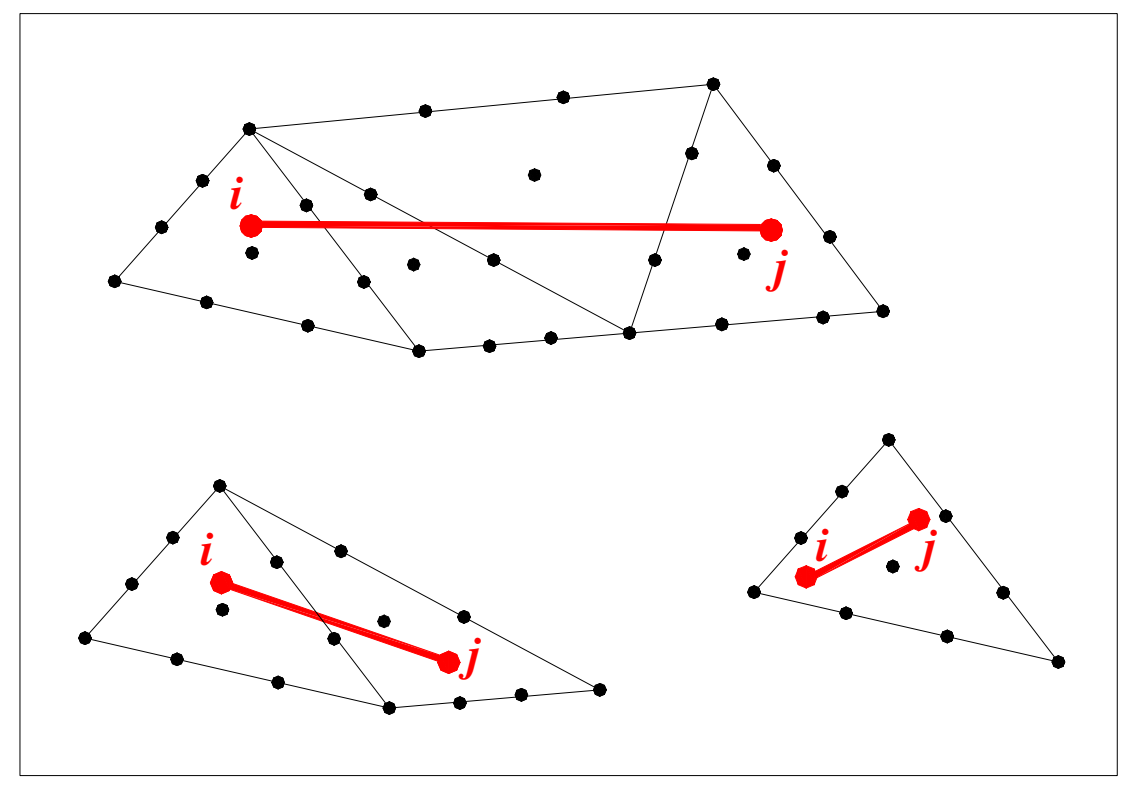

FIGURA 10.2 - Posições possíveis de uma fibra no domínio discretizado em elementos finitos triangulares QST.

Por isso, na implementação realizada, com o intuito de se ter consistência nas operações matriciais, foi preciso organizar a matriz de funções de forma com ordem de (4 x 40) dividindo-a em quatro quadrantes. Dois quadrantes são preenchidos por valores das funções de forma e dois quadrantes preenchidos por zeros, Equação (10.12):

$$
\left[\phi_{n}\right]_{4 \times 40}=\left[\begin{array}{ccccccccccccc}
\phi_{1}^{i} & 0 & \phi_{2}^{i} & \ldots & \phi_{n}^{i} & 0 & \mid & 0 & 0 & 0 & \ldots & 0 & 0 \\
0 & \phi_{1}^{i} & 0 & \ldots & 0 & \phi_{n}^{i} & 0 & 0 & 0 & \ldots & 0 & 0 \\
- & - & - & - & - & - & \mid & - & - & - & - & - & - \\
0 & 0 & 0 & \ldots & 0 & 0 & \mid & \phi_{1}^{j} & 0 & \phi_{2}^{j} & \ldots & \phi_{n}^{j} & 0 \\
0 & 0 & 0 & \ldots & 0 & 0 & \mid & 0 & \phi_{2}^{j} & 0 & \ldots & 0 & \phi_{n}^{j}
\end{array}\right]
$$


Na Equação (10.12), o índice $i$ se refere ao nó inicial da fibra e o índice $j$ ao nó final. Assim, após se efetuar a operação matricial apresentada na Equação (10.11), tem-se uma matriz de rigidez da fibra que deve ser contribuída, de maneira adequada, nas posições referentes ao elemento finito de chapa (ou, elementos finitos), no qual a fibra se insere, da matriz de rigidez global da estrutura.

Sobre o posicionamento das fibras no domínio analisado, o programa permite que esse posicionamento seja aleatório (para fibras curtas ou contínuas) ou definido segundo linhas de fibras longas. Para a geração randômica de nós que formarão as fibras foi utilizado um comando interno do FORTRAN de geração aleatória de pontos, chamado RANDOM. Ao se especificar o comprimento desejado da fibra, são gerados dois pontos onde o primeiro é utilizado como ponto inicial e o segundo como direção (cossenos diretores da fibra) necessária para se formar o comprimento de fibra especificado pelo analista. Essa rotina é repetida de acordo com o número de fibras que se tenha no problema em questão.

Deve-se ressaltar que, para se calcular as funções de forma da matriz (10.12) é preciso se conhecer as coordenadas adimensionais do nó da fibra, pois, na geração aleatória, se conhece apenas as coordenadas cartesianas. Para tanto, utiliza-se um procedimento simples que está exposto com detalhes no Anexo 3.

Tendo-se descrito a formulação empregada, apresenta-se na seção seguinte algumas aplicações do código desenvolvido.

\subsection{APLICAÇÕES DO CÓDIGO DESENVOLVIDO}

No decorrer deste item são apresentados três exemplos visando-se aplicar a formulação desenvolvida neste capítulo. Procurou-se, a partir do segundo exemplo, comparar os resultados numéricos com resultados experimentais colhidos na literatura. Todos os resultados mostram com boa reprodução os comportamentos mecânicos dos exemplos analisados. 


\subsubsection{Análise de Chapas Reforçadas em Regime Elástico-Linear}

Este primeiro exemplo apresenta três análises lineares envolvendo chapas anisotrópicas tracionadas reforçadas com fibras longas. Com estas aplicações, mostra-se as possibilidades de utilização do código na consideração de chapas com a presença de reforço, ou seja, com a presença da fibra imersa no domínio discretizado em elementos finitos de chapa. Na análise, para se modelar as matrizes, são usados (4 x 4) elementos finitos QST, semelhante ao que se apresenta na Figura 9.1, do capítulo 9.

\subsubsection{Chapa ortotrópica - fibras dispostas unidirecionalmente}

A chapa analisada está apresentada na Figura 10.3:

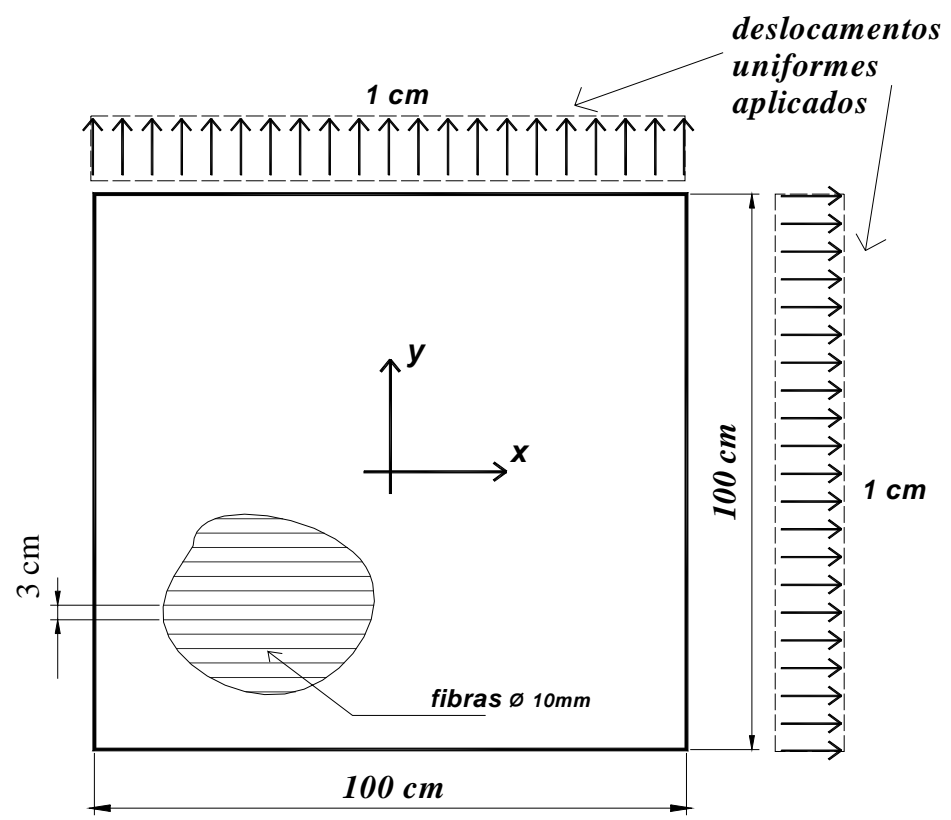

FIGURA 10.3 - Chapa reforçada unidirecionalmente com barras de $10 \mathrm{~mm}$

Como reforço a chapa possui fibras de aço com diâmetro de $10 \mathrm{~mm}$ (área de $0,785 \mathrm{~cm}^{2}$ ) dispostas na direção do eixo $x$, com um espaçamento de $3 \mathrm{~cm}$ entre elas. $\mathrm{O}$ material que constitui a matriz pode ser qualquer, mas, neste exemplo, se considera a matriz como cimentícia. As propriedades elásticas dos dois meios estão na Tabela 10.2: 
TABELA 10.2 - Propriedades dos materiais que constituem o meio compósito analisado.

\begin{tabular}{ccccc}
\hline \multirow{2}{*}{ Material } & \multicolumn{4}{c}{ Propriedades elásticas (direções locais do material) } \\
\cline { 2 - 5 } & $E_{1}(\mathrm{MPa})$ & $E_{2}(\mathrm{MPa})$ & $v$ & $G_{12}(\mathrm{MPa})$ \\
\hline Fibras de aço & $210.000,0$ & $210.000,0$ & 0,3 & $80.000,0$ \\
\hline Matriz cimentícia & $20.000,0$ & $20.000,0$ & 0,2 & $8.333,0$ \\
\hline
\end{tabular}

A finalidade principal da análise é a determinação das propriedades elásticas desse meio compósito ortotrópico plano. Para tanto, são aplicados separadamente deslocamentos uniformes nas duas direções ortogonais ( $x$ e $y$ ) da chapa, podendo-se assim, com as respostas numéricas de deformações e de tensões fornecidas pelo programa, se obter as propriedades do sistema. As respostas são mostradas na Tabela 10.3:

TABELA 10.3 - Propriedades elásticas globais do meio compósito da Figura 10.3.

\begin{tabular}{cccccc}
\hline \multirow{2}{*}{ Material } & \multicolumn{5}{c}{ Propriedades elásticas (direções globais) } \\
\cline { 2 - 6 } & $E_{x}(\mathrm{MPa})$ & $E_{y}(\mathrm{MPa})$ & $v_{x y}$ & $v_{y x}$ & $G_{x y}(\mathrm{MPa})$ \\
\hline $\begin{array}{c}\text { Chapa } \\
\text { compósita }\end{array}$ & $31.214,08$ & $20.291,51$ & 0,20 & 0,13 & $13.005,86$ \\
\hline
\end{tabular}

Este exemplo simples evidencia uma característica importante do código desenvolvido, que é a de se permitir a determinação numérica de propriedades elásticas de certos materiais fibrosos empregados em ensaios de laboratório como, por exemplo, chapas de materiais poliméricos reforçados ou de madeira laminada. A simulação de chapas reforçadas com partículas (fibras curtas) pode também ser efetuada.

\subsubsection{Chapa ortotrópica - fibras dispostas em duas direções ortogonais}

A chapa analisada está apresentada na Figura 10.4: 


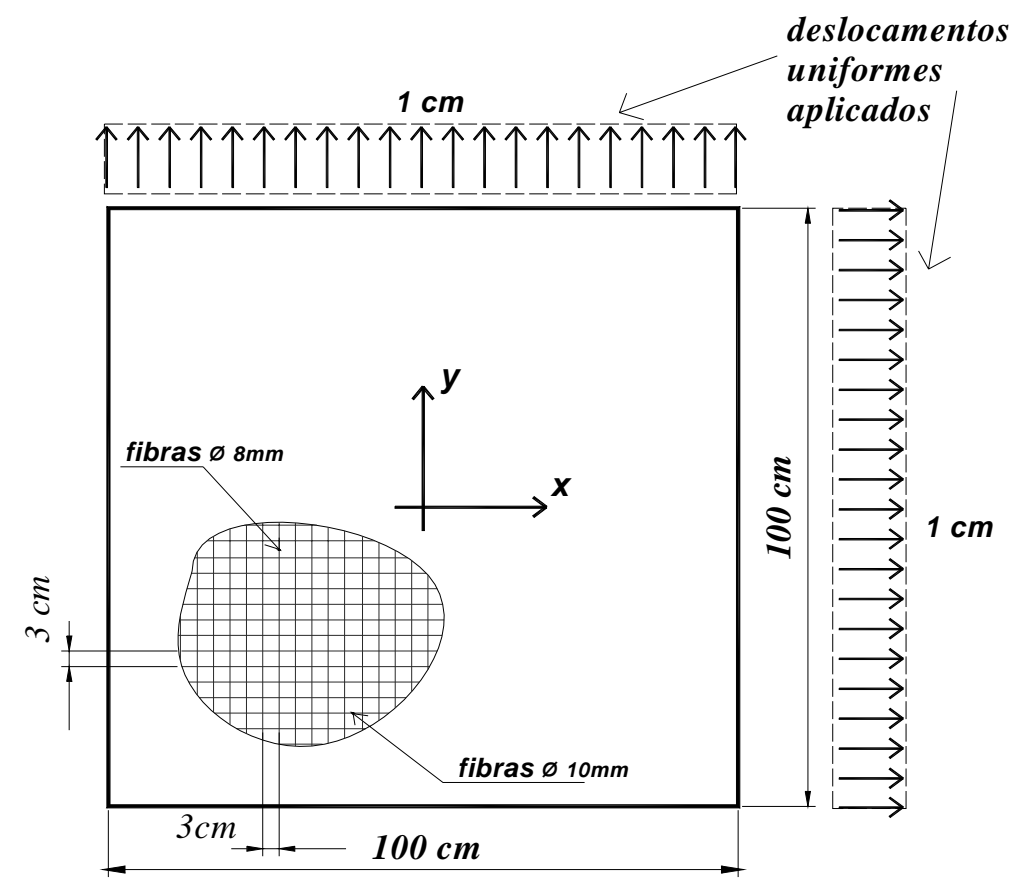

FIGURA 10.4 - Chapa reforçada em duas direções, barras de 10mm e de 8mm.

Esta aplicação é similar a anterior, porém, são consideradas fibras, de diâmetros diferentes, em duas direções ortogonais (eixos $x$ e $y$ ) da chapa. As propriedades isoladas de cada meio são mostradas na Tabela 10.4:

TABELA 10.4 - Propriedades elásticas das fibras e da matriz.

\begin{tabular}{ccccc}
\hline \multirow{2}{*}{ Material } & \multicolumn{4}{c}{ Propriedades elásticas (direções locais do material) } \\
\cline { 2 - 5 } & $E_{1}(\mathrm{MPa})$ & $E_{2}(\mathrm{MPa})$ & $v$ & $G_{12}(\mathrm{MPa})$ \\
\hline Fibra de $10 \mathrm{~mm}$ & $210.000,0$ & $210.000,0$ & 0,3 & $80.000,0$ \\
\hline Fibra de $8 \mathrm{~mm}$ & $150.000,0$ & $150.000,0$ & 0,3 & $62.500,0$ \\
\hline Matriz cimentícia & $20.000,0$ & $20.000,0$ & 0,2 & $8.333,0$ \\
\hline
\end{tabular}

Como feito no item 10.4.1.1, são impostos deslocamentos nas duas direções globais da chapa ( $x$ e $y$ ) visando-se a realização do ensaio numérico. É importante comentar, que para a disposição das fibras no meio, não se preocupou com a coincidência no posicionamento entre os nós das fibras e dos elementos finitos de chapa, aproveitando-se assim, da característica permitida pela formulação. As propriedades obtidas na simulação estão mostradas na Tabela 10.5: 
TABELA 10.5 - Propriedades elásticas globais do meio compósito da Figura 10.4.

\begin{tabular}{cccccc}
\hline \multirow{2}{*}{ Material } & \multicolumn{5}{c}{ Propriedades elásticas (direções globais) } \\
\cline { 2 - 6 } & $E_{x}(\mathrm{MPa})$ & $E_{y}(\mathrm{MPa})$ & $v_{x y}$ & $v_{y x}$ & $G_{x y}(M P a)$ \\
\hline $\begin{array}{c}\text { Chapa } \\
\text { compósita }\end{array}$ & $31.378,22$ & $25.418,03$ & 0,16 & 0,13 & $13.525,68$ \\
\hline
\end{tabular}

Salienta-se que, utilizando-se as propriedades elásticas contidas na Tabela 10.5, ou na Tabela 10.3 da primeira aplicação, pode-se simular um meio considerado homogêneo e se obter as mesmas soluções de deformações (nas duas direções da estrutura) alcançadas com as duas simulações anteriores. Essa análise também foi realizada com o código implementado e os resultados encontrados foram os esperados.

\subsubsection{Chapa com anisotropia geral - fibras dispostas em duas direções ortogonais e não coincidentes com as direções globais do problema}

A chapa analisada está apresentada na Figura 10.5:

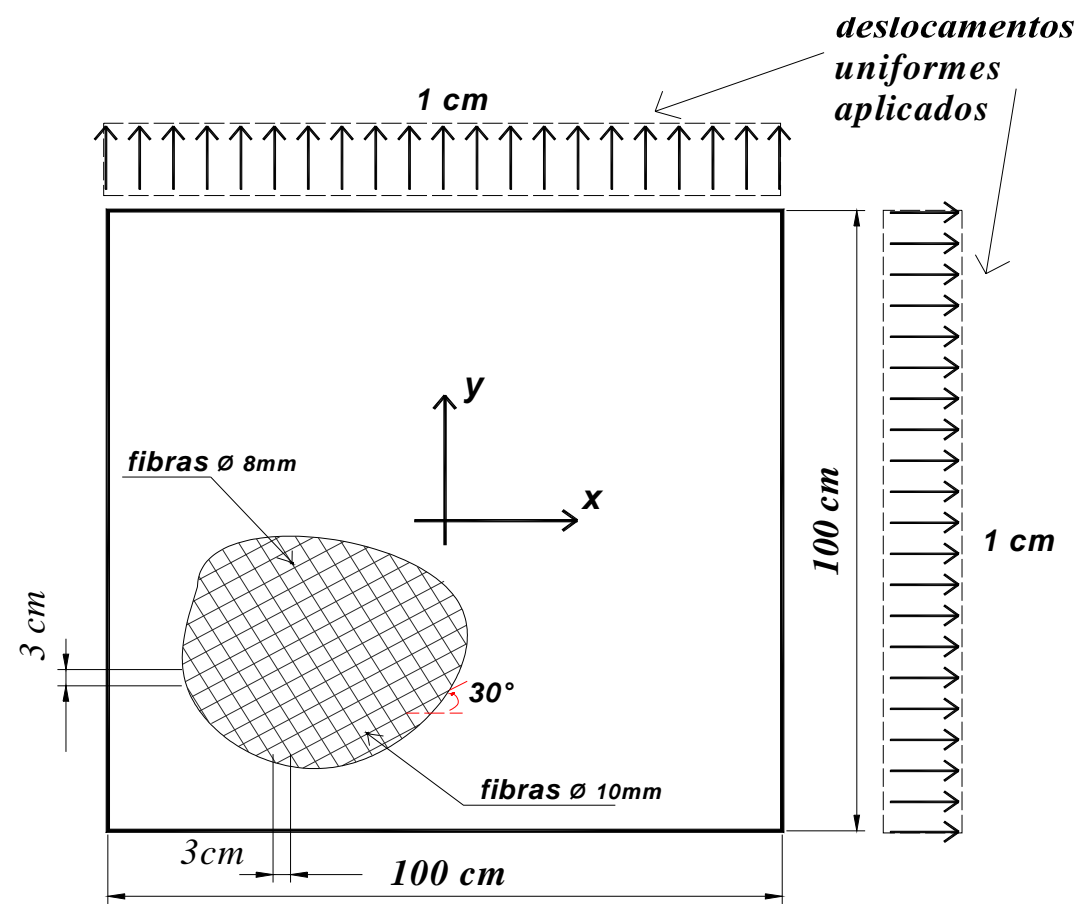

FIGURA 10.5 - Chapa reforçada em duas direções não coincidentes com os eixos globais da estrutura.

Esta aplicação difere da anterior pelo fato da armação quadriculada, formada pelas duas linhas perpendiculares de fibras, estar posicionada de maneira não 
coincidente com os eixos globais do compósito (eixos $x$ e $y$ ), fazendo-se com que, na aplicação de uma solicitação em uma das direções globais, a chapa tenha um comportamento fortemente anisotrópico onde surgirão, inclusive, deformações tangenciais num problema de tração simples devido a presença dos coeficientes de influência mútua no tensor constitutivo do material.

Assim, utilizando-se o programa desenvolvido, pode-se simular esse tipo de estrutura e se obter propriedades elásticas de materiais com anisotropia geral. Com isso, ao se aplicar deslocamentos nas direções globais da chapa da Figura 10.5, comentandose que isoladamente os materiais empregados possuem as características contidas na Tabela 10.4, encontra-se as propriedades elásticas relacionadas nas Tabela 10.6 e 10.7:

TABELA 10.6 - Propriedades elásticas de resistência obtidas para a chapa anisotrópica da Figura 10.5.

\begin{tabular}{cccc}
\hline \multirow{2}{*}{ Material } & \multicolumn{4}{c}{ Propriedades Elásticas de Resistência (direções globais) } \\
\cline { 2 - 4 } & $E_{x}(\mathrm{MPa})$ & $E_{y}(\mathrm{MPa})$ & $G_{x y}(\mathrm{MPa})$ \\
\hline $\begin{array}{c}\text { Chapa } \\
\text { Compósita }\end{array}$ & $24.855,65$ & $22.648,91$ & $9.465,21$ \\
\hline
\end{tabular}

TABELA 10.7 - Coeficientes elásticos obtidos para a chapa anisotrópica da Figura 10.5.

\begin{tabular}{ccccc}
\hline & \multicolumn{4}{c}{ Coeficientes Elásticos (direções globais) } \\
\cline { 2 - 5 } Material & $\eta_{x y, x}$ & $\eta_{x y, y}$ & $v_{x y}$ & $v_{y x}$ \\
\hline $\begin{array}{c}\text { Chapa } \\
\text { Compósita }\end{array}$ & $-0,309$ & $-0,128$ & 0,313 & 0,285 \\
\hline
\end{tabular}

Para obtenção dos coeficientes de influência mútua que, como exposto no capítulo 3, são específicos para materiais anisotrópicos gerais e exprimem o relacionamento entre deformações tangenciais e normais, é preciso medir as distorções presentes na chapa após a aplicação da solicitação. Para o presente exemplo, tem-se a seguinte configuração de deformação (desenho sem escala) quando da aplicação do deslocamento unitário na direção $x$ (Figura 10.6): 


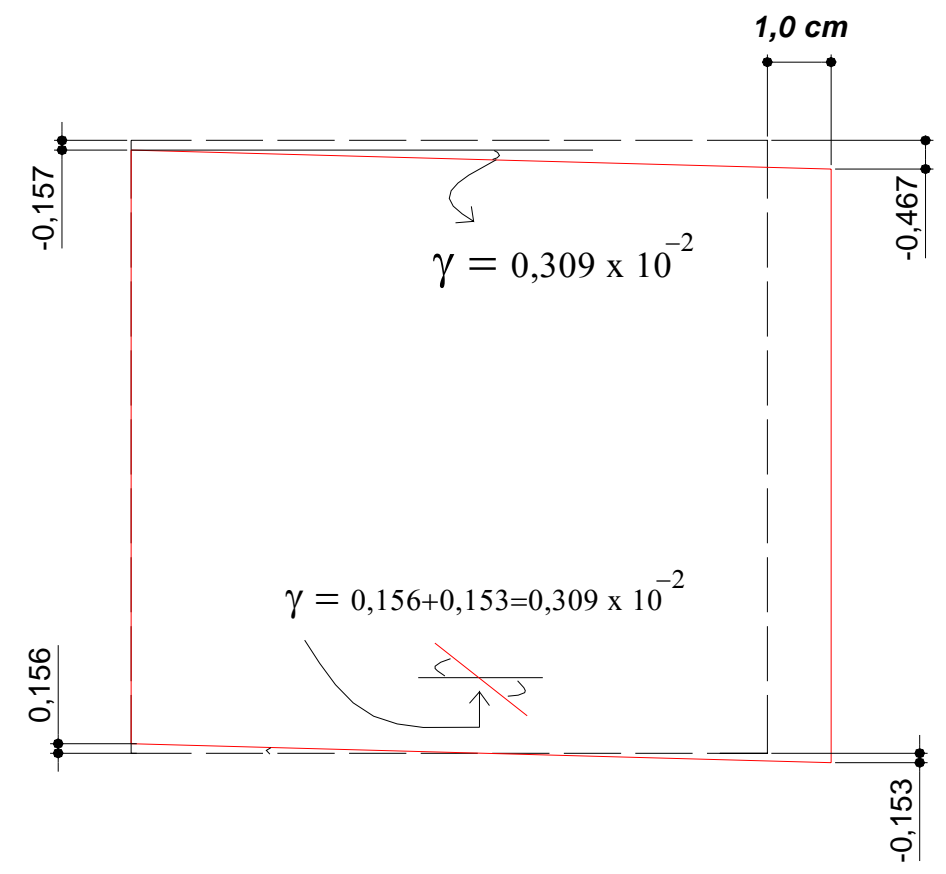

FIGURA 10.6 - Configuração deformada, em vermelho, da chapa da Figura 10.5 quando o deslocamento é aplicado em $x$.

Na aplicação do mesmo deslocamento na direção $y$, tem-se:

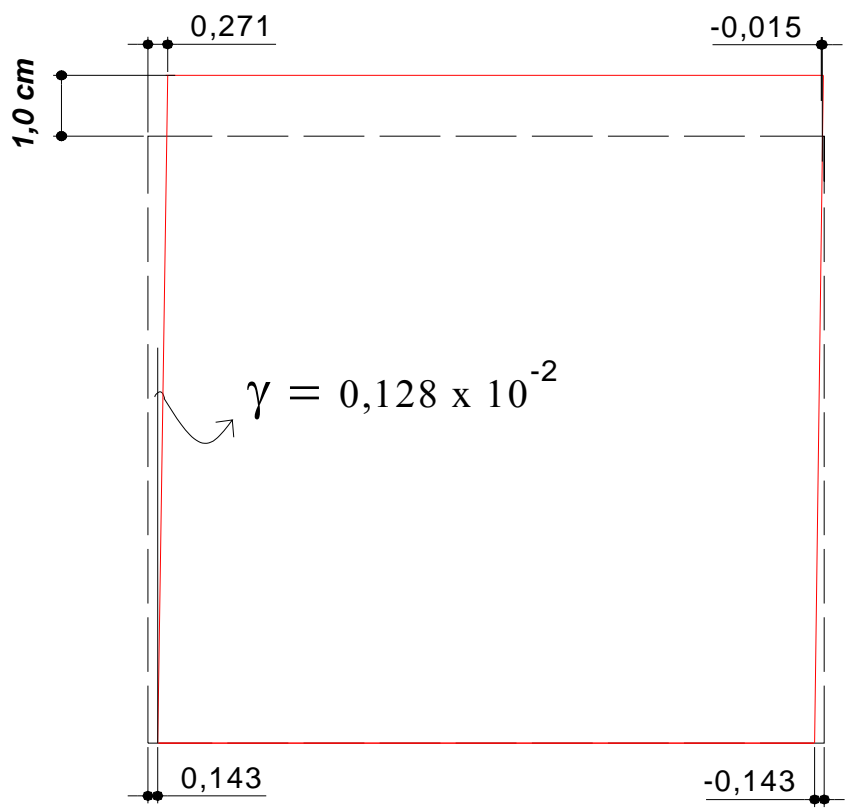

FIGURA 10.7 - Configuração deformada, em vermelho, da chapa da Figura 10.5 quando o deslocamento é aplicado em $y$.

Podendo-se então, extrair as seguintes relações: 


$$
\begin{aligned}
& \eta_{x y, x}=\frac{\gamma_{x y}}{\varepsilon_{x}}=\frac{0,309 \times 10^{-2}}{1,0 \times 10^{-2}}=0,309 \Rightarrow-0,309 \\
& \eta_{x y, y}=\frac{\gamma_{x y}}{\varepsilon_{y}}=\frac{0,128 \times 10^{-2}}{1,0 \times 10^{-2}}=0,128 \Rightarrow-0,128
\end{aligned}
$$

Os sinais negativos para os coeficientes de influência mútua são assumidos devido ao fato da distorção ocorrida ter causado um encurtamento na direção ortogonal à aplicação do deslocamento.

Com os dados das Tabelas 10.6 e 10.7, pode-se simular novamente o mesmo exemplo, porém, considerando a chapa homogênea. Foram obtidos, como esperado, os mesmo resultados de tensões e de deformações.

\subsubsection{Viga de Concreto Armado}

Neste exemplo, simula-se uma viga de concreto, normalmente armada, mostrada na Figura 10.8:
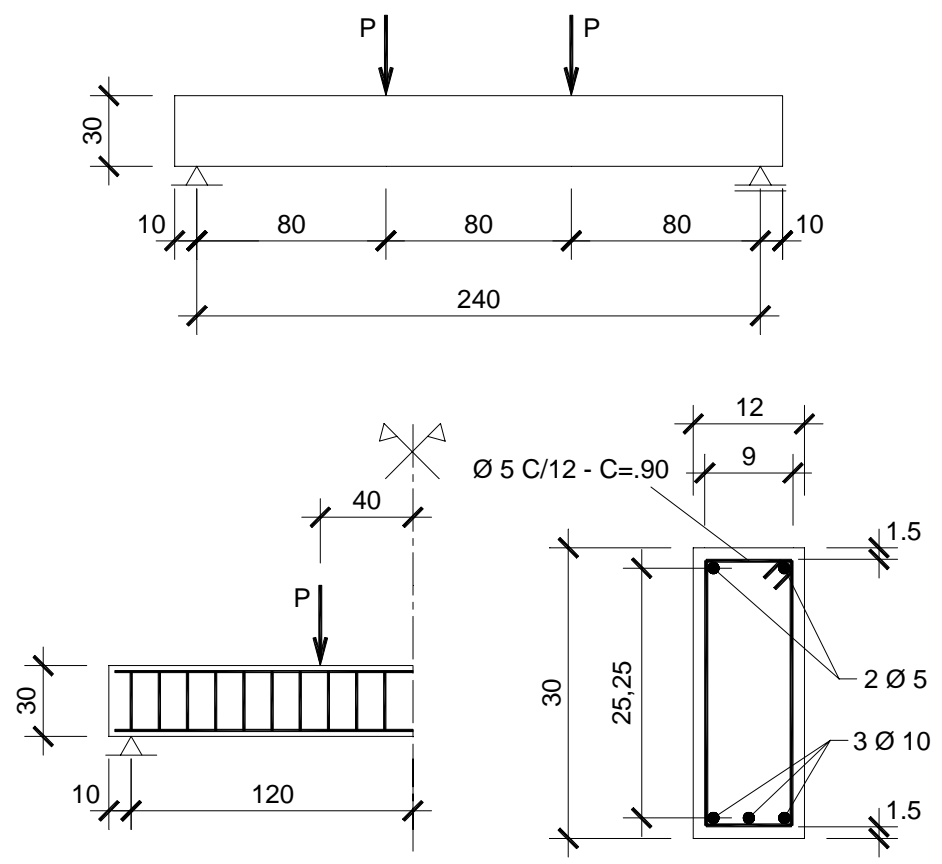

FIGURA 10.8 - Viga de concreto normalmente armada. Características gerais. 
Os resultados obtidos são comparados com resultados experimentais obtidos por TAKEYA (1972) e também com os resultados numéricos (MEC) apresentados por CODA (2001).

Para tanto, adota-se para os materiais (concreto e aço) as seguintes propriedades elásticas, Tabela 10.8:

TABELA 10.8 - Propriedades dos materiais que constituem a viga de concreto analisada.

\begin{tabular}{ccccc}
\hline \multirow{2}{*}{ Material } & \multicolumn{4}{c}{ Propriedades elásticas (direções globais) } \\
\cline { 2 - 5 } & $E_{x}(M P a)$ & $E_{y}(M P a)$ & $v$ & $G_{x y}(M P a)$ \\
\hline Aço & $196.000,0$ & $196.000,0$ & 0,3 & $80.000,0$ \\
\hline Matriz cimentícia & $13.500,0$ & $13.500,0$ & 0,2 & $5.700,0$ \\
\hline
\end{tabular}

Para a análise não linear, são utilizados, para comparação, duas superfícies de escoamento, a de TSAI \& WU (1971) e a de Drucker-Praguer. Assume-se comportamento plástico perfeito para ambos materiais. São adotadas as seguintes tensões de escoamento:

TABELA 10.9 - Tensões de escoamento para os materiais empregados.

\begin{tabular}{ccc}
\hline \multirow{2}{*}{ Tensões } & \multicolumn{3}{c}{ Materiais } \\
\cline { 2 - 3 } & Concreto $(\mathrm{MPa})$ & Aço $(\mathrm{MPa})$ \\
\hline $\operatorname{Tração}\left(f_{t}\right)$ & 1,20 & 500,0 \\
\hline Compressão $\left(f_{c}\right)$ & 27,3 & 500,0 \\
\hline
\end{tabular}

Para a modelagem numérica da viga de concreto (Figura 10.8), são empregados 20 x 20 elementos finitos (horizontal $x$ vertical) QST, atribuindo-se assim, 1922 graus de liberdade para a análise da matriz. As fibras, como comentado, são modeladas como elementos finitos de barra simples, com dois graus de liberdade por nó. Foram empregados 32 elementos para as fibras. São utilizados 200 incrementos de carga com uma precisão de $10^{-6}$ para tensões e deslocamentos.

De posse de todas essas informações, pôde-se então realizar a simulação e se obter os resultados mostrados na Figura 10.9: 


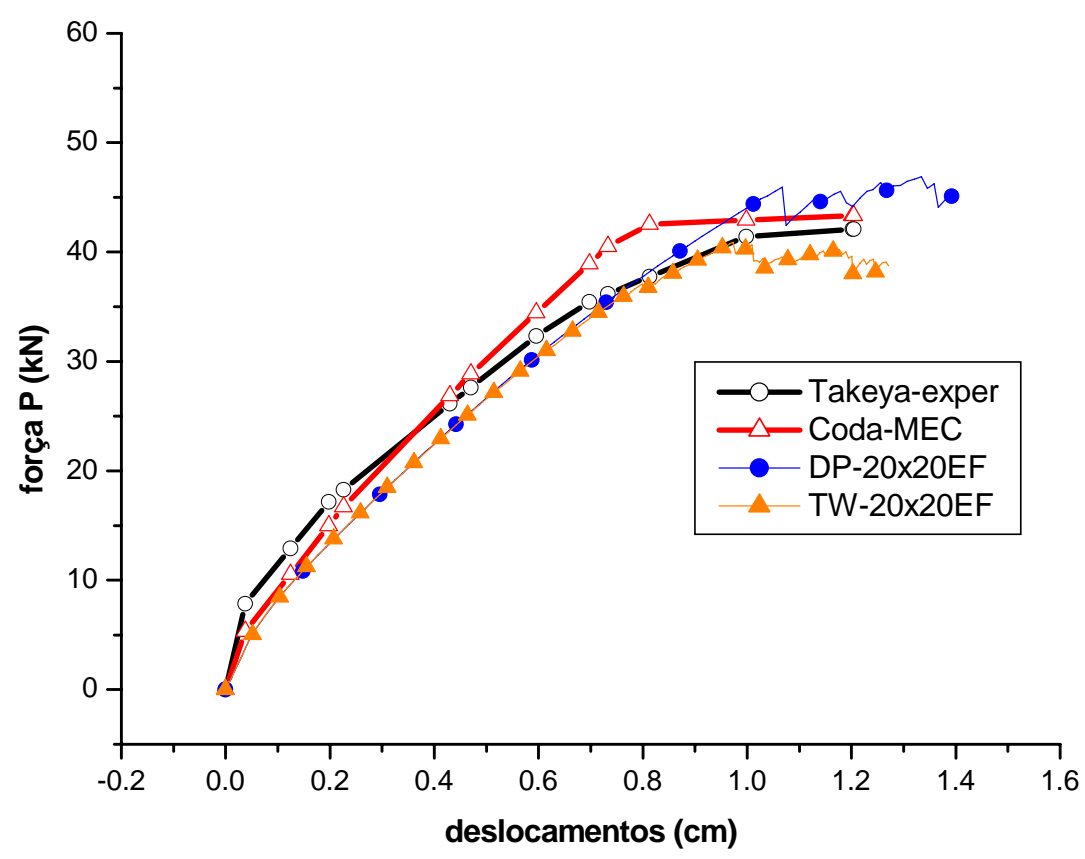

FIGURA 10.9 - Relação força $\mathbf{P}$ versus deslocamentos do vão central da viga de concreto.

No gráfico, observa-se que os dois resultados numéricos obtidos estão muito próximos dos fornecidos por TAKEYA (1972) e por CODA (2001), sendo que, a solução oriunda da utilização do critério de TSAI \& WU (1971) se mostra mais suave e próxima da resposta experimental. A diferença entre as respostas advindas da utilização dos dois critérios justifica-se pelo fato de suas superfícies possuírem termos diferentes relacionados com a tensão de escoamento de tração.

\subsubsection{Viga de Concreto Reforçado com Fibras de Aço - Ensaios de Furlan Jr. (1995)}

Neste exemplo, simula-se quatro vigas de concreto reforçadas com fibras de aço, sem a presença de armaduras transversais, que foram ensaiadas por FURLAN Jr. (1995). A geometria padrão das vigas está apresentada na Figura 10.10: 


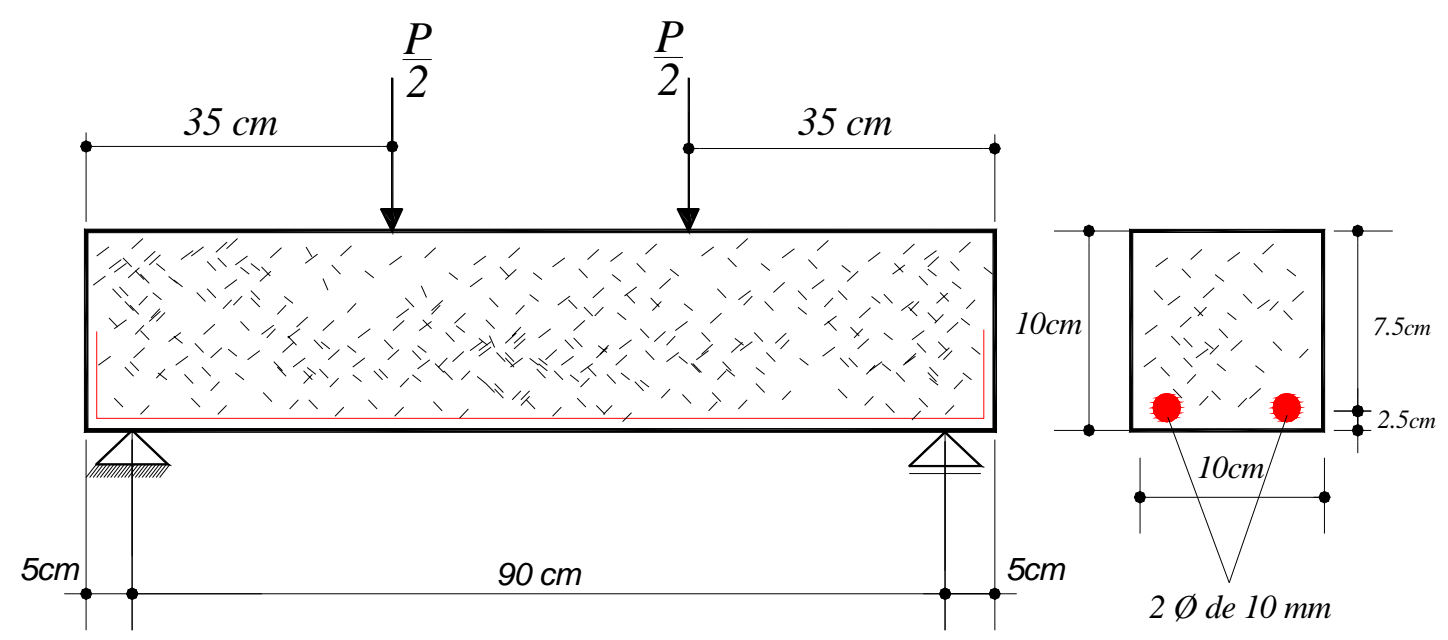

FIGURA 10.10 - Viga de concreto reforçada com fibras. Características gerais.

Com a apresentação desses exemplos, visa-se demonstrar a aplicabilidade do código desenvolvido para as análises que envolvam reforços de fibras curtas, sejam elas, de aço, sintéticas ou vegetais. Os resultados estão organizados em diagramas força versus deslocamento do vão central das vigas analisadas. São utilizados, como no exemplo anterior, as superfícies de escoamento de TSAI \& WU (1971) e DruckerPraguer podendo-se, dessa maneira, ter duas opções para a análise de resistência.

Tratando-se dos materiais empregados (concreto, barras e fibras de aço) têm-se as seguintes propriedades elásticas, Tabela 10.10:

TABELA 10.10 - Propriedades dos materiais que constituem as vigas analisadas.

\begin{tabular}{ccccc}
\hline \multirow{2}{*}{ Material } & \multicolumn{5}{c}{ Propriedades elásticas (direções globais) } \\
\cline { 2 - 5 } & $E_{x}(\mathrm{MPa})$ & $E_{y}(\mathrm{MPa})$ & $v$ & $G_{x y}(\mathrm{MPa})$ \\
\hline Aço & $196.000,0$ & $196.000,0$ & 0,3 & $80.000,0$ \\
\hline $\begin{array}{c}\text { Fibras curtas de } \\
\text { aço }\end{array}$ & $196.000,0$ & $196.000,0$ & 0,3 & $80.000,0$ \\
\hline Matriz cimentícia & $17.500,0$ & $17.500,0$ & 0,2 & $7.000,0$ \\
\hline
\end{tabular}

Na análise não linear, assume-se novamente comportamento plástico perfeito para os materiais. São adotadas as seguintes tensões de escoamento, baseando-se nas curvas experimentais de caracterização fornecidas por FURLAN Jr. (1995): 
TABELA 10.11 - Tensões de escoamento para os materiais empregados.

\begin{tabular}{cccc}
\hline \multirow{2}{*}{ Tensões } & \multicolumn{3}{c}{ Materiais } \\
\cline { 2 - 4 } & Concreto $(\mathrm{MPa})$ & Aço $(\mathrm{MPa})$ & Fibras de aço $(\mathrm{MPa})$ \\
\hline Tração $\left(f_{t}\right)$ & 2,0 & 500,0 & $1.100,0$ \\
\hline $\begin{array}{c}\text { Compressão } \\
\left(f_{c}\right)\end{array}$ & 40,0 & 500,0 & $1.100,0$ \\
\hline
\end{tabular}

As quatro vigas simuladas se diferenciam em relação às fibras de aço da mistura de concreto, especificamente, pelo tamanho da fibra e respectivo volume de fibras da mistura (FURLAN Jr, 1995). Desta maneira é preciso mostrar a quantidade de fibras empregadas em cada análise, separando-se as vigas da seguinte maneira:

TABELA 10.12 - Características e quantidade das fibras utilizadas em cada viga simulada.

\begin{tabular}{ccccc}
\hline Vigas & $\begin{array}{c}\text { Comprimento } \\
\text { da fibra }\end{array}$ & $\begin{array}{c}\text { Volume }\left(\mathrm{cm}^{3}\right) \\
\text { de uma fibra }\end{array}$ & $\begin{array}{c}\text { \% de fibras em } \\
\text { volume da viga }\end{array}$ & $\begin{array}{c}\text { Quantidade de } \\
\text { fibras na mistura }\end{array}$ \\
\hline 1 & 1 " $-2,54 \mathrm{~cm}$ & 0,011684 & $1 \%$ & 8.558 \\
\hline 2 & $1 ”-2,54 \mathrm{~cm}$ & 0,011684 & $2 \%$ & 17.117 \\
\hline 3 & $1 ” 1 / 2-3,81 \mathrm{~cm}$ & 0,017526 & $1 \%$ & 5.705 \\
\hline 4 & $1 ” 1 / 2-3,81 \mathrm{~cm}$ & 0,017526 & $2 \%$ & 11.410
\end{tabular}

Obs.: área da seção transversal das fibras: 0,0046 $\mathrm{cm}^{2}$; Volume da viga de concreto: $10.000 \mathrm{~cm}^{3}$

É importante observar que nos ensaios realizados por FURLAN Jr. (1995), as fibras estavam dispersas de forma tridimensional e aleatória e, no código computacional para análise plana aqui desenvolvido, as fibras são distribuídas randomicamente no plano de ação do carregamento. Com isso, visando-se dar consistência à simulação numérica feita, considerou-se as vigas com largura unitária (hipótese que permite uma análise plana da viga), dividindo-se também, a quantidade de fibras (indicada na Tabela 10.12) por 10. Salienta-se que a solução numérica do problema (força ou deslocamento, dependendo-se de qual solicitação foi aplicada) deve ser multiplicada por 10 para se obter resultados compatíveis com os experimentais.

Para a definição da discretização adequada de elementos finitos de chapa na modelagem numérica, foi realizado um breve estudo de convergência podendo-se assim, se definir com mais clareza alguns parâmetros, como, número de elementos e tolerância 
da análise não-linear. Os gráficos construídos para esse fim, para os dois critérios, são mostrados nas Figura 10.11 a 10.14:

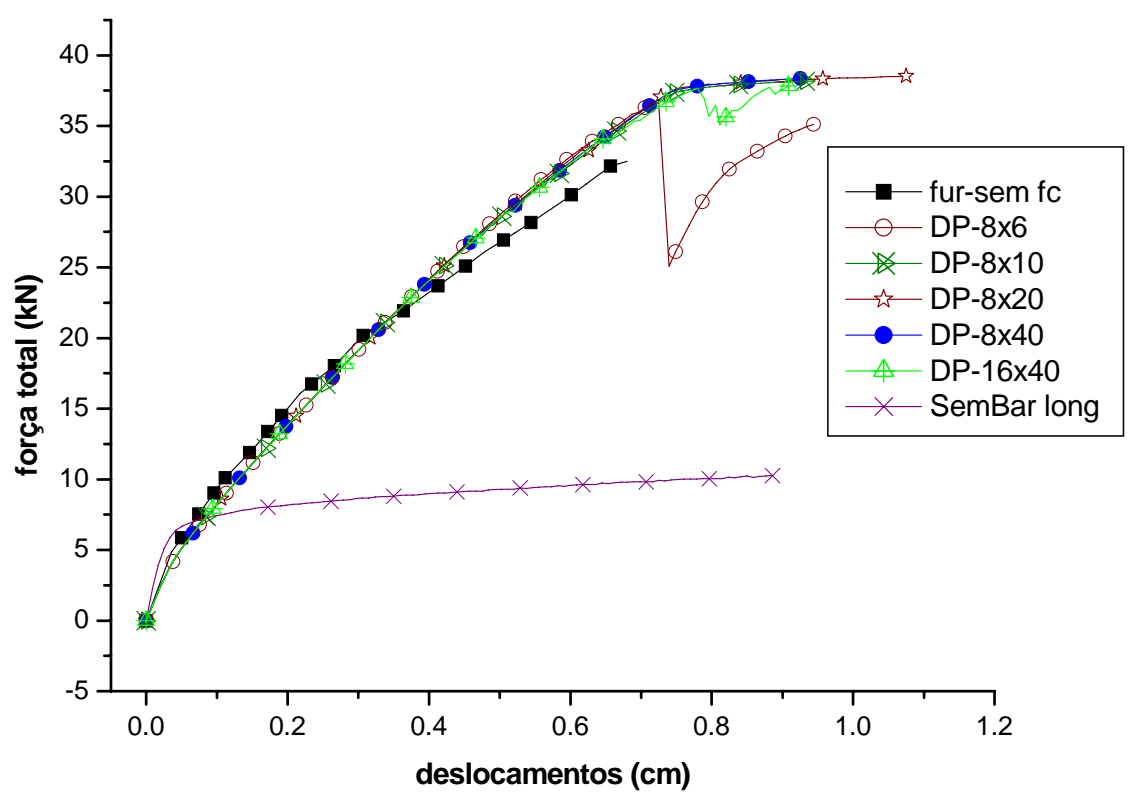

FIGURA 10.11 - Soluções para diferentes malhas. Critério de Drucker-Praguer.

No gráfico anterior, a curva na cor preta, é a curva média do ensaio experimental de FURLAN Jr. (1995) para a viga da Figura 10.10 sem a adição de fibras curtas na mistura de concreto. Mostra-se ainda na Figura 10.11 soluções numéricas para diferentes discretizações de elementos finitos QST, considerando-se a viga apenas reforçada com as barras longitudinais. Uma solução numérica para a viga sem esses dois tipos de barras (longitudinal e fibras curtas) é também apresentada.

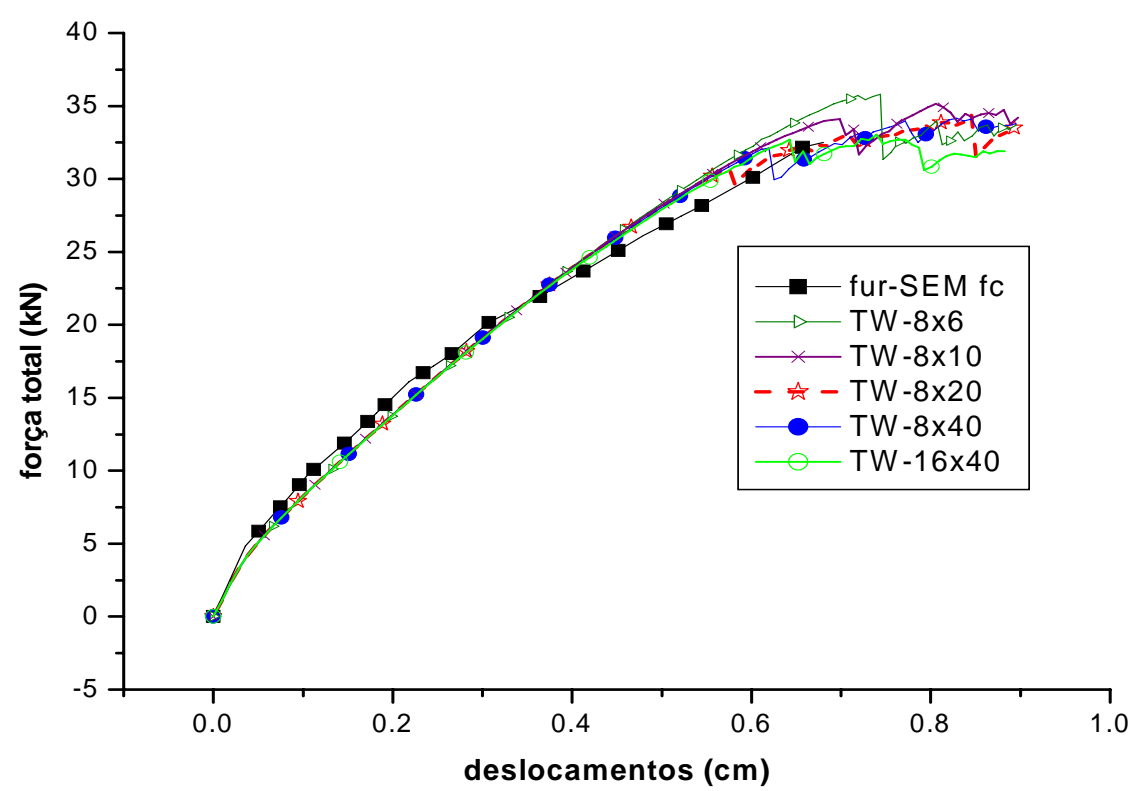

FIGURA 10.12 - Soluções para diferentes malhas. Critério de TSAI \& WU (1971). 
É importante dizer que devido a condição de simetria do problema, somente metade da estrutura foi discretizada e assim, nas legendas dos gráficos anteriores, o primeiro número das malhas se refere ao número de elementos que discretizam metade do vão da viga. Para ambos critérios, a malha de 8 x 20 elementos se mostrou adequada sem exigir um custo computacional elevado, por isso, é a malha empregada nas análises.

Para análise da tolerância de tensões e de deslocamento a ser empregada, têmse os gráficos a seguir:

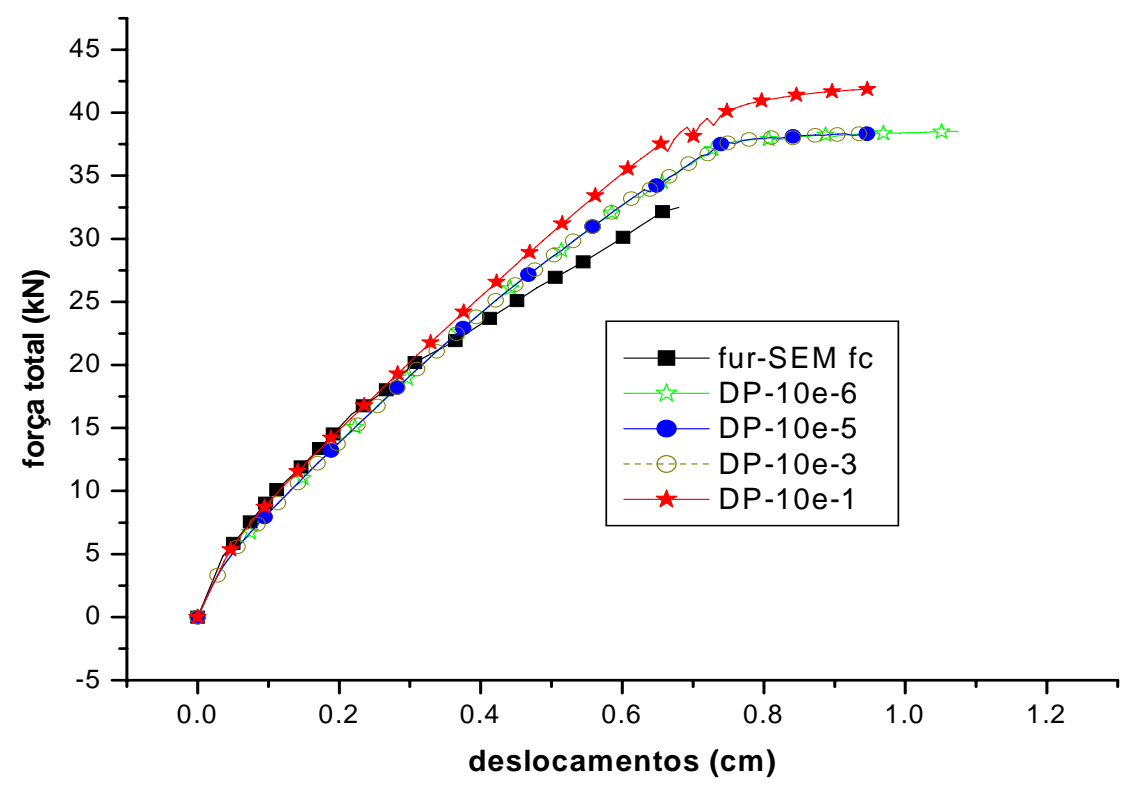

FIGURA 10.13 - Soluções para diferentes tolerâncias. Critério de Drucker-Praguer.

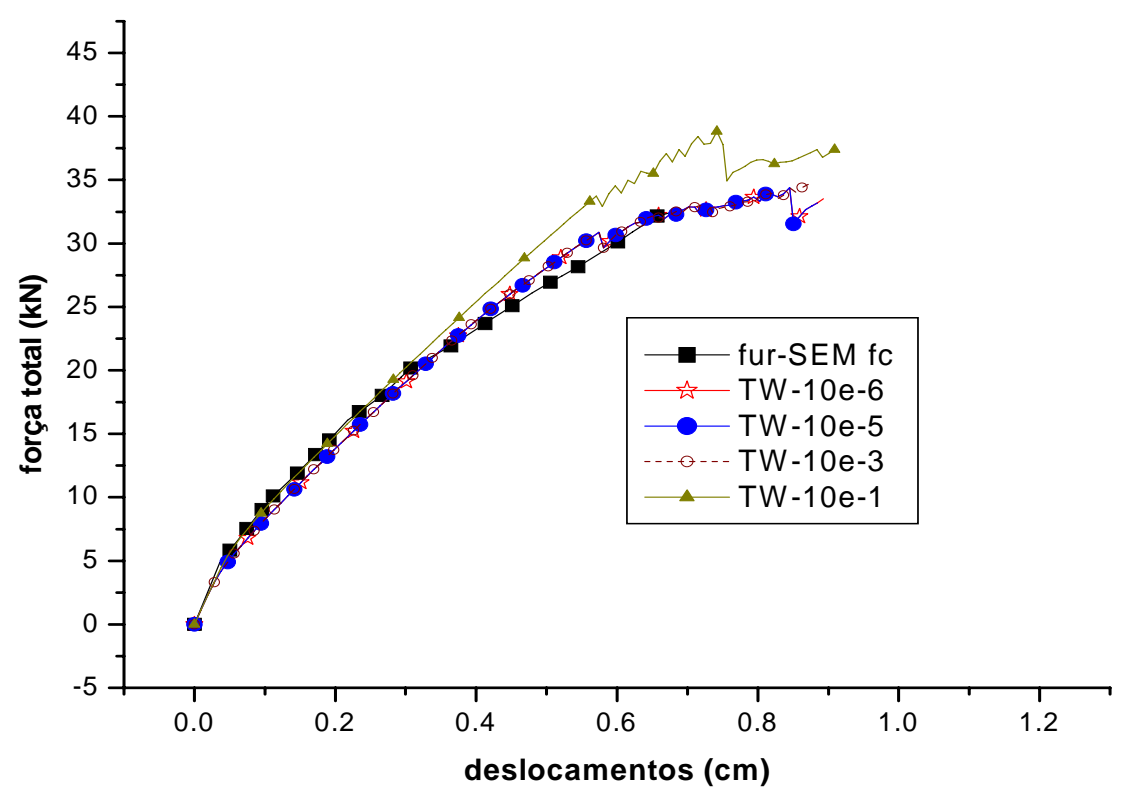

FIGURA 10.14 - Soluções para diferentes tolerâncias. Critério de TSAI \& WU (1971). 
Nas soluções apresentadas, pode-se observar que a tolerância de $10^{-5}$ é adequada para os critérios e assim, pode-se utiliza-la na continuidade das análises. Esta tolerância foi empregada também nos testes de convergência de malha.

De posse de todas essas informações, pôde-se então realizar a simulação das quatro vigas reforçadas por fibras e se obter os resultados mostrados a seguir.

Para a viga 1 (Tabela 10.12) tem-se:

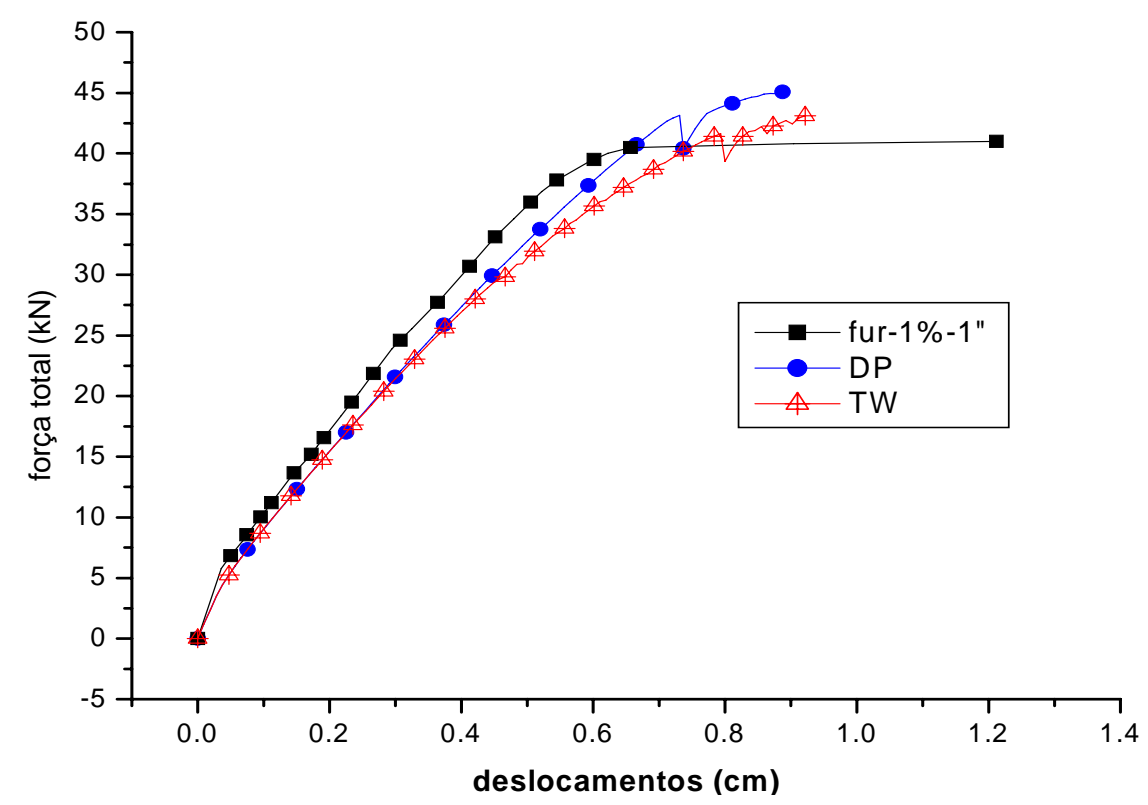

FIGURA 10.15 - Relação força $x$ deslocamento no meio do vão da viga 1.

Para a viga 2: 


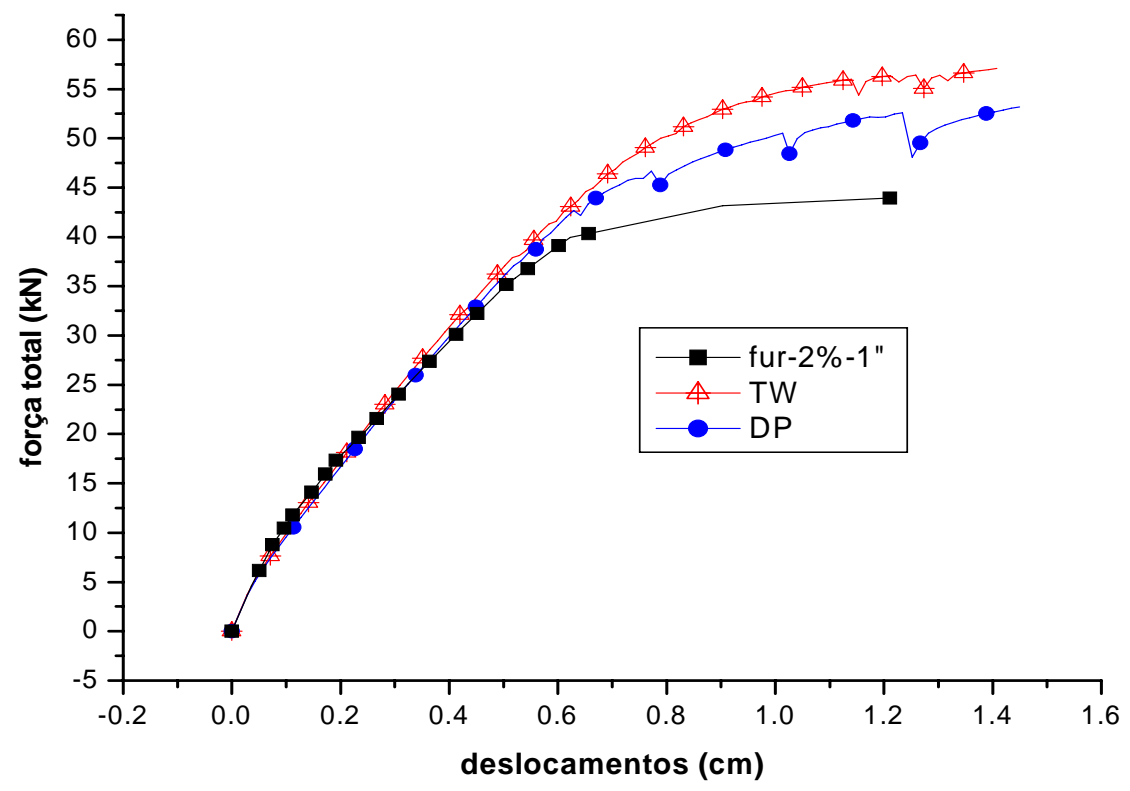

FIGURA 10.16 - Relação força $x$ deslocamento no meio do vão da viga 2.

Para a viga 3:

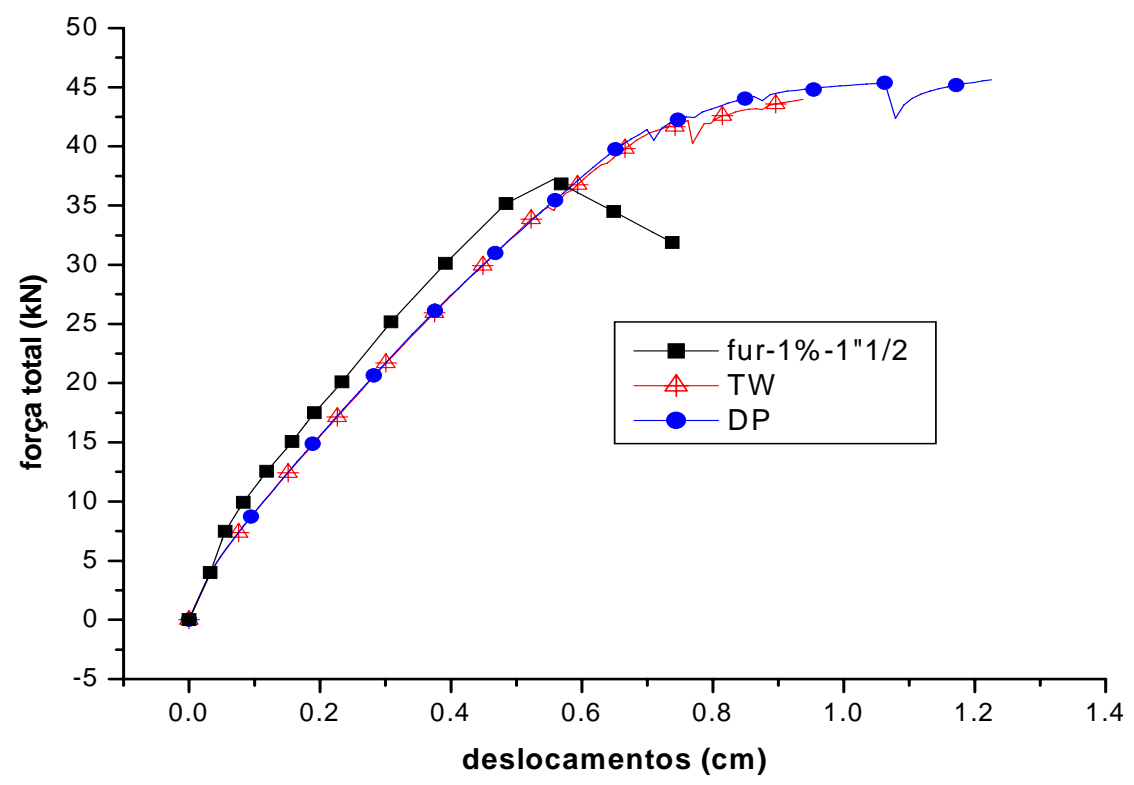

FIGURA 10.17 - Relação força $x$ deslocamento no meio do vão da viga 3.

Para a viga 4: 


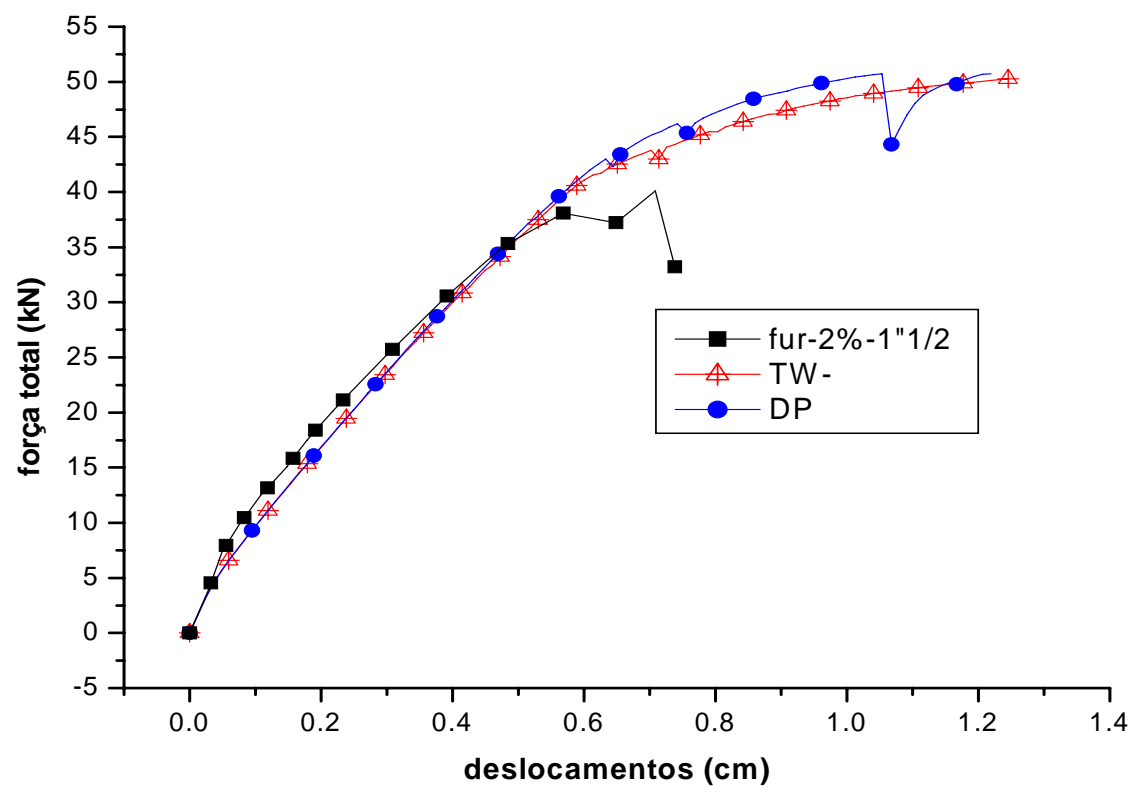

FIGURA 10.18 - Relação força $x$ deslocamento no meio do vão da viga 4.

Nos resultados apresentados, é possível observar um enrijecimento presente nas vigas devido a presença do reforço com fibras curtas, quando os resultados são confrontados com os mostrados nas Figuras 11 a 14. Quando comparados com os resultados experimentais, percebe-se também que as soluções numéricas estão mais rígidas, principalmente para as vigas 3 e 4 . No entanto, as soluções para as vigas 1 e 2 são mais próximas da curva experimental.

Essas diferenças são perfeitamente explicáveis pelo fato dos resultados experimentais estarem cercados de fatores que nem sempre são equacionáveis. Salientase ainda que a formulação implementada até o presente momento não considera o deslizamento das armaduras longitudinais e nem das fibras, e assim, uma rigidez maior das soluções é realmente possível.

Um outro fator a se considerar é sobre a aleatoriedade do posicionamento das fibras curtas. No ensaio experimental (tridimensional) as fibras podem ter se posicionados de forma a não contribuírem efetivamente para a rigidez da estrutura. $\mathrm{Na}$ simulação numérica, a rigidez de cada fibra é sempre considerada no sistema aumentado-se, naturalmente, a rigidez da viga.

Com o intuito de se investigar melhor a participação das fibras na rigidez dos problemas em questão, apresenta-se a seguir mais algumas soluções obtidas considerando-se desta vez somente a presença de $70 \%$ da quantidade de fibras dos exemplos anteriores, retirando-se assim, de forma arbitrária aproximadamente $30 \%$ das 
fibras (fibras essas que podem não estar contribuindo efetivamente para a resistência do corpo de prova - hipótese adotada).

Para a viga 1, tem-se:

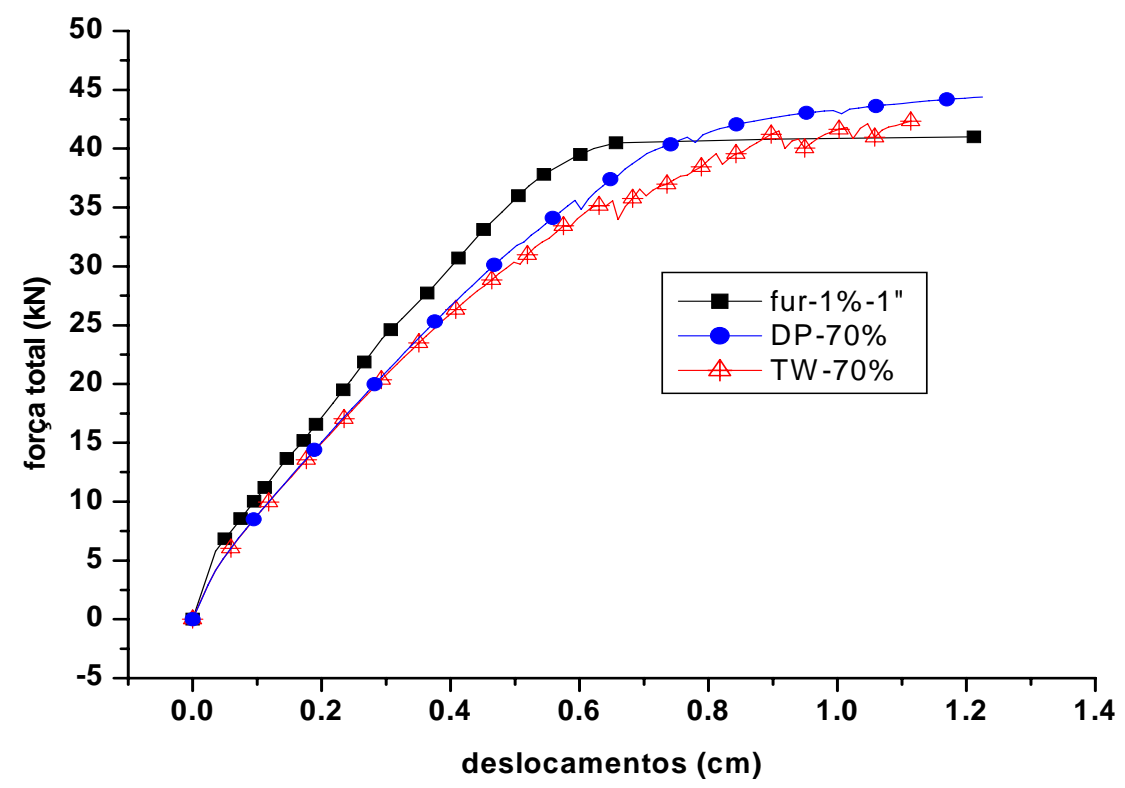

FIGURA 10.19 - Relação força $x$ deslocamento no meio do vão da viga $1.70 \%$ de fibras

Para a viga 2:

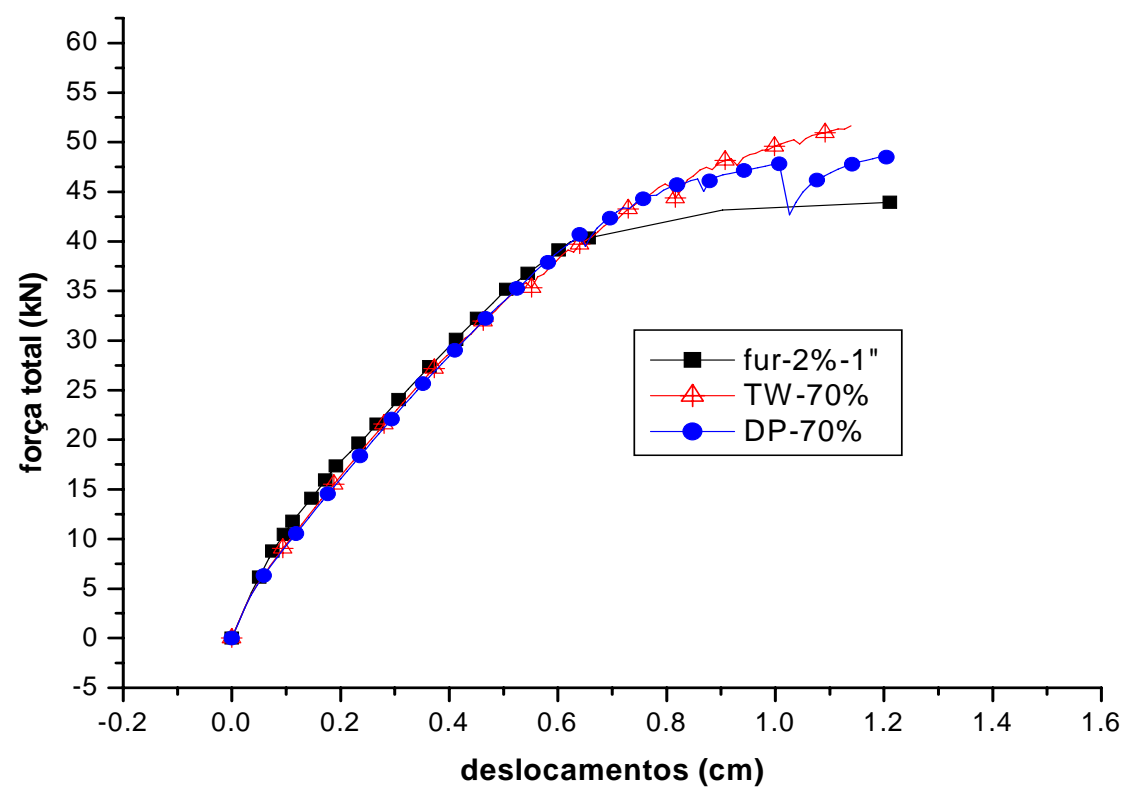

FIGURA 10.20 - Relação força $x$ deslocamento no meio do vão da viga 2. 70\% de fibras 
Para a viga 3:

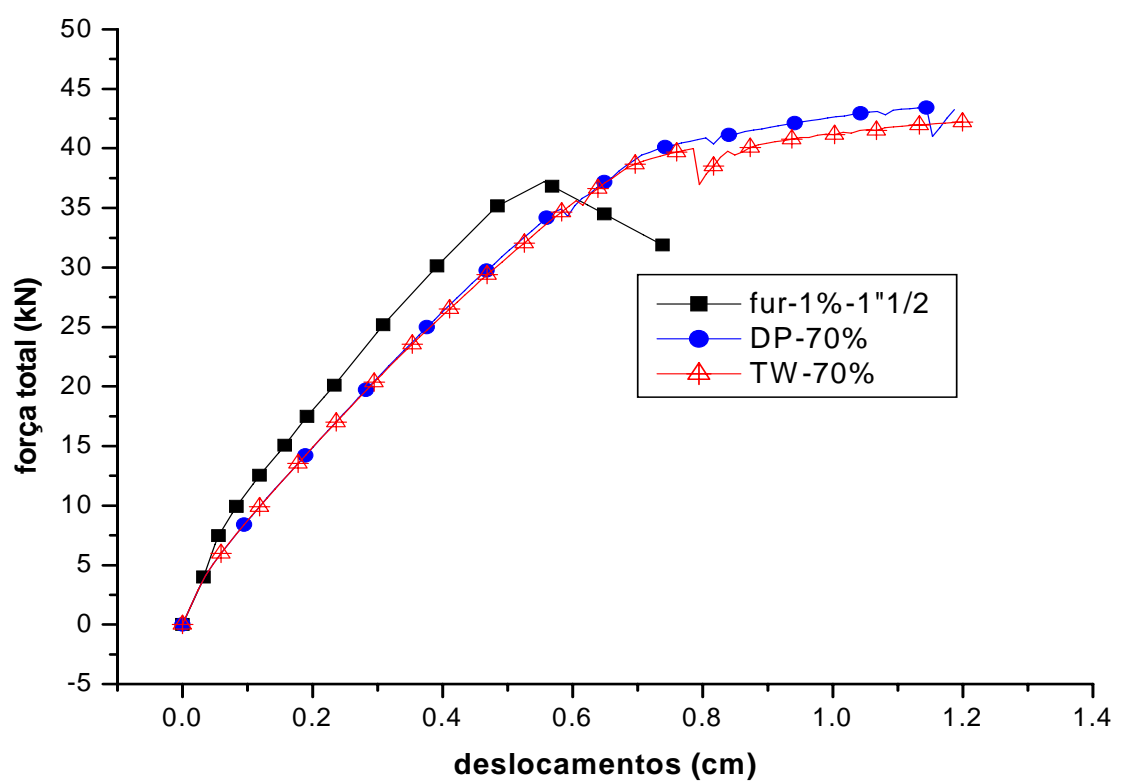

FIGURA 10.21 - Relação força $x$ deslocamento no meio do vão da viga 3. 70\% de fibras

Para a viga 4:

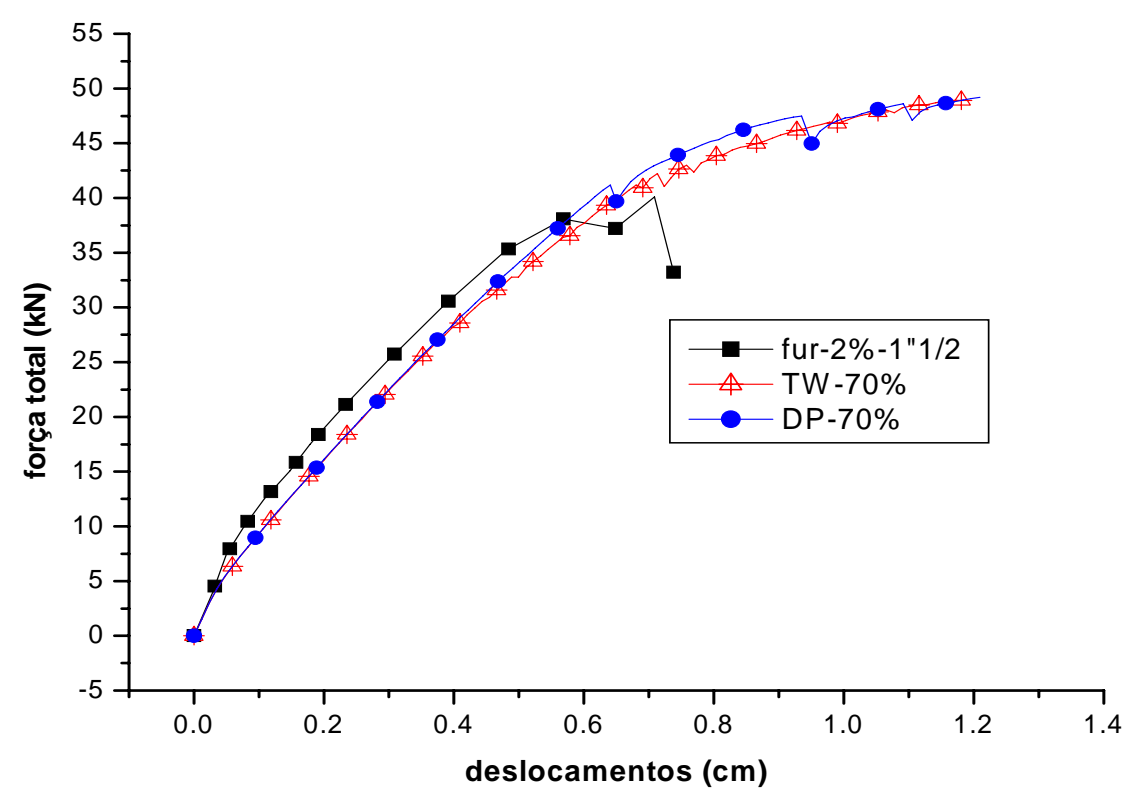

FIGURA 10.22 - Relação força $x$ deslocamento no meio do vão da viga 4. 70\% de fibras 
Com essa nova hipótese, observa-se uma concordância maior entre as soluções numéricas e experimentais, levando à conclusão que o desenvolvimento do software 3D seria adequado para este tipo de análise.

Para finalizar este capítulo, é importante ainda comentar, que a formulação desenvolvida, bem como, o código computacional implementado, são contribuições originais na área de pesquisa em meios anisotrópicos e que ambas ferramentas de análise podem ser enriquecidas com contribuições futuras de outros pesquisadores, do mesmo departamento da EESC ou de outras instituições.

No capítulo seguinte, as conclusões da presente pesquisa são apresentadas. 


\section{CONCLUSÃO}

Neste último capítulo, são apresentadas as conclusões da presente tese de doutoramento e algumas discussões pertinentes aos temas abordados. São feitas sugestões para a continuação da pesquisa visando mostrar que o presente trabalho pode servir de base para outras pesquisas relacionadas à modelagem numérica de meios anisotrópicos. Considera-se que o programa desenvolvido pode ser aplicado em trabalhos experimentais de investigação de forma a se estabelecer parâmetros para futuros desenvolvimentos.

\subsection{CONSIDERAÇÕES CONCLUSIVAS GERAIS}

O principal objetivo do trabalho foi o desenvolvimento de formulações e de dois códigos computacionais (um baseado no MEF e o outro no MEC) que permitissem a análise bidimensional estática de meios anisotrópicos, reforçados ou não por fibras, com não linearidade física (plasticidade e viscoplasticidade).

É importante neste momento evidenciar mais uma vez o motivo pelo qual se decidiu implementar dois programas semelhantes, com duas técnicas numéricas diferentes, para o tratamento de problemas anisotrópicos. Inicialmente, na concepção do projeto de pesquisa, já se conhecia a dificuldade de se encontrar na literatura resultados relacionados a problemas não lineares em meios anisotrópicos, principalmente, com anisotropia geral, resultados esses que seriam importantes para aferição das soluções numéricas oriundas da pesquisa que se pretendia realizar. Com relação ao MEC não linear, já era de conhecimento também que, comparando-se com o MEF, suas formulações anisotrópicas e as conseqüentes implementações são muito mais complexas 
e inexistentes. Baseando-se nesses fatos, decidiu-se então por desenvolver primeiramente o código para análise linear de meios anisotrópicos com o MEF e depois, o programa pelo MEC, podendo-se assim, se comparar e aferir as soluções obtidas com ambas formulações. Depois, todas as demais implementações que foram realizadas seguiram a mesma metodologia numérica, garantindo-se com isso, ao menos uma certeza da correção das implementações. Essa estratégia foi fundamental para se ter confiança nos códigos implementados.

Sobre a originalidade da presente pesquisa de doutorado, deve-se comentar que ela já seria garantida apenas pelo fato do trabalho ter como tema o tratamento de plasticidade, pelo MEC, em meios com anisotropia geral utilizando-se a solução fundamental de CRUSE \& SWEDLOW (1971), ainda não empregada para a análise de problemas que possuem essa abordagem constitutiva. No entanto, a pesquisa também tratou de outros assuntos inéditos na área de modelagem numérica de corpos anisotrópicos:

- Análise viscoelástica e viscoplástica pelo MEC;

- Análise viscoplástica diferencial pelo MEF;

- Determinação de um algoritmo de retorno com uma expressão fechada do multiplicador plástico para a superfície de TSAI \& WU (1971);

- Modelagem de fibras imersas em posição qualquer do domínio modelado pelo MEF sem aumento de graus de liberdade.

Assim, pode-se observar que os temas relacionados conferem ao trabalho uma originalidade maior do que o previsto no início da pesquisa, garantindo-se contribuições científicas na área de conhecimento na qual ele está inserido.

$\mathrm{Na}$ seção seguinte, conclusões e considerações pertinentes às soluções numéricas, para as aplicações feitas, obtidas com os dois programas são detalhadas visando-se, dessa maneira, evidenciar ainda melhor os comentários conclusivos já realizados nos capítulos 7 a 10 . 


\subsection{CONCLUSÕES RELACIONADAS COM AS APLICAÇÕES}

Nas aplicações dos códigos desenvolvidos, em problemas elásticos lineares, foi possível observar que o comportamento mecânico dos meios anisotrópicos gerais difere consideravelmente do comportamento dos meios que possuem isotropia ou até mesmo ortotropia.

Isso pode ser constatado nos primeiros exemplos do capítulo 7, observando-se as distribuições de tensões normais e tangenciais das vigas constituídas de materiais completamente anisotrópicos. Exemplos como esse, que foram estudados no desenvolvimento dos códigos, possibilitaram na análise com o MEF a constatação da necessidade de adoção de uma aproximação numérica no mínimo quadrática em deformações para se obter resultados satisfatórios em tensões no estudo de meios fortemente anisotrópicos.

Essa constatação, não mencionada na literatura consultada, é muito importante para abordagem de problemas com plasticidade, ou com outro tipo de não linearidade, onde se precisa de soluções numéricas confiáveis para tensões no sentido de se alcançar a convergência de resposta requerida. Na presente pesquisa, isso foi alcançado com a substituição, ainda no programa elástico linear, do elemento finito LST pelo elemento QST (ambos triangulares).

No exemplo 3 do capítulo 7, os bons resultados calculados e apresentados para os fatores de intensidade de tensão, sem o emprego de qualquer elemento especial para análise de trincas, evidenciam a superioridade do MEC no estudo de problemas com gradientes de tensão proeminentes, quando comparado ao MEF.

No capítulo 8, os exemplos demonstram uma outra contribuição científica importante da pesquisa que é a abordagem de problemas viscoelásticos, em meios com anisotropia geral, pelo MEC utilizando-se apenas integrais de contorno sem a utilização de aproximações de domínio (MESQUITA \& CODA, 2002). A formulação empregada, com os dois métodos, ainda possui outras inovações como a utilização de incrementos de tempo, semelhante às análises dinâmicas e a consideração diferencial direta do modelo reológico do material constituinte do problema nas equações de equilíbrio, sem o uso das funções de fluência e de relaxação. As aplicações apresentadas são relativamente simples, mas importantes para se mostrar que os programas fornecem bons resultados e possibilitam a análise do comportamento viscoelástico dos materiais 
anisotrópicos. Salienta-se que essa é uma área (teórico - experimental) ainda aberta à pesquisa.

As análises viscoplásticas presentes no capítulo 9 evidenciam a contribuição científica principal, em termos de originalidade, do trabalho. No primeiro exemplo, soluções para uma chapa quase isotrópica são apresentadas, as quais, estão coincidentes com as obtidas por MESQUITA (2002), aferindo-se assim, os programas desenvolvidos. Destaca-se nessas aplicações a utilização do critério de plastificação de TSAI \& WU (1971), para o qual, foi desenvolvido de maneira original um algoritmo de retorno, contendo as hipóteses de lei de fluxo plástico não-associativa e encruamento isotrópico, determinando-se uma equação que permite o cálculo de uma expressão fechada para o multiplicador plástico sem a utilização de processos iterativos na análise.

Na abordagem pelo MEC, outro aspecto inovador da presente pesquisa foi a solução dada para a determinação do termo livre para a análise de plasticidade. Como comentado no capítulo 5, e detalhado no Anexo 2, para a solução fundamental de CRUSE \& SWEDLOW (1971) esse termo não é conhecido analiticamente, limitando-se assim, as aplicações em plasticidade com essa solução. No trabalho, essa dificuldade foi sanada ao se calcular esse termo numericamente, estratégia essa que já consiste em contribuição ao MEC.

É importante comentar que um tempo considerável foi aplicado na tentativa de se determinar analiticamente esse termo que, na verdade, para a solução fundamental em questão, consiste de uma matriz 3 x 3 conforme pode ser observado no Anexo 2. No entanto, mesmo com a utilização de softwares de manipulação simbólica, isso não foi possível, fato esse que acabou sendo responsável pelo surgimento da estratégia de cálculo numérico.

Os dois últimos exemplos analisados no capítulo 9 são também relativamente simples, mas, pode-se afirmar que são únicos sobre o assunto. As respostas obtidas com os dois programas, em termos de tensões e de deslocamentos, são coincidentes e demonstram a eficiência dos códigos desenvolvidos. É importante observar, nos gráficos de tensões, que as tensões viscosas e elastoplásticas são complementares no sistema, conforme se observa no modelo reológico viscoplástico apresentado no capítulo 4. Deve-se novamente comentar que o modelo implementado não considera o comportamento instantâneo de deformação, mas foi perfeitamente adequado para os propósitos iniciais da pesquisa. 
Sobre a modelagem de meios reforçados com fibras com o MEF, apresentada no capítulo 10, que permite a consideração de distribuição aleatória de fibras no domínio do problema analisado, sem qualquer reordenação de malha e nem aumento dos graus de liberdade da análise, também pelo conhecimento do autor e de seu orientador, é importante dizer que é uma abordagem inédita. Como exposto, os programa comerciais mais usados na área, como ANSYS e ABAQUS, apesar de considerarem o reforço com fibras, não utilizam as mesmas hipóteses aqui empregadas.

Os resultados das aplicações feitas, especialmente os exemplos 2 e 3, mostram uma boa concordância com as soluções experimentais usadas para comparação demostrando-se, que o programa pode ser aplicado em muitos problemas práticos de Engenharia de Estruturas e ser usado em laboratórios para comparação com resultados experimentais oriundos de ensaios em materiais fibrosos oferecendo subsídios para futuras pesquisas.

Evidentemente, algumas diferenças estão presentes nos resultados apresentados, mas são perfeitamente explicáveis pelo fato dos resultados experimentais estarem cercados de fatores que nem sempre são equacionáveis. Salienta-se ainda que a formulação implementada até o presente momento não considera o deslizamento das armaduras longitudinais e nem das fibras e, portanto, uma rigidez maior das soluções numéricas é esperada.

\subsection{CONCLUSÃO GERAL E SUGESTÕES PARA PESQUISAS FUTURAS}

Analisando-se todas as aplicações apresentadas, pode-se chegar à principal conclusão do trabalho que é a de que as formulações propostas e utilizadas conseguem descrever, de maneira muito satisfatória, os fenômenos envolvidos nos problemas tratados e que os dois códigos computacionais estão funcionando perfeitamente e apresentando bons resultados, permitindo-se assim, com segurança, que possam ser utilizados por outros engenheiros na análise de problemas planos com viscoplasticidade em meios anisotrópicos, bem como, servirem de códigos iniciais de pesquisas futuras.

Desta maneira, em vista de tudo que se foi exposto neste capítulo, pode-se então concluir o trabalho apresentando algumas sugestões para trabalhos futuros. 
A presente pesquisa certamente servirá como referência para o desenvolvimento de estudos voltados à análise numérica, 2D ou 3D, de meios anisotrópicos. Um primeiro assunto que se pode sugerir é a abordagem dinâmica dos problemas aqui tratados. Para tanto, acredita-se que com simples modificações nas formulações descritas nos capítulos 4 e 5, acrescentando-se termos inerciais, pode-se chegar nas expressões algébricas necessárias.

Para o MEC, pode-se também explorar melhor a solução fundamental de CRUSE \& SWEDLOW (1971) procurando desenvolver as expressões analíticas necessárias para as integrações fundamentais à montagem das matrizes $H$ e $G$, bem como, na determinação do termo livre da plasticidade.

Para o MEF, na modelagem das fibras, pode-se trabalhar ainda no sentido de se considerar outras hipóteses na formulação como, por exemplo, deslizamento das barras. Para problemas tridimensionais, como placas e cascas, utilizando-se outros elementos finitos, as mesmas considerações sobre o posicionamento das fibras, sem aumento dos graus de liberdade, podem ser feitas. 


\section{REFERÊNCIAS BIBLIOGRÁFICAS}

ALBUQUERQUE, E.L. Análise de Problemas dinâmicos em Materiais Anisotrópicos usando o Método dos Elementos de Contorno. 2001. 120f. Tese (doutorado em engenharia mecânica) - Faculdade de Engenharia Mecânica, Universidade Estadual de Campinas, Campinas.

ALBUQUERQUE, E. L., SOLLERO, P., ALIABADI, M. H. The boundary element method applied to time dependent problems in anisotropic materials. International Journal of Solids and Structures, 2002, vol. 39, pg.1405-1422.

AL. DABBAGH, A., GOODMAN, J. R., BODIG, J. Finite element method for wood mechanics. Journal of the Structural Division, 1972, vol. 98, n.ST3, pg.569-586.

AL-ORAIMI S. K., SEIBI A. C. Mechanical characterisation and impact behaviour of concrete reinforced with natural fibres. Composite Structures, vol. 32, n. 1-4, 1995, p 165-171.

ALIABADI, M.H.; HALL, W.S.; PHEMISTER, T.G. Taylor expansions for singular kernels in the boundary element method. Int. J. Num. Meth. Eng., 1985, v. 21, p. 2221-2236.

ALIABADI, M. H.; ROOKE, D. P., Numerical Fracture Mechanics. The Netherlands, 1992, Kluwer Academic Publishers.

ASSAN, A.E. Método dos elementos finitos: primeiros passos. $1^{\text {a }}$ ed. Campinas, SP: Editora da Unicamp, 1999. 298 p.

AZIS, M. I., CLEMENTS, D. L. A boundary element method for anisotropic inhomogeneous elasticity. International Journal of Solids and Structures, 2001, vol. 38, pg.5747-5763. 
BARNETT, D. M., LOTHE, J., Synthesis of the sextic and the integral formalism for dislocations, Green’s functions and surface waves in anisotropic elastic solids. Physics Norv. 1973, vol.7, pg.13-19.

BENJUMEA, R., SIKARSKIE, D. L. On solution of plane, orthotropic elasticity problems by an integral method. Journal of Applied Mechanics, 1972, vol. 94, pg.801-808.

BENTUR, A.; MINDESS, S. Fibre reinforced cementitious composites. United Kingdom. Barking, Elsevier. 1990.

BORST R. de, FEENSTRA P. H. Studies in anisotropic plasticity with reference to the Hill criterion. International Journal for Numerical Methods in Engineering, 1990, vol.29, pg.315 - 336 .

BREBBIA, C.A., DOMINGUEZ, J. Boundary elements: an introductory course. 1.ed. McGraw-Hill Book Company, Great Britain (1989).

BREBBIA, C.A., TELLES, J. C. F., WROBEL L. C. Boundary elements techniques - Theory and applications in engineering. (1984), 464p.

BRÜNIG, M. Nonlinear analysis and elastic-plastic behavior of anisotropic structures. Finite Elements in Analysis and Design, 1995, vol. 20, pg.155-177.

BUI, H.D. Some remarks about the formulation three-dimensional thermoelastoplastic problems by integral equations, Int. J. Solids Structures, 1978, v.14, p.935-939.

CALLISTER Jr., W. D. Materials science and engineering. New York, John Wiley \& Sons, 1985.

CHEN, W.F., SALEEB. A. Constitutive Equations for Engineering Materials. New York, John Wiley and sons, 1982. v.1: Elasticity and Modeling 396p.

CHIU, Y. T., WU K. C. Analysis for elastic strips under concentrated loads. Journal of Applied Mechanics, 1998, vol. 65, pg.627-634. 
CHUEIRI, L.H.M. (1994). Formulação do método dos elementos de contorno para análise elastoplástica de placas. São Carlos. Tese (Doutorado) - Escola de Engenharia de São Carlos, Universidade de São Paulo.

CISILINO, A.P. Boundary element formulations for 3D analysis of fatigue crack growth in linear and non-linear fracture mechanics. UK, 1997, 234p. Thesis (Doctor of Philosophy) - Wessex Institute of Technology, University of Wales.

CLOUSTON P. L., LAM F. Computational modeling of strand-based wood composites. Journal of Engineering Mechanics, 2001, vol.127, n.8, pg.844 - 851.

CODA, H. B. Análise da vibração livre de meios elásticos bidimensionais pelo método dos elementos de contorno. São Carlos, 1990, 200p. Dissertação (Mestrado) - Escola de Engenharia de São Carlos, Universidade de São Paulo.

CODA, H. B. , VENTURINI W. S. On the coupling of 3D bem and fem frame model applied to elastodynamic analysis. International Journal of Solids and Structures, vol.36, n.31-32, November 1999, p.4789-4804.

CODA, H.B. Contribuição à análise dinâmica transiente de meios contínuos pelo Método dos Elementos de Contorno. São Carlos, 2000, 381p. Tese (Livredocência) - Escola de Engenharia de São Carlos, Universidade de São Paulo.

CODA, H.B. Dynamic and static non-linear analysis of reinforced media: a BEM/FEM coupling approach. Computers \& Structures, vol. 79, n. 31, December 2001, p.2751-2765.

COURAGE, W. M. G., SCHREURS P. J. G. Effective Material parameters for composites with randomly oriented short fibers. Computers \& Structures, 1992, vol. 44, n.6, pg.1179-1185.

CRUSE, T. A., RIZZO F. J. A direct formulation and numerical solution of the general transient elastodynamic problem - I. Journal Mathematics Analysis Applied, 1968, vol.22, pg.244-259.

CRUSE, T. A. Recent advances in boundary element analysis methods. Computer Methods in Applied Mechanics and Engineering, 1987, vol.62, pg.227-244. 
CRUSE, T. A., SWEDLOW, J. L. Interactive program for analysis and design problems in advanced composites. Technical Report, Carnegie-Mellon University, Report AFLM-TR-71-268, 1971.

DAVIS B., GRAMANN P., RIOS A. Effect of Fiber Orientation Anisotropies on the Structural Performance of Molded FRP Composite Parts. The Madison Group: Polymer Processing Research Corporation, 2002.

DEB, A., BANERJEE P. K. BEM for general anisotropic 2D elasticity using particular integrals. Communications in Applied Numerical Methods, 1990, vol.6, pg.1721-1738.

DEB, A., HENRY D. P., WILSON R. B. Alternate BEM formulations for 2D and 3D anisotropic thermo-elasticity. International Journal of Solids and Structures, 1991, vol.27, pg.111-119.

DEB, A. Boundary element analysis of anisotropic bodies under thermo-mechanical body force loadings. Computers \& Structures, 1996, vol.58, n.4, pg.715-726.

DING, H., LIANG, J. The fundamental solutions for transversely isotropic piezoelectricity and boundary element method. Computers \& Structures, 1999, vol.71, pg.447-455.

ESHELBY, J. D., READ, W. T., SHOCKLEY, W. Anisotropic elasticity with applications to dislocation theory. Acta Metallica, 1953, vol.1, pg.251-259.

FEENSTRA, P.H.; BORST, R. de. A composite plasticity model for concrete. Int. J. Solids Structures, 1996, v.33, pp. 707-703.

FERREIRA, A.J.M., MARQUES, A.T., SÁ C., BARBOSA J.A.T. Validação Experimental de modelos de cálculo de estruturas sandwich. Revista Mecânica Experimental - APAET. Assoc. Portuguesa de Análise Experimental de Tensões, 1999, n.4, pp. 101-109. 
FIGUEIRAS, J.A. Ultimate load analysis of anisotropic and reinforced concrete plates and shells. Swansea, 1983, Thesis (Ph. D) - Department of Civil Engineering, University College of Swansea.

FIGUEIREDO, A.D. Concreto com fibras de aço. Apostila publicada pela Escola Politécnica da Universidade de São Paulo. São Paulo, 2000.

FREDHOLM, I. Sur une classe d'equations fonctionelles. Acta Math, 1903, vol.27, pp. 365-390.

FUDOLI, C.A. Formulação do método dos elementos de contorno e plasticidade com gradiente. São Carlos, 1999, Tese (Doutorado) - Escola de Engenharia de São Carlos, Universidade de São Paulo.

FURLAN Jr, S. Vigas de concreto com taxas reduzidas de armadura de cisalhamento: influência do emprego de fibras curtas e de protensão. São Carlos, 1999, 360 p, Tese (Doutorado) - Escola de Engenharia de São Carlos, Universidade de São Paulo.

FUSCO, P.B. Resistência dos Materiais Anisotrópicos. I Encontro Regional em Madeiras e em Estruturas de Madeira. p.159-188. São Paulo. nov.1993.

GIAVOTTO, V., BORRI, M., MANTEGAZZA, P., GHIRINGHELLI, G., CARMASCHI, V., MAFFIOLI, G. C., MUSSI, F. Anisotropic beam theory and applications. Computers \& Structures, 1983, vol.16, n.1-4, pg.403-413.

GIBSON, R. F. Principles of composite material mechanics. McGraw-Hill Publ., New York, 1994, 425 p.

GREEN, A.E. A note on stress systems in aelotropic materials. Philosophical Magazine, 1943, vol. 34, pg.416-418.

GREEN, A.E., ZERNA, W. Theoretical Elasticity. Glasgow: Clarendon Press, 442 p. 1954.

HASHIN, Z. Plane Anisotropic Beams. Journal of Applied Mechanics, p.257-62. 1967. 
HASHIN Z., ROTEM A. A fatigue failure criterion for fiber-reinforced materials. Journal of Composite Materials, 1973, vol.7, pg.448 - 454.

HASHIN Z. Failure criteria for unidirectional fiber composites. Journal of Applied Mechanics, 1980, vol.47, pg.329 - 334.

HILL, R. A theory of the yielding and plastic flow of anisotropic metals. Proc. Roy. Soc.(London), Series A, 1948, vol.193, pg.281 - 297.

HOFFMAN, O. The brittle strength of orthotropic materials. Journal of Composite Materials, 1967, vol.1, pg.200 - 210.

HOLANDA, K. M. A. Análise dos mecanismos resistentes e das similaridades de efeitos da adição de fibras de aço na resistência e na ductilidade à punção de lajes cogumelo e ao cisalhamento de vigas de concreto. São Carlos, 2002, 302p. Tese (Doutorado) - Escola de Engenharia de São Carlos, Universidade de São Paulo.

HOLMBERG, S., PERSSON, K., PETERSSON, H. Nonlinear mechanical behavior and analysis of wood and fibre materials. Computers and Structures, 1999, vol. 72, pg.459-480.

HU H.T., LIN F. M., JAN Y. Nonlinear finite element analysis of reinforced concrete beams strengthened by fiber-reinforced plastics. Composite Structures, vol.63, n. 3-4, February-March 2004, p.271-81.

HYER, M. W. Stress Analysis of fiber-reinforced Composite Materials. McGrawHill Publ., New York, 1997, 627 p.

KHAROUF N., McCLURE G., SMITH I. Elasto-plastic modeling of wood bolted connections. Computers \& Structures, 2003, vol.81, pg.747 - 754.

KÖGL, M., GAUL, L. A boundary element method for transient piezoeletric analysis. Engineering Analysis with Boundary Elements, 2000, vol.24, pg.591-598.

KOLAKOWSKI Z. On some aspects of the modified Tsai-Wu criterion in thinwalled composite structures. Thin Walled Structures, 2003, vol.41, pg.357 - 374. 
KHUNTIA, M., STOJADINOVIC, B., GOEL, S.C. Shear Strength of normal and high-strength fiber reinforced concrete beams without stirrups. ACI Structural Journal, 2000, vol.96, n.2, pg.282-89, march-april.

KUPRADZE, V. D. Potential methods in theory of elasticity. Jerusalém, Israel Program for Scientific Translations, 1965.

LEE, K. J., MAL, A. K. A boundary element method for plane anisotropic elastic media. Journal of Applied Mechanics, 1990, vol.57, pg.600-606.

LEITÃO, V.; ALIABADI, M.H.; ROOKE, D.P. The dual boundary element formulation for elastoplastic fracture mechanics. Int. J. Num. Meth. Eng., 1995, v. 38, p. 315-333.

LEITE L.G.S., CODA H. B., VENTURINI, W.S. Two-dimensional solids reinforced by thin bars using the boundary element method. Engineering Analysis with Boundary Elements, Vol. 27, n. 3, March 2003, p.193-201

LEKHNITSKII, S.G. Theory of Elasticity of an Anisotropic Body. $1^{\mathrm{a}}$ ed. Moscou: Mir, 1963. 430p.

LEKHNITSKII, S.G., TSAI, S.W., CHERONT, T. Anisotropic Plates. $1^{\mathrm{a}}$ ed. New York: Gordon and Breach Science Publishers, 1968. 534p.

LEMAITRE, J. and CHABOCHE, J.L. Mechanics of Solids, 1990, Cambridge University Press.

LIM, D. H., OH, B. H. Experimental and theoretical investigation on the shear of steel fiber reinforced concrete beams. Engineering Structures, 2000, vol.21, pg.937-44.

LIU, Y. J. On the simple-solution method and non-singular nature of the BIE/BEM a review and some new results. Engineering Analysis with Boundary Elements, 2000, vol. 24, pg.789-795 
MACIEL, D. N. Determinação dos fatores de intensidade de tensão estáticos e dinâmicos via MEC com integração analítica em coordenadas locais. São Carlos, 2003. Dissertação (Mestrado) - Escola de Engenharia de São Carlos, Universidade de São Paulo.

MAGAGNIN FILHO, Nilson. Placas laminadas em materiais compostos de fibras longas: propriedades termoelásticas dos materiais constituintes; propriedades equivalentes das lâminas; critérios de ruptura e análise pelo método dos elementos finitos. São Carlos, 1996. Dissertação (Mestrado) - Escola de Engenharia de São Carlos, Universidade de São Paulo.

MAHAJERIN, E., SIKARSKIE, D. L. Boundary element study of a loaded hole in na orthotropic plate. Journal of Composite Materials, 1986, vol.20, pg.375-389.

MASCIA, N.T. Considerações a respeito da Anisotropia na Madeira. 1991. 293p. Tese (doutorado em engenharia civil)- EESC-USP, São Carlos.

MELESH, R. J. Structural analysis of solids. Journal of the Structural Division, v89, n. ST4, Aug. 1963.

MENDELSON, A. Boundary integral methods in elasticity and plasticity, Report No. NASA TN D-7418, 1973, NASA.

MESQUITA, A.D. Uma formulação do método dos elementos finitos aplicada à análise elastoplástica de cascas. São Carlos, 1998, 144p. Dissertação (Mestrado) Escola de Engenharia de São Carlos, Universidade de São Paulo.

MESQUITA, A. D. Novas metodologias e formulações para o tratamento de problemas inelásticos com acoplamento MEC/MEF progressivo. 2002. Tese (Doutorado) - Escola de Engenharia de São Carlos, Universidade de São Paulo, USP.

MESQUITA A. D., CODA, H. B. An alternative time integration procedure for Boltzmann viscoelasticity: A BEM approach. Computers \& Structures, 2001, vol. 79, n.16 pg.1487-1496. 
MESQUITA, A.D.; CODA, H.B. Alternative Kelvin viscoelastic procedure for finite elements. Applied Mathematical Modelling, 2002, vol.26, pp. 501-516.

MI, Y.; ALIABADI, M.H. Dual boundary element method for three dimensional fracture analysis. Engn. Analysis with Boundary Elements, 1992, v.10, p. 161-171.

MIKHLIN, S. G. Integral equations. Oxford, Pergamon Press, 1957.

MUNAIAR NETO, J. Um estudo da formulação de modelos constitutivos viscoelásticos e elasto-viscoplásticos e do emprego de algoritmos implícitos para a sua integração numérica. São Carlos, 1998, 214p. Tese (Doutorado) - Escola de Engenharia de São Carlos, Universidade de São Paulo.

MUSKHELISHVILI, N. I. Some basic problems of the mathematical theory of elasticity. Holland, Noordhoff, 1953.

NOACK ,D., ROOTH, von W. On the theory of elasticity of orthotropic material. Wood Science and Technology, v10, p.97-110, 1976.

ORTIZ, M. e POPOV, E.P. (1985). Accuracy and stability of integration algorithms for elastoplastic constitutive relations. Int. J. Numer. Meth. Engng., v. 21, pp. 1561-1576.

PAN, Y. C., CHOU, T. W. Point force solution for an infinite transversely isotropic solid. Journal of Applied Mechanics, december 1976, pg.608-612.

PAN, E., AMADEI, B. A 3-D boundary element formulation of anisotropic elasticity with gravity. Applied Mathematics Modelling, 1996, vol. 20, pg.114-120.

PAN, E., YUAN, F. G. Three-dimensional Green's functions in anisotropic bimaterials. International Journal of Solids and Structures, 2000, vol. 37, pg.53295351.

PAN, E., YANG, B., CAI, G., YUAN, F. G. Stress analysis around holes in composite laminates using boundary element method. Engineering Analysis with Boundary Elements, 2001, vol. 25, pg.31-40. 
PADHI, G. S., SHENOI, R. A., MOY, S. S. J., HAWKINS, G. L. A quadratic boundary element implementation in orthotropic elasticity using the real variable approach. Communications in Numerical Methods in Engineering, 2000, vol. 16, pg.257-266.

PARÍS, F.; CAÑAS, J. Boundary Element Method fundamentals and applications. Oxford, 1997, Oxford University Press.

PEREZ, M. M., WROBEL, L. C. An integral-equation formulation for anisotropic elastostatics. Journal of Applied Mechanics, 1996, vol. 63, pg.891-902.

POPESCU, B., HODGES, D.H. On asymptotically correct Timoshenko-like anisotropic beam theory. International Journal of Solids and Structures, 2000, vol.37, pg.535-558.

PORTELA , A.; ALIABADI, M. H.; ROOKE, D. P. Dual boundary element method: Efficient implementation for cracked problems. International Journal Numerical Methods Engineering, 1992, v.33, p.1269-1287.

QIN, Z., LIBRESCU, L. On a shear-deformable theory of anisotropic thin-walled beams: further contribution and validations. Composites Structures, 2002, vol.56, pg.345-358.

RAJU, I. S., SISTIA, R., KRISHNAMURTHY, T. An efficient boundary element method for computing accurate stress in two-dimensional anisotropic problems. Computers \& Structures, 1996, vol.59, n.3, pg.453-462.

RAVEENDRANATH, P., SINGH, G., PRADHAN, B. Application of coupled polynomial displacement fields to laminated beam elements. Computers \& Structures, 2000, vol.78, pg.661-670.

RICCARDELLA, P.C. A implementation of the boundary integral technique for planar problems in elasticity and elastoplasticity, SM-73-10, Dept. Mech. Engng., Carnegie Mellon University, 1973, Pittsburg.

RIZZO, F. J. An integral equation approach to boundary value problems of classical elastostatics. Quarterly Journal of Applied Mathematics, 1967, vol.25, pg.83-95. 
RIZZO, F. J., SHIPPY, D. J. A method for stress determination in plane anisotropic elastic bodies. Journal of Composite Materials, 1970, vol.4, pg. 36.61

SALES, M. A., GRAY, L. J. Evaluation of the anisotropic Green's function and its derivatives. Computers \& Structures, 1998, vol.69, pg.247-254.

SCHCLAR, N.A. Anisotropic Analysis Using Boundary Elements. Topics in Engineering. Computational Mechanics Publications, 1994, 142p.

SCHCLAR, N. A., PARTRIDGE, P. W. 3D anisotropic elasticity with BEM using isotropic fundamental solution. Engineering Analysis with Boundary Elements, 1993, vol. 11, pg.137-144.

SHIH C. F., LEE D. Further developments in anisotropic plasticity. Journal of Engineering Materials and Technology, 1978, vol.100, pg.294 - 302.

SILVERMAN, I. K. Orthotropic beams under polynomial loads. ASCE, Journal of the Engineering Mechanics Division, V.90, EM5, p. 293-319, oct. 1964.

SNYDER, M. D., CRUSE, T. A. Boundary-integral equation analysis of cracked anisotropic plates. International Journal of Fracture, 1975, vol. 11, n.2, pg.315328.

SOLLERO, P., ALIABADI, M. H. Fracture mechanics analysis of anisotropic plates by the boundary element method. International Journal of Fracture, 1993, vol. 64, pg.269-284.

STROH, A. N. Dislocations and cracks in anisotropic elasticity. Philosophical Magazine, 1958, vol.3, pg.625-646.

STROH, A. N. Steady state problems in anisotropic elasticity. Journal Mathematics Physics (Cambridge, MA), 1962, vol.41, pg.77-103.

SUN X., LIU Y., CEN Z. The formulations of boundary element method on plane plastic problems for orthotropic materials. In: Proceedings of the Third International Conference on BeTeQ (Boundary Element Techniques), Sept. 10-12, 2002, Beijing, China 
SYNGE, J. L. The Hypercircle in Mathematical Physics. Cambridge University Press, Cambridge 1957, pg.411-413.

TAN, C. L., GAO, Y. L. Boundary integral equation fracture mechanics analysis of plane orthotropic bodies. International Journal of Fracture, 1992, vol.53, pg.343365.

TAKEYA, T., $2^{\circ}$ Relatório de Iniciação Científica II, FAPESP (1972).

TELLES, J.C.F.; BREBBIA, C.A. (1982). Elastic/viscoplastic problems using boundary elements. Int. J. Mech. Sci., v.4, n.10, p.605-618.

TELLES, J.C.F.; CARRER, J.A.M. Static and transient dynamic nonlinear stress analysis by the boundary element method with implicit techniques. Engineering Analysis with Boundary Elements, 1994, v.14, n.1, p.65-74.

TING, T.C.T. Anisotropic Elasticity. Oxford University Press, 1996.

TING, T. C. T. Recent developments in anisotropic elasticity. International Journal of Solids and Structures, 2000, vol.37, pg.401-409.

TITA, V. Análise dinâmica teórica e experimental de vigas fabricadas a partir de materiais compósitos poliméricos reforçados. São Carlos, 1999, 125p. Dissertação (Mestrado) - Escola de Engenharia de São Carlos, Universidade de São Paulo.

TONON, F., PAN, E., AMADEI, B. Green's functions and boundary method formulation for 3D anisotropic media. Computers \& Structures, 2001, vol.79, pg.469-482.

TSAI S. W. Strength theories of filamentary structures. In: R. T. Schwartz and H. S. Schwartz (eds.), Fundamental Aspects of Fiber Reinforced Plastic Composites, 311, Chap 1, Wiley Interscience, New York, 1968.

TSAI S. W., WU E. M. A general theory of strength for anisotropic materials. Journal of Composite Materials, 1971, vol.5, pg.58 - 80. 
TURNER, M. J., CLOUGH, R. W., MARTIN, H. C., TOPP, J. L. Stiffness and deflection analysis of complex structures. Journal Aeronautic Science, 1956, vol.23, n.9, pg. 805-823.

VABLE, M., SIKARSKIE, D. L. Stress analysis in plane orthotropic material by the boundary element method. International Journal Solids Structures, 1988, vol. 24, n.1, pg.1-11.

VANALLI, L. Análise das Distribuições de Tensões e de Deformações em Vigas Anisotrópicas. 2001. 120f. Dissertação (mestrado em engenharia civil) Faculdade de Engenharia Civil, Universidade Estadual de Campinas, Campinas.

VANALLI, L., PACCOLA, R. R., CODA, H. B. Analysis of plane anisotropic structures by FEM and BEM. In: 24nd. Iberian Latin-American Congress on Computational Methods in Engineering - XXIV CILAMCE, Tipo de Mídia: CDROOM, Ouro Preto - MG, 2003.

VANALLI, L., MACIEL, D. N., PACCOLA, R. R., CODA, H. B. Alternative BEM stress intensity factor analysis of anisotropic and isotropic media. In: 24nd. Iberian Latin-American Congress on Computational Methods in Engineering - XXIV CILAMCE, Tipo de Mídia: CD-ROOM, Ouro Preto - MG, 2003.

VENTURINI, W.S. Application of the boundary element method to solve geomechanical problems. 1982, Phd Thesis, WIT, UK.

VENTURINI, W. S. Um estudo sobre o método dos elementos de contorno e suas aplicações em problemas de engenharia. 1988. Tese (Livre-Docência) - Escola de Engenharia de São Carlos, Universidade de São Paulo, USP.

VOGEL, S. M., RIZZO, F. J. An integral equation formulation of three dimensional anisotropic elastostatic boundary value problems. Jounal of Elasticity, 1973, vol.3, pg.203-216.

WANG, C. Y. Two-dimensional elastostatic Green's functions for general anisotropic solids and generalization of Stroh's formalism. International Journal of Solids and Structures, 1994, vol.31, n.19, pg.2591-2597. 
WILSON, E. L. Structural analysis of axisymmetric solids. AIAA Journal, 1965, vol. 3, pg.2269-2274.

WILSON, R. B, CRUSE, T. A. Efficient implementation of anisotropic three dimensional boundary integral equation stress analysis. International Journal for Numerical Methods in Engineering, 1978, vol.12, pg.1383 - 1397.

WU, K. C. The elastic fields of line force or dislocations in an anisotropic wedge. International Journal of Solids and Structures, 1998, vol.35, n.26-27, pg.34833495.

WYLIE C. R., BARRETT L. C. Advanced engineering mathematics - sixth edition. McGraw-Hill, 1995, 1362p.

YILDIZ, H., SARIKANAT, M. Finite-element analysis of thick composite beams and plates. Composites Science and Technology, 2000, vol. 61, pg.1723-1727. 
ANEXOS 


\section{ANEXO 1 - DETALHAMENTo da SOLUÇÃO FUNDAMENTAL DE CRUSE \& SWEDLOW (1971)}

Neste Anexo, a solução fundamental anisotrópica, desenvolvida por CRUSE \& SWEDLOW (1971), é deduzida de maneira detalhada fazendo-se uso do formalismo de LEKHNITSKII (1963) e da teoria das funções complexas. Para tanto, considera-se primeiramente o tensor das tensões escrito em termos das funções de tensão de Airy $\left(F\left(x_{1}, x_{2}\right)\right)$ dadas por:

$$
\begin{aligned}
& \sigma_{11}=F,_{22}+v \\
& \sigma_{22}=F,_{11}+v \\
& \sigma_{12}=-F_{,_{12}}
\end{aligned}
$$

onde $v$ é uma função potencial que, considerando o problema estático, tem valor nulo.

Essas funções satisfazem as equações de equilíbrio:

$$
\sigma_{i j},_{j}=0
$$

Expressando-se as deformações em termos das funções de Airy:

$$
\left\{\begin{array}{l}
\varepsilon_{1} \\
\varepsilon_{2} \\
\varepsilon_{6}
\end{array}\right\}=\left[\begin{array}{lll}
S_{11} & S_{12} & S_{16} \\
S_{12} & S_{22} & S_{26} \\
S_{16} & S_{26} & S_{66}
\end{array}\right] \cdot\left\{\begin{array}{c}
F_{, 22} \\
F_{, 11} \\
-F_{,_{12}}
\end{array}\right\}
$$


pode-se diferencia-las como segue:

$$
\begin{aligned}
& \varepsilon_{1,22}=S_{11} \cdot F,_{2222}+S_{12} \cdot F_{,_{1122}}-S_{16} \cdot F_{{ }_{1222}} \\
& \varepsilon_{2,11}=S_{12} \cdot F,_{2211}+S_{22} \cdot F,_{1111}-S_{26} \cdot F,_{1211} \\
& \varepsilon_{6,12}=S_{16} \cdot F,_{2212}+S_{26} \cdot F_{{ }_{1112}}-S_{66} \cdot F_{r_{1212}}
\end{aligned}
$$

Substituindo-se as expressões (A1.6 - A1.8) nas equações de compatibilidade:

$$
\varepsilon_{1,22}+\varepsilon_{2,11}=\varepsilon_{6,12}
$$

obtém-se:

$$
S_{11} F_{, 2222}-2 S_{16} F_{, 1222}+\left(2 S_{12}+S_{66}\right) F_{,_{1122}}-2 S_{26} F_{,_{1112}}+S_{22} F_{,_{1111}}=0
$$

que é a equação diferencial parcial de quarta ordem que modela um problema elástico anisotrópico nos moldes do formalismo de LEKHNITSKII (1963) e que tem como solução a função de tensão de Airy $\left(F\left(x_{1}, x_{2}\right)\right)$. Esta equação pode ser integrada na sua forma geral escrevendo-a simbolicamente com o uso de quatro operadores diferenciais lineares:

$$
\Delta_{1} \Delta_{2} \Delta_{3} \Delta_{4} F=0
$$

onde $\Delta$ designa a operação:

$$
\Delta_{k}=\frac{\partial}{\partial x_{2}}-\mu_{k} \frac{\partial}{\partial x_{1}} \quad(k=1,2,3,4)
$$

e $\mu_{k}$ são as raízes da equação característica (LEKHNITSKII et al., 1968):

$$
S_{11} \mu^{4}-2 S_{16} \mu^{3}+\left(2 S_{12}+S_{66}\right) \mu^{2}-2 S_{26} \mu+S_{22}=0
$$

Segundo LEKHNITSKII et al. (1968), as raízes da Equação (A1.13) são sempre complexas ou imaginárias puras, não ocorrendo raízes reais para o caso de qualquer corpo elástico real com constantes $S_{11}, 2 S_{12}+S_{66}$ e $S_{22}$ finitas e diferentes de zero. Essas raízes complexas recebem a designação $\mu_{1}, \mu_{2}, \bar{\mu}_{1}$ e $\bar{\mu}_{2}$. Dois casos de combinações das raízes são possíveis, dependendo-se das relações entre as constantes elásticas:

$\left.1^{0}\right)$ As raízes são todas diferentes:

$$
\mu_{1}=\alpha+\beta \cdot i, \mu_{2}=\gamma+\delta \cdot i, \bar{\mu}_{1}=\alpha-\beta \cdot i, \bar{\mu}_{2}=\gamma-\delta \cdot i
$$


onde $\alpha, \beta, \gamma, \delta \in \mathrm{R}$ e $\beta>0$ e $\delta>0$.

$2^{\circ}$ ) As raízes são pares conjugados:

$$
\mu_{1}=\mu_{2}=\alpha+\beta \cdot i \text { e } \bar{\mu}_{1}=\bar{\mu}_{2}=\alpha-\beta \cdot i
$$

As raízes $\mu_{1}$ e $\mu_{2}$ são parâmetros complexos que podem ser considerados como números que caracterizam o grau de anisotropia em problemas planos. De acordo com seus valores, pode-se avaliar o quanto um dado corpo anisotrópico difere de um isotrópico, para o qual, $\mu_{1}=\mu_{2}=i$ e $\left|\mu_{1}\right|=\left|\mu_{2}\right|=1$.

A partir da Equação (A1.13) pode-se resolver o problema elástico evitando-se, dessa forma, de se resolver diretamente a equação diferencial (A1.10). Para tanto é preciso proceder a seguinte mudança de variável onde o domínio $\Omega$, do problema em questão, é mapeado num plano complexo:

$$
z_{k}=x_{1}+\mu_{k} x_{2} \quad(k=1,2)
$$

Assim, procurando-se obter as componentes de tensão em função das novas variáveis complexas, é preciso lembrar que a função de tensão é real e pode ser escrita como uma combinação linear de funções de tensão complexas:

$$
F\left(x_{1}, x_{2}\right)=2 \operatorname{Re}\left[F_{1}\left(z_{1}\right)+F_{2}\left(z_{2}\right)\right]
$$

onde Re é a designação para a parte real de qualquer expressão complexa. Lembrandose ainda que as componentes de tensão são dadas de acordo com a função de Airy:

$$
\sigma_{11}=\frac{\partial^{2} F}{\partial x_{2}^{2}} ; \quad \sigma_{22}=\frac{\partial^{2} F}{\partial x_{1}^{2}} ; \quad \sigma_{12}=-\frac{\partial^{2} F}{\partial x_{1} \partial x_{2}}
$$

é possível obtê-las, considerando a Equação (A1.17), por meio da regra da cadeia:

$$
\begin{aligned}
& \frac{\partial F_{1}}{\partial x_{1}}=\frac{d F_{1}}{d z_{1}} \cdot \frac{d z_{1}}{d x_{1}}+\frac{d F_{1}}{d z_{2}} \cdot \frac{d z_{2}}{d x_{1}}=\frac{d F_{1}}{d z_{1}} \\
& \frac{\partial F_{2}}{\partial x_{1}}=\frac{d F_{2}}{d z_{1}} \cdot \frac{d z_{1}}{d x_{1}}+\frac{d F_{2}}{d z_{2}} \cdot \frac{d z_{2}}{d x_{1}}=\frac{d F_{2}}{d z_{2}} \\
& \frac{\partial F_{1}}{\partial x_{2}}=\frac{d F_{1}}{d z_{1}} \cdot \frac{d z_{1}}{d x_{2}}+\frac{d F_{1}}{d z_{2}} \cdot \frac{d z_{2}}{d x_{2}}=\mu_{1} \frac{d F_{1}}{d z_{1}}
\end{aligned}
$$




$$
\frac{\partial F_{2}}{\partial x_{2}}=\frac{d F_{2}}{d z_{1}} \cdot \frac{d z_{1}}{d x_{2}}+\frac{d F_{2}}{d z_{2}} \cdot \frac{d z_{2}}{d x_{2}}=\mu_{2} \frac{d F_{2}}{d z_{2}}
$$

Assim, depois de se efetuar também a segunda derivada tem-se:

$$
\begin{aligned}
& \frac{\partial^{2} F}{\partial x_{1}^{2}}=2 \operatorname{Re}\left[\frac{d^{2} F_{1}}{d z_{1}^{2}}+\frac{d^{2} F_{2}}{d z_{2}^{2}}\right]=\sigma_{22} \\
& \frac{\partial^{2} F}{\partial x_{2}^{2}}=2 \operatorname{Re}\left[\mu_{1}^{2} \frac{d^{2} F_{1}}{d z_{1}^{2}}+\mu_{2}^{2} \frac{d^{2} F_{2}}{d z_{2}^{2}}\right]=\sigma_{11} \\
& \frac{\partial^{2} F}{\partial x_{1} \partial x_{2}}=2 \operatorname{Re}\left[\mu_{1} \frac{d^{2} F_{1}}{d z_{1}^{2}}+\mu_{2} \frac{d^{2} F_{2}}{d z_{2}^{2}}\right]=-\sigma_{12}
\end{aligned}
$$

Analisando-se agora as componentes de deslocamento $u_{1}$ e $u_{2}$, é preciso substituir as Equações (A1.23- A1.25) nas equações constitutivas (A1.5) e assim:

$$
\begin{aligned}
& \varepsilon_{11}=S_{11} \sigma_{11}+S_{12} \sigma_{22}+S_{16} \sigma_{12} \\
& \varepsilon_{11}=\frac{d u_{1}}{d x_{1}}=2 \operatorname{Re}\left[\begin{array}{c}
\left(S_{11} \mu_{1}^{2}+S_{12}-S_{16} \mu_{1}\right) \frac{d^{2} F_{1}}{d z_{1}^{2}}+ \\
\left.\left(S_{11} \mu_{2}^{2}+S_{12}-S_{16} \mu_{2}\right) \frac{d^{2} F_{2}}{d z_{2}^{2}}\right]
\end{array}\right.
\end{aligned}
$$

Para $\varepsilon_{22}$ :

$$
\varepsilon_{22}=\frac{d u_{2}}{d x_{2}}=2 \operatorname{Re}\left[\begin{array}{l}
\left(S_{12} \mu_{1}^{2}+S_{22}-S_{26} \mu_{1}\right) \frac{d^{2} F_{1}}{d z_{1}^{2}}+ \\
\left(S_{12} \mu_{2}^{2}+S_{22}-S_{26} \mu_{2}\right) \frac{d^{2} F_{2}}{d z_{2}^{2}}
\end{array}\right]
$$

Organizando-se as expressões anteriores:

$$
\begin{aligned}
& \frac{d u_{1}}{d x_{1}}=2 \operatorname{Re}\left[q_{11} \frac{d^{2} F_{1}}{d z_{1}^{2}}+q_{12} \frac{d^{2} F_{2}}{d z_{2}^{2}}\right] \\
& \frac{d u_{2}}{d x_{2}}=2 \operatorname{Re}\left[q_{21} \frac{d^{2} F_{1}}{d z_{1}^{2}}+q_{22} \frac{d^{2} F_{2}}{d z_{2}^{2}}\right]
\end{aligned}
$$

onde: 


$$
q_{i k}=\left[\begin{array}{l}
S_{11} \mu_{k}^{2}+S_{12}-S_{16} \mu_{k} \\
S_{12} \mu_{k}+S_{22} / \mu_{k}-S_{26}
\end{array}\right]
$$

é a matriz dos parâmetros complexos (CRUSE \& SWEDLOW, 1971).

Integrando-se as Equações (A1.29) e (A1.30) e desprezando-se as parcelas referentes ao movimento de corpo-rígido, tem-se então as componentes de deslocamento:

$$
\begin{aligned}
& u_{1}=2 \operatorname{Re}\left[q_{11} \frac{d F_{1}}{d z_{1}}+q_{12} \frac{d F_{2}}{d z_{2}}\right] \\
& u_{2}=2 \operatorname{Re}\left[q_{21} \frac{d F_{1}}{d z_{1}}+q_{22} \frac{d F_{2}}{d z_{2}}\right]
\end{aligned}
$$

De posse das componentes de tensão e de deslocamentos, escreve-se os dois sistemas de equações de equilíbrio para o problema bidimensional.

$$
\begin{gathered}
\sigma_{k 11,1}+\sigma_{k 12,2}=-\delta_{k 1} \cdot \delta(s, q) \\
\sigma_{k 21,1}+\sigma_{k 22,2}=-\delta_{k 2} \cdot \delta(s, q)
\end{gathered}
$$

Com a propriedade da distribuição Delta de Dirac, integram-se as Equações (A1.34) e (A1.35) sobre um domínio real $\Omega$ que contém o ponto fonte "s”, resultando:

$$
\begin{aligned}
& \int_{\Omega}\left(\sigma_{k 11,1}+\sigma_{k 12,2}\right) d \Omega=-\delta_{k 1}=\int_{\Gamma}\left(\sigma_{k 11} \eta_{1}+\sigma_{k 12} \eta_{2}\right) d \Gamma=-\delta_{k 1} \\
& \int_{\Omega}\left(\sigma_{k 21,1}+\sigma_{k 22,2}\right) d \Omega=-\delta_{k 1}=\int_{\Gamma}\left(\sigma_{k 21} \eta_{1}+\sigma_{k 22} \eta_{2}\right) d \Gamma=-\delta_{k 2}
\end{aligned}
$$

As Equações (A1.36) e (A1.37) podem ser reescritas em uma notação incompleta no intuito de se descobrir suas primitivas (WYLIE \& BARRETT, 1995):

$$
\begin{aligned}
& \int_{L} \sigma_{k 11} d x_{2}-\int_{L} \sigma_{k 12} d x_{1}=-\delta_{k 1} \\
& \int_{L} \sigma_{k 21} d x_{2}-\int_{L} \sigma_{k 22} d x_{1}=-\delta_{k 2}
\end{aligned}
$$

onde " $L$ ” representa limites hipotéticos.

Escrevendo-se as componentes de tensão segundo as relações (A1.23- A1.25) tem-se: 


$$
\begin{array}{r}
\int_{L} 2 \operatorname{Re}\left[\mu_{1}^{2} \frac{d^{2} F_{k 1}}{d z_{1}^{2}}+\mu_{2}^{2} \frac{d^{2} F_{k 2}}{d z_{2}^{2}}\right] d x_{2}+ \\
\int_{L} 2 \operatorname{Re}\left[\mu_{1} \frac{d^{2} F_{k 1}}{d z_{1}^{2}}+\mu_{2} \frac{d^{2} F_{k 2}}{d z_{2}^{2}}\right] d x_{1}=-\delta_{k 1} \\
-\int_{L} 2 \operatorname{Re}\left[\mu_{1} \frac{d^{2} F_{k 1}}{d z_{1}^{2}}+\mu_{2} \frac{d^{2} F_{k 2}}{d z_{2}^{2}}\right] d x_{2}- \\
\int_{L} 2 \operatorname{Re}\left[\frac{d^{2} F_{k 1}}{d z_{1}^{2}}+\frac{d^{2} F_{k 2}}{d z_{2}^{2}}\right] d x_{1}=-\delta_{k 2}
\end{array}
$$

Seguindo as relações diferenciais (A1.19 - A1.22) pode-se se escrever as primitivas:

$$
\begin{aligned}
& 2 \operatorname{Re}\left[\mu_{1} \frac{d F_{k 1}}{d z_{1}}+\mu_{2} \frac{d F_{k 2}}{d z_{2}}\right]_{L}+2 \operatorname{Re}\left[\mu_{1} \frac{d F_{k 1}}{d z_{1}}+\mu_{2} \frac{d F_{k 2}}{d z_{2}}\right]_{L}=-\delta_{k 1} \\
& 2 \operatorname{Re}\left[\frac{d F_{k 1}}{d z_{1}}+\frac{d F_{k 2}}{d z_{2}}\right]_{L}+2 \operatorname{Re}\left[\frac{d F_{k 1}}{d z_{1}}+\frac{d F_{k 2}}{d z_{2}}\right]_{L}=\delta_{k 2}
\end{aligned}
$$

Como nestas expressões auxiliares os limites de integração não estão bem estabelecidos, uma melhor representação (ALBUQUERQUE, 2001) pode ser aplicada:

$$
\begin{aligned}
& 2 \operatorname{Re}\left[\left[\mu_{1} \frac{d F_{1}}{d z_{1}}+\mu_{2} \frac{d F_{2}}{d z_{2}}\right]\right]=-\delta_{k 1} \\
& 2 \operatorname{Re}\left[\left[\frac{d F_{1}}{d z_{1}}+\frac{d F_{2}}{d z_{2}}\right]\right]=\delta_{k 2}
\end{aligned}
$$

onde o colchete duplo representa que o valor primitivo está sendo avaliado no contorno fechado da integração. Esta integração, apesar de ser originalmente escrita no espaço real, foi transformada para o plano complexo, isto é, aparece agora como argumento do operador $R e$. O mapeamento no plano complexo é dado pela relação:

$$
Z^{\prime}=\left\{\begin{array}{l}
z_{1}^{\prime} \\
z_{2}^{\prime}
\end{array}\right\}=\left\{\begin{array}{l}
x_{1}^{\prime}+\mu_{1} x_{2}^{\prime} \\
x_{1}^{\prime}+\mu_{2} x_{2}^{\prime}
\end{array}\right\}
$$

A posição do ponto fonte neste mapeamento é denominada “ $Z$ ' ” e segue a expressão (A1.46). A solução das Equações (A1.44- A1.45) é dada por: 


$$
\begin{aligned}
& \frac{d F_{k 1}}{d z_{1}}=A_{k 1} \ln \left(z_{1}-z_{1}^{\prime}\right) \\
& \frac{d F_{k 2}}{d z_{2}}=A_{k 2} \ln \left(z_{2}-z_{2}^{\prime}\right)
\end{aligned}
$$

onde $A_{i k}$ são constantes complexas a serem determinadas como segue:

Considerando-se o ponto fonte na origem, ou seja, $\mathrm{z}^{\prime}=0$, tem - se:

$$
\begin{aligned}
& \frac{d F_{k 1}}{d z_{1}}=A_{k 1} \ln \left(z_{1}\right) \\
& \frac{d F_{k 2}}{d z_{2}}=A_{k 2} \ln \left(z_{2}\right)
\end{aligned}
$$

e substituindo-se estes valores nas expressões (A1.44- A1.45) tem-se:

$$
\begin{aligned}
& {\left[\left[2 \operatorname{Re}\left(\mu_{1} A_{k 1} \ln \left(z_{1}\right)+\mu_{2} A_{k 2} \ln \left(z_{2}\right)\right)\right]\right]=-\delta_{k 1}} \\
& {\left[\left[2 \operatorname{Re}\left(A_{k 1} \ln \left(z_{1}\right)+A_{k 2} \ln \left(z_{2}\right)\right)\right]\right]=\delta_{k 2}}
\end{aligned}
$$

A avaliação fechada de $\ln (z)$ é dada por (WYLIE \& BARRETT, 1995):

$$
[[\ln (z)]]=\oint_{L} \frac{1}{z_{k}} d z_{k}=2 \pi \cdot i
$$

Assim, as expressões (A1.51- A1.52) são facilmente avaliadas resultando em:

$$
2 \operatorname{Re}\left\{2 \cdot \pi \cdot i \cdot \mu_{1} A_{k 1}+2 \pi \cdot i \cdot \mu_{2} A_{k 2}\right\}=-\delta_{k 1}
$$

Recorrendo-se ainda a outras propriedades de funções complexas:

$$
\operatorname{Re}(z)=\frac{z+\bar{z}}{2}
$$

onde $\bar{z}$ é o conjugado de $z$, reescreve-se as Equações (A1.51) e (A1.52) como:

$$
\begin{aligned}
& A_{k 1}-\bar{A}_{k 1}+A_{k 2}-\bar{A}_{k 2}=\frac{\delta_{k 2}}{2 \pi i} \\
& \mu_{1} A_{k 1}-\bar{\mu}_{1} \bar{A}_{k 1}+\mu_{2} A_{k 2}-\bar{\mu}_{2} \bar{A}_{k 2}=\frac{-\delta_{k 1}}{2 \pi i}
\end{aligned}
$$

Têm-se duas equações (A1.56 e A1.57) para se resolver quatro constantes $A_{k 1}$, $A_{k 2}, \bar{A}_{k 1}$ e $\bar{A}_{k 2}$. Para completar o sistema, deve-se utilizar a equação de unicidade de deslocamentos (ALBUQUERQUE, 2001): 


$$
\oint_{\Gamma} u_{i} d \Gamma=0
$$

os deslocamentos $U_{k i}$ são determinados substituindo-se as Equações (A1.49- A1.50) em (A1.32) e (A1.33), isto é:

$$
\begin{aligned}
& U_{k 1}\left(z^{\prime}, z\right)=2 \operatorname{Re}\left[q_{11} A_{k 1} \ln \left(z_{1}-z_{1}^{\prime}\right)+q_{12} A_{k 2} \ln \left(z_{2}-z_{2}^{\prime}\right)\right] \\
& U_{k 2}\left(z^{\prime}, z\right)=2 \operatorname{Re}\left[q_{21} A_{k 1} \ln \left(z_{1}-z_{1}^{\prime}\right)+q_{22} A_{k 2} \ln \left(z_{2}-z_{2}^{\prime}\right)\right]
\end{aligned}
$$

com a matriz dos valores $q_{i j}(\mathrm{i}, \mathrm{j}=1,2)$ dada pela Equação (A1.31). Substituindo-se as Equações (A1.59) e (A1.60) em (A1.58) e lembrando-se da transformação da integral no plano real para o complexo e considerando-se $z^{\prime}=0$, escreve-se:

$$
\begin{aligned}
& 2 \operatorname{Re}\left[\left[q_{11} A_{k 1} \ln \left(z_{1}\right)+q_{12} A_{k 2} \ln \left(z_{2}\right)\right]\right]=0 \\
& \left.2 \operatorname{Re}\left[q_{21} A_{k 1} \ln \left(z_{1}\right)+q_{22} A_{k 2} \ln \left(z_{2}\right)\right]\right]=0
\end{aligned}
$$

e fazendo-se uso novamente das propriedades (A1.53 e A1.55), tem-se finalmente:

$$
\begin{aligned}
& q_{11} A_{k 1}-q_{11} \bar{A}_{k 1}+q_{12} A_{k 2}-q_{12} \bar{A}_{k 2}=0 \\
& q_{21} A_{k 1}-q_{21} \bar{A}_{k 1}+q_{22} A_{k 2}-q_{22} \bar{A}_{k 2}=0
\end{aligned}
$$

que juntamente com (A1.56- A1.57) formam o sistema complexo:

$$
\left[\begin{array}{cccc}
1 & -1 & 1 & -1 \\
\mu_{1} & -\bar{\mu}_{1} & \mu_{2} & -\bar{\mu}_{1} \\
q_{11} & -\bar{q}_{11} & q_{12} & -\bar{q}_{12} \\
q_{21} & -\bar{q}_{21} & q_{22} & -\bar{q}_{22}
\end{array}\right] \cdot\left\{\begin{array}{c}
A_{k 1} \\
\bar{A}_{k 1} \\
A_{k 2} \\
\bar{A}_{k 2}
\end{array}\right\}=\left\{\begin{array}{c}
\delta_{k 2} / 2 \pi \cdot i \\
-\delta_{k 1} / 2 \pi \cdot i \\
0 \\
0
\end{array}\right\}
$$

De onde determinam-se $A_{k i}$ que tornam as expressões (A1.49 e A1.50) em função de tensões, soluções de equilíbrio e da compatibilidade geométrica do problema bidimensional. Assim, os deslocamentos fundamentais (solução fundamental de deslocamento) são dados individualmente por:

$$
U_{k i}\left(z^{\prime}, z\right)=2 \operatorname{Re}\left[q_{i 1} A_{k 1} \ln \left(z_{1}-z_{1}^{\prime}\right)+q_{i 2} A_{k 2} \ln \left(z_{2}-z_{2}^{\prime}\right)\right]
$$


As forças de superfície fundamentais são obtidas substituindo-se as funções dadas pelas Equações (A1.49 - A1.50) nas expressões de tensões (A1.23 - A1.25) e usando-se também, a fórmula de Cauchy $\left(p_{j}=\sigma_{j k} \cdot \eta_{k}\right)$ :

$$
T_{k i}\left(z^{\prime}, z\right)=2 \operatorname{Re}\left[\begin{array}{c}
\frac{1}{\left(z_{1}-z_{1}^{\prime}\right)} g_{i 1}\left(\mu_{1} \eta_{1}-\eta_{2}\right) A_{k 1}+ \\
\frac{1}{\left(z_{2}-z_{2}^{\prime}\right)} g_{i 2}\left(\mu_{2} \eta_{1}-\eta_{2}\right) A_{k 2}
\end{array}\right]
$$

onde:

$$
\left[g_{i j}\right]=\left[\begin{array}{cc}
\mu_{1} & \mu_{2} \\
-1 & -1
\end{array}\right]
$$

e $\eta_{k}$ são as componentes do vetor normal externo. Para a determinação de tensões e de deformações é importante ainda apresentar as derivadas das soluções fundamentais.

Assim, para a solução fundamental de forças de superfície:

$$
T_{j i, k}=-2 R e\left[\begin{array}{c}
\frac{1}{\left(z_{1}-z_{1}^{\prime}\right)^{2}} R_{k 1} g_{i 1}\left(\mu_{1} \eta_{1}-\eta_{2}\right) A_{j 1}+ \\
\frac{1}{\left(z_{2}-z_{2}^{\prime}\right)^{2}} R_{k 2} g_{i 2}\left(\mu_{2} \eta_{1}-\eta_{2}\right) A_{j 2}
\end{array}\right]
$$

e para a solução fundamental de deslocamentos:

$$
U_{j i, k}=2 \operatorname{Re}\left[\frac{1}{\left(z_{1}-z_{1}^{\prime}\right)} R_{k 1} q_{i 1} A_{j 1}+\frac{1}{\left(z_{2}-z_{2}^{\prime}\right)} R_{k 2} q_{i 2} A_{j 2}\right]
$$

onde:

$$
\left[R_{k c}\right]=\left[\begin{array}{cc}
1 & 1 \\
\mu_{1} & \mu_{2}
\end{array}\right]
$$




\section{ANEXO 2 - ESTRATÉGIA NUMÉRICA PARA O CÁLCULO DO TERMO LIVRE - ANÁLISE DE PLASTICIDADE}

Neste Anexo é exposta a técnica desenvolvida para a determinação numérica do termo livre da solução fundamental anisotrópica de CRUSE \& SWEDLOW (1971), necessário para a determinação de deformações e tensões em pontos internos numa análise de plasticidade pelo MEC.

Como exposto no capítulo 5, na equação integral utilizada (Equação 5.28 ou Equação A2.1) para a análise em questão, existe um termo adicional $\left(g_{k l i j}\right)$ conhecido como termo livre, que é oriundo de derivações sobre integrais singulares e até então, sempre havia sido determinado de forma analítica, por exemplo, para a solução fundamental de Kelvin (BUI, 1978).

$$
\begin{aligned}
\varepsilon_{k l}(p)+\gamma \dot{\varepsilon}_{k l}(p)=\int_{\Gamma} \varepsilon_{k l i}^{*} p_{i} d \Gamma-\int_{\Gamma} p_{k l i}^{*} u_{i} d \Gamma-\gamma \int_{\Gamma} p_{k l i}^{*} \dot{u}_{i} d \Gamma+ \\
\quad+\int_{\Omega} \varepsilon_{k l i}^{*} b_{i} d \Omega+\int_{\Omega} t_{k l i j}^{*} \sigma_{i j}^{p} d \Omega+g_{k l i j} \sigma_{i j}^{p}(p)
\end{aligned}
$$

Porém, para meios anisotrópicos gerais, com a solução fundamental de CRUSE \& SWEDLOW (1971), dependente de variáveis complexas, a expressão do termo livre não é conhecida analiticamente. Nesta pesquisa, essa determinação é feita de forma numérica, maneira essa que constitui contribuição original ao MEC. 
Para tanto, visando-se o detalhamento desejado, pode-se partir da equação integral (A2.2):

$$
\begin{aligned}
u_{k}(p)+\dot{u}_{k}(p)=\int_{\Gamma} u_{k i}^{*} p_{i} d \Gamma-\int_{\Gamma} p_{k i}^{*} u_{i} d \Gamma-\gamma \int_{\Gamma} p_{k i}^{*} \dot{u}_{i} d \Gamma+ \\
\quad+\int_{\Omega} u_{k i}^{*} b_{i} d \Omega+\int_{\Omega} \varepsilon_{k i j}^{*} \sigma_{i j}^{p} d \Omega
\end{aligned}
$$

onde a segunda integral de domínio (Expressão A2.3) dá origem ao termo livre:

$$
I_{\varepsilon}=\int_{\Omega} \varepsilon_{k i j}^{*} \sigma_{i j}^{p} d \Omega
$$

após a diferenciação da expressão (A2.3) em relação à posição do ponto fonte. Para o desenvolvimento dessa derivação é necessário a utilização de um sistema de coordenadas polares cujo centro está localizado no ponto fonte "s", sendo também a origem de uma sub-região $\Omega_{\mathrm{e}}$ de raio $\varepsilon$, extraída do corpo, tão pequena quanto se queira (Figura A2.1):

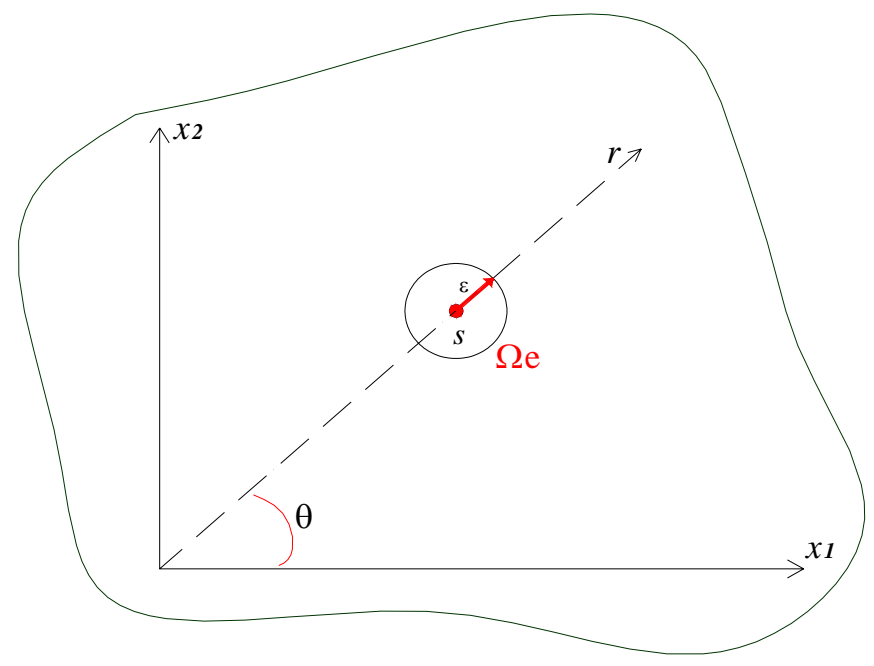

FIGURA A2.1 - Sub-região $\Omega_{\mathrm{e}}$ de raio $\varepsilon$, extraída do corpo.

de maneira que seja possível escrever a Equação (A2.3) como:

$$
I_{\varepsilon}=\lim _{\varepsilon \rightarrow 0} \frac{\partial}{\partial x_{l}} \int_{\Omega-\Omega_{e}} \varepsilon_{k i j}^{*}(s, p) \sigma_{i j}^{p} d \Omega
$$

Para o desenvolvimento do limite (A2.4), a equação deve ser escrita em coordenadas polares: 


$$
I_{\varepsilon}=\int_{0}^{2 \pi}\left[\lim _{\varepsilon \rightarrow 0} \frac{\partial}{\partial x_{l}} \int_{\varepsilon}^{r} \varepsilon_{k i j}^{*}(\theta, r(s, p)) \cdot \sigma_{i j}^{p} d r\right] d \theta
$$

E, após alguns desenvolvimentos, obtém-se (CODA, 2000):

$$
I_{\varepsilon}=\int_{0}^{2 \pi} \lim _{\varepsilon \rightarrow 0}\left[\int_{\varepsilon}^{r} \frac{\partial \varepsilon_{k i j}^{*}(\theta, r)}{\partial x_{l}(s)} \cdot \sigma_{i j}^{p} \cdot r d r-\varepsilon_{k i j}^{*}(\theta, \varepsilon) \cdot \sigma_{i j}^{p} \cdot \varepsilon \cdot r,_{l}\right] d \theta
$$

que, considerando-se a expressão para a primeira derivada da solução fundamental de deslocamentos (Equação A1.70) escrita em coordenadas polares:

$$
U_{j i, k}^{*}=\frac{2 \operatorname{Re}}{r}\left[\frac{R_{k 1} q_{i 1} A_{j 1}}{\cos (\theta)+\mu_{1} \operatorname{sen}(\theta)}+\frac{R_{k 2} q_{i 2} A_{j 2}}{\cos (\theta)+\mu_{2} \operatorname{sen}(\theta)}\right]
$$

e que:

$$
U_{j i, k}^{*}=\varepsilon_{k i j}^{*}(\theta, r)=\frac{1}{r} \psi_{k i j}(\theta)
$$

A Equação (A2.6) pode ser escrita como:

$$
I_{\varepsilon}=\int_{0}^{2 \pi} \lim _{\varepsilon \rightarrow 0}\left[\int_{\varepsilon}^{r} \frac{\partial \varepsilon_{k i j}^{*}(\theta, r)}{\partial x_{l}(s)} \cdot \sigma_{i j}^{p} \cdot r d r\right] d \theta-\int_{0}^{2 \pi} \psi_{k i j}(\theta) \cdot r_{l} d \theta \cdot \sigma_{i j}^{p}
$$

onde aparecem dois termos, no segundo membro da equação, sendo o primeiro resolvido no código com aproximação de células de domínio e o segundo, responsável pela origem do termo livre. Esse termo é o determinado numericamente na pesquisa.

Assim, escrevendo-o separadamente, tem-se:

$$
g_{j i k l}=\int_{0}^{2 \pi} \psi_{k i j}(\theta) \cdot r, d \theta=\int_{0}^{2 \pi} \varepsilon_{k i j}^{*}(\theta) \cdot r \cdot r,,_{l} \cdot d \theta
$$

ou ainda, utilizando-se (A2.7):

$$
g_{j i k l}=2 \operatorname{Re} \int_{0}^{2 \pi}\left[\frac{R_{k 1} q_{i 1} A_{j 1}}{\cos (\theta)+\mu_{1} \operatorname{sen}(\theta)}+\frac{R_{k 2} q_{i 2} A_{j 2}}{\cos (\theta)+\mu_{2} \operatorname{sen}(\theta)}\right] \cdot r,_{l} \cdot d \theta
$$


Expressão essa que foi calculada utilizando integração numérica com pontos de Gauss. Esquematicamente, tem-se:

$$
g_{j i k l}=\int_{0}^{2 \pi} f_{j i k l}(\theta) d \theta=\pi \int_{-1}^{1} f_{j i k l}\left(\theta_{i}\right) d \xi=\pi \cdot \sum_{i=1}^{N G} f_{j i k l}\left(\theta_{i}\right) \omega_{i}
$$

onde:

$$
\theta_{i}=\theta_{1} \phi_{1}+\theta_{2} \phi_{2}=2 \pi \cdot \phi_{2} ; \quad \phi_{1}=\frac{1-\xi}{2} ; \quad \phi_{2}=\frac{1+\xi}{2}
$$

Na Equação (A2.11), $r_{{ }_{l}}$ são cossenos diretores. Assim, dependendo-se da direção de derivada em relação ao ponto fonte, tem-se para $f_{j i k l}(\theta)$ :

$$
\begin{aligned}
& f_{j i k 1}(\theta)=\left[\frac{R_{k 1} q_{i 1} A_{j 1}}{\cos (\theta)+\mu_{1} \operatorname{sen}(\theta)}+\frac{R_{k 2} q_{i 2} A_{j 2}}{\cos (\theta)+\mu_{2} \operatorname{sen}(\theta)}\right] \cdot \cos (\theta) \\
& f_{j i k 2}(\theta)=\left[\frac{R_{k 1} q_{i 1} A_{j 1}}{\cos (\theta)+\mu_{1} \operatorname{sen}(\theta)}+\frac{R_{k 2} q_{i 2} A_{j 2}}{\cos (\theta)+\mu_{2} \operatorname{sen}(\theta)}\right] \cdot \operatorname{sen}(\theta)
\end{aligned}
$$

Podendo-se então realizar as integrações numéricas necessárias. Ao todo são doze integrações, encontrando-se doze termos livres que formam a matriz 3 x 3 necessária para a análise de plasticidade. Essa matriz possui a forma:

$$
\left[g_{j i k l}\right]_{3 \times 3}=\left[\begin{array}{ccc}
g_{1111} & g_{1221} & g_{1121} \\
g_{2112} & g_{2222} & g_{2122} \\
\frac{\left(g_{1112}+g_{2111}\right)}{2} & \frac{\left(g_{1222}+g_{2221}\right)}{2} & \frac{\left(g_{1122}+g_{2121}\right)}{2}
\end{array}\right]
$$

Para finalizar este Anexo 2, é importante ainda dizer que não é preciso muitos pontos de Gauss para se ter uma boa integração das expressões apresentadas, sendo usados no trabalho, 10 pontos de Gauss. 


\section{ANEXO 3 - DETERMINAÇão dAS COORDENADAS ADMENSIONAIS DE UM PONTO CARTESIANO}

No cálculo de funções de forma presentes, por exemplo, na matriz (10.12) do capítulo 10, é preciso se conhecer as coordenadas adimensionais do nó a que as funções se referem. Geralmente na geração de malha, ou de nós de fibras, se conhece apenas as coordenadas cartesianas dos nós. Assim, descreve-se neste Anexo 3 a metodologia empregada para o cálculo de coordenadas adimensionais necessárias para a determinação das funções de forma empregadas no trabalho, quando se conhecia apenas as coordenadas cartesianas dos pontos. Salienta-se que este procedimento também pode ser encontrado em CODA (1990).

Para um ponto “ $q$ ” inserido num elemento finito, como se mostra na Figura A3.1, pode-se escrever explicitamente :

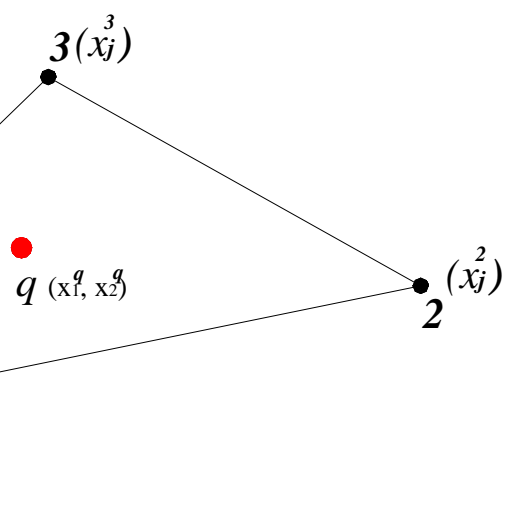

FIGURA A3.1 - Um ponto “ $q$ ” inserido numa posição qualquer de um elemento finito.

$$
x_{j}^{q}=\xi_{1}^{q} x_{j}^{1}+\xi_{2}^{q} x_{j}^{2}+\xi_{3}^{q} x_{j}^{3} \quad(j=1,2)
$$


Podendo-se calcular as variáveis adimensionais $\xi_{\alpha}^{q}$ para o ponto “ $q$ ” em função do sistema de coordenadas cartesiano $\left(x_{1}, x_{2}\right)$ por meio da Equação (A3.2):

$$
\xi_{\alpha}^{q}=\frac{1}{2 A}\left(2 A_{0}^{\alpha}+b^{\alpha} x_{1}^{q}+a^{\alpha} x_{2}^{q}\right)
$$

onde:

$$
\begin{aligned}
& a^{\alpha}=x_{1}^{k}-x_{1}^{m} \\
& b^{\alpha}=x_{2}^{m}-x_{2}^{k} \\
& 2 A_{0}^{\alpha}=x_{1}^{m} x_{2}^{k}-x_{1}^{k} x_{2}^{m} \\
& A=\frac{1}{2}\left(b^{1} a^{2}-b^{2} a^{1}\right)
\end{aligned}
$$

e as variáveis $\alpha, m$ e $k$ respeitam a relação cíclica:

$$
\alpha=1,2,3 ; \quad m=2,3,1 ; \quad k=3,1,2
$$

por exemplo, se $\alpha=1, m=2$ e $k=3$.

Especificamente no capítulo 10, quando da necessidade de se saber se um ponto de fibra estava inserido ou não num determinado elemento finito, calcula-se para o nó de fibra em questão, num loop sobre o número de elementos finitos, suas três coordenadas adimensionais por meio da Equação (A3.2). Quando ocorrer dos três valores serem maiores ou iguais a zero, significa que o nó de fibra pertence ao elemento finito cujas coordenadas cartesianas dos vértices $\left(x_{j}^{\mathrm{j}}\right)$ estão sendo usadas para a determinação das coordenadas adimensionais do nó de fibra em questão. 